\title{
29. DIATOM BIOSTRATIGRAPHY: KERGUELEN PLATEAU AND PRYDZ BAY REGIONS OF THE SOUTHERN OCEAN ${ }^{1}$
}

\author{
Jack G. Baldauf ${ }^{2}$ and John A. Barron ${ }^{3}$
}

\begin{abstract}
Samples were examined for diatoms from 22 holes at 11 sites cored by ODP Leg 119 on the Kerguelen Plateau and in Prydz Bay, East Antarctica. Diatoms were observed in Oligocene through Holocene sediments recovered from the Kerguelen Plateau. The diatom flora from the Kerguelen Plateau is characterized by species such as Azpeitia oligocenica, Rocella gelida, Rocella vigilans, and Synedra jouseana in the Oligocene and Crucidenticula nicobarica, Denticulopsis hustedtii, Nitzschia miocenica, and Thalassiosira miocenica in the Miocene. This somewhat cosmopolitan assemblage gives way to a Pliocene and Holocene assemblage characterized by species such as Nitzschia kerguelensis, Thalassiosira inura, and Thalassiosira torokina, which are endemic to the Southern Ocean region. Samples examined from Prydz Bay are generally devoid of diatoms. The exception is Site 739, where diatoms occur sporadically in lower Oligocene and upper Miocene through Quaternary sediments.

The Leg 119 diatom biostratigraphic results allow the development of a stratigraphic framework for the Indian sector of the Southern Ocean. This diatom zonation integrates diatom zonations developed previously for other sectors of the Southern Ocean. The zonation proposed here is based on biostratigraphic events of both geographically widespread and endemic species calibrated to the paleomagnetic stratigraphy. As such, this zonation has application throughout the Southern Ocean and allows correlation from the southern high latitudes to the low latitudes.
\end{abstract}

\section{INTRODUCTION}

Ocean Drilling Program (ODP) Leg 119 cored 22 holes at 11 sites in the Southern Ocean on the Kerguelen Plateau (Sites 736-738 and 744-746) and in Prydz Bay, East Antarctica (Sites 739-743) (Table 1 and Fig. 1). The scientific objectives of this cruise differed for each region. The paleoceanographic objectives on the Kerguelen Plateau were (1) to recover an expanded sedimentary sequence consisting of Neogene calcareous and biosiliceous oozes in order to document the development and history of the Antarctic Polar Frontal Zone, (2) to determine the evolution of Antarctic water masses and their response to climatic variation, and (3) to document the tectonic and sedimentation history on the Kerguelen Plateau.

Paleoceanographic/paleoclimatic objectives at the Prydz Bay sites were (1) to determine the glacial history of East Antarctica, (2) to document the development from a preglacial to glacial continental climate on East Antarctica, and (3) to document the development of the continental shelf environment (depth, temperature, and sea ice cover) in order to assess the response of this region to climatic change.

An additional objective for both regions was to develop an integrated siliceous and calcareous biostratigraphy that is calibrated to magnetostratigraphy. Such results would provide a biostratigraphic reference section for the southern high latitudes that would allow testing the synchroneity of specific biostratigraphic events, as well as allow incorporation of the Southern Ocean sequences into an overall global chronostratigraphic framework.

This manuscript presents the diatom biostratigraphic results for ODP Leg 119. As discussed in the following sections, numerous diatom zonations (Fig, 2) have been established previously

\footnotetext{
${ }^{1}$ Barron, J., Larsen, B., et al., 1991. Proc. ODP, Sci. Results, 119: College Station, TX (Ocean Drilling Program).

${ }^{2}$ Department of Geological Oceanography, Texas A\&M University, College Station, TX 77843, U.S.A., and Ocean Drilling Program, Texas A\&M University, 1000 Discovery Drive, College Station, TX 77845-9547, U.S.A.

${ }^{3}$ Paleontology and Stratigraphy Branch, U.S. Geological Survey, 345 Middlefield Road, Menlo Park, CA 94025, U.S.A.
}

Table 1. Geographic location and water depth of holes cored during Leg 119.

\begin{tabular}{lccr}
\hline & & & \\
Hole & $\begin{array}{c}\text { Latitude } \\
\left({ }^{\circ} \mathrm{S}\right)\end{array}$ & $\begin{array}{c}\text { Wongitude } \\
\left({ }^{\circ} \mathrm{E}\right)\end{array}$ & $\begin{array}{c}\text { Water } \\
\text { depth } \\
(\mathrm{m})\end{array}$ \\
\hline 736A & 49.40 & 71.66 & 629.0 \\
$736 \mathrm{~B}$ & 49.40 & 71.66 & 628.1 \\
$736 \mathrm{C}$ & 49.40 & 71.66 & 629.0 \\
$737 \mathrm{~A}$ & 50.23 & 73.03 & 564.0 \\
$737 \mathrm{~B}$ & 50.23 & 73.03 & 564.0 \\
$738 \mathrm{~A}$ & 62.71 & 82.78 & 2252.7 \\
$738 \mathrm{~B}$ & 62.71 & 82.79 & 2252.5 \\
$738 \mathrm{C}$ & 62.71 & 82.79 & 2252.5 \\
$739 \mathrm{~A}$ & 67.28 & 71.77 & 412.4 \\
$739 \mathrm{~B}$ & 67.28 & 71.77 & 412.4 \\
$739 \mathrm{C}$ & 67.28 & 71.77 & 412.4 \\
$740 \mathrm{~A}$ & 68.76 & 76.68 & 807.5 \\
$740 \mathrm{~B}$ & 68.76 & 76.68 & 814.4 \\
$741 \mathrm{~A}$ & 68.38 & 76.38 & 551.4 \\
$742 \mathrm{~A}$ & 67.55 & 75.41 & 415.7 \\
$743 \mathrm{~A}$ & 66.92 & 74.69 & 988.7 \\
$744 \mathrm{~A}$ & 61.58 & 80.59 & 2307.2 \\
$744 \mathrm{~B}$ & 61.56 & 80.59 & 2306.5 \\
$744 \mathrm{C}$ & 61.58 & 80.59 & 2308.0 \\
$745 \mathrm{~A}$ & 59.59 & 85.85 & 4093.0 \\
$745 \mathrm{~B}$ & 59.59 & 85.85 & 4082.5 \\
$746 \mathrm{~A}$ & 59.57 & 85.87 & 4059.5 \\
\hline & & &
\end{tabular}

for the Southern Ocean. Results from Sites 736 through 746 provide the means to evaluate these zonations and develop a zonal scheme with broad geographic application, without sacrificing the stratigraphic resolution. The new zonation presented here follows previous zonations by making use of both species that have wide geographic distribution and endemic marker species to recognize individual zones.

\section{METHODS}

Approximately $1.5 \mathrm{~cm}^{3}$ of sample was placed into a $400-\mathrm{mL}$ beaker and disaggregated by the addition of $10 \mathrm{~mL}$ of $30 \%$ 


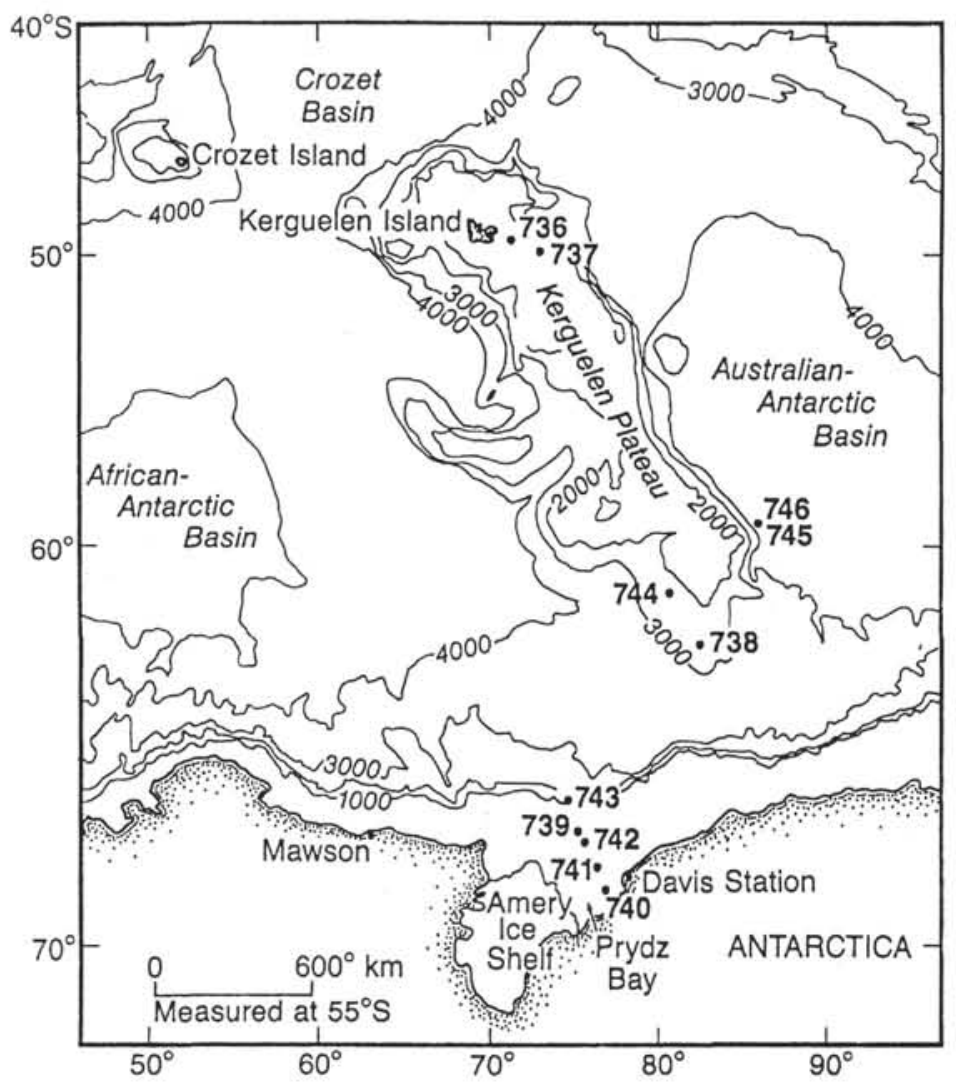

Figure 1. The location of Leg 119 Sites 736 through 746 is divided into two geographic regions: Kerguelen Plateau, Southern Ocean (Sites 736-738 and 744-746) and Prydz Bay, East Antarctica (Sites 739-743). Bathymetry is in meters.

hydrogen peroxide. Then $30 \mathrm{~mL}$ of $37 \% \mathrm{HCl}$ was added and the sample was gently heated until carbonates and organic carbons were removed. The sample was neutralized by the addition of approximately $300 \mathrm{~mL}$ of distilled water and decanted after $2 \mathrm{hr}$ of settling. The decanting process was repeated until a $\mathrm{pH}$ of approximately 7 was achieved. Strewn slides were prepared on 22 $\times 30 \mathrm{~mm}$ (No. 1 thickness) cover glass and mounted in Hyrax on $22 \times 75 \mu \mathrm{m}$ glass slides. Shipboard samples from critical stratigraphic intervals were reprocessed following the cruise to verify shipboard results.

Strewn slides of acid-cleaned material prepared aboard JOIDES Resolution were reexamined often, together with additional samples processed at shore-based facilities. One strewn slide was examined in its entirety at $\sim \times 800$ with species identifications confirmed at $\times 1250$. Species were recorded as abundant (A) if two or more specimens were present in one field of view at $\sim \times 800$, common (C) if one specimen occurred in two fields of view, few (F) if one specimen was observed in each horizontal transect, and rare $(R)$ if specimens were encountered less commonly than that. The quality of fossil preservation was recorded subjectively, based on valve morphology (occurrence of chemical pitting or etching) and the number of finely silicified specimens compared to the number of heavily silicified forms.

\section{ZONATION}

Numerous Cenozoic diatom zonations (Fig. 2) have been established for the Southern Oceans (McCollum, 1975; Schrader, 1976; Weaver and Gombos, 1981; Ciesielski, 1983; Gombos, 1976; Fenner, 1984, 1985; Gombos and Ciesielski, 1983) or are currently under development (Gersonde and Burckle, 1990; Bal- dauf and Barron, herein). The evolution of the Southern Ocean diatom zonation from that first defined by McCollum (1975; Deep Sea Drilling Project [DSDP] Leg 28) to the zonation presented in this manuscript reflects an enrichment in the scientific data base (i.e., the number of sites and cores, core quality, and paleomagnetic sequences for calibration) available from the Southern Ocean.

The pioneering work of McCollum (1975) provided the biostratigraphic framework used at least in part by the subsequent diatom studies. McCollum's zonation consists of 13 middle Miocene to Holocene zones. The Miocene portion of this zonation is based generally on the stratigraphic ranges of cosmopolitan species such as Coscinodiscus lewisianus, Denticulopsis hustedtii, and Denticulopsis lauta; the Pliocene to Holocene portion is based generally on the stratigraphic ranges of species endemic to the Southern Ocean such as Nitzschia praeinterfrigidaria, Nitzschia interfrigidaria, and Thalassiosira kolbei.

McCollum's (1975) zonation is applicable to and provides a regional stratigraphic framework for the Southern Ocean. However, the more recent studies reviewed here have noted several difficulties inherent in this zonation: (1) numerous zones are defined on the last occurrence rather than the first occurrence of specific species, which makes it difficult to distinguish between a true last occurrence of a species and that of a reworked specimen; (2) calibration of specific stratigraphic events to magnetostratigraphic records is undocumented; and (3) zonal names are typically complex, for example, the Rhizosolenia barboi/Nitzschia kerguelensis Zone of McCollum implies a concurrent-range zone based on the joint occurrence of $R$. barboi and $N$. kerguelensis; however, the zone has a base defined by the last 


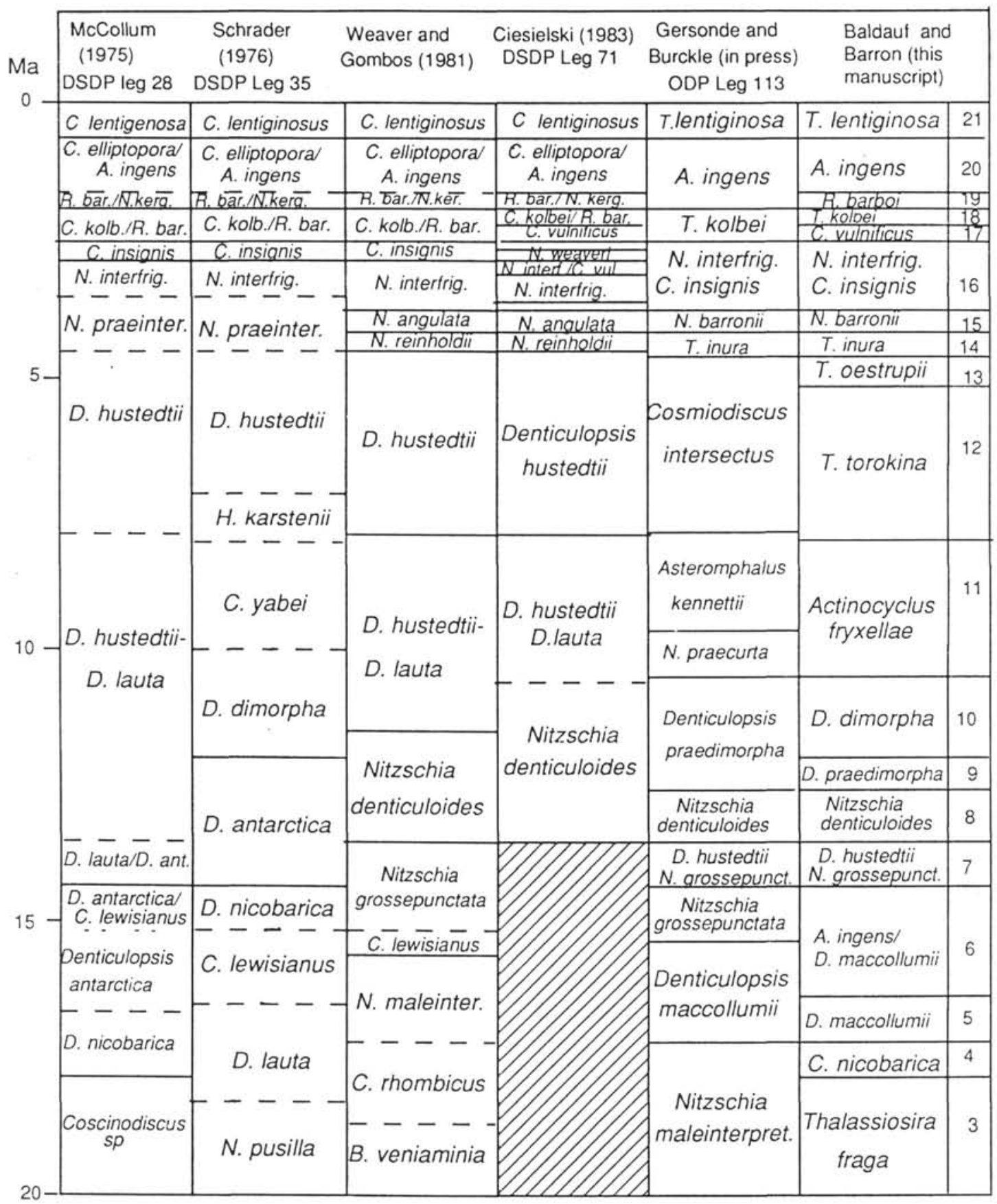

Figure 2. Correlation of the Quaternary to Oligocene diatom zonation proposed in this manuscript with those previously proposed for the Southern Ocean. Dashed lines represent uncertainties in correlation.

occurrence of $T$. kolbei and a top defined by the last occurrence of $R$, barboi.

Schrader (1976) utilized the Pliocene to Holocene portion of McCollum's (1975) zonation, but defined an alternate zonal scheme for the Oligocene through Miocene interval. Schrader based the Oligocene through Miocene portion of his zonation generally on the stratigraphic ranges of species such as Coscinodiscus yabei (currently referred to as Thalassiosira yabei), Denticulopsis nicobarica (currently referred to as Crucidenticula nicobarica), Coscinodiscus lewisianus, Thalassiosira spumellaroides, Thalassiosira spinosa, and Nitzschia maleinterpretaria. Unfortunately, the study by Schrader lacked independent paleo- magnetic data to allow testing the synchroneity of individual events.

Weaver and Gombos (1981) further advanced Southern Ocean diatom biostratigraphy by incorporating portions of McCollum's (1975) and Schrader's (1976) zonations into a new Neogene zonation. The upper Pliocene to Holocene portion of Weaver and Gombos's zonation somewhat follows that of McCollum and the Miocene portion generally follows the zonal scheme of Schrader. However, improvements to these previous zonations were made by replacing the interval representing the Nitzschia praeinterfrigidaria Zone of McCollum (1975) with the Nitzschia reinholdii, Nitzschia angulata, and Nitzschia interfrigidaria Zones. In addi- 


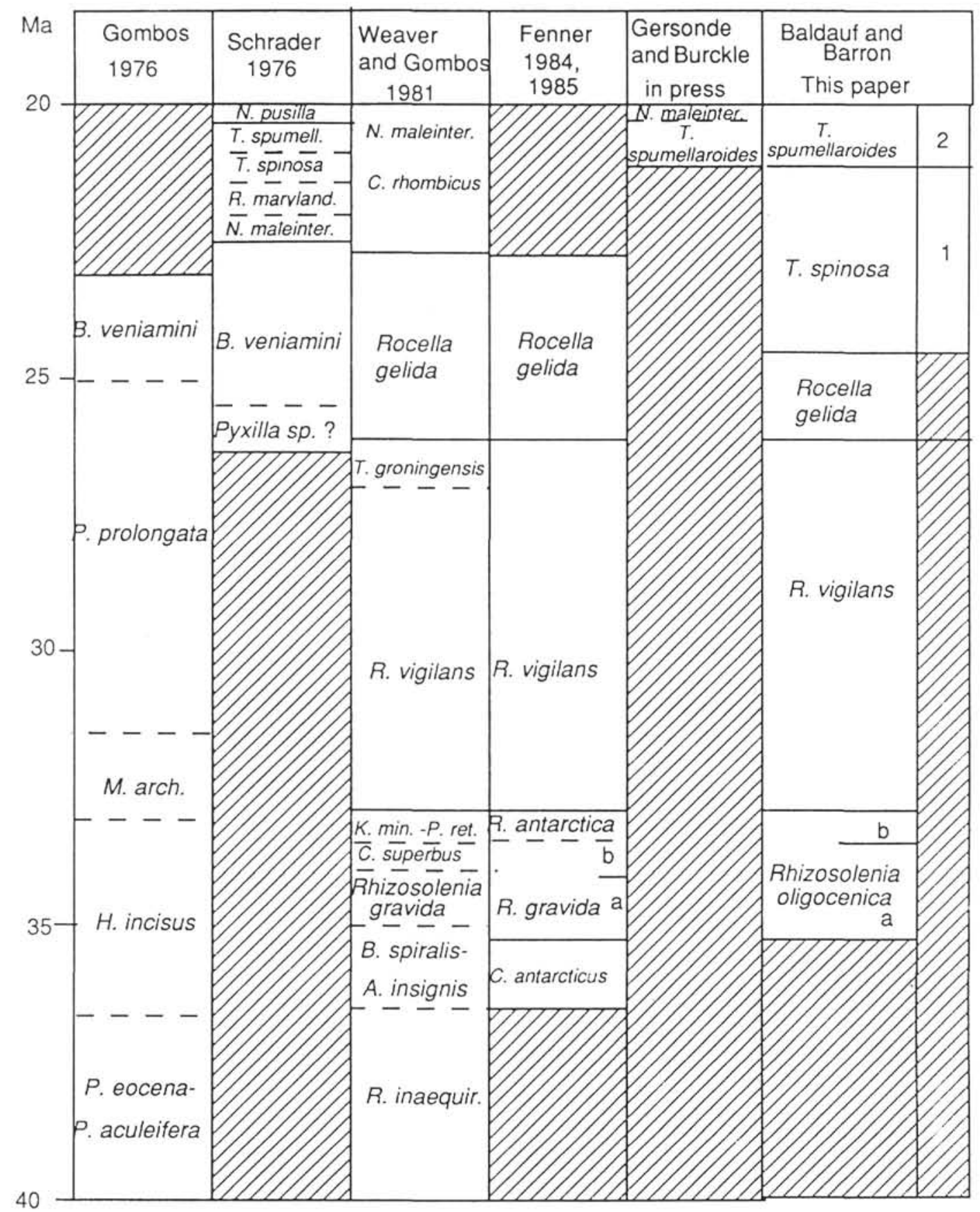

Figure 2 (Continued).

tion, Weaver and Gombos defined the Nitzschia grossepunctata, Nitzschia denticuloides, and Coscinodiscus rhombicus Zones (see Fig. 2) for use in the Miocene.

Ciesielski $(1983,1985)$ improved the stratigraphic usefulness of Neogene diatoms in the Southern Ocean by further revising the previously defined late Miocene to Holocene zones (those of McCollum, 1975; Weaver and Gombos, 1981). In doing so, Ciesielski (1983) defined five new Pliocene to Holocene zones to increase age resolution and redefined portions of most of the remaining zones. In addition, Ciesielski (1983) published the calibration of diatom biostratigraphic events to paleomagnetic stratigraphy. Reliable calibration, however, is limited to the Pliocene and younger interval.
More recent diatom studies (Gersonde and Burckle, 1990; Baldauf and Barron, herein) based on ODP sediments from the Southern Ocean provide the opportunity to evaluate the usefulness of these previously established zones and to calibrate specific events to magnetostratigraphy and the geopolarity time scale. In addition, the recent studies allow integration of biostratigraphic events based on siliceous and calcareous microfossils.

Gersonde and Burckle (1990) recognized the numerous difficulties inherent in the previously defined Neogene diatom zonations. In addition, Gersonde and Burckle pointed out the need to refine the stratigraphic resolution by decreasing the duration of several zones (see Fig. 2). Following Schrader (1976) and Fenner $(1984,1985)$ Gersonde and Burckle noted that deep-sea cores in 
the Southern Ocean commonly contain reworked older diatoms. Consequently, use of last-occurrence datums to define zonal boundaries in this region requires caution (Gersonde and Burckle, 1990). As a result, Gersonde and Burckle redefined the previously proposed Neogene diatom zonations by incorporating previously well-established and easily recognizable zones with new zones proposed for the problem intervals.

Although one recognizes the potential difficulty in using the last occurrence of a species as a stratigraphic horizon, it should be noted that the comparison of results from Ciesielski (1983), Gersonde and Burckle (1990), and this study allows testing the synchroneity of events in the Southern Ocean. Such an evaluation indicates discrepancies in the chronostratigraphic placement of specific events and dictates partial revision of the previously defined zonations (see Fig. 2), but such an evaluation also supports the stratigraphic usefulness of several stratigraphic events based on last occurrences.

In addition to the preceding Neogene zonations, several authors (Gombos, 1976; Weaver and Gombos, 1981; Fenner, $1984,1985)$ have proposed diatom zonations for the Oligocene (Fig. 2). Gombos (1976) proposed five diatom zones spanning the late Eocene to early Miocene based on results from DSDP Site 328 in the Falkland Plateau region. Although these zones proved of local stratigraphic use, their usefulness was limited in part by the lack of age calibration or correlation of these zones to other regions.

Gombos and Ciesielski (1983) abandoned the previously defined zonation of Gombos (1976) and defined an Oligocene to Miocene diatom zonation for use in the Southern Ocean. However, the utility of the zonation is limited because specific stratigraphic events were not calibrated to an independent time control. As a result, the age calibration and the chronostratigraphic placement of these zones differs among workers (for discussion see Baldauf and Monjanel, 1989). Fenner $(1984,1985)$ proposed the most recent Oligocene diatom zonation for use in the Southern Ocean (Fig. 2). This zonation subdivides the Oligocene into five zones based primarily on cosmopolitan species (for discussion see Baldauf and Monjanel, 1989).

Leg 119 provides an additional opportunity to examine the usefulness of the previously proposed Southern Ocean diatom zonations. Emphasis during this study was placed on the ability to recognize specific zones not only in the Kerguelen Plateau region, but also throughout the Southern Ocean. The stratigraphic sequences cored during Leg 119 contain a diverse flora composition and a recognizable paleomagnetic stratigraphy that, in conjunction with previous efforts, provide an overall stratigraphic framework applicable throughout the Southern Ocean.

Throughout the following discussion of the Leg 119 diatom zones, the reader is referred to Table 2 and Figures 2 and 3, which compare the Leg 119 diatom zonation with previously published Southern Ocean diatom zonations and which show the diatom events marking the zonal boundaries used in this manuscript. We use the notation for geomagnetic polarity chrons proposed by Tauxe et al. (1984) and that for subchrons proposed in the "Explanatory Notes" section of Barker, Kennett, et al. (1988, p. 24-25). Ages are from the Berggren et al. (1985) geomagnetic polarity time scale.

\section{Diatom Zonation}

Table 2 shows specific diatom events and the age calibration previously and currently defined for the Southern Ocean region. Table 3 shows the primary and secondary datums related to Neogene Southern Ocean diatom (NSOD) zones employed here as well as the occurrence of those datums and other stratigraphically useful datums recognized in the Leg 119 holes.

\section{NSOD Zone 21 (Thalassiosira lentiginosa Partial-Range Zone)}

Top. Present.

Base. Last occurrence of Actinocyclus ingens.

Author. McCollum (1975); renamed informally by Kellogg and Kellogg (1986), formally renamed by Gersonde and Burckle (1990).

Age. Present-0.62 Ma. The last occurrence of A. ingens is calibrated to the lowermost portion of the Brunhes normal polarity event (Subchron C1N-1; Ciesielski, 1983; Gersonde and Burckle, 1990; Baldauf and Barron, herein).

\section{NSOD Zone 20 (Actinocyclus ingens Partial-Range Zone)}

Top. Last occurrence of Actinocyclus ingens.

Base. Last occurrence of Rhizosolenia barboi.

Author. Ciesielski (1983); renamed by Gersonde and Burckle (1990).

Age. 0.62-1.58 Ma. Ciesielski (1983) calibrated the last occurrence of $R$. barboi with the upper portion of the Matuyama Chronozone (Subchron C1R-2).

Discussion. Although the occurrence of $R$. barboi is somewhat sporadic in the Pliocene sediments at Site 736 , this species has a consistent occurrence at Site 745 . As a result, the authors adhere to the original definition of this zone. Gersonde and Burckle (1990) emended the base of this zone by incorporating the interval representing the underlying Rhizosolenia barboi/Nitzschia kerguelensis Zone (McCollum, 1975) and defining the base of this modified zone at the last occurrence of Thalassiosira kolbei. NSOD Zone 20 as used here correlates with the upper two-thirds of the Actinocyclus ingens Zone as defined by Gersonde and Burckle (1990) and is equivalent to the Coscinodiscus elliptopora/Actinocyclus ingens Zone of Ciesielski (1983).

\section{NSOD Zone 19 (Rhizosolenia barboi Partial-Range Zone)}

Top. Last occurrence of Rhizosolenia barboi.

Base. Last occurrence of Thalassiosira kolbei.

Author. McCollum (1975), modified by Ciesielski (1983); renamed by Baldauf and Barron (herein).

Age. 1.58-1.89 Ma. The last occurrence of $T$. kolbei correlates with Subchron C2N-1 (Ciesielski, 1983; Gersonde and Burckle, 1990).

Discussion. Ciesielski (1983) emended the Rhizosolenia barboi/Nitzschia kerguelensis Zone of McCollum (1975) to be the interval between the last occurrence of $R$. barboi and the last occurrence of Coscinodiscus kolbei (now referred to as Thalassiosira kolbei). This zone is renamed NSOD Zone 19 (the Rhizosolenia barboi Zone) because $N$. kerguelensis is no longer part of the zonal definition. This zone correlates with the lower portion of the A. ingens Zone as defined by Gersonde and Burckle (1990) and with the Rhizosolenia barboi/Nitzschia kerguelensis Zone of Ciesielski (1983).

\section{NSOD Zone 18 (Thalassiosira kolbei Partial-Range Zone)}

Top. Last occurrence of Thalassiosira kolbei.

Base. Last occurrence of Coscinodiscus vulnificus.

Author. Ciesielski (1983); renamed by Baldauf and Barron (herein).

Age. 1.89-2.22 Ma. Weaver and Gombos (1981) correlated the last occurrence of $C$. vulnificus with Subchron C2R-1.

Discussion. We adhere to the original definition of this zone as defined by Ciesielski (1983) based on the reliability of defining the event in the Kerguelen Plateau and Weddell Sea regions of the Southern Ocean. To adhere to the recent taxonomic transfer of Coscinodiscus kolbei to Thalassiosira by Gersonde (1990), Gersonde and Burckle (1990) redefined this zone. Although Gersonde 
Table 2. Specific diatom events and the age calibrations previously and currently defined for the Southern Ocean region.

\begin{tabular}{|c|c|c|c|}
\hline \multirow[b]{2}{*}{ Datum } & \multirow{2}{*}{$\begin{array}{c}\text { Reference } \\
\text { for zonal/ } \\
\text { subzonal } \\
\text { marker } \\
\text { event }^{\mathrm{a}}\end{array}$} & \multicolumn{2}{|l|}{ Age $^{\mathrm{b}}$ (calibration) } \\
\hline & & (Ma) & Reference $^{\mathrm{c}}$ \\
\hline LCO Hemidiscus karstenii & & $0.195(\mathrm{C} 1 \mathrm{~N}-1)$ & 1 \\
\hline \multirow[t]{2}{*}{ LO Actinocyclus ingens } & $\mathrm{A}, \mathrm{B}, \mathrm{C}$ & $0.6(\mathrm{C} 1 \mathrm{~N}-1)$ & 3 \\
\hline & & $0.62(\mathrm{C} 1 \mathrm{~N}-1)$ & 2 \\
\hline LO Coscinodiscus elliptopora & & $0.65(\mathrm{C} 1 \mathrm{~N}-1)$ & 2 \\
\hline LO Rhizosolenia barboi & $A, C$ & $1.58(\mathrm{C} 1 \mathrm{R}-2)$ & 2 \\
\hline \multirow[t]{2}{*}{ LO Thalassiosira kolbei } & $\mathrm{A}, \mathrm{B}, \mathrm{C}$ & $1.89(\mathrm{C} 2 \mathrm{~N}-1)$ & 2 \\
\hline & & $-1.8(\mathrm{C} 2 \mathrm{~N}-1)$ & 3 \\
\hline FO C. elliptopora & & $2.2(-)$ & 2 \\
\hline \multirow{2}{*}{ LO Coscinodiscus vulnificus } & $\mathrm{A}, \mathrm{C}$ & $2.22(\mathrm{C} 2 \mathrm{R}-1)$ & 4 \\
\hline & & $2.2-2.3(\mathrm{C} 2 \mathrm{R}-1)$ & 10 \\
\hline \multirow[t]{3}{*}{ LO Cosmiodiscus insignis } & $\mathrm{A}, \mathrm{B}, \mathrm{C}$ & $2.49(\mathrm{C} 2 \mathrm{AN}-1)$ & 2,5 \\
\hline & & $2.5(\mathrm{C} 2 \mathrm{AN}-1)$ & 3 \\
\hline & & $2.4-2.45(\mathrm{C} 2 \mathrm{R}-1)$ & 10 \\
\hline \multirow[t]{2}{*}{ LO Nitzschia weaveri } & A & $2.64(-)$ & 2 \\
\hline & & $1.75-1.9(\mathrm{C} 2 \mathrm{~N}-1)$ & 10 \\
\hline \multirow[t]{2}{*}{ FO Nitzschia kerguelensis } & & $2.7(\mathrm{C} 2 \mathrm{AN}-1)$ & 5 \\
\hline & & $1.9-2.0(\mathrm{C} 2 \mathrm{R}-1)$ & 10 \\
\hline \multirow{2}{*}{ LO Nitzschia interfrigidaria } & A & $2.8(\mathrm{C} 2 \mathrm{AN}-1)$ & 2,4 \\
\hline & & $2.6-2.7(\mathrm{C} 2 \mathrm{AN}-1)$ & 10 \\
\hline \multirow{2}{*}{ FO C. vulnificus } & A & $3.1(-)$ & 2 \\
\hline & & $2.6-2.7(\mathrm{C} 2 \mathrm{AN}-1)$ & 10 \\
\hline FO N. weaveri & A & 3.1 (C2AR-2) & 8,10 \\
\hline FO $N$. interfrigidaria & $\mathrm{A}, \mathrm{B}, \mathrm{C}$ & 3.6 (C2AR-3) & 3 \\
\hline FO Nitzschia barronii & $\mathrm{A}, \mathrm{B}, \mathrm{C}$ & $4.0-4.2(\mathrm{C} 3 \mathrm{R}-1)$ & 10 \\
\hline \multirow[t]{2}{*}{ FO Rouxia heteropolara } & & $4.3(\mathrm{C} 3 \mathrm{~N}-2 / \mathrm{CN}-3)$ & 3 \\
\hline & & $3.75-3.8(\mathrm{C} 2 \mathrm{AR}-3)$ & 10 \\
\hline \multirow[t]{2}{*}{ FO Thalassiosira inura } & B & $4.45(\mathrm{C} 3 \mathrm{~N}-3)$ & 3 \\
\hline & & $4.5(\mathrm{C} 3 \mathrm{AR}-2)$ & 10 \\
\hline LO Denticulopsis hustedtii & A & $4.5(C 3 R-4)$ & 2 \\
\hline FO Thalassiosira oestrupii & C & $5.1(-)$ & 6,10 \\
\hline \multirow[t]{2}{*}{ FO Thalassiosira torokina } & $\mathrm{C}$ & 7.7 (C4R-3) & 3 \\
\hline & & $7.9(-)$ & 10 \\
\hline FO Cosmiodiscus intersectus & C & 7.9 (C4) & 3,10 \\
\hline FO Asteromphalus kennettii & B & $9.6(\mathrm{C} 5 \mathrm{~N}-1)$ & 3,10 \\
\hline LCO Denticulopsis dimorpha & $\mathrm{c}$ & $10.1-10.2(\mathrm{C} 5 \mathrm{~N}-1)$ & 10 \\
\hline FO Actinocyclus fryxellae & C & $10.5(-)$ & 10 \\
\hline \multirow[t]{2}{*}{ LO Denticulopsis praedimorpha } & & 10.5 (C5R-1) & 2 \\
\hline & & $10.1-11.0(\mathrm{C} 5 \mathrm{~N}-3)$ & 10 \\
\hline FO Nitzschia praecurta & B & $10.6(\mathrm{C} 5 \mathrm{~N}-2)$ & 3 \\
\hline \multirow[t]{2}{*}{ LO Nitzschia denticuloides } & A & 11.5 (C5R-3) & 3 \\
\hline & & 11.4 (C5R-3) & 10 \\
\hline \multirow[t]{2}{*}{ FO D. dimorpha } & C & 12.4 (C5AR-2) & 3 \\
\hline & & $11.9-12.0(\mathrm{C} 5 \mathrm{AN}-2)$ & 10 \\
\hline \multirow[t]{2}{*}{ FO $D$. praedimorpha } & $\mathrm{B}, \mathrm{C}$ & $12.6(\mathrm{C} 5 \mathrm{AN}-4)$ & 3 \\
\hline & & $12.0-12.5(\mathrm{C} 5 \mathrm{AN}-2 / \mathrm{C} 5 \mathrm{AN}-3)$ & 10 \\
\hline FO N. denticuloides & B, C & $13.5(\mathrm{C} 5 \mathrm{AN}-7)$ & 3,8 \\
\hline LO Nitzschia grossepunctata & A & $13.5(\mathrm{C} 5 \mathrm{AN}-7)$ & 3 \\
\hline
\end{tabular}

and Burckle recognized the usefulness of the last occurrence of C. vulnificus as a stratigraphic event, they chose to redefine the base of this zone at the last occurrence of Cosmiodiscus insignis. This zone correlates with the upper portion of the $T$. kolbei Zone as defined by Gersonde and Burckle (1990). This zone also correlates with the Coscinodiscus kolbei/Rhizosolenia barboi Zone of Ciesielski (1983).

\section{NSOD Zone 17 (Coscinodiscus vulnificus Partial-Range Zone)}

Top. Last occurrence of Coscinodiscus vulnificus.

Base. Last occurrence of Cosmiodiscus insignis.

Author. Ciesielski (1983).

Age. 2.22-2.49 Ma. The base of this zone correlates with Subchron C2AN-1 (McCollum, 1975; Ciesielski, 1983; Gersonde and Burckle, 1990).

Discussion. This zone correlates with the lower portion of the T. kolbei Zone as defined by Gersonde and Burckle (1990).

NSOD 16 Zone (Nitzschia interfrigidaria-Cosmiodiscus insignis Partial-Range Zone)

Top. Last occurrence of Cosmiodiscus insignis.

Base. First occurrence of Nitzschia interfrigidaria.
Author. Gersonde and Burckle (1990).

Age. 2.49-3.6 Ma. The base of this zone correlates approximately with the Gauss/Matuyama boundary (C2AR-3), whereas the top correlates with the lower portion of the Olduvai Subchronozone (C2R-1; Gersonde and Burckle, 1990; Baldauf and Barron, herein).

Discussion. This zone correlates with the interval representing the Cosmiodiscus insignis through Nitzschia praeinterfrigidaria Zones of Ciesielski (1983).

\section{NSOD Zone 15 (Nitzschia barronii Partial-Range Zone)}

Top. First occurrence of Nitzschia interfrigidaria.

Base. First occurrence of Nitzschia barronii.

Author. Gersonde and Burckle (1990).

Age. 3.6-4.2 Ma. The first occurrence of $N$. interfrigidaria is calibrated to the paleomagnetic stratigraphy (Subchron C2AR-3) at Site 745 and has an estimated age of $3.6 \mathrm{Ma}$, which is in agreement with that previously determined by Gersonde and Burckle (1990). The first occurrence of $N$. barronii is calibrated to C3AN-2 (Gersonde and Burckle, 1990; Baldauf and Barron, herein) and has an estimated age of 4.0-4.2 Ma. 
Table 2 (Continued).

\begin{tabular}{|c|c|c|c|}
\hline \multirow[b]{2}{*}{ Datum } & \multirow{2}{*}{$\begin{array}{c}\text { Reference } \\
\text { for zonal/ } \\
\text { subzonal } \\
\text { marker } \\
\text { event }^{\mathrm{a}}\end{array}$} & \multicolumn{2}{|c|}{ Age $^{\mathrm{b}}$ (calibration) } \\
\hline & & (Ma) & Reference $^{c}$ \\
\hline & & $11.9-12.0(\mathrm{C} 5 \mathrm{AN}-2)$ & 10 \\
\hline FCO D. hustedtii & & $13.7(-)$ & 9 \\
\hline FO D. hustedtii & B, C & $14.2(\mathrm{C} 5 \mathrm{AN}-8)$ & 3 \\
\hline FO N. grossepunctata & $\mathrm{B}, \mathrm{C}$ & 15.3 (C5BR-2) & 3 \\
\hline \multirow[t]{2}{*}{ LO Nitzschia maleinterpretaria } & B & $15.6(-)$ & 9 \\
\hline & & $14.6(\mathrm{C} 5 \mathrm{ADN})$ & 3 \\
\hline $\mathrm{FO} A$. ingens & $\mathrm{C}$ & $16.4(-)$ & 10 \\
\hline FO Denticulopsis maccollumii & B, C & $\begin{array}{l}<16.6(-) \\
17.2(-)\end{array}$ & $\begin{array}{l}3 \\
10\end{array}$ \\
\hline \multirow[t]{2}{*}{ FO Crucidenticulopsis nicobarica } & $\mathrm{C}$ & $17.8(\mathrm{CSDN}-1)$ & 9 \\
\hline & & $17.6-17.8(-)$ & 10 \\
\hline \multirow[t]{2}{*}{ FO $N$. maleinterpretaria } & & $18.8(-)$ & 9 \\
\hline & & $20.2(-)$ & 3 \\
\hline \multirow[t]{2}{*}{ FO Thalassiosira fraga } & C & $19.9(-)$ & 9 \\
\hline & & $20.3-20.4(\mathrm{C} 6 \mathrm{R}-1)$ & 10 \\
\hline FO Thalassiosira spumellaroides & B, C & $20.5(-)$ & 3 \\
\hline FO Thalassiosira spinosa & & $24.0-24.5(-)$ & 10 \\
\hline \multirow[t]{2}{*}{ LO Rocella gelida } & & $22.7(-)$ & 9 \\
\hline & & $21.8-22.1(\mathrm{C} 6 \mathrm{AAN}-1)$ & 10 \\
\hline \multirow[t]{3}{*}{ FO R. gelida } & $\mathrm{C}$ & $24.5(-)$ & 9 \\
\hline & & $26.0-26.2(\mathrm{C} 7 \mathrm{R}-2)$ & 8 \\
\hline & & $27.4-27.6(\mathrm{C} 8 \mathrm{~N}-2)$ & 10 \\
\hline \multirow[t]{2}{*}{ FO Rocella vigilans } & $\mathrm{C}$ & $32.0(-)$ & 7 \\
\hline & & $31.8-32.3(\mathrm{C} 11 \mathrm{~N})$ & 10 \\
\hline FO Synedra jouseana & & $32.4-32.7(\mathrm{C} 12 \mathrm{~N})$ & 10 \\
\hline LO Rhizosolenia oligocenica & & 33.3-33.4 (C12R) & 10 \\
\hline FO $R$. oligocenica & & $35.9-36.0(\mathrm{C} 13 \mathrm{R}-2)$ & 10 \\
\hline
\end{tabular}

Note: $\mathrm{LCO}=$ last common occurrence LO = last occurrence; $\mathrm{FO}=$ first occurrence.

${ }^{\mathrm{a}} \mathrm{A}=$ Ciesielski, 1983; $\mathrm{B}=$ Gersonde and Burckle, in press; $\mathrm{C}=$ this paper.

$\mathrm{b}$ Ages adjusted to Berggren et al. (1985) magnetic polarity time scale.

c $_{1}=$ Burckle et al., 1978; 2 = Ciesielski, 1983; 3 = Gersonde and Burckle, in press; $4=$ Weaver and Gombos, 1981; 5 = McCollum, 1975; 6 = Baldauf, 1985; 7 = Harwood et al., $1989 ; 8$ = Fenner, in Ciesielski, Kristoffersen, et al., 1988; $9=$ Barron, 1985; $10=$ this paper.

Discussion. Weaver and Gombos (1981) and Ciesielski (1983) defined the Nitzschia angulata Zone as the interval from the first occurrence of $N$. angulata to the first occurrence of $N$. interfrigidaria. Since then, Gersonde (in press) separated Pliocene forms referred to Nitzschia angulata (O'Meara) Hasle from N. angulata s. str. and renamed them $N$. barronii. To adhere to this taxonomic revision the base of the $N$. angulata Zone was redefined by the first occurrence of $N$. barronii, and the zone was renamed the $N$. barronii Zone by Gersonde and Burckle (1990). In this paper we do not distinguish between $N$. angulata (O'Meara) Hasle and $N$. barronii Gersonde.

\section{NSOD Zone 14 (Thalassiosira inura Partial-Range Zone)}

Top. First occurrence of Nitzschia barronii.

Base. First occurrence of Thalassiosira inura.

Author. Gersonde and Burckle (1990).

Age. 4.2-4.47 Ma. The base of this zone at Site 745 correlates with Subchron C3AR-2 (Baldauf and Barron, herein).

Discussion. This zone correlates with the Nitzschia praeinterfrigidaria and upper Denticulopsis hustedtii Zones of McCollum (1975) and to the Nitzschia reinholdii and upper D. hustedtii Zones of Ciesielski (1983).

\section{NSOD Zone 13 (Thalassiosira oestrupii Partial-Range Zone)}

Top. First occurrence of Thalassiosira inura.

Base. First occurrence of Thalassiosira oestrupii.

Author. Baldauf and Barron (herein).
Age. 4.47-5.1 Ma. The base is dated at 5.1 Ma (Baldauf and Barron, herein).

Discussion. The first occurrence of $T$. oestrupii provides a means to correlate the stratigraphy developed for the high latitudes with that for the low latitudes. Similar to its occurrence in the Kerguelen Plateau region, the first occurrence of $T$. oestrupii in the low latitudes is dated as $5.1 \mathrm{Ma}$ by Baldauf (1985). This zone correlates with the uppermost portions of the Cosmiodiscus intersectus Zone (Gersonde and Burckle, 1990) and the Denticulopsis hustedtii Zones (Ciesielski, 1983; Weaver and Gombos, 1981; McCollum, 1975).

\section{NSOD Zone 12 (Thalassiosira torokina Partial-Range Zone)}

Top. First occurrence of Thalassiosira oestrupii.

Base. First occurrence of Thalassiosira torokina.

Author. Baldauf and Barron (herein).

Age. 5.1-7.9 Ma. The first occurrence of Thalassiosira torokina lies in the reversed event below the three normal events of Chron C3 (C3N-3) in Hole 746A. Gersonde and Burckle (1990) reported a similar correlation in Leg 113 Hole 689B.

Discussion. This zone correlates with the lower portions of the Cosmiodiscus intersectus Zone of Gersonde and Burckle (1990) and the D. hustedtii Zones of Ciesielski (1983), Weaver and Gombos (1981), and McCollum (1975).

\section{NSOD Zone 11 (Actinocyclus fryxellae Interval Zone)}

Top. First occurrence of Thalassiosira torokina. Base. First occurrence of Actinocyclus fryxellae. 
Table 3. Summary of diatom biostratigraphic results from Leg 119 showing the placement of boundaries of the Neogene Southern Ocean diatom (NSOD) zones, stratigraphic events and age estimates, and stratigraphic constraints for Sites 736, 737, 744, 745, and 746.

\begin{tabular}{|c|c|c|c|c|c|c|c|}
\hline $\begin{array}{l}\text { NSOD } \\
\text { zone }\end{array}$ & Datum & $\begin{array}{l}\text { Age } \\
\text { (Ma) }\end{array}$ & $\begin{array}{c}\text { Holes } \\
736 \mathrm{~A} / 736 \mathrm{C}\end{array}$ & $\begin{array}{c}\text { Holes } \\
737 \mathrm{~A} / 737 \mathrm{~B}\end{array}$ & $\begin{array}{l}\text { Hole } \\
744 \mathrm{~A}\end{array}$ & $\begin{array}{l}\text { Hole } \\
744 \mathrm{~B}\end{array}$ & $\begin{array}{c}\text { Holes } \\
745 \mathrm{~B} / 746 \mathrm{~A}\end{array}$ \\
\hline 21 & LCO $H$. karstenii & 0.195 & $736 \mathrm{~A}-1 \mathrm{H}-5,80 / 2 \mathrm{H}-2,80$ & & & & \\
\hline 20 & LO A. ingens & 0.62 & $736 \mathrm{~A}-4 \mathrm{H}-1,80 / 4 \mathrm{H}-3,80$ & & & $1 \mathrm{H}-1,8 / 1 \mathrm{H}-\mathrm{CC}$ & $745 \mathrm{~B}-3 \mathrm{H}-\mathrm{CC} / 4 \mathrm{H}-\mathrm{CC}$ \\
\hline 20 & LO C. elliptopora & 0.65 & $736 \mathrm{~A}-8 \mathrm{H}-4,80 / 8 \mathrm{H}-\mathrm{CC}$ & & & $1 \mathrm{H}-1,8 / 1 \mathrm{H}-\mathrm{CC}$ & $745 \mathrm{~B}-5 \mathrm{H}-\mathrm{CC} / 6 \mathrm{H}-\mathrm{CC}$ \\
\hline 19 & LO R. barboi & 1.58 & $736 \mathrm{~A}-15 \mathrm{H}-5,57 / 15 \mathrm{H}-\mathrm{CC}$ & & & & $745 \mathrm{~B}-9 \mathrm{H}-\mathrm{CC} / 10 \mathrm{H}-\mathrm{CC}$ \\
\hline 18 & LO T. kolbei & 1.89 & $736 \mathrm{~A}-20 \mathrm{X}-2,57 / 20 \mathrm{X}-5,57$ & & $2 \mathrm{H}-2,150 / 2 \mathrm{H}-\mathrm{CC}$ & $1 \mathrm{H}-\mathrm{CC} / 2 \mathrm{H}-3,50$ & $745 \mathrm{~B}-10 \mathrm{H}-\mathrm{CC} / 11 \mathrm{H}-3$ \\
\hline 18 & FO C. elliptopora & 2.2 & $736 \mathrm{~A}-24 \mathrm{X}-\mathrm{CC} / 25 \mathrm{X}-\mathrm{CC}$ & & $2 \mathrm{H}-\mathrm{CC} / 3 \mathrm{H}-\mathrm{CC}$ & $2 \mathrm{H}-3,50 / 2 \mathrm{H}-\mathrm{CC}$ & $745 \mathrm{~B}-9 \mathrm{H}-\mathrm{CC} / 10 \mathrm{H}-\mathrm{CC}$ \\
\hline 17 & LO C. vulnificus & 2.22 & $736 \mathrm{~A}-24 \mathrm{X}-\mathrm{CC} / 25 \mathrm{X}-\mathrm{CC}$ & & $2 \mathrm{H}-2,150 / 2 \mathrm{H}-\mathrm{CC}$ & $1 \mathrm{H}-\mathrm{CC} / 2 \mathrm{H}-3,50$ & $745 \mathrm{~B}-11 \mathrm{H}-3 / 11 \mathrm{H}-6$ \\
\hline 16 & LO C. insignis & 2.5 & $736 \mathrm{~A}-27 \mathrm{X}-\mathrm{CC} / 29 \mathrm{X}-1,58$ & & & IH-CC $/ 2 \mathrm{H}-3,50$ & $745 \mathrm{~B}-12 \mathrm{H}-1 / 12 \mathrm{H}-2$ \\
\hline 16 & FO N. kerguelensis & 2.7 & $736 \mathrm{C}-4 \mathrm{R}-\mathrm{CC} / 5 \mathrm{R}-\mathrm{CC}$ & & $2 \mathrm{H}-\mathrm{CC} / 3 \mathrm{H}-\mathrm{CC}$ & $\mathrm{H}-\mathrm{CC} / 2 \mathrm{H}-3,50$ & $745 \mathrm{~B}-11 \mathrm{H}-3,11 \mathrm{H}-6$ \\
\hline 16 & FO C. vulnificus & 3.1 & $736 \mathrm{C}-10 \mathrm{R}-2,58 / 11 \mathrm{R}-2,58$ & & & $2 \mathrm{H}-3,50 / 2 \mathrm{H}-\mathrm{CC}$ & $745 \mathrm{~B}-13 \mathrm{H}-4 / 13 \mathrm{H}-7$ \\
\hline 16 & FO N. weaveri & 3.1 & & $737 \mathrm{~A}-3 \mathrm{H}-2,57 / 3 \mathrm{H}-5,57$ & & $3 \mathrm{H}-2,33 / 3 \mathrm{H}-\mathrm{CC}$ & $745 \mathrm{~B}-14 \mathrm{H}-4 / 14 \mathrm{H}-5$ \\
\hline 16 & FO N. interfrigidaria & 3.6 & & $737 \mathrm{~A}-6 \mathrm{H}-1,57 / 6 \mathrm{H}-5,57$ & & $3 \mathrm{H}-2,33 / 3 \mathrm{H}-\mathrm{CC}$ & $745 \mathrm{~B}-15 \mathrm{H}-5 / 15 \mathrm{H}-6$ \\
\hline 15 & FO N. barronii & 4.0 & & $737 \mathrm{~A}-7 \mathrm{H}-\mathrm{CC} / 8 \mathrm{H}-3,57$ & & $3 \mathrm{H}-\mathrm{CC} / 4 \mathrm{H}-1,70$ & $745 \mathrm{~B}-17 \mathrm{H}-4 / 17 \mathrm{H}-5$ \\
\hline 14 & FO $T$. inura & 4.47 & & $737 \mathrm{~A}-9 \mathrm{H}-\mathrm{CC} / 10 \mathrm{H}-4,57$ & & $3 \mathrm{H}-\mathrm{CC} / 4 \mathrm{H}-1,70$ & $745 \mathrm{~B}-20 \mathrm{H}-1 / 20 \mathrm{H}-4$ \\
\hline 13 & FO N. praeinterfrigidaria & 4.55 & & $737 \mathrm{~A}-9 \mathrm{H}-5 / 9 \mathrm{H}-\mathrm{CC}$ & $3 \mathrm{H}-\mathrm{CC} / 4 \mathrm{H}-1,40$ & $3 \mathrm{H}-\mathrm{CC} / 4 \mathrm{H}-1,70$ & $745 \mathrm{~B}-19 \mathrm{H}-1 / 19 \mathrm{H}-6$ \\
\hline 13 & FO T. oestrupii & 5.1 & & $737 \mathrm{~A}-11 \mathrm{H}-\mathrm{CC} / 12 \mathrm{H}-2,57$ & & & $745 \mathrm{~B}-21 \mathrm{H}-2 / 21 \mathrm{H}-5$ \\
\hline 12 & LO $T$, miocenica & 5.1 & & $737 \mathrm{~A}-13 \mathrm{H}-2,57 / 13 \mathrm{H}-\mathrm{CC}$ & & $3 \mathrm{H}-\mathrm{CC} / 4 \mathrm{H}-1,70$ & $745 \mathrm{~B}-23 \mathrm{H}-6 / 24 \mathrm{H}-2$ \\
\hline 12 & LO $T$. praeconvexa & 5.8 & & $737 \mathrm{~A}-17 \mathrm{H}-\mathrm{CC} / 18 \mathrm{H}-2,57$ & & $4 \mathrm{H}-1,70 / 4 \mathrm{H}-2,24$ & \\
\hline 12 & FO $T$, miocenica & 5.8 & & $737 \mathrm{~A}-19 \mathrm{X}-1,57 / 19 \mathrm{X}-\mathrm{CC}$ & & $4 \mathrm{H}-2,37 / 4 \mathrm{H}-2,90$ & $746 \mathrm{~A}-4 \mathrm{H}-1,60 / 4 \mathrm{H}-2,60$ \\
\hline 12 & LCO D. hustedtii & 6.3 & & $737 \mathrm{~A}-26 \mathrm{X}-1,57 / 26 \mathrm{X}-\mathrm{CC}$ & & $4 \mathrm{H}-2,90 / 4 \mathrm{H}-3,27$ & $746 \mathrm{~A}-5 \mathrm{H}-3,60 / 5 \mathrm{H}-4,60$ \\
\hline 12 & FO N. marina-cylindrica & 7.4 & & $737 \mathrm{~A}-27 \mathrm{X}-3,57 / 27 \mathrm{X}-\mathrm{CC}$ & & $4 \mathrm{H}-2,37 / 4 \mathrm{H}-2,90$ & $746 \mathrm{~A}-8 \mathrm{H}-3,60 / 8 \mathrm{H}-5,60$ \\
\hline 12 & FO T. torokina & 7.7 & & 737B-5R-2, $20 / 5 \mathrm{R}-4,20$ & $3 \mathrm{H}-\mathrm{CC} / 4 \mathrm{H}-1,40$ & $4 \mathrm{H}-2,37 / 4 \mathrm{H}-2,90$ & $746 \mathrm{~A}-9 \mathrm{H}-\mathrm{CC} / 10 \mathrm{H}-1,60$ \\
\hline 11 & FO C. intersectus & 7.9 & & 737A-27X-3, 57/27X-CC & & $4 \mathrm{H}-3,27 / 4 \mathrm{H}-3,60$ & $746 \mathrm{~A}-10 \mathrm{H}-2,60 / 10 \mathrm{H}-3,60$ \\
\hline 11 & LO D. dimorpha & 8.0 & & $737 \mathrm{~B}-5 \mathrm{R}-4,20 / 5 \mathrm{R}-\mathrm{CC}$ & & $4 \mathrm{H}-2,37 / 4 \mathrm{H}-2,90$ & $746 \mathrm{~A}-10 \mathrm{H}-\mathrm{CC} / 11 \mathrm{H}-1,60$ \\
\hline 11 & FO T. burckliana & 8.2 & & 737B-5R-CC $/ 6 \mathrm{R}-1,57$ & & & $746 \mathrm{~A}-6 \mathrm{H}-60 / 11 \mathrm{X}-\mathrm{CC}$ \\
\hline 11 & LO $D$. dimorpha var. areolata & 8.4 & & & $3 \mathrm{H}-\mathrm{CC} / 4 \mathrm{H}-1,59$ & $4 \mathrm{H}-2,37 / 4 \mathrm{H}-2,90$ & $746 \mathrm{~A}-11 \mathrm{X}-5,60 / 11 \mathrm{X}-6,60$ \\
\hline 11 & LO A. kennettii & 8.4 & & 737B-5R-2, 20/5R-4, 20 & $3 \mathrm{H}-\mathrm{CC} / 4 \mathrm{H}-1,59$ & $4 \mathrm{H}-2,90 / 4 \mathrm{H}-3,27$ & $746 \mathrm{~A}-11 \mathrm{X}-\mathrm{CC} / 13 \mathrm{X}-1,60$ \\
\hline 11 & FO D. dimorpha var. areolata & 9.5 & & & $4 \mathrm{H}-3,59 / 4 \mathrm{H}-4,60$ & $4 \mathrm{H}-3,60 / 4 \mathrm{H}-4,60$ & $746 \mathrm{~A}-11 \mathrm{X}-\mathrm{CC} / 13 \mathrm{X}-1,60$ \\
\hline 11 & FO A. kennettii & 9.6 & & 737B-5R-CC/6R-2, 57 & $4 \mathrm{H}-4,60 / 4 \mathrm{H}-\mathrm{CC}$ & $4 \mathrm{H}-6,60 / 4 \mathrm{H}-\mathrm{CC}$ & $746 \mathrm{~A}-14 \mathrm{X}-1,60 / 15 \mathrm{X}-1,56$ \\
\hline 11 & LCO D. dimorpha & 9.9 & & 737B-5R-CC/6R-1, 57 & $5 \mathrm{H}-1,60 / 5 \mathrm{H}-2,60$ & $5 \mathrm{H}-4,60 / 5 \mathrm{H}-5,60$ & \\
\hline 11 & FO A. fryxellae & 10.0 & & $737 \mathrm{~B}-6 \mathrm{R}-2,57 / 6 \mathrm{R}-4,57$ & $5 \mathrm{H}-3,60 / 5 \mathrm{H}-4,36$ & $5 \mathrm{H}-\mathrm{CC} / 6 \mathrm{H}-1,60$ & \\
\hline 10 & LO $D$. praedimorpha & 11.0 & & 737B-7R-1, 62/7R-CC & $\mathrm{SH}-\mathrm{CC} / 6 \mathrm{H}-\mathrm{CC}$ & $6 \mathrm{H}-6,60 / 6 \mathrm{H}-\mathrm{CC}$ & \\
\hline 10 & LO $N$. denticuloides & 11.4 & & 737B-7R-1, $62 / 7 \mathrm{R}-\mathrm{CC}$ & $5 \mathrm{H}-\mathrm{CC} / 6 \mathrm{H}-\mathrm{CC}$ & $6 \mathrm{H}-\mathrm{CC} / 7 \mathrm{H}-1,70$ & \\
\hline 10 & FO D. dimorpha & 11.9 & & $737 \mathrm{~B}-8 \mathrm{R}-2,62 / 8 \mathrm{R}-2,80$ & $6 \mathrm{H}-\mathrm{CC} / 7 \mathrm{H}-1,60$ & $7 \mathrm{H}-2,70 / 7 \mathrm{H}-3,70$ & \\
\hline 9 & FO D. praedimorpha & 12.6 & & $737 \mathrm{~B}-8 \mathrm{R}-2,80 / 8 \mathrm{R}-3,10$ & $6 \mathrm{H}-\mathrm{CC} / 7 \mathrm{H}-1,60$ & $7 \mathrm{H}-3,70 / 7 \mathrm{H}-4,70$ & \\
\hline 8 & LO C. nicobarica & 12.0 & & 737B- $8 \mathrm{R}-2,80 / 8 \mathrm{R}-3,10$ & $6 \mathrm{H}-\mathrm{CC} / 7 \mathrm{H}-1,60$ & $7 \mathrm{H}-2,70 / 7 \mathrm{H}-3,70$ & \\
\hline 8 & LO N. grossepunctata & 12.0 & & $737 \mathrm{~B}-9 \mathrm{R}-2,56 / 9 \mathrm{R}-\mathrm{CC}$ & $6 \mathrm{H}-\mathrm{CC} / 7 \mathrm{H}-1,60$ & $7 \mathrm{H}-2,70 / 7 \mathrm{H}-3,70$ & \\
\hline 8 & LO $A$. ingens var. nodus & 12.3 & & $737 \mathrm{~B}-10 \mathrm{R}-1,57 / 10 \mathrm{R}-3,57$ & $6 \mathrm{H}-\mathrm{CC} / 7 \mathrm{H}-1,60$ & $7 \mathrm{H}-3,70 / 7 \mathrm{H}-4,70$ & \\
\hline 8 & FO N. denticuloides & 13.5 & & 737B-10R-3, 57/10R-CC & $7 \mathrm{H}-1,60 / 7 \mathrm{H}-2,60$ & $7 \mathrm{H}-3,70 / 7 \mathrm{H}-4,70$ & \\
\hline 7 & FCO D. hustedtii & 14.0 & & 737B-10R-3, 57/10R-CC & $7 \mathrm{H}-1,60 / 7 \mathrm{H}-2,60$ & $7 \mathrm{H}-5,70 / 7 \mathrm{H}-6,70$ & \\
\hline 7 & FO D. hustedtii & 14.2 & & 737B-10R-3, 57/10R-CC & $7 \mathrm{H}-3,60 / 7 \mathrm{H}-4,60$ & $7 \mathrm{H}-5,70 / 7 \mathrm{H}-6,70$ & \\
\hline 6 & LO $D$, maccollumii & 14.4 & & & $7 \mathrm{H}-3,60 / 7 \mathrm{H}-4,60$ & $7 \mathrm{H}-2,70 / 7 \mathrm{H}-3,70$ & \\
\hline 6 & FO D. hyalina & 15.0 & & & $7 \mathrm{H}-\mathrm{CC} / 8 \mathrm{H}-\mathrm{CC}$ & $8 \mathrm{H}-3,60 / 8 \mathrm{H}-4,60$ & \\
\hline 6 & FO $A$. ingens var. nodus & 15.0 & & & $7 \mathrm{H}-\mathrm{CC} / 8 \mathrm{H}-\mathrm{CC}$ & $8 \mathrm{H}-3,60 / 8 \mathrm{H}-4,60$ & \\
\hline 6 & FO N. grossepunctata & 15.3 & & & $7 \mathrm{H}-5,60 / 7 \mathrm{H}-\mathrm{CC}$ & $8 \mathrm{H}-5,60 / 8 \mathrm{H}-6,60$ & \\
\hline 6 & LO N. maleinterpretaria & 15.6 & & & $7 \mathrm{H}-\mathrm{CC} / 8 \mathrm{H}-\mathrm{CC}$ & $8 \mathrm{H}-5,60 / 8 \mathrm{H}-6,60$ & \\
\hline 6 & FO D. lauta & 16.0 & & & $7 \mathrm{H}-\mathrm{CC} / 8 \mathrm{H}-\mathrm{CC}$ & $8 \mathrm{H}-4,60 / 8 \mathrm{H}-5,60$ & \\
\hline 6 & FO $A$. ingens & 16.4 & & & $7 \mathrm{H}-\mathrm{CC} / 8 \mathrm{H}-\mathrm{CC}$ & $8 \mathrm{H}-4,60 / 8 \mathrm{H}-5,60$ & \\
\hline 5 & FO D. maccollumii & 17.2 & & & $9 \mathrm{H}-\mathrm{CC} / 10 \mathrm{H}-1,60$ & $8 \mathrm{H}-\mathrm{CC} / 9 \mathrm{H}-1,58$ & \\
\hline 4 & FO C. nicobarica & 17.8 & & & $9 \mathrm{H}-\mathrm{CC} / 10 \mathrm{H}-1,60$ & $9 \mathrm{H}-4,58 / 9 \mathrm{H}-5,58$ & \\
\hline 3 & FO T. fraga & $20.3-20.4$ & & & $11 \mathrm{H}-1,63 / 11 \mathrm{H}-2,63$ & & \\
\hline 2 & FO $T$. spumellaroides & $21.0-21.2$ & & & $11 \mathrm{H}-4,63 / 11 \mathrm{H}-5,63$ & & \\
\hline \multirow[t]{3}{*}{1} & FO T. spinosa & $24.0-24.5$ & & & $11 \mathrm{H}-\mathrm{CC} / 12 \mathrm{H}-1,60$ & & \\
\hline & FO $R$. gelida & $27.4-27.6$ & & & $13 \mathrm{H}-1,60 / 13 \mathrm{H}-2,60$ & & \\
\hline & FO $R$. vigilans & $31.8-32.3$ & & & $14 \mathrm{H}-2,60 / 14 \mathrm{H}-4,60$ & & \\
\hline
\end{tabular}

Note: $\mathrm{LO}=$ last occurrence, $\mathrm{LCO}=$ last common occurrence, $\mathrm{FO}=$ first occurrence, $\mathrm{FCO}=$ first common occurrence; core interval in centimeters. 


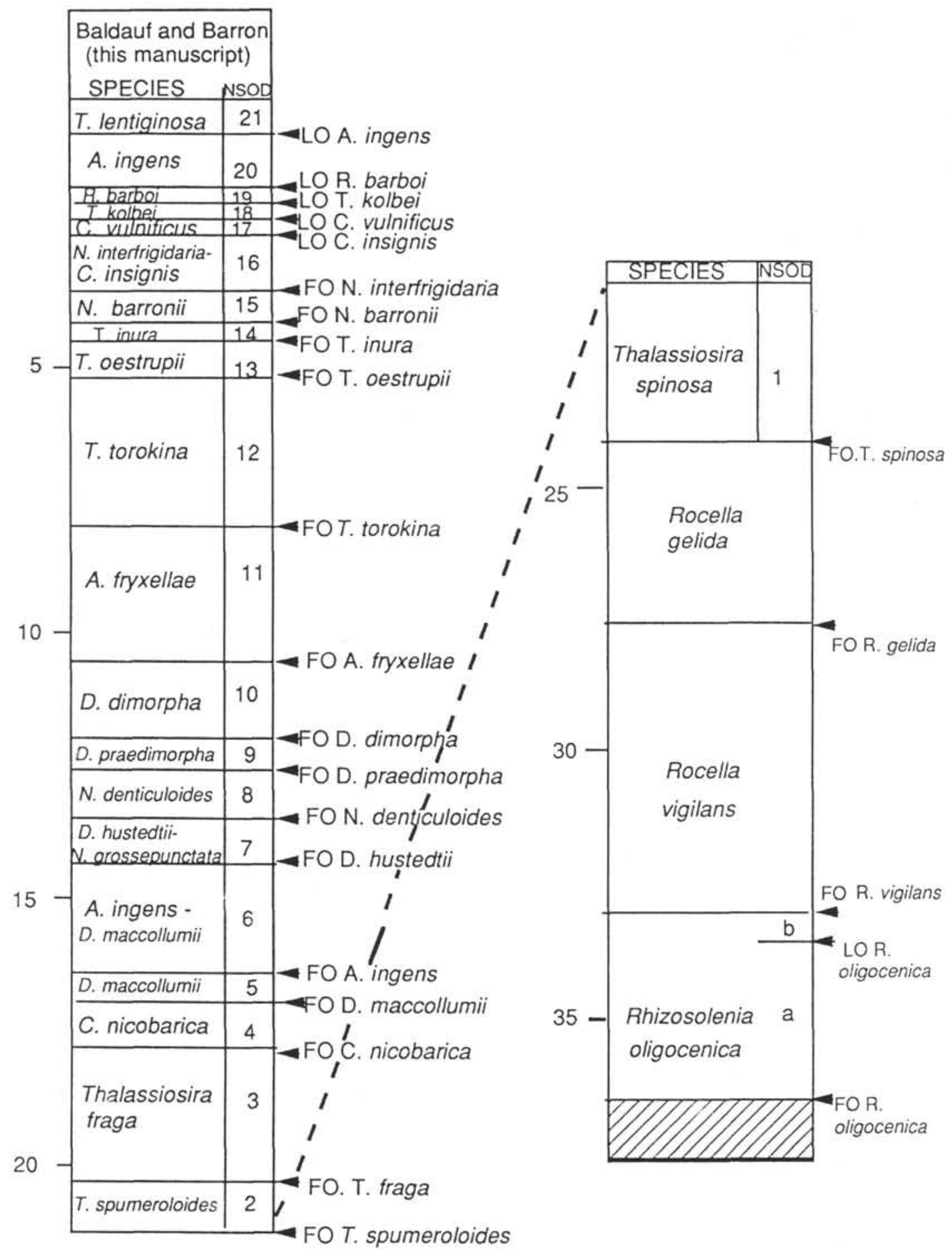

Figure 3. The Quaternary through Oligocene diatom zonation proposed in this manuscript and the marker species used to delineate the individual zonal boundaries. NSOD $=$ Neogene Southern Ocean diatom zones; FO = first occurrence; $\mathrm{LO}=$ last occurrence; $\mathrm{LCO}=$ last common occurrence.

Author. Baldauf and Barron (herein).

Age. 7.9-10.5 Ma.

Discussion. Gersonde and Burckle (1990) defined an Asteromphalus kennettii Zone as the interval between the first $A$. kennettii and the first Cosmiodiscus insignis. As subsequently discussed, A. kennettii is easily removed from assemblages by dissolution and should not be used as a biostratigraphic marker. Similarly, Cosmiodiscus intersectus can be easily confused with
Thalassiosira oliverana var. of Harwood et al. (in press), whereas as distinctive Thalassiosira torokina is a consistent member of uppermost Miocene Antarctic diatom assemblages (Harwood, 1986a; Harwood et al., in press). The first occurrence of Actinocyclus fryxellae lies immediately below the last common occurrence of $D$. dimorpha at Leg 119 Sites 737 and 744, Leg 28 Site 266 , and Leg 29 Site 278 (see "Discussion"), suggesting that the latter datum level is isochronous. This zone correlates with the 
Asteromphalus kennettii and Nitzschia praecurta Zones of Gersonde and Burckle (1990) and the Denticulopsis hustedtii-D. lauta Zones of Ciesielski (1983), Weaver and Gombos (1981), and McCollum (1975).

\section{NSOD Zone 10 (Denticulopsis dimorpha Acme Zone)}

Top. Last common occurrence of Denticulopsis dimorpha.

Base. First occurrence of Denticulopsis dimorpha.

Author. Baldauf and Barron (herein).

Age. 10.5-11.9 Ma (Gersonde and Burckle, 1990).

Discussion. In Holes 737B and 744B this zone is distinguished by common to abundant occurrences of $D$. dimorpha. Gersonde and Burckle (1990) recorded similar abundance intervals in Holes 689B and 690B. Gersonde and Burckle favored using the first occurrence of Asteromphalus kennettii ( $9.6 \mathrm{Ma}$ ) as a zonal marker, but this taxon is susceptible to dissolution and unreliable. At Sites 689,737 , and 744 , the first occurrence of A. kennettii definitely lies above the last common occurrence of $D$. dimorpha, whereas at Sites 278 and 690 these datums are coincident (see "Biostratigraphic Results" and Gersonde and Burckle, 1990). This zone correlates with the upper portion of the Denticulopsis praedimorpha Zone of Gersonde and Burckle (1990), the upper portion of the Nitzschia denticuloides Zone of Ciesielski (1983), and the middle portion of the $D$. hustedtii-D. lauta Zone of McCollum (1975). This zone differs from the $D$. dimorpha Zone of Schrader (1976), which is defined as the interval from the last Denticula nicobarica (now referred to as Crucidenticula nicobarica) to the first occurrences of Coscinodiscus yabei (now referred to as Thalassiosira yabei) and Nitzschia donahuensis.

\section{NSOD Zone 9 (Denticulopsis praedimorpha Partial-Range Zone)}

Top. First occurrence of Denticulopsis dimorpha.

Base. First occurrence of Denticulopsis praedimorpha.

Author. Baldauf and Barron (herein).

Age. 11.9-12.6 Ma. The first occurrence of D. dimorpha correlates with the lower normal event of Chron C5AN-2 at Site 744. The first occurrence of $D$. praedimorpha correlates with the fourth normal event of Chron C5AN-2/C5AN-3.

Discussion. This zone correlates with the lowermost portion of the Denticulopsis praedimorpha Zone of Gersonde and Burckle (1990), the Nitzschia denticuloides Zone of Ciesielski (1983), and the lower portion of the Denticulopsis hustedtii-Denticulopsis lauta Zone of McCollum (1975).

\section{NSOD Zone 8 (Nitzschia denticuloides Partial-Range Zone)}

Top. First occurrence of Denticulopsis praedimorpha.

Base. First occurrence of Nitzschia denticuloides.

Author. Gersonde and Burckle (1990).

Age. 12.6-13.5 Ma (Gersondè and Burckle, 1990).

Discussion. NSOD Zone 8 correlates with the lower portion of the Nitzschia denticuloides Zone of Ciesielski (1983).

\section{NSOD Zone 7 (Denticulopsis hustedtii-Nitzschia grossepunctata Partial-Range Zone)}

Top. First occurrence of Nitzschia denticuloides.

Base. First occurrence of Denticulopsis hustedtii.

Author. Gersonde and Burckle (1990).

Age. 13.5-14.2 Ma (Gersonde and Burckle, 1990).

Discussion. This zone was named the $D$. hustedtii/Nitzschia grossepunctata Zone by Gersonde and Burckle (1990) in order that it would not be confused with the Denticulopsis hustedtii Zone previously discussed in literature pertaining to the Southern Ocean. This zone occurs in the late Miocene and coincides with the range of $D$. hustedtii above the last occurrence of Denticulopsis lauta. The last occurrence of Nitzschia grossepunctata approximates the top of the $D$. hustedtii/N. grossepunctata Zone as used here. Available biostratigraphic data (Gersonde and Burckle, 1990; Barron, in press) suggest that the first occurrence of $D$. hustedtii is isochronous between the North Pacific and Southern Ocean.

\section{NSOD Zone 6 (Actinocyclus ingens-Denticulopsis maccollumii Partial-Range Zone)}

Top. First occurrence of Denticulopsis hustedtii.

Base. First occurrence of Actinocyclus ingens.

Author. Baldauf and Barron (herein).

Age. 14.2-16.4 Ma (Baldauf and Barron, herein).

Discussion. Gersonde and Burckle (1990) defined the base of the Nitzschia grossepunctata and Denticulopsis maccollumii Zones at the first Nitzschia grossepunctata and the first D. maccollumii, respectively. This first species has an inconsistent first occurrence requiring that the Nitzschia grossepunctata Zone of Gersonde and Burckle (1990) be modified for use in the Leg 119 region. Therefore, we replace the $N$. grossepunctata Zone with the Actinocyclus ingens-Denticulopsis maccollumii Zone and define this latter zone as the interval from the first occurrence of $A$. ingens to the first occurrence of $D$. hustedtii. Thus this zone correlates with the $N$. grossepunctata and upper portion of the $D$. maccollumii Zones of Gersonde and Burckle (1990) and with the $N$. grossepunctata through upper portion of the Nitzschia maleinterpretaria Zones of Weaver and Gombos (1981).

\section{NSOD Zone 5 (Denticulopsis maccollumii Partial-Range Zone)}

Top. First occurrence of Actinocyclus ingens.

Base. First occurrence of Denticulopsis maccollumii.

Author. Gersonde and Burckle (1990); modified by Baldauf and Barron (herein).

Age. 16.4-17.2 Ma (Gersonde and Burckle, 1990; Baldauf and Barron, herein). The first occurrence of Denticulopsis maccollumii correlates with paleomagnetic event C5CR-3 at Site 744 and has an estimated age of $17.2 \mathrm{Ma}$. The first occurrence of $D$. maccollumii coincides with an unconformity at Sites 278 and 689. Interpolation of diatom datums at Site 266 suggests an age of approximately $17.2 \mathrm{Ma}$ for the first occurrence of $D$. maccollumii. This age is slightly older than that derived by Gersonde and Burckle (1990) at Site 690, where this datum coincides with one of the three normal events of Chron C5 (16.2-16.98 Ma).

Discussion. The D. maccollumii Zone as defined in this paper correlates with the lowermost portion of the D. maccollumii Zone of Gersonde and Burckle (1990) and with the lower portion of the N. maleinterpretaria Zone of Weaver and Gombos (1981).

\section{NSOD Zone 4 (Crucidenticula nicobarica Partial-Range Zone)}

Top. First occurrence of Denticulopsis maccollumii.

Base. First occurrence of Crucidenticula nicobarica s. ampl.

Author. Barron (1983); modified by Baldauf and Barron (herein).

Age. 17.2-17.8 Ma.

Discussion. Species tabulated as $C$. nicobarica s. ampl. in this report include $C$. paranicobarica Akiba and Yanagisawa and $C$. sawamurae Yanagisawa and Akiba (proposed). If the proposed species Crucidenticula sawamurae Yanagisawa and Akiba (in press) is accepted, this zone will be renamed the $C$. sawamurae Zone and the first occurrence of $C$. sawamurae will mark its base. This zone is comparable to an equatorial Pacific zone proposed by Barron (1983). However, the definition of the top of the zone is modified here. This zone correlates with the upper portion of the Nitzschia maleinterpretaria Zone of Gersonde and Burckle (1990). The first occurrence of $C$. nicobarica s. ampl. occurs in paleomagnetic event C5DN-1 in Hole 744A. This biostratigraphic event is isochronous with its occurrence in the equatorial Pacific (Barron, 1985a). 


\section{Zone NSOD 3 (Thalassiosira fraga Partial-Range Zone)}

Top. First occurrence of Crucidenticula nicobarica s. ampl.

Base. First occurrence of Thalassiosira fraga.

Author. Barron (1985b); modified by Baldauf and Barron (herein).

Age. $17.8-20.3 \mathrm{Ma}$.

Discussion. The $T$. fraga Zone of Barron (1985b) was defined for use in the North Pacific. It is modified here for use in the Southern Ocean. The first occurrence of $T$. fraga is a biostratigraphically useful datum level in the Southern Ocean (Gersonde and Burckle, 1990) as well as in the equatorial and North Pacific (Barron, 1983, 1985b) and is proposed here to mark the top of this zone.

\section{NSOD Zone 2 (Thalassiosira spumellaroides Partial-Range Zone)}

Top. First occurrence of Thalassiosira fraga.

Base. First occurrence of Thalassiosira spumellaroides.

Author. Schrader (1976); Gersonde and Burckle (1990); modified by Baldauf and Barron (herein).

Age. 20.3-21.0/21.2 Ma. In Hole 744A, the first occurrence of $T$. fraga is placed immediately below paleomagnetic event $\mathrm{C} 6 \mathrm{~N}$ (Baldauf and Barron, herein).

Discussion. The first occurrence of $T$. spumellaroides is a useful biostratigraphic datum in the Southern Ocean (Gersonde and Burckle, 1990) and in the equatorial Pacific (Barron, 1983). This datum level occurs in paleomagnetic event $\mathrm{C} 6 \mathrm{~N}-1$ in Hole $744 \mathrm{~A}$. Use of the first occurrence of Nitzschia maleinterpretaria to define the top of the zone as employed by Gersonde and Burckle (1990) is discouraged by the sporadic occurrence of that species in Hole $744 \mathrm{~A}$ and by its susceptibility to dissolution. The $T$. spumellaroides Zone as used here differs from the $T$. spumellaroides Zone of Schrader (1976), which represents the interval from the last occurrence of Thalassiosira spinosa and the first occurrence of Azpeitia endoi to the last occurrence of $T$. spumellaroides. NSOD Zone 2 correlates with the lower portion of the $N$. maleinterpretaria and the T. spumellaroides Zones of Gersonde and Burckle (1990).

\section{NSOD Zone 1 (Thalassiosira spinosa Partial-Range Zone)}

Top. First occurrence of Thalassiosira spumellaroides.

Base. First occurrence of Thalassiosira spinosa.

Author. Barron (1985b); modified Baldauf and Barron (herein).

Age. 21.0/21.2-24.0/24.5 Ma.

Discussion. The first occurrence of $T$. spinosa has an estimated age between 24.0 and $24.5 \mathrm{Ma}$ based on correlation of this event to the magnetic stratigraphy at Site 744 . This estimated age approximates that assigned to this event in the equatorial Pacific. This zone differs from the T. spinosa Zone of Schrader (1976), which represents the interval from the first occurrence of Navicula sp. 1 of Schrader (1976) to the last occurrence of $T$. spinosa and the first occurrence of Coscinodiscus endoi.

\section{Rocella gelida Partial-Range Zone}

Top. First occurrence of Thalassiosira spinosa.

Base. First occurrence of Rocella gelida.

Author. Bukry and Foster (1974), modified by Baldauf and Barron (herein).

Age. $24.0 / 24.5-27.6 \mathrm{Ma}$.

Discussion. The first occurrence of $R$. gelida correlates with C8N-2 in Hole 744A and has an age between 27.4 and 27.6 Ma.

\section{Rocella vigilans Partial-Range Zone}

Top. First occurrence of Rocella gelida.

Base. First occurrence of Rocella vigilans.
Author. Jousé (1974), modified by Baldauf and Barron (herein).

Age. 27.4-32.7/32.9 Ma. Interval of paleomagnetic Chron C11-C12 (basal age estimated at $32 \mathrm{Ma}$ by Harwood et al., in press) to the reversed event above anomaly $7 \mathrm{~A}$ (J. Fenner in Ciesielski, Kristoffersen, et al., 1988).

Discussion. This zone is equivalent to the Rocella vigilans and Bogorovia veniamini Zones of Fenner (1985), to the $R$. vigilans and Triceratium groningensis Zones of Gombos and Ciesielski (1983), and to the Lisitzinia ornata Zone and Subzone b of the Synedra jouseana Zone of Harwood (1986b).

\section{Rhizosolenia oligocenica Partial-Range Zone}

Top. First occurrence of Rocella vigilans.

Base. First occurrence of Rhizosolenia oligocenica.

Subzone. The last occurrence of Rhizosolenia oligocenica marks the Subzone a/b boundary.

Author. Gombos and Ciesielski (1983); modified by Baldauf and Barron (herein).

Age. $32.7 / 32.9-36.0 \mathrm{Ma}$. Subzone a/b boundary at $33.3 \mathrm{Ma}$. The first occurrence of $R$. oligocenica at Site 744 approximates the interval representing the lower portion of magnetic Subchron C13C.

Discussion. Rhizosolenia oligocenica of Schrader (1976) is equivalent to Rhizosolenia gravida and has taxonomic priority over this latter species. Gombos and Ciesielski (1983) previously defined the Rhizosolenia gravida Zone as the interval from the last occurrence of $R$. gravida ( $=R$. oligocenica) stratigraphically down to the first occurrence of Asteromphalus oligocenicus. Fenner $(1984,1985)$ redefined this zone as the interval from the first occurrence of Rhizosolenia antarctica to the first occurrence of $R$. gravida $(=R$. oligocenica $)$. Unfortunately, $R$. antarctica has a sporadic occurrence at Site 744 , which prohibits recognition of the top of this zone as defined by Fenner $(1984,1985)$. Instead, the $R$. gravida and $R$. antarctica Zones of Fenner are combined to define the $R$. oligocenica Zone used in this paper. Although the last occurrence of $R$. oligocenica is abrupt and apparently stratigraphically equivalent in Hole 744A (see the following) and Leg 71 Hole 513A (Gombos and Ciesielski, 1983), Fenner (1985) recorded the last occurrence of $R$. gravida $(=R$. oligocenica) stratigraphically higher at DSDP Leg 38 Site 350 (into the $R$. gelida Zone), questioning the reliability of this event as a regionally useful event. However, based on its abrupt occurrence at Site 744 this event is locally useful and is used to delineate the Subzone a/Subzone b boundary. Synedra jouseana has its first occurrence in the upper portion of Subzone b.

\section{BIOSTRATIGRAPHIC RESULTS}

The biostratigraphic discussion in this paper focuses on the post-cruise analysis completed for Sites $736,737,739$, and 744 through 746 (see stratigraphic summary, Table 3) and incorporates shipboard results only where necessary. The paucity of diatoms from Sites 740 through 743 and the limited stratigraphic occurrence of diatoms from Site 738 precluded incorporating these sites in the subsequent post-cruise studies. The reader is referred to Barron, Larsen, et al. (1989) for additional discussion pertaining to shipboard results from these sites and to the Appendix for discussion of the taxonomic concepts used herein.

\section{Kerguelen Plateau}

\section{Site 736}

Site 736 is on the northern Kerguelen Plateau in a water depth of $629 \mathrm{~m}$ (Table 1 and Fig. 1). Holes 736A through 736C were cored at this site, with a total of 51 cores recovered. The combined stratigraphic sequence recovered consists of $371 \mathrm{~m}$ of predomi- 
nately upper lower Pliocene to Holocene diatom ooze. Diatoms are present in all samples examined from the three holes cored at this site. Abundance and preservation vary from sample to sample, but the diatoms are generally abundant and well preserved. The diatom assemblage present is typical for the Pliocene through Holocene Southern Ocean and is dominated by several species, including Actinocyclus ingens, Nitzschia kerguelensis, Nitzschia ritscheri, and Thalassiothrix longissima. The occurrence of selected species, including the stratigraphically important taxa, in Holes $736 \mathrm{~A}$ and $736 \mathrm{C}$ is given in Table 4 . The absence of a reliable magnetic signature precludes calibrating any stratigraphic events at this site to magnetostratigraphy.

The last occurrence of Actinocyclus ingens in Sample 119$736 \mathrm{~A}-4 \mathrm{H}-3,80-82 \mathrm{~cm}$, allows Cores $119-736 \mathrm{~A}-1 \mathrm{H}$ through $119-$ $736 \mathrm{~A}-3 \mathrm{H}$ and the upper portion of Core $119-736 \mathrm{~A}-4 \mathrm{H}$ to be assigned to NSOD Zone 21 . One specimen of A, ingens occurs in Sample $119-736 \mathrm{~A}-4 \mathrm{H}-1,80-82 \mathrm{~cm}$, but this single specimen is assumed to be a result of reworking. The diatom flora observed in this interval is characterized by Eucampia antarctica, N. kerguelensis, $N$. ritscheri, Thalassiosira kolbei, Thalassosira oestrupii, and Thalassiothrix longissima. Hemidiscus karstenii occurs somewhat consistently in the interval from Samples 119. $736 \mathrm{~A}-2 \mathrm{H}-2,80-82 \mathrm{~cm}$, down to $119-736 \mathrm{~A}-4 \mathrm{H}-3,80-82 \mathrm{~cm}$. The acme of this species was used previously to stratigraphically subdivide the Quaternary (Ciesielski, 1983).

NSOD Zone 20 can be recognized in the interval from Samples $119-736 \mathrm{~A}-4 \mathrm{H}-3,80-82 \mathrm{~cm}$, to $119-736 \mathrm{~A}-15 \mathrm{H}-5,57-59 \mathrm{~cm}$. However, the sporadic occurrence of Rhizosolenia barboi at Site 736 limits its usefulness. The last occurrence of this species, and therefore the base of NSOD Zone 20, is tentatively placed in Sample 119-736A-15H-CC. The last occurrence of Coscinodiscus elliptopora is placed in the uppermost portion of this zone, in Sample 119-736A-8H-CC.

The interval from Samples 119-736A-15H-CC, to 119-736A$20 \mathrm{H}-2,57-59 \mathrm{~cm}$, is assigned to NSOD Zone 19 based on the last occurrences of $R$. barboi in the former sample and of $T$. kolbe $i$ in Sample 119-736A-20H-5, $57 \mathrm{~cm}$. NSOD Zone 18 extends from Samples $119-736 \mathrm{~A}-20 \mathrm{H}-5,57 \mathrm{~cm}$, to $119-736 \mathrm{~A}-24-\mathrm{CC}$. The last occurrence of Coscinodiscus vulnificus is in Sample 119-736A$25 \mathrm{H}-\mathrm{CC}$. The first occurrence of Coscinodiscus elliptopora is placed between core-catcher samples of 119-736A-24H and 119$736 \mathrm{~A}-25 \mathrm{H}$ and may be a useful secondary marker to delineate the base of this zone. Cores $119-736 \mathrm{~A}-26 \mathrm{H}$ and $119-736 \mathrm{~A}-27 \mathrm{H}$ are placed in NSOD Zone 17 based on the last occurrence of Cosmiodiscus insignis in Sample 119-736A-29H-1, 58-60 cm (no sample was available from Core 119-736A-28H).

The remaining interval from Hole 736A (Cores 119-736A-29H and $119-736 \mathrm{~A}-30 \mathrm{H}$ ) and Hole $736 \mathrm{C}$ (Cores $119-736 \mathrm{C}-2 \mathrm{H}$ through $119-736 \mathrm{C}-18 \mathrm{H}$ ) is placed in NSOD Zone 16 based on the continuous occurrence of Nitzschia interfrigidaria and Nitzschia weaveri throughout these intervals. This suggests that the interval cored is stratigraphically above the first occurrence of both of these species, indicating that the base of the sequence is younger than $3.6 \mathrm{Ma}$.

\section{Site 737}

Site 737 is positioned $100 \mathrm{~km}$ southeast of Site 736 , in a water depth of $564 \mathrm{~m}$ (Table 1 and Fig. 1). A 715.5-m-thick middle Eocene to lower Pliocene sequence was recovered from the two holes cored at Site 737 . With the exception of a thin $(<1 \mathrm{~m})$ Quaternary veneer, Site 737 begins in the middle part of the Pliocene, near the stratigraphic level where coring was terminated at Site 736.

Diatoms are consistently present in the middle Miocene through lower Pliocene and Quaternary sediments recovered from this site. With the exception of the uppermost samples, diatoms were not observed in the middle Eocene to lower Oligocene sequence. The flora observed in the younger stratigraphic interval is diverse and generally well preserved. Although diatoms are present in the Oligocene interval, their occurrence is sporadic, and the observed specimens are poorly preserved. The occurrence of selected species, including stratigraphically important diatom taxa, in Holes 737 A and 737B is given in Tables 5 and 6, respectively.

The uppermost $50 \mathrm{~cm}$ of Hole 737A is placed in NSOD Zone 21 based on the occurrence of typical Quaternary species such as Thalassiosira lentiginosa, Nitzschia kerguelensis, and Thalassiosira inura without late Pliocene-Pleistocene species such as Actinocyclus ingens, Thalassiosira kolbei, Rhizosolenia barboi, or Coscinodiscus elliptopora. The occurrence of abundant and well-preserved specimens in this interval and their presence at nearby Site 736 suggest that these Pliocene-Pleistocene forms are not preservationally or ecologically excluded from this interval.

The uppermost $50 \mathrm{~cm}$ of Hole $737 \mathrm{~A}$ is interpreted as a surficial layer resting unconformably on a sequence dated as Pliocene on the basis of diatom biostratigraphy. The hiatus spanning most of the late Pliocene and the Quaternary (NSOD Zones 20 through 17) approximates the level of Sample $119-737 \mathrm{~A}-1 \mathrm{H}-1,50 \mathrm{~cm}$. It should be noted that stratigraphic placement of this hiatus is tentative as a result of drilling disturbance and bioturbation present throughout Section 119-737A-1H-1. Examination of selected burrows indicates the occurrence of either a mixed upper Pliocene and Quaternary assemblage or a specific Quaternary assemblage.

NSOD Zone 16 can be recognized from Samples 119-737A$1 \mathrm{H}-\mathrm{CC}$ to $119-737 \mathrm{~A}-6 \mathrm{H}-1,57-59 \mathrm{~cm}$, where the first occurrence of Nitzschia interfrigidaria is recorded (Table 5). Sample 119$737 \mathrm{~A}-1 \mathrm{H}-\mathrm{CC}$, the youngest sample examined below the thin Quaternary glauconitic sand at the top of the hole, contains Nitzschia interfrigidaria, Nitzschia praeinterfrigidaria, Nitzschia weaveri, Thalassiosira kolbei, and Thalassiosira lentiginosa and lacks Cosmiodiscus insignis and Coscinodiscus vulnificus (Table 5). This suggests a correlation in which the middle portion of NSOD Zone 16 is equivalent to about $2.8 \mathrm{Ma}$ based on the ranges of these species at Site 745 (see the following). The first occurrence of Nitzschia weaveri (3.1 Ma) in Sample 119-737A-3H-2, 57-59 cm, appears to be a useful datum in Hole 737A, although Gersonde and Burckle (1990) challenged the isochroneity of this datum in the Southern Ocean.

NSOD Zone 15, defined as the interval from the first occurrence of Nitzschia barronii to the first $N$. interfrigidaria, occurs from Samples 119-737A-6H-5, $57 \mathrm{~cm}$, to 119-737A-7H-CC. Nitzschia barronii as used here (incorporating both Nitzschia angulata and $N$. barronii) is viewed as a primary stratigraphic marker that has an abrupt stratigraphic appearance at $4.2 \mathrm{Ma}$ based on its range at the other Leg 119 sites.

Samples 119-737A-8H-3, 57-59 cm, and 119-737A-9H-CC are assigned to NSOD Zone 14, based on the range of $T$. inura s. ampl. below the first occurrence of $N$. barronii (Table 5). Important datums occurring within NSOD Zone 14 in Hole 737A include the last occurrence of Rouxia heteropolara in Sample 119-737A-8H-6, 57-59 cm, and the first occurrence of Nitzschia praeinterfrigidaria in Sample 119-737A-9H-5, 57-59 cm (Table 5). These two datums also occur in the same sequence within NSOD Zone 14 at Site 745 (see the following).

The interval from Samples 119-737A-10H-4, $57 \mathrm{~cm}$, to 119 $737 \mathrm{~A}-11 \mathrm{H}-\mathrm{CC}$ is assigned to NSOD Zone 13 based on the occurrence of Thalassiosira oestrupii stratigraphically below the occurrence of $T$. inura. Thalassiosira oestrupii first occurs in Sample 119-737A-11H-CC. Although this zone represents a short interval (duration of $\sim 0.6$ m.y.) of geologic time, it is a critical interval for correlating sequences in the Southern Ocean with those in lower latitudes. 
NSOD Zone 12 is recognized from Samples 119-737A-12H-2, $57 \mathrm{~cm}$, (immediately below the first occurrence of $T$. oestrupii; Tables 5 and 6) through 119-737 A-28H-CC (the base of Hole $737 \mathrm{~A}$ ) to $119-737 \mathrm{~B}-5 \mathrm{H}-2,20-22 \mathrm{~cm}$, where Thalassiosira torokina first occurs. The occurrences of $T$. torokina and Cosmiodiscus intersectus in Sample 119-737B-6H-2, 57-59 cm, are regarded as downhole contamination.

An interval from Cores 119-737A-18H through 119-737B-5H, from 157.0 to $263.2 \mathrm{~m}$ below seafloor (mbsf), in lower NSOD Zone 11 and uppermost NSOD Zone 10 contains a sequence of biostratigraphic events of warm to warm-temperate diatoms typical of the low to low-middle latitudes. The sequence of these events occurs in the same sequence as one would expect in low-latitude oceans (ages for equatorial Pacific after Barron, in press):

last occurrence of Thalassiosira praeconvexa $(5.8 \mathrm{Ma})$ in Sample 119-737A-18H-2, 57-59 cm;

first occurrence of Thalassiosira miocenica (6.1 Ma) in Sample 119-737A-19H-1, 57-59 cm;

first occurrence of Nitzschia miocenica (6.8 Ma) in Sample 119-737A-21H-CC;

last occurrence of Thalassiosira burckliana (7.0 Ma) in Sample $119-737 \mathrm{~A}-23 \mathrm{H}-2,57-59 \mathrm{~cm}$;

first occurrences of Nitzschia marina and Nitzschia cylindrica (7.4 and 7.55 Ma, respectively) in Sample 119-737A-27H-3, $57-59 \mathrm{~cm}$; and

first occurrence of Thalassiosira burckliana (8.2 Ma) in Sample 119-737B-5H-CC.

The occurrences of warm to warm-temperate taxa are not totally consistent throughout this interval, especially in Cores $119-737 \mathrm{~A}-23 \mathrm{H}$ through $119-737 \mathrm{~A}-26 \mathrm{H}$, but neither are the occurrences of cooler water taxa such as Cosmiodiscus intersectus, Eucampia antarctica, or Thalassiosira torokina (Table 5). Within this possibly warm interval, the last common occurrence of Denticulopsis hustedtii occurs in Sample 119-737A-27H-1, 57-59 $\mathrm{cm}$, (about 7.3 Ma) coincident with the top of lithologic Unit II, a diatom-nannofossil ooze (Barron, Larsen, et al., 1989).

Within NSOD Zone 12 the Miocene/Pliocene boundary may lie within the lower part of Core 119-737A-13H based on the first occurrence of Thalassiosira praeoestrupii (5.4 Ma) in Sample 119-737A-13H-CC (a marker for the Miocene/Pliocene in California; Dumont et al., 1986) and the last occurrence of Thalassiosira miocenica in the same sample. T. miocenica is a warm to warm-temperate species that disappears at $5.35 \mathrm{Ma}$ in the middlelatitude northwest Pacific (Koizumi and Tanimura, 1985), and such an age is suggested in Hole 737A for this event based on its near coincidence with the first occurrence of $T$. praeoestrupii.

No stratigraphically useful datums are apparent in NSOD Zone 13, although the last occurrence of Thalassiosira praeoestrupii in Sample 119-737A-11H-2,57-59 cm, may be a correlatable event in temperate regions of the Southern Ocean. NSOD Zone 11 can be recognized in Samples 119-737B-5H-4, 20-22 cm, through $119-737 \mathrm{~B}-6 \mathrm{H}-2,57-59 \mathrm{~cm}$, but most of this zone is removed at a hiatus present in the unrecovered interval between Cores 119737B-5H and 119-737B-6H. This hiatus spans at least the interval from 8.2 to $9.9 \mathrm{Ma}$ based on the near coincidence of the first occurrence of Thalassiosira burckliana (8.2 Ma in the topics) at the base of Core 119-737B-5H with the last common Denticulopsis dimorpha (9.9 Ma according to Gersonde and Burckle, 1990) in Sample 119-737B-6H-1, 16 cm (Barron, Larsen, et al., 1989). This hiatus is equivalent to widespread deep-sea hiatuses NH4 (in part) and NH5 of Keller and Barron (1987).

Samples 119-737B-6H-4, 57-59 cm, through 119-737B-8H-2, $57-59 \mathrm{~cm}$, are placed in NSOD Zone 10 based on the occurrence of Denticulopsis dimorpha below the first occurrence of Actinocyclus fryxellae (Table 6). The last occurrences of Denticulopsis praedimorpha and Nitzschia denticuloides (10.4 and $11.5 \mathrm{Ma}$, respectively, according to Gersonde and Burckle, 1990) are recognized in Sample 119-737B-7H-CC, coincident with the first occurrence of Rhizosolenia barboi (Table 6).

Based on the data presented in Table 6, NSOD Zone 9 is either missing or present in the interval between Samples 119-737B-8H$2,57-59 \mathrm{~cm}$, and 119-737B-8H-CC because of the coincidence of the first occurrences of $D$. dimorpha and $D$. praedimorpha in Sample 119-737B-8H-2, 57-59 cm. Examination of additional smear slides (shipboard data), however, revealed that Samples 119-737B-8H-2, $64 \mathrm{~cm}$, and 119-737B-8H-2, $100 \mathrm{~cm}$, contain $D$. praedimorpha without $D$. dimorpha and would be assigned to a compressed NSOD Zone 9 whereas Sample 119-737B-8H-3, 10 $\mathrm{cm}$, should be placed in underlying NSOD Zone 8 .

The presence of Nitzschia denticuloides without D. praedimorpha in Samples 119-737B-8H-3, $10 \mathrm{~cm}$, through 119-737B$10 \mathrm{H}-3,57-59 \mathrm{~cm}$, places this interval into NSOD Zone 8 (12.6-13.5 Ma). The last occurrence of Crucidenticula nicobarica in Sample 119-737B-8H-CC approximates the NSOD 9/NSOD 8 zonal boundary (Table 6 ).

The lowest interval containing well-preserved diatoms, Samples 119-737B-11H-1, $103 \mathrm{~cm}$, through 119-737B-10H-CC, seemingly should be assigned to NSOD Zone 6 (14.3-15.3 Ma) based on the presence of Nitzschia grossepunctata and Denticulopsis hyalina below the first occurrence of $D$. hustedtii. Coincidence of the first occurrence of $N$. denticuloides $(13.5 \mathrm{Ma})$, the first common occurrence of $D$. hustedtii $(14.0 \mathrm{Ma})$, and the first occurrence of Denticulopsis hustedtii (14.2 Ma; ages after Gersonde and Burckle, 1990) in Sample 119-737B-10H-3, 57-59 cm, suggests the possibility of a hiatus immediately above Sample 119-737A-10H-CC, which removes NSOD Zone 7. However, coincidence of the first occurrence of $N$. denticuloides with the first common occurrence of $D$. hustedtii also is found at Sites 744, 266, and 278 (Baldauf and Barron, herein), which argues against different ages for these two datums. Similarly, the absence of $D$. hustedtii below the first common occurrence of $D$. hustedtii may be due to ecological exclusion. If this is the case, Samples 119$737 \mathrm{~B}-11 \mathrm{H}-3,103 \mathrm{~cm}$, through 119-737B-10H-CC also might be correlative with NSOD Zone 7 rather than NSOD Zone 6. The presence of Actinocyclus ingens var. nodus in this interval is supportive of either assignment (Gersonde and Burckle, 1990; Table 5).

\section{Site 744}

Site 744 was cored in the southern Kerguelen Plateau region as a companion site to Site 738 (Table 1 and Fig. 1). A 176.1-mthick uppermost Eocene to Quaternary sequence was recovered from the three holes cored. Diatoms are present from the latest Quaternary NSOD Zone 21 to the early Oligocene Rhizosolenia oligocenica Zone. Diatom abundance and preservation vary, but in general diatoms are common and moderately preserved throughout the recovered sequence. The occurrence of selected and stratigraphically useful species in Cores 119-744A-1H through $119-744 \mathrm{~A}-16 \mathrm{H}$ and $119-744 \mathrm{~B}-1 \mathrm{H}$ through $119-744 \mathrm{~B}-9 \mathrm{H}$ is listed in Tables 7 and 8, respectively.

Shipboard (Barron, Larsen, et al., 1989) and shore-based (Keating, this volume) magnetic polarity logs are correlated with magnetic anomalies $3 \mathrm{~A}$ through 13 for the diatom-bearing Miocene and Oligocene section recovered from Holes 744A (Cores 119-744A-10H through 119-744A-17H) and 744B (Cores 119$744 \mathrm{~B}-4 \mathrm{H}$ through $119-744 \mathrm{~B}-10 \mathrm{H}$ ) in Figures 4 and 5. The ranges of stratigraphically useful diatoms in the Miocene and Oligocene interval are compared with published age estimates (see the subsequent discussion) and the sequence of normal and reversed polarity events in the Berggren et al. (1985) magnetic polarity time scale to identify the magnetic anomalies. Inasmuch as core 
Table 4. Occurrence of selected diatom species observed from Holes 736A and 736C.

\begin{tabular}{|c|c|c|c|c|c|c|c|c|c|c|c|c|c|c|c|c|c|c|c|c|c|c|c|c|c|c|c|c|c|c|c|c|c|c|c|c|c|c|c|}
\hline & 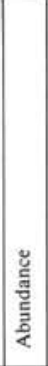 & 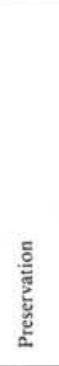 & 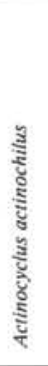 & 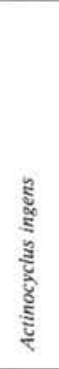 & 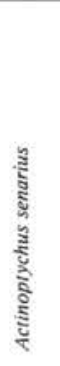 & 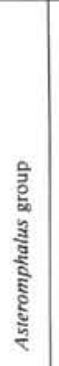 & 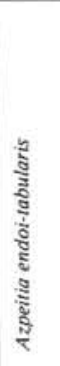 & 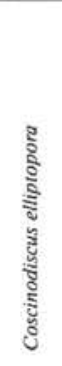 & 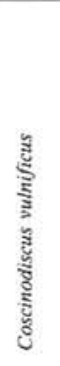 & 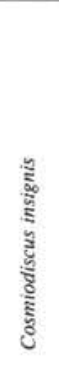 & $\begin{array}{l}\text { है } \\
\text { है } \\
\text { है } \\
\text { है } \\
\text { है } \\
\text { है }\end{array}$ & 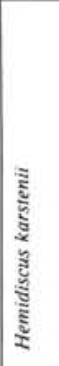 & 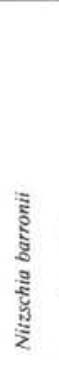 & 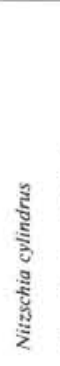 & 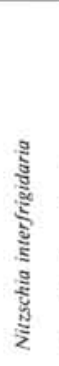 & 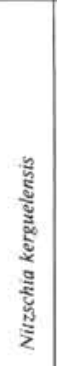 & 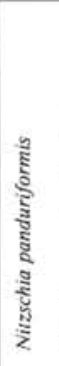 & 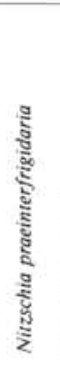 & 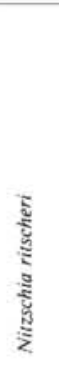 & 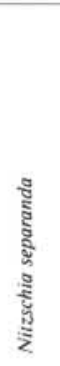 & 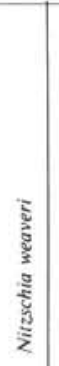 & 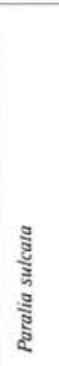 & 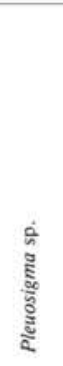 & 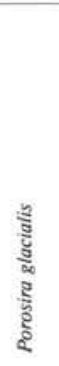 & 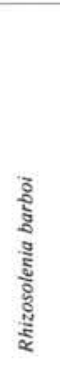 & 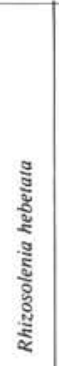 & 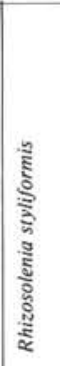 & 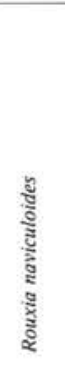 & 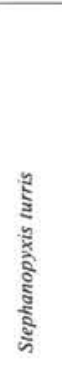 & 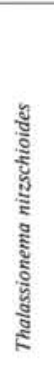 & 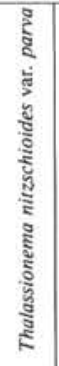 & 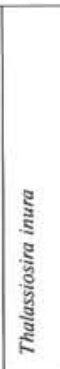 & 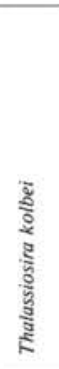 & 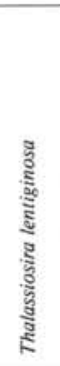 & 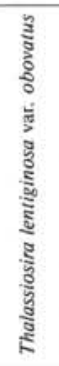 & 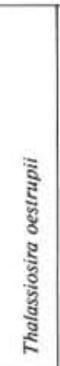 & 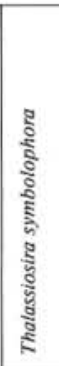 & 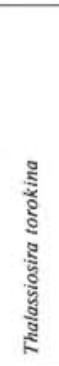 & 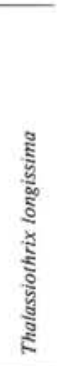 \\
\hline $736 \mathrm{~A}-$ & & & & & & & & & & & & & & & & & & & & & & & & & & & & & & & & & & & & & & & \\
\hline & $\begin{array}{l}\mathrm{A} \\
\mathrm{A}\end{array}$ & $\begin{array}{c}\mathrm{M} \\
\mathrm{M} \\
\mathrm{G} \\
\mathrm{G} \\
\mathrm{M}\end{array}$ & $\overline{\mathrm{F}}$ & - & $\begin{array}{l}\bar{z} \\
\bar{z} \\
\bar{z}\end{array}$ & $\begin{array}{l}\mathrm{R} \\
\mathrm{R} \\
\mathrm{R} \\
\mathrm{R}\end{array}$ & $\underline{\mathrm{R}}$ & $\begin{array}{l}\bar{z} \\
\bar{z} \\
\end{array}$ & $\begin{array}{l}\bar{z} \\
\bar{z} \\
\bar{z}\end{array}$ & - & $\begin{array}{l}\mathrm{F} \\
\mathrm{F} \\
\mathrm{C} \\
\mathrm{F} \\
\mathrm{F}\end{array}$ & - & $\mathrm{R}$ & $\bar{p}$ & - & & & $\begin{array}{l}\bar{z} \\
\bar{z} \\
\overline{-}\end{array}$ & $c$ & $\begin{array}{l}\bar{z} \\
\bar{E}\end{array}$ & - & $\begin{array}{l}R \\
R\end{array}$ & $\begin{array}{l}- \\
\bar{Z} \\
\bar{R}\end{array}$ & - & $\begin{array}{l}\bar{z} \\
\bar{z} \\
\end{array}$ & - & $R$ & $\begin{array}{l}z \\
z \\
z\end{array}$ & $\begin{array}{l}\bar{z} \\
\bar{z}\end{array}$ & $\bar{R}$ & $\begin{array}{l}z \\
z \\
z\end{array}$ & $\begin{array}{l}\mathrm{F} \\
\mathrm{R}\end{array}$ & $\begin{array}{l}z \\
z \\
z\end{array}$ & $F$ & $\begin{array}{l}\bar{z} \\
\bar{z}\end{array}$ & & $\begin{array}{l}\mathrm{R} \\
\mathrm{R} \\
\mathrm{F} \\
\mathrm{R} \\
\end{array}$ & - & $\begin{array}{l}\mathrm{F} \\
\mathrm{R} \\
\mathrm{F} \\
\mathrm{F} \\
\mathrm{F}\end{array}$ \\
\hline & & $\begin{array}{l}\mathrm{G} \\
\mathrm{M} \\
\mathrm{G}\end{array}$ & - & $\begin{array}{c}-\bar{r} \\
R \\
R \\
R \\
R\end{array}$ & - & $\mathbf{R}$ & $\overline{\bar{R}} \overline{\bar{R}}$ & $\begin{array}{l}\bar{z} \\
\bar{z}\end{array}$ & $\begin{array}{l}\bar{y} \\
\bar{z}\end{array}$ & - & $\mathrm{R}$ & & $\overline{\mathrm{R}}$ & & - & & & & C & $\mathbf{R}$ & - & - & - & $\bar{z}$ & $\bar{z}$ & - & & $\bar{z}$ & $\begin{array}{l}\bar{z} \\
\bar{z}\end{array}$ & $\bar{z}$ & $\begin{array}{l}z \\
z \\
z\end{array}$ & & $\begin{array}{l}\bar{z} \\
\bar{z} \\
z\end{array}$ & - & $\begin{array}{l}\bar{z} \\
\bar{z}\end{array}$ & & $\frac{R}{-}$ & - & $\stackrel{\mathrm{F}}{\mathrm{C}}$ \\
\hline & & M & $\frac{\mathrm{F}}{\mathrm{F}}$ & $\begin{array}{l}\mathrm{R} \\
\mathrm{R} \\
\mathrm{R} \\
\mathrm{R} \\
\mathrm{C}\end{array}$ & $\begin{array}{l}\frac{R}{R} \\
R \\
-\end{array}$ & $\overrightarrow{\mathrm{R}}$ & & $\bar{z}$ & $\bar{z}$ & - & $\overline{0}$ & & - & & - & & $R$ & - & $\mathrm{C}$ & $\overline{\bar{R}}$ & $2-1$ & $\overline{-}$ & - & - & & & & $\bar{z}$ & $=$ & $\bar{z}$ & $\begin{array}{l}\bar{z} \\
\overline{-}\end{array}$ & & $\begin{array}{l}\bar{z} \\
\bar{z} \\
\bar{z}\end{array}$ & & $\bar{z}$ & & $\begin{array}{l}\bar{z} \\
\bar{z} \\
\bar{z}\end{array}$ & $\bar{z}$ & $\begin{array}{l}R \\
R \\
R \\
- \\
-\end{array}$ \\
\hline & & $\begin{array}{l}\mathrm{G} \\
\mathrm{G} \\
\mathrm{M}\end{array}$ & - & $\mathrm{F}$ & - & - & $R$ & $\mathrm{~F}$ & $\overline{-}$ & - & $\begin{array}{l}F \\
R \\
\\
\bar{R}\end{array}$ & - & $F$ & & - & & - & & & - & -1 & $\overline{-}$ & - & $\bar{z}$ & & $=$ & $\pi$ & $\begin{array}{l}\bar{z} \\
\bar{z} \\
z\end{array}$ & $\begin{array}{l}\bar{z} \\
\bar{z} \\
=\end{array}$ & $R$ & $\begin{array}{l}F \\
= \\
= \\
-\end{array}$ & & $\begin{array}{l}\bar{z} \\
\bar{z} \\
\bar{z}\end{array}$ & & $\begin{array}{l}\bar{z} \\
\bar{z} \\
\bar{z}\end{array}$ & & $\begin{array}{l}\mathbf{R} \\
= \\
= \\
-\end{array}$ & $\overline{-}$ & $\begin{array}{l}\mathrm{F} \\
\mathrm{F} \\
\mathrm{F}\end{array}$ \\
\hline & & $\begin{array}{l}\mathrm{G} \\
\mathrm{M}\end{array}$ & $\overline{\mathrm{R}}$ & $\begin{array}{l}F \\
R\end{array}$ & $\bar{z}$ & $\overline{-}$ & $\begin{array}{l}- \\
\bar{z} \\
\overline{-} \\
-\end{array}$ & $\frac{\hat{F}}{R}$ & $\begin{array}{l}\bar{z} \\
\bar{z}\end{array}$ & $\bar{z}$ & $\begin{array}{l}\bar{R} \\
R \\
\bar{R}\end{array}$ & - & R & $\Xi$ & - & $R$ & - & $\bar{z}$ & $\bar{z}$ & $\begin{array}{l}\bar{z} \\
\bar{z} \\
\overline{-}\end{array}$ & -1 & R & - & $\overline{-}$ & $\begin{array}{l}\frac{\mathrm{I}}{-} \\
\overline{\mathrm{R}}\end{array}$ & - & & - & $\bar{z}$ & $\stackrel{F}{-}$ & - & - & $\bar{z}$ & $\stackrel{R}{-}$ & $\begin{array}{l}\bar{z} \\
\bar{z}\end{array}$ & & $\begin{array}{l}\bar{z} \\
\bar{z} \\
-\end{array}$ & - & $\begin{array}{l}\mathrm{F} \\
\mathrm{R}\end{array}$ \\
\hline & 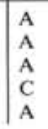 & \begin{tabular}{|l} 
M \\
G \\
M \\
G
\end{tabular} & $\begin{array}{l}\bar{F} \\
\overline{-} \\
-\end{array}$ & $\begin{array}{l}\text { C } \\
\text { F } \\
\text { A }\end{array}$ & $\begin{array}{l}\bar{z} \\
\bar{z}\end{array}$ & $\underline{\mathbf{R}}$ & $\begin{array}{l}\bar{z} \\
\bar{z} \\
\bar{z}\end{array}$ & $\frac{\mathrm{F}}{\mathrm{F}}$ & $\begin{array}{l}\bar{y} \\
\bar{z}\end{array}$ & $\begin{array}{l}\bar{z} \\
\bar{z}\end{array}$ & $\begin{array}{l}\bar{z} \\
= \\
=\end{array}$ & $\bar{z}$ & $\mathbf{R}$ & $\begin{array}{l}\bar{z} \\
\bar{z}\end{array}$ & $\bar{z}$ & $R$ & $\begin{array}{l}\bar{z} \\
\bar{R}\end{array}$ & $\begin{array}{l}\bar{z} \\
\bar{z}\end{array}$ & $\frac{R}{R}$ & $\begin{array}{l}\bar{z} \\
\bar{z}\end{array}$ & \pm & $\bar{z}$ & $\underline{-}$ & $\bar{z}$ & $\frac{\frac{R}{R}}{F}$ & $\underline{F}$ & |R & - & $\bar{z}$ & $\mathbf{R}$ & $\mathbf{R}$ & R & - & F & $\begin{array}{l}\overline{-} \\
\overline{-}\end{array}$ & $\mathbf{F}$ & $\frac{\mathrm{R}^{\mathrm{R}}}{-}$ & & \\
\hline
\end{tabular}


Table 4 (Continued).

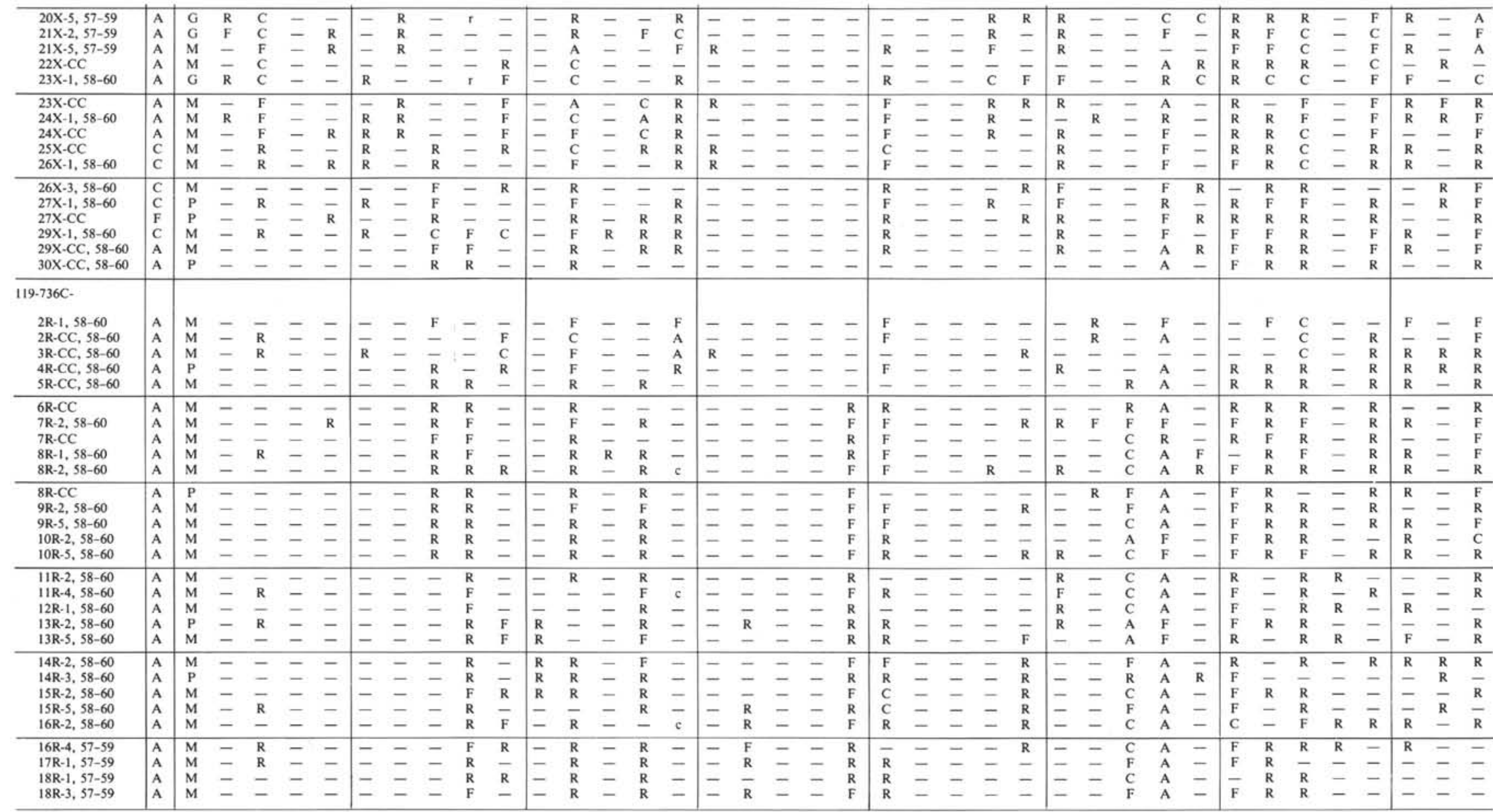

Note: $\mathrm{Abundance}: \mathrm{A}=$ abundant, $\mathrm{C}=$ common, $\mathrm{F}=$ few, $\mathrm{R}=$ rare; preservation: $\mathrm{G}=$ good, $\mathrm{M}=$ moderate, $\mathrm{P}=$ poor; lowercase letters indicate reworking. 
Table 5. Occurrence of selected diatom species observed from Hole 737A.

\begin{tabular}{|c|c|c|c|c|c|c|c|c|c|c|c|c|c|c|c|c|c|c|c|c|c|c|c|c|}
\hline $\begin{array}{l}\text { Core, section, } \\
\text { interval (cm) }\end{array}$ & 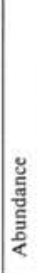 & 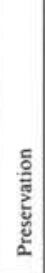 & 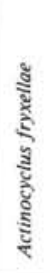 & 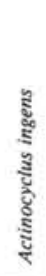 & 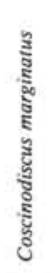 & 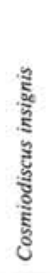 & 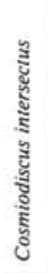 & 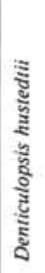 & 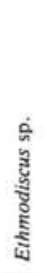 & 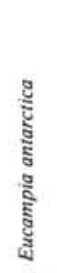 & 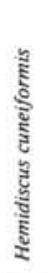 & 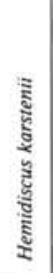 & 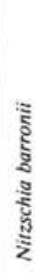 & 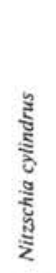 & 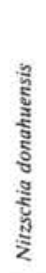 & 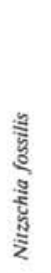 & $\begin{array}{l}\text { है } \\
\text { है } \\
\text { है } \\
\text { है } \\
\text { है } \\
\text { है }\end{array}$ & 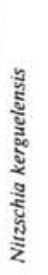 & $\begin{array}{l}\text { हूँ } \\
\text { है } \\
\text { है } \\
\text { है } \\
\text { है }\end{array}$ & 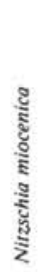 & $\begin{array}{l}\text { है } \\
\text { है } \\
\text { है } \\
\text { है } \\
\text { है } \\
\text { है }\end{array}$ & 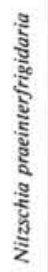 & 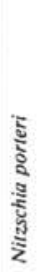 & 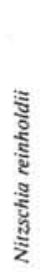 \\
\hline \multicolumn{25}{|l|}{$119.737 \mathrm{~A}}$. \\
\hline $1 \mathrm{H}-1,57-59$ & A & $\mathrm{G}$ & - & - & - & - & - & - & - & C & - & - & F & - & - & - & - & A & - & - & $R$ & - & - & - \\
\hline $1 \mathrm{H}-2,57-59$ & $\hat{A}$ & $\mathrm{M}$ & - & - & - & - & - & - & $\mathrm{R}$ & - & - & - & $\mathrm{F}$ & - & - & - & $R$ & $\mathrm{c}$ & - & - & $\hat{R}$ & $\mathrm{~F}$ & - & - \\
\hline IH-CC & A & M & - & - & - & - & - & - & - & - & - & - & c & - & - & - & $\mathrm{F}$ & - & - & - & - & $F$ & - & - \\
\hline $2 \mathrm{H}-2,57-59$ & A & M & - & - & - & - & - & - & - & - & - & - & $\mathrm{F}$ & - & - & - & $\mathrm{F}$ & - & - & - & - & $R$ & - & - \\
\hline $2 \mathrm{H}-5,57-59$ & & & - & - & - & - & - & & & & & - & c & - & - & - & C & - & - & - & - & $\mathrm{R}$ & - & - \\
\hline $2 \mathrm{H}-\mathrm{CC}$ & A & $\mathrm{M}$ & - & - & - & - & - & - & - & - & - & - & $F$ & - & - & - & $F$ & - & - & - & - & $F$ & - & - \\
\hline $3 \mathrm{H} \cdot 2,57$ & A & $\mathrm{G}$ & - & - & - & - & - & - & - & - & - & - & F & - & - & - & $\mathrm{F}$ & - & - & - & - & $\mathrm{R}$ & - & - \\
\hline $3 \mathrm{H}-5,57-59$ & A & $\mathrm{G}$ & - & - & $\mathrm{R}$ & $\mathbf{R}$ & - & - & - & - & - & - & C & - & - & - & $R$ & - & - & - & - & C & - & - \\
\hline $4 \mathrm{H}-2,57-59$ & A & G & - & - & - & - & - & - & - & - & - & - & $\mathrm{F}$ & - & - & - & $R$ & - & - & - & - & $\mathrm{F}$ & - & - \\
\hline $4 \mathrm{H}-5,57-59$ & A & G & - & - & - & - & - & - & - & - & - & - & $\mathrm{F}$ & - & - & - & $\mathbf{R}$ & - & - & - & - & $\mathrm{F}$ & - & - \\
\hline $4 \mathrm{H} \cdot \mathrm{CC}$ & A & $\mathrm{G}$ & - & - & $\mathrm{R}$ & - & - & - & - & $R$ & - & - & $\mathrm{F}$ & - & - & - & $R$ & - & - & - & - & $\mathrm{R}$ & - & - \\
\hline 51 & A & $\mathrm{G}$ & - & - & - & - & - & - & $\pi$ & - & - & $\mathrm{R}$ & F & - & - & - & $\mathrm{R}$ & - & $\mathbf{R}$ & - & - & $\mathrm{R}$ & - & - \\
\hline $5 \mathrm{H}-5$, & A & M & - & $\mathrm{R}$ & - & - & - & - & - & F & - & $\cdots$ & R & - & - & - & $\mathrm{F}$ & - & - & - & - & - & - & - \\
\hline $6 \mathrm{H}-1,57-59$ & A & M & - & - & - & - & - & - & - & C & - & - & $\mathbf{R}$ & - & - & - & $F$ & - & - & - & - & $\mathbf{R}$ & - & $\mathbf{R}$ \\
\hline $6 \mathrm{H}-5,57-59$ & C & M & - & - & - & - & - & - & - & $\mathrm{F}$ & - & - & R & - & - & - & - & - & - & - & - & $\mathrm{F}$ & - & $\mathbf{R}$ \\
\hline $6 \mathrm{H} \cdot \mathrm{CC}$ & A & M & - & - & - & $R$ & - & - & - & $F$ & - & - & $\mathrm{R}$ & - & - & - & - & - & - & - & - & $\mathrm{R}$ & - & - \\
\hline $7 \mathrm{H}-2,57-59$ & $\ddot{A}_{\mathrm{A}}$ & $\mathrm{G}$ & - & - & - & R & - & - & - & - & - & - & c & $\mathrm{R}$ & - & - & - & - & - & - & - & $\mathrm{F}$ & & - \\
\hline $7 \mathrm{H}-5,57-59$ & A & M & - & - & R & - & - & - & - & - & - & - & $\mathrm{F}$ & - & - & - & - & - & - & - & - & $\mathbf{R}$ & - & - \\
\hline $7 \mathrm{H}-\mathrm{C}$ & A & M & - & - & $\mathbf{R}$ & - & - & - & - & - & - & - & $\mathrm{F}$ & - & - & - & - & - & - & - & - & $\mathrm{R}$ & - & - \\
\hline $8 \mathrm{H}-3,57-59$ & A & M & - & - & - & - & - & - & $\mathrm{R}$ & - & - & $\mathrm{F}$ & - & $\mathrm{R}$ & - & - & - & - & - & - & - & $\mathrm{R}$ & - & $\mathrm{F}$ \\
\hline $8 \mathrm{H}-6,57-59$ & A & $\mathrm{G}$ & - & - & - & - & - & - & $\mathrm{R}$ & - & - & - & - & $R$ & - & R & - & - & - & - & - & $\mathrm{R}$ & - & $\mathrm{F}$ \\
\hline $8 \mathrm{H}-\mathrm{CC}$ & A & M & - & - & R & $R$ & - & - & - & - & - & $\mathrm{R}$ & - & - & - & - & - & - & - & - & - & $R$ & - & $\mathrm{F}$ \\
\hline $9 \mathrm{H}-2,57-59$ & A & $\mathrm{G}$ & - & - & - & R & - & - & - & - & - & $R$ & - & $\pi$ & - & - & - & - & - & - & $\overline{-}$ & $R$ & - & $\mathrm{F}$ \\
\hline $9 \mathrm{H}-5,57-59$ & A & M & - & - & $\mathbf{R}$ & - & - & - & - & - & - & R & - & $\mathrm{F}$ & - & $\mathbf{R}$ & - & - & - & - & $\mathrm{R}$ & $\mathrm{R}$ & - & $\mathrm{R}$ \\
\hline $9 \mathrm{H}-\mathrm{CC}$ & A & M & - & - & - & - & - & - & - & - & - & $\vec{F}$ & - & - & - & $\mathrm{R}$ & - & - & - & - & - & - & - & $\mathrm{F}$ \\
\hline $10 \mathrm{H}-4,57-59$ & c & $\mathrm{M}$ & - & - & - & $R$ & - & - & - & - & - & $\mathrm{F}$ & - & - & - & $R$ & - & - & - & - & - & - & - & $F$ \\
\hline & L & $\mathrm{P}$ & - & - & - & - & - & - & - & 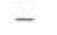 & $\mathrm{F}$ & R & & & - & $\ddot{R}$ & 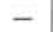 & & $\mathrm{K}$ & - & - & & & $\mathrm{R}$ \\
\hline $10 \mathrm{H}-6$ & C & $\mathrm{P}$ & - & - & $\mathrm{F}$ & R & - & - & c & - & $\mathrm{R}$ & $\hat{R}$ & - & R & - & $\ddot{-}$ & - & . & $\hat{R}$ & - & - & - & - & $\mathrm{F}$ \\
\hline $10 \mathrm{H}-\mathrm{C}$ & A & $\mathrm{P}$ & - & - & $\mathrm{F}$ & R & - & - & A & $\mathbf{R}$ & $\underline{-}$ & $\hat{R}$ & - & $\hat{R}$ & - & - & - & - & - & - & - & - & - & $\mathrm{R}$ \\
\hline $11 \mathrm{H}-2,57-59$ & A & M & - & - & $\mathrm{R}$ & - & $R$ & - & A & - & - & $\mathrm{F}$ & - & R & - & $\mathbf{R}$ & - & - & & - & - & - & - & $\mathbf{R}$ \\
\hline $11 \mathrm{H}-5,57-59$ & A & $\mathrm{G}$ & - & - & $\mathrm{R}$ & R & - & - & A & - & - & $\mathrm{C}$ & - & $\mathrm{R}$ & - & $\mathrm{R}$ & - & - & - & $\mathrm{R}$ & - & - & - & C \\
\hline & $c$ & $\mathrm{P}$ & - & - & - & & 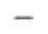 & & $c$ & - & - & $\mathrm{F}$ & & - & - & - & - & . & . & $\mathrm{R}$ & - & & - & $F$ \\
\hline $57-59$ & c & $\mathrm{G}$ & - & - & $\mathrm{F}$ & - & - & - & A & - & - & $\mathrm{F}$ & - & - & - & R & - & - & - & - & - & . & - & $\mathrm{F}$ \\
\hline $57-59$ & A & $\mathrm{G}$ & - & - & $\mathrm{F}$ & - & - & - & A & - & - & $\mathrm{F}$ & - & - & - & $\hat{R}$ & - & - & - & $R$ & - & - & - & $\mathrm{F}$ \\
\hline $12 \mathrm{H} \cdot \mathrm{CC}$ & A & M & - & - & $\mathbf{R}$ & - & - & - & A & - & - & R & - & - & - & $\mathrm{R}$ & - & - & - & $\mathrm{K}$ & - & - & - & $\mathrm{R}$ \\
\hline $13 \mathrm{H}-2,57-59$ & A & $\mathrm{M}$ & - & - & - & - & - & - & A & - & - & R & - & - & - & $P$ & - & - & - & $\mathbf{R}$ & - & - & - & $F$ \\
\hline & A & N & - & - & R & - & - & - & $c$ & - & - & R & & - & - & $\mathrm{R}$ & - & - & $\mathrm{R}$ & - & 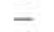 & - & & . \\
\hline $15 \mathrm{H}-5,57-59$ & A & G & - & - & R & - & - & - & $\bar{x}$ & - & $\mathbf{R}$ & $\mathbf{R}$ & - & - & - & $R$ & - & - & - & - & - & - & $R$ & $\mathbf{R}$ \\
\hline $15 \mathrm{H}-7$ & A & $\mathrm{G}$ & - & - & R & - & - & - & $F$ & - & - & $\mathrm{F}$ & - & - & - & $\mathbf{R}$ & - & - & - & - & - & - & - & $\mathrm{R}$ \\
\hline $15 \mathrm{H} \cdot \mathrm{CC}$ & A & $\mathrm{M}$ & - & - & - & - & - & - & - & - & $\mathrm{R}$ & - & - & $\mathrm{R}$ & - & R & - & - & - & $\mathrm{R}$ & - & - & - & $\mathbf{R}$ \\
\hline $16 \mathrm{H}-2,57-59$ & A & $\mathrm{G}$ & - & - & - & - & - & $\pi$ & $C$ & 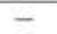 & $\mathrm{R}$ & $\mathrm{F}$ & - & $R$ & - & $\mathrm{F}$ & - & - & $=$ & - & - & - & - & $\mathbf{R}$ \\
\hline & 10 & s & - & 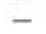 & $\mathbf{R}$ & & - & - & c & - & n & $\mathrm{F}$ & & 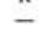 & & in & - & & & - & & & & D \\
\hline & A & $\mathrm{P}$ & - & - & - & - & - & 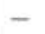 & $\mathrm{F}$ & - & - & - & - & - & 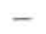 & - & - & - & - & - & & - & 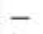 & - \\
\hline 17 & A & G & - & - & $\mathbf{R}$ & - & 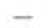 & 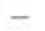 & A & - & - & $\mathbf{R}$ & & $p$ & - & $F$ & & 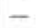 & - & - & & - & - & $\mathbf{R}$ \\
\hline $17 \mathrm{H}-5.57-59$ & A & $\mathrm{G}$ & - & - & R & - & $\mathrm{R}$ & - & A & - & - & - & - & $\mathbf{R}$ & - & $\mathrm{R}$ & - & - & - & $R$ & - & - & - & $\ddot{R}$ \\
\hline $17 \mathrm{H} \cdot \mathrm{CC}$ & A & $\mathrm{M}$ & - & - & $\mathbf{R}$ & - & - & $\mathbf{R}$ & C & - & $\mathrm{R}$ & $\mathrm{R}$ & - & - & - & $\mathrm{R}$ & - & - & - & - & - & - & - & $\mathbf{R}$ \\
\hline 18 & A & 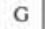 & 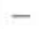 & 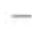 & - & $-\pi$ & R & - & A & & - & $\mathbf{R}$ & & - & & $\mathrm{F}$ & 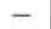 & - & - & $F$ & 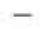 & - & - & - \\
\hline 18 & A & c & - & - & R & R & 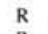 & - & A & - & - & - & & - & - & R & 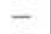 & - & - & $\mathrm{R}$ & - & - & - & R \\
\hline & A & $\Delta$ & - & - & - & - & $\mathbf{R}$ & - & c & & - & - & - & - & - & $\mathrm{R}$ & - & - & - & F & - & - & - & 2 \\
\hline $19 \mathrm{X}-1,57-59$ & A & $\mathrm{G}$ & - & - & $\mathbf{R}$ & $\mathbf{R}$ & $\mathrm{F}$ & - & A & - & - & $\mathbf{R}$ & - & $\mathbf{R}$ & - & $\hat{R}$ & - & - & $\mathrm{R}$ & $\mathrm{R}$ & - & - & - & $\mathbf{R}$ \\
\hline 19X-CC & A & $\mathrm{G}$ & - & - & - & - & - & - & A & - & - & - & 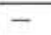 & - & - & $I$ & & & & & - & - & & - \\
\hline & & $P$ & - & - & - & - & 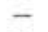 & - & A & & - & & & - & - & & -1 & - & 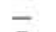 & & & - & $\mathrm{F}$ & - \\
\hline 21 & A & G & - & - & R & - & - & $\mathrm{R}$ & $\mathrm{F}$ & - & - & $\mathrm{R}$ & - & F & - & $\mathrm{R}$ & - & - & $\mathrm{F}$ & $\mathrm{R}$ & - & - & - & \\
\hline $57-59$ & A & G & $\mathrm{F}$ & - & - & - & $\mathrm{F}$ & - & - & - & $\mathbf{R}$ & $\mathrm{F}$ & - & $R$ & - & 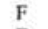 & - & - & - & - & 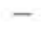 & - & F & $\mathbf{R}$ \\
\hline $23 \mathrm{X} \cdot \mathrm{CC}$ & A & $\mathrm{P}$ & - & - & R & - & $\mathrm{R}$ & - & - & - & - & $\mathbf{R}$ & - & $\mathrm{R}$ & - & $\mathrm{F}$ & - & - & - & - & - & - & $\mathrm{R}$ & - \\
\hline $25 X-2,57-59$ & A & $P$ & $\mathrm{R}$ & $R$ & $R$ & - & $F$ & - & -1 & - & $\mathrm{R}$ & - & - & $\mathrm{R}$ & - & $F$ & - & - & - & - & - & - & - & $\mathbf{R}$ \\
\hline & & M & $\mathrm{R}$ & $\mathrm{R}$ & R & & & 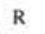 & & & $\mathbf{R}$ & & & - & & & & & & & & & - & - \\
\hline 25 & A & $\mathrm{G}$ & $\hat{R}$ & $\hat{\mathrm{F}}$ & $\underline{n}$ & - & $\mathrm{F}$ & - & - & 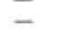 & $\underline{-}$ & $\bar{R}$ & - & 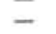 & 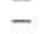 & F & - & 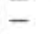 & 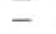 & - & & - & - & - \\
\hline $57-59$ & A & G & $\mathrm{F}$ & - & - & - & F & $\mathrm{R}$ & - & - & R & - & - & - & $\mathrm{R}$ & 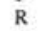 & - & - & - & - & - & - & - & - \\
\hline $26 \mathrm{X}-\mathrm{CC}$ & A & $\mathrm{M}$ & $\mathrm{F}$ & $R$ & - & - & $\mathrm{F}$ & c & - & - & $\ddot{-}$ & - & - & C & $\ddot{R}$ & - & - & - & $\mathrm{F}$ & - & - & - & - & - \\
\hline $27 X-1,57-5$ & A & G & $\mathrm{F}$ & $\mathrm{F}$ & $\mathbf{R}$ & - & $F$ & A & - & - & - & - & - & A & - & $\mathrm{R}$ & - & - & $\mathrm{F}$ & - & - & - & -1 & $F$ \\
\hline & A & M & $\mathrm{F}$ & $\mathbf{R}$ & R & - & $\mathrm{F}$ & $c$ & & & 8 & $\mathbf{R}$ & & $\mathrm{F}$ & $P$ & & -1 & - & F & 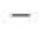 & & -1 & 1, & $R$ \\
\hline 27 & A & M & - & - & $\mathrm{F}$ & - & - & C & 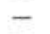 & 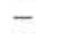 & - & - & - & - & 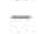 & - & -1 & 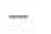 & - & - & 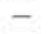 & - & - & - \\
\hline $28 \times-1$ & A & $P$ & - & $\mathrm{F}$ & $\mathbf{R}$ & - & - & A & - & - & R & - & - & - & - & - & - & - & - & - & - & - & - & - \\
\hline $28 \mathrm{X}-\mathrm{CC}$ & & M & - & R & F & - & - & - & - & 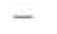 & - & - & - & - & $\mathrm{R}$ & - & & - & - & - & - & - & - & - \\
\hline
\end{tabular}

Note: Abundance: $\mathrm{A}=$ abundant, $\mathrm{C}=$ common, $\mathrm{F}=\mathrm{few}, \mathrm{R}=$ rare; preservation: $\mathrm{G}=$ good, $\mathrm{M}=$ moderate, $\mathrm{P}=$ poor; lowercase letters indicate reworking. 
Table 5 (Continued).

\begin{tabular}{|c|c|c|c|c|c|c|c|c|c|c|c|c|c|c|c|c|c|c|c|c|c|c|c|c|c|c|c|}
\hline & $\begin{array}{l}\text { हूँ } \\
\text { है } \\
\text { हैँ } \\
\text { है }\end{array}$ & 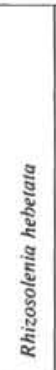 & 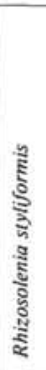 & 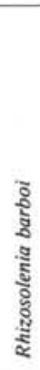 & 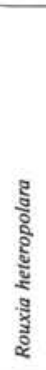 & 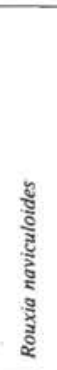 & 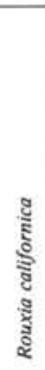 & 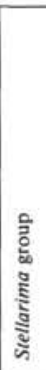 & 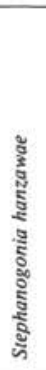 & 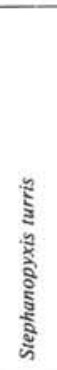 & 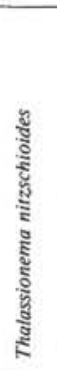 & 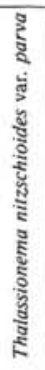 & 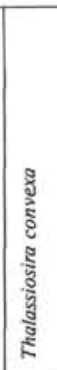 & 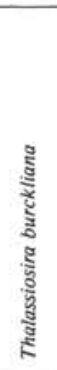 & 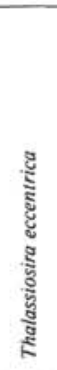 & $\begin{array}{l}\text { हूँ } \\
\text { हूँ } \\
\text { है } \\
\frac{\text { gू }}{\text { है }}\end{array}$ & 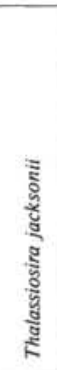 & 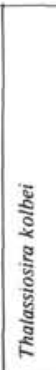 & 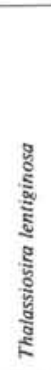 & $\begin{array}{l}\text { gू } \\
\text { हूँ } \\
\text { हू } \\
\text { gू } \\
\text { gू } \\
\text { gू }\end{array}$ & 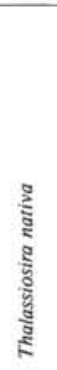 & 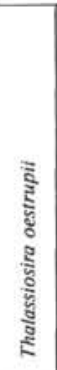 & 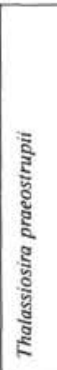 & 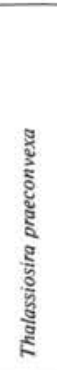 & 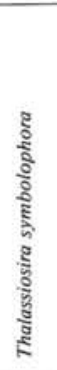 & 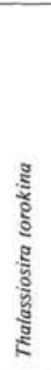 & $\begin{array}{l}\frac{\pi}{5} \\
\frac{\text { s. }}{5} \\
\frac{5}{5} \\
\frac{5}{5}\end{array}$ \\
\hline$\overline{\mathrm{F}}$ & $\begin{array}{l}\text { F } \\
\text { C } \\
\text { F } \\
\text { C } \\
\text { C }\end{array}$ & $\begin{array}{l}\bar{z} \\
\bar{z}\end{array}$ & $\begin{array}{l}\bar{R} \\
R \\
F \\
R\end{array}$ & $\begin{array}{l}\bar{z} \\
\overline{-}\end{array}$ & $\begin{array}{l}\bar{z} \\
\bar{z}\end{array}$ & $\begin{array}{l}\overline{-} \\
\bar{R} \\
\mathrm{~F} \\
-\end{array}$ & $\begin{array}{l}\bar{z} \\
\bar{z}\end{array}$ & $\begin{array}{l}\bar{z} \\
\bar{z}\end{array}$ & $\begin{array}{l}\bar{z} \\
\bar{z}\end{array}$ & $\frac{\mathrm{R}}{\frac{\mathrm{R}}{\mathrm{R}}}$ & $\begin{array}{l}\mathrm{F} \\
\mathrm{C} \\
\mathrm{C} \\
\mathrm{C} \\
\mathrm{F}\end{array}$ & $\begin{array}{l}\bar{z} \\
\bar{z} \\
\bar{z}\end{array}$ & $\begin{array}{l}\bar{z} \\
\bar{z} \\
\bar{y}\end{array}$ & $\begin{array}{l}\bar{z} \\
\bar{z}\end{array}$ & $\begin{array}{l}\mathrm{R} \\
\frac{\bar{R}}{\mathrm{R}} \\
\mathrm{R} \\
\mathrm{R}\end{array}$ & $\begin{array}{l}R \\
R \\
R \\
\bar{F}\end{array}$ & $\begin{array}{l}- \\
\bar{R} \\
R \\
R\end{array}$ & $\begin{array}{l}\overline{-} \\
\bar{z} \\
\bar{F}\end{array}$ & $\begin{array}{l}\mathrm{C} \\
\overline{-} \\
\bar{R} \\
\mathrm{R}\end{array}$ & $\begin{array}{l}\bar{z} \\
\bar{z}\end{array}$ & $\begin{array}{l}\bar{z} \\
\bar{I}\end{array}$ & $\begin{array}{l}\text { F } \\
C \\
F \\
F \\
F\end{array}$ & $\begin{array}{l}\bar{z} \\
\bar{z} \\
z\end{array}$ & $\begin{array}{l}\bar{z} \\
\bar{z}\end{array}$ & $\begin{array}{l}R \\
R \\
R \\
R \\
R \\
R\end{array}$ & $\frac{\bar{R}}{\frac{\bar{R}}{\mathrm{~F}}}$ & $\mathrm{~A}$ \\
\hline $\begin{array}{l}R \\
\text { A } \\
\end{array}$ & $\begin{array}{l}\mathrm{F} \\
\mathrm{C} \\
\mathrm{F} \\
\mathrm{C} \\
\mathrm{F}\end{array}$ & $\begin{array}{l}\bar{z} \\
\bar{I} \\
=\end{array}$ & $\begin{array}{l}F \\
R \\
R \\
R \\
F \\
R\end{array}$ & $\begin{array}{l}\bar{I} \\
\overline{\mathrm{R}} \\
\overline{\mathrm{R}}\end{array}$ & $\begin{array}{l}\bar{z} \\
\bar{z}\end{array}$ & $\begin{array}{l}R \\
R \\
F \\
- \\
-\end{array}$ & $\begin{array}{l}\bar{z} \\
\bar{z}\end{array}$ & $\begin{array}{l}\bar{z} \\
\bar{z} \\
=\end{array}$ & $\begin{array}{l}\bar{z} \\
\bar{z}\end{array}$ & $\begin{array}{l}\overline{-} \\
\overline{\mathrm{R}} \\
\underline{-}\end{array}$ & $\begin{array}{l}\mathrm{F} \\
\bar{F} \\
\mathrm{R} \\
\mathrm{F}\end{array}$ & $\begin{array}{l}\bar{z} \\
\bar{z} \\
\bar{z}\end{array}$ & $\begin{array}{l}\bar{z} \\
\bar{z} \\
z\end{array}$ & $\begin{array}{l}\bar{I} \\
\bar{I}\end{array}$ & $\begin{array}{l}\mathrm{F} \\
\mathrm{F} \\
\mathrm{R} \\
\mathrm{F}\end{array}$ & $\begin{array}{l}\mathrm{F} \\
\mathrm{F} \\
\mathrm{F} \\
\mathrm{F} \\
\mathrm{F}\end{array}$ & $\begin{array}{l}\mathbf{R} \\
\mathbf{R} \\
\mathbf{R} \\
\mathbf{R} \\
\mathbf{R}\end{array}$ & $\begin{array}{l}\overline{-} \\
= \\
-\end{array}$ & $\begin{array}{l}\frac{R}{R} \\
\frac{R}{-}\end{array}$ & $\begin{array}{l}\bar{z} \\
\bar{z} \\
\bar{y}\end{array}$ & $\begin{array}{l}\bar{I} \\
\bar{I}\end{array}$ & $\begin{array}{l}\mathrm{F} \\
\bar{F} \\
F \\
R\end{array}$ & $\begin{array}{l}\bar{z} \\
\bar{z} \\
=\end{array}$ & $\begin{array}{l}\bar{z} \\
\bar{z}\end{array}$ & $\begin{array}{l}R \\
R \\
R \\
R \\
R\end{array}$ & $\begin{array}{l}\overline{-} \\
\bar{R} \\
\mathrm{R} \\
\mathrm{F}\end{array}$ & $\begin{array}{l}\text { A } \\
\text { A } \\
\text { A }\end{array}$ \\
\hline $\bar{z}$ & $\begin{array}{l}\mathrm{C} \\
\mathrm{F} \\
\mathrm{F} \\
\mathrm{F} \\
\mathrm{R}\end{array}$ & $\begin{array}{l}\mathrm{R} \\
\mathbf{Z} \\
\mathbf{I}\end{array}$ & $\begin{array}{l}\mathrm{F} \\
\mathrm{R} \\
\mathrm{R} \\
\mathrm{F} \\
\mathrm{R}\end{array}$ & $\begin{array}{l}\bar{z} \\
\overline{-}\end{array}$ & $\begin{array}{l}\bar{z} \\
\bar{z}\end{array}$ & $\begin{array}{l}\bar{R} \\
\bar{R} \\
F\end{array}$ & $\begin{array}{l}\bar{z} \\
\bar{z}\end{array}$ & $\begin{array}{l}\overline{-} \\
\bar{z} \\
\bar{R}\end{array}$ & $\begin{array}{l}\bar{R} \\
\overline{-} \\
-\end{array}$ & $\begin{array}{l}\mathrm{R} \\
\mathrm{R} \\
\mathrm{R} \\
\mathrm{F} \\
\mathrm{R}\end{array}$ & $\begin{array}{l}\mathrm{F} \\
\mathrm{R} \\
\mathrm{F} \\
\mathrm{F} \\
\mathrm{F}\end{array}$ & $\begin{array}{l}\bar{z} \\
\bar{z} \\
\bar{z}\end{array}$ & $\begin{array}{l}\overline{\bar{R}} \\
\overline{\mathrm{R}} \\
\overline{-}\end{array}$ & $\begin{array}{l}\bar{I} \\
\bar{I} \\
\end{array}$ & $\begin{array}{l}F \\
F \\
F \\
F \\
R\end{array}$ & $\begin{array}{l}F \\
F \\
R \\
F \\
F\end{array}$ & $\begin{array}{l}\mathbf{R} \\
\mathbf{R} \\
\mathbf{R} \\
\overline{\mathrm{F}}\end{array}$ & $\begin{array}{l}\overline{-} \\
\bar{z} \\
-\end{array}$ & $\begin{array}{l}\overline{\bar{R}} \\
\overline{\mathrm{R}}\end{array}$ & $\begin{array}{l}\bar{z} \\
\bar{z} \\
\bar{y}\end{array}$ & $\begin{array}{l}\bar{I} \\
\bar{I} \\
\bar{y}\end{array}$ & $\begin{array}{l}R \\
F \\
F \\
F \\
F\end{array}$ & $\bar{z}$ & $\begin{array}{l}\bar{z} \\
\bar{z}\end{array}$ & $\begin{array}{l}\bar{R} \\
\bar{R} \\
F\end{array}$ & $\begin{array}{l}\mathrm{F} \\
\mathrm{F} \\
\mathrm{C} \\
\mathrm{R} \\
\mathrm{R}\end{array}$ & $\mathrm{F}$ \\
\hline $\begin{array}{l}\bar{z} \\
\bar{z}\end{array}$ & $\begin{array}{l}\bar{F} \\
R \\
R \\
R\end{array}$ & $\begin{array}{l}= \\
= \\
=\end{array}$ & $\begin{array}{l}R \\
R \\
R \\
R \\
-\end{array}$ & $\begin{array}{l}\bar{R} \\
R \\
R \\
F\end{array}$ & $\begin{array}{l}\bar{z} \\
\bar{z}\end{array}$ & $\begin{array}{l}\bar{R} \\
R \\
R \\
R \\
R\end{array}$ & $\begin{array}{l}\mathrm{R} \\
\overline{-} \\
\overline{-}\end{array}$ & $\begin{array}{l}\mathrm{R} \\
\frac{\mathrm{Z}}{\mathrm{R}}\end{array}$ & $\begin{array}{l}\bar{z} \\
\bar{z} \\
\bar{R}\end{array}$ & $\begin{array}{l}\bar{C} \\
\mathrm{C} \\
\mathrm{F} \\
\mathrm{F}\end{array}$ & $\begin{array}{l}\mathrm{R} \\
\mathrm{A} \\
\mathrm{A} \\
\mathrm{A} \\
\mathrm{A}\end{array}$ & $\begin{array}{l}- \\
\overline{-} \\
\bar{R} \\
\mathrm{~F}\end{array}$ & $\bar{z}$ & $\bar{z}$ & $\begin{array}{l}\mathrm{F} \\
\mathrm{F} \\
\mathrm{R} \\
\mathrm{R} \\
-\end{array}$ & $\begin{array}{l}\mathrm{F} \\
\mathrm{C} \\
\mathrm{F} \\
\mathrm{F} \\
\mathrm{R}\end{array}$ & $\begin{array}{l}\mathrm{R} \\
\mathrm{R} \\
\mathrm{R} \\
\mathrm{R} \\
-\end{array}$ & $\begin{array}{l}\bar{z} \\
\bar{I} \\
\bar{z}\end{array}$ & $\begin{array}{l}\bar{z} \\
\bar{z}\end{array}$ & $\begin{array}{l}\bar{z} \\
\bar{z}\end{array}$ & $\begin{array}{l}\bar{I} \\
\bar{I} \\
\bar{y}\end{array}$ & $\begin{array}{l}F \\
F \\
R \\
R \\
R\end{array}$ & $\begin{array}{l}\bar{z} \\
\bar{z}\end{array}$ & $\begin{array}{l}\bar{z} \\
\bar{z} \\
\bar{z}\end{array}$ & $\begin{array}{l}\overline{\bar{F}} \\
R \\
R \\
R\end{array}$ & $\begin{array}{l}\text { R } \\
= \\
=\end{array}$ & $C$ \\
\hline- & $\begin{array}{l}\mathrm{F} \\
\mathrm{R} \\
\mathrm{F} \\
\mathrm{R} \\
\mathrm{R}\end{array}$ & $\begin{array}{l}\bar{z} \\
\bar{F} \\
\overline{-}\end{array}$ & $\begin{array}{l}\mathrm{R} \\
\mathrm{R} \\
\mathrm{R} \\
\mathrm{R} \\
\mathrm{R}\end{array}$ & $\begin{array}{l}\mathrm{F} \\
\mathrm{R} \\
\mathrm{C} \\
\mathrm{F} \\
\mathrm{R}\end{array}$ & $\begin{array}{l}\mathrm{R} \\
\bar{R} \\
\mathrm{R} \\
\mathrm{R}\end{array}$ & $\begin{array}{l}\mathrm{R} \\
\overline{\mathrm{R}} \\
\mathrm{R} \\
\end{array}$ & $\begin{array}{l}\bar{I} \\
\bar{z}\end{array}$ & \begin{tabular}{|l}
$\mathrm{F}$ \\
$\mathrm{R}$ \\
$\mathrm{C}$ \\
$\mathrm{R}$ \\
\end{tabular} & $\begin{array}{l}\mathrm{R} \\
\mathrm{F} \\
\mathrm{R} \\
\mathrm{F} \\
-\end{array}$ & $\begin{array}{l}\text { C } \\
\text { A } \\
\text { C } \\
\text { F } \\
\text { R }\end{array}$ & $\begin{array}{l}\text { C } \\
\text { A } \\
\text { A } \\
\text { C } \\
\text { C }\end{array}$ & $\begin{array}{l}\mathrm{F} \\
\mathrm{R} \\
\mathrm{F} \\
\mathrm{R} \\
\mathrm{R}\end{array}$ & $\begin{array}{l}\bar{z} \\
\bar{z} \\
\overline{-}\end{array}$ & - & $\begin{array}{l}\bar{z} \\
\bar{z}\end{array}$ & $F$ & $\begin{array}{l}\overline{-} \\
\bar{R} \\
\mathrm{R} \\
-\end{array}$ & $\bar{z}$ & $\begin{array}{l}\bar{z} \\
\bar{z}\end{array}$ & $\begin{array}{l}\bar{z} \\
\bar{z} \\
\bar{y}\end{array}$ & $\begin{array}{l}\bar{I} \\
\bar{I}\end{array}$ & $\begin{array}{l}F \\
R \\
F \\
R \\
R\end{array}$ & $\bar{z}$ & $\begin{array}{l}\bar{z} \\
\bar{z}\end{array}$ & $\begin{array}{l}\mathrm{R} \\
= \\
=\end{array}$ & $=$ & $\mathbf{A}$ \\
\hline- & $\begin{array}{l}\mathrm{F} \\
\mathrm{F} \\
\mathrm{C} \\
\mathrm{F} \\
\mathrm{R}\end{array}$ & $\begin{array}{l}\mathbf{R} \\
\mathrm{R} \\
\mathrm{R} \\
\overline{\mathrm{R}}\end{array}$ & $\begin{array}{l}\mathrm{R} \\
\mathrm{R} \\
\underline{\mathbf{R}} \\
\overline{\mathrm{R}}\end{array}$ & $\begin{array}{l}\mathrm{C} \\
\mathrm{R} \\
\mathrm{F} \\
\mathrm{R} \\
\mathrm{R}\end{array}$ & $\begin{array}{l}\overline{\mathrm{R}} \\
\overline{\mathrm{R}} \\
-\end{array}$ & $\begin{array}{l}\bar{R} \\
\bar{R}\end{array}$ & $\begin{array}{l}\bar{R} \\
R \\
- \\
-\end{array}$ & $\begin{array}{l}\mathrm{F} \\
\mathrm{R} \\
\\
- \\
-\end{array}$ & $\begin{array}{l}\bar{z} \\
\bar{R}\end{array}$ & $\begin{array}{l}\bar{R} \\
R \\
R \\
-\end{array}$ & $\begin{array}{l}\mathrm{F} \\
\mathrm{F} \\
\mathrm{F} \\
\mathrm{R} \\
\mathrm{F}\end{array}$ & $\begin{array}{l}\mathrm{R} \\
- \\
\overline{-} \\
\mathrm{R}\end{array}$ & $\overline{\bar{R}}$ & $\overline{-}$ & $\frac{\bar{R}}{\bar{R}}$ & $\begin{array}{l}\bar{z} \\
\bar{z} \\
\bar{y}\end{array}$ & $\begin{array}{l}\mathrm{R} \\
\overline{-} \\
= \\
-\end{array}$ & - & $\begin{array}{l}\bar{z} \\
\bar{z}\end{array}$ & $\begin{array}{l}\bar{z} \\
\bar{z} \\
\bar{y}\end{array}$ & $\begin{array}{l}\bar{R} \\
\bar{F} \\
\bar{F}\end{array}$ & 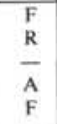 & $\begin{array}{l}\bar{z} \\
\bar{F} \\
\bar{F}\end{array}$ & $\begin{array}{l}\bar{z} \\
\bar{I}\end{array}$ & $\begin{array}{l}\mathrm{R} \\
\mathrm{R} \\
- \\
= \\
-\end{array}$ & $\begin{array}{l}\bar{R} \\
= \\
=\end{array}$ & $F$ \\
\hline- & $\begin{array}{l}\mathrm{C} \\
\mathrm{R} \\
\mathrm{F} \\
\mathrm{C} \\
\mathrm{R}\end{array}$ & $\begin{array}{l}\mathrm{R} \\
\mathrm{R} \\
\mathrm{R} \\
\mathrm{R}\end{array}$ & $\begin{array}{l}\mathrm{C} \\
\mathrm{R} \\
\mathrm{F} \\
\mathrm{F} \\
\mathrm{R}\end{array}$ & $\begin{array}{l}\mathrm{F} \\
\bar{R} \\
\mathrm{C} \\
\mathrm{F}\end{array}$ & $\begin{array}{l}R \\
R \\
R \\
R\end{array}$ & $\begin{array}{l}\bar{I} \\
\bar{R} \\
\bar{z}\end{array}$ & $\begin{array}{l}\mathrm{R} \\
\bar{z} \\
\mathbf{z}\end{array}$ & $\begin{array}{l}\bar{z} \\
\bar{z} \\
\bar{R}\end{array}$ & $\bar{I}$ & $\begin{array}{l}\bar{z} \\
\bar{I}\end{array}$ & $\begin{array}{l}R \\
F \\
\bar{C} \\
F\end{array}$ & $\begin{array}{l}\mathrm{C} \\
\mathrm{F}\end{array}$ & $\begin{array}{l}\bar{z} \\
\bar{z} \\
\bar{z}\end{array}$ & $\bar{z}$ & $\begin{array}{l}\bar{Z} \\
\bar{R} \\
\mathrm{R} \\
\overline{-}\end{array}$ & $\begin{array}{l}\bar{z} \\
\bar{z} \\
\bar{z}\end{array}$ & $\begin{array}{l}\bar{z} \\
\bar{z} \\
\bar{z}\end{array}$ & $\bar{z}$ & $\begin{array}{l}\bar{z} \\
\bar{z}\end{array}$ & $\begin{array}{l}\bar{z} \\
\bar{z} \\
\bar{y}\end{array}$ & $\begin{array}{l}F \\
R\end{array}$ & $\begin{array}{l}\bar{R} \\
\overline{-} \\
-\end{array}$ & $\begin{array}{l}R \\
R \\
\bar{F} \\
\end{array}$ & $\bar{z}$ & $\begin{array}{l}\mathrm{R} \\
\overline{\mathrm{R}} \\
-\end{array}$ & $\bar{z}$ & $\mathrm{~F}$ \\
\hline- & $\begin{array}{l}F \\
R \\
F \\
F \\
F\end{array}$ & $\begin{array}{l}\bar{R} \\
\bar{R} \\
R\end{array}$ & 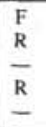 & $\begin{array}{l}\mathrm{F} \\
\mathrm{F} \\
\mathrm{F} \\
\mathrm{F} \\
-\end{array}$ & $\begin{array}{l}\bar{R} \\
R \\
F \\
R\end{array}$ & $\frac{\bar{R}}{\bar{R}}$ & $\begin{array}{l}\bar{R} \\
\mathrm{~F} \\
\mathrm{R} \\
\mathrm{F}\end{array}$ & $\frac{\bar{R}}{\bar{R}}$ & $\bar{z}$ & $\begin{array}{l}\bar{I} \\
\bar{I}\end{array}$ & $\frac{\mathrm{F}}{\mathrm{A}}$ & $\begin{array}{l}\mathrm{F} \\
\mathrm{F} \\
\mathrm{A} \\
\mathrm{C} \\
\mathrm{C}\end{array}$ & $\begin{array}{l}\mathrm{F} \\
\mathrm{F} \\
\mathrm{F} \\
\mathrm{F} \\
\mathrm{R}\end{array}$ & $\bar{z}$ & $\begin{array}{l}\overline{-} \\
\bar{R} \\
\mathrm{R} \\
\mathrm{R} \\
\mathrm{R}\end{array}$ & $\begin{array}{l}\bar{z} \\
\bar{z}\end{array}$ & $\begin{array}{l}\bar{z} \\
\bar{z}\end{array}$ & $\bar{z}$ & $\begin{array}{l}z \\
\bar{z} \\
z\end{array}$ & $\begin{array}{l}\bar{R} \\
\mathrm{R} \\
\mathrm{R} \\
-\end{array}$ & $\mathbf{R}$ & $\begin{array}{l}\overline{ } \\
\bar{z} \\
\overline{-}\end{array}$ & $\bar{z}$ & $\begin{array}{l}\frac{5}{ \pm} \\
-\end{array}$ & $\stackrel{R}{-}$ & $\overline{-}$ & $\mathrm{F}$ \\
\hline- & $\begin{array}{l}\mathrm{F} \\
\mathrm{F} \\
\mathrm{R} \\
\mathrm{R} \\
\mathrm{F}\end{array}$ & $\begin{array}{l}\bar{Z} \\
\bar{Z} \\
\mathrm{R} \\
\mathrm{R}\end{array}$ & $\begin{array}{l}\bar{R} \\
R \\
R \\
-\end{array}$ & $\begin{array}{l}\bar{R} \\
\bar{R} \\
R\end{array}$ & $\begin{array}{l}\mathrm{F} \\
\mathrm{R} \\
\mathrm{R} \\
\mathrm{R} \\
\mathrm{R}\end{array}$ & $\begin{array}{l}\mathbf{R} \\
\mathrm{R} \\
= \\
=\end{array}$ & $\begin{array}{l}\bar{z} \\
\bar{z}\end{array}$ & $\bar{z}$ & $\bar{z}$ & $\begin{array}{l}\bar{I} \\
\bar{I}\end{array}$ & $\begin{array}{l}\mathrm{F} \\
\mathrm{C} \\
\mathrm{C}\end{array}$ & $\begin{array}{l}\mathrm{F} \\
\mathrm{C} \\
\mathrm{F} \\
\mathrm{F} \\
\mathrm{C}\end{array}$ & $\begin{array}{l}\mathrm{F} \\
\mathrm{F} \\
\mathrm{R} \\
\mathrm{F} \\
\mathrm{R}\end{array}$ & $\overline{-}$ & $\begin{array}{l}\mathrm{R} \\
\mathrm{R} \\
\mathrm{R}\end{array}$ & $\begin{array}{l}\bar{z} \\
\bar{z} \\
\overline{-}\end{array}$ & $\begin{array}{l}\bar{z} \\
\bar{z}\end{array}$ & $\bar{z}$ & $\bar{I}$ & $\begin{array}{l}\bar{R} \\
\bar{R} \\
R \\
R\end{array}$ & $\underline{-}$ & $\bar{z}$ & $\bar{z}$ & $\begin{array}{l}\bar{r} \\
\bar{z}\end{array}$ & $\bar{z}$ & $\bar{z}$ & $\mathrm{~F}$ \\
\hline- & $\begin{array}{l}\mathrm{F} \\
\mathrm{R} \\
\mathrm{F} \\
\mathrm{R} \\
\mathrm{F}\end{array}$ & $\begin{array}{l}\bar{R} \\
\bar{R} \\
\bar{R}\end{array}$ & $\begin{array}{l}\mathrm{R} \\
\mathrm{R} \\
\mathrm{R} \\
\mathrm{R} \\
-\end{array}$ & $\begin{array}{l}R \\
R \\
R \\
R \\
R \\
F\end{array}$ & $\begin{array}{l}\bar{z} \\
\bar{z}\end{array}$ & $\overline{\bar{R}} \frac{\bar{R}}{\bar{R}}$ & $\begin{array}{l}\bar{z} \\
\bar{z}\end{array}$ & $\begin{array}{l}\overline{-} \\
\bar{R} \\
\bar{R}\end{array}$ & $\begin{array}{l}\bar{I} \\
\bar{z} \\
\bar{R}\end{array}$ & $\begin{array}{l}\bar{z} \\
\bar{I}\end{array}$ & $\begin{array}{l}\mathrm{C} \\
\mathrm{F} \\
\mathrm{F} \\
\mathrm{F} \\
\mathrm{R}\end{array}$ & $\begin{array}{l}\mathrm{F} \\
\mathrm{F} \\
\mathrm{F} \\
\mathrm{C} \\
\mathrm{R}\end{array}$ & $\mathrm{F}$ & $\bar{z}$ & $\begin{array}{l}\bar{I} \\
\bar{R}\end{array}$ & $\begin{array}{l}\bar{z} \\
\bar{z} \\
\bar{y}\end{array}$ & $\begin{array}{l}\bar{z} \\
\bar{z}\end{array}$ & $\begin{array}{l}\bar{z} \\
\bar{z}\end{array}$ & $\bar{z}$ & $\begin{array}{l}\overline{\bar{R}} \\
\overline{\mathrm{C}}\end{array}$ & $\overline{\bar{R}} \overline{\bar{F}}$ & $\begin{array}{l}\bar{z} \\
\bar{z} \\
\bar{z}\end{array}$ & $\begin{array}{l}\bar{z} \\
\bar{z} \\
\overline{-}\end{array}$ & $\begin{array}{l}\mathrm{F} \\
\mathrm{F} \\
-\end{array}$ & $\begin{array}{l}\bar{R} \\
R \\
R \\
R\end{array}$ & $=$ & $\begin{array}{l}F \\
F \\
F \\
F \\
F\end{array}$ \\
\hline$=$ & $\begin{array}{l}\overline{\mathrm{F}} \\
\overline{\mathrm{R}}\end{array}$ & $\begin{array}{l}- \\
\bar{R} \\
F \\
R\end{array}$ & $\begin{array}{l}\mathrm{R} \\
\overline{\bar{R}} \\
\mathrm{R} \\
\mathrm{R}\end{array}$ & $\begin{array}{l}\bar{F} \\
R \\
R \\
R\end{array}$ & $\begin{array}{l}\bar{z} \\
\bar{z} \\
\bar{z}\end{array}$ & $\begin{array}{l}\bar{I} \\
\bar{I}\end{array}$ & $\begin{array}{l}-\overline{1} \\
\bar{R} \\
R \\
R\end{array}$ & $\bar{I}$ & $\bar{z}$ & $\begin{array}{l}\bar{I} \\
\bar{I}\end{array}$ & $\begin{array}{l}\bar{R} \\
F \\
F \\
F\end{array}$ & $\begin{array}{l}\overline{-} \\
\frac{\bar{R}}{\bar{R}}\end{array}$ & $=$ & $\begin{array}{l}\overline{-} \\
\overline{\bar{F}} \\
\mathrm{~F}\end{array}$ & $\begin{array}{l}\overline{\bar{l}} \\
\overline{\mathrm{R}} \\
\mathrm{R} \\
\mathrm{R}\end{array}$ & $\begin{array}{l}\bar{z} \\
\bar{z}\end{array}$ & $\begin{array}{l}\bar{z} \\
\bar{z}\end{array}$ & $\begin{array}{l}\bar{z} \\
\bar{z}\end{array}$ & $\bar{z}$ & $\begin{array}{l}\overline{ } \\
\bar{z} \\
\overline{ }\end{array}$ & $\begin{array}{l}\bar{Z} \\
\bar{F} \\
\mathrm{R}\end{array}$ & $\begin{array}{l}\bar{z} \\
\bar{z} \\
\bar{y}\end{array}$ & $\begin{array}{l}\bar{z} \\
\bar{z} \\
\overline{-}\end{array}$ & $\begin{array}{l}\bar{z} \\
\bar{z}\end{array}$ & $\begin{array}{l}\bar{I} \\
\bar{F} \\
\mathrm{R}\end{array}$ & $\begin{array}{l}\overline{-} \\
\bar{F} \\
\mathrm{C} \\
\mathrm{F}\end{array}$ & R \\
\hline- & $\begin{array}{l}\mathrm{R} \\
\mathrm{R} \\
\mathrm{R} \\
\mathrm{R} \\
\mathrm{F}\end{array}$ & $\begin{array}{l}F \\
R \\
F \\
R \\
F\end{array}$ & $R$ & $\begin{array}{l}\mathrm{R} \\
\mathrm{R} \\
\mathrm{R} \\
\mathrm{R} \\
\mathrm{R}\end{array}$ & $\begin{array}{l}\bar{z} \\
\bar{z}\end{array}$ & $\begin{array}{l}\bar{R} \\
R \\
R \\
R\end{array}$ & $\begin{array}{l}\mathrm{R} \\
\mathrm{F} \\
\mathrm{R} \\
\mathrm{R} \\
-\end{array}$ & $\begin{array}{l}\bar{R} \\
\overline{-} \\
\overline{-}\end{array}$ & $\begin{array}{l}\bar{z} \\
\bar{z}\end{array}$ & $\begin{array}{l}\bar{z} \\
\bar{z}\end{array}$ & $\begin{array}{l}\mathrm{C} \\
\mathrm{C} \\
\mathrm{F}\end{array}$ & $\begin{array}{l}\mathrm{C} \\
\mathrm{F} \\
\mathrm{R} \\
\mathrm{A} \\
\mathrm{R}\end{array}$ & $\bar{z}$ & $\stackrel{F}{-}$ & $\begin{array}{l}\mathrm{R} \\
\mathrm{R} \\
\overline{-} \\
\overline{\mathrm{R}}\end{array}$ & $\begin{array}{l}\bar{z} \\
\bar{z} \\
\overline{-}\end{array}$ & $\begin{array}{l}\bar{z} \\
\bar{z} \\
\bar{z}\end{array}$ & - & - & $\bar{z}$ & $\begin{array}{l}\mathrm{F} \\
\mathrm{C} \\
\bar{C} \\
\mathrm{~F}\end{array}$ & -1 & $=$ & $\bar{z}$ & $\begin{array}{l}F \\
R\end{array}$ & C & $\begin{array}{l}R \\
R \\
R \\
F\end{array}$ \\
\hline 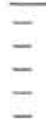 & $\begin{array}{l}\text { R } \\
\bar{z} \\
\bar{F}\end{array}$ & $\begin{array}{l}F \\
F \\
F \\
R \\
R\end{array}$ & ${ }_{R}^{R}$ & $\begin{array}{l}\mathrm{R} \\
\mathrm{F} \\
\mathrm{R} \\
\mathrm{A} \\
\mathrm{R}\end{array}$ & $\begin{array}{l}\bar{z} \\
\bar{z}\end{array}$ & $\begin{array}{l}R \\
R \\
= \\
=\end{array}$ & $\bar{z}$ & $\bar{z}$ & $\bar{z}$ & $\begin{array}{l}\bar{I} \\
\bar{z} \\
\bar{y}\end{array}$ & $\begin{array}{l}R \\
F \\
F \\
R \\
R\end{array}$ & $\begin{array}{l}\mathbf{R} \\
\mathrm{R} \\
-\end{array}$ & $\begin{array}{l}\bar{z} \\
\bar{z}\end{array}$ & $\begin{array}{l}\bar{F} \\
C \\
C \\
F\end{array}$ & $\begin{array}{l}\mathbf{R} \\
\mathrm{F} \\
\overline{\mathrm{F}}\end{array}$ & $\begin{array}{l}\bar{z} \\
\bar{z}\end{array}$ & $\begin{array}{l}\bar{z} \\
\bar{z}\end{array}$ & $\begin{array}{l}\bar{z} \\
\bar{z}\end{array}$ & $\bar{z}$ & $\begin{array}{l}\bar{z} \\
\bar{z}\end{array}$ & $\begin{array}{l}\mathrm{F} \\
\mathrm{R} \\
\mathrm{R} \\
-\end{array}$ & $\begin{array}{l}\bar{z} \\
\bar{z}\end{array}$ & $\begin{array}{l}\bar{z} \\
\bar{z}\end{array}$ & $\begin{array}{l}\bar{z} \\
\bar{z}\end{array}$ & $\begin{array}{l}\mathrm{R} \\
\mathrm{F} \\
\mathrm{R}\end{array}$ & C & $c$ \\
\hline
\end{tabular}


Table 6. Occurrence of selected diatom species observed from Hole 737B.

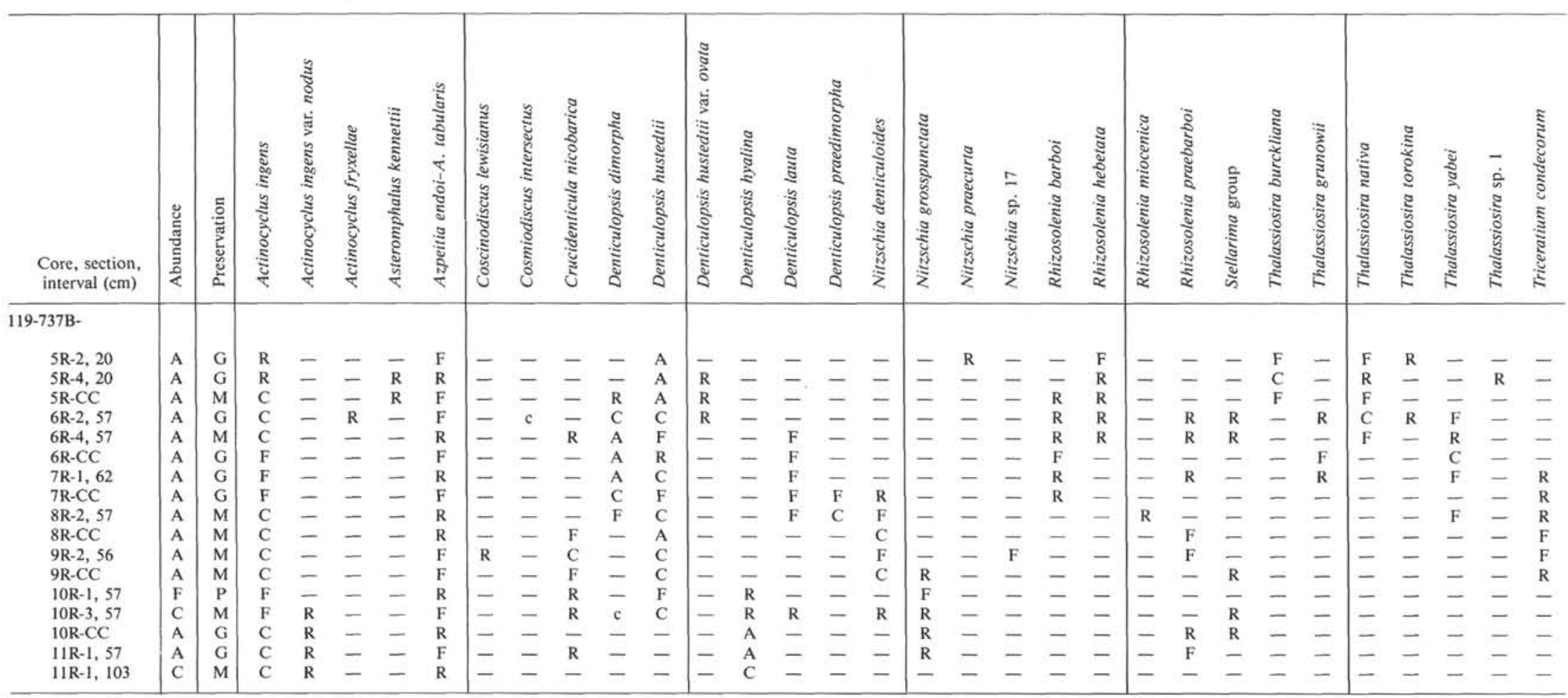

Note: Abundance: $\mathrm{A}=$ abundant, $\mathrm{C}=$ common, $\mathrm{F}=$ few, $\mathrm{R}=$ rare; preservation: $\mathrm{G}=$ good, $\mathrm{M}=$ moderate, $\mathrm{P}=$ poor; lowercase letters indicate reworking. 
recovery was essentially $100 \%$ in Cores $119-744 \mathrm{~B}-4 \mathrm{H}$ through 119-744B-9H (21.5 to 78.5 mbsf; Fig. 4) and considerably less in the equivalent section cored in Hole 744A (Barron, Larsen, et al., 1989), this interval is shown from Hole 744B in Figure 4 rather than from Hole 744A.

The upper three cores from Holes 744A and 744B contain a condensed lower Pliocene through Quaternary sequence. Although the upper $8 \mathrm{~cm}$ of Hole 744B is assigned to NSOD Zone 21 , the core-catcher samples from the first core of both holes are placed in NSOD Zone 20 . The remaining portions of the upper three cores are assigned to the following intervals: NSOD Zone 19 (119-744A-2H-2, $150 \mathrm{~cm})$, NSOD Zone 17 (119-744A-2HCC), NSOD Zone 16 (119-744B-2H-3, $50 \mathrm{~cm}$, to $119-744 \mathrm{~B}-3 \mathrm{H}-2$, $33 \mathrm{~cm})$, and NSOD Zone 15 (119-744B-3H-2, $33 \mathrm{~cm}$, to 119 744B-3H-CC).

A hiatus spanning the interval from at least 4.2 to $5.6 \mathrm{Ma}$ occurs between Samples 119-744B-3H-CC and 119-744B-4H-1, $70 \mathrm{~cm}$, based on the identification of the lower normal event of Chron C3A in the lower sample and the occurrence of Nitzschia barronii in the upper sample. The presence of Thalassiosira praeconvexa in Sample 119-744B-4H-2, $24 \mathrm{~cm}$, the occurrence of Thalassosira miocenica and Thalassiosira convexa in the interval from Samples 119-744B-4H-1, $70 \mathrm{~cm}$, through 119-744B-4H-2, $37 \mathrm{~cm}$, (Table 8) and the identification of normal magnetic polarity throughout the upper part of Core 119-744B-4H (Barron, Larsen, et al., 1989) argue for recognition of the lower normal event of Chron C3A (Fig. 4). This hiatus corresponds to the boundary between lithologic Unit I, a diatom ooze, and Unit II, a nannofossil ooze according to Barron, Larsen, et al. (1989).

A major hiatus is present between Samples 119-744B-4H-2, $37 \mathrm{~cm}$, and $119-744 \mathrm{~B}-4 \mathrm{H}-2,90 \mathrm{~cm}$, spanning the interval from about 5.9 to $9.3 \mathrm{Ma}$ (Fig. 4). This is suggested by the first occurrences of Thalassiosira miocenica and Thalassiosira convexa in Sample 119-744B-4H-2, $37 \mathrm{~cm}$, the last occurrences of Asteromphalus kennettii and Denticulopsis hustedtii var. ovata in Sample 119-744B-4H-2, $90 \mathrm{~cm}$, and interpolation upsection from the first occurrence of A. kennettii (9.6 Ma) in Sample 119-744B$4 \mathrm{H}-6,60-62 \mathrm{~cm}$. This hiatus removes most of NSOD Zone 12 and the upper part of NSOD Zone 11. No lithologic changes were observed associated with this hiatus (Barron, Larsen, et al., 1989).

The remaining portion of Core 119-744B-4H and the upper portion of Core 119-744B-5H (down to Sample 119-744B-5H-3, $60 \mathrm{~cm}$ ) are assigned to NSOD Zone 11. The last common occurrence of Denticulopsis dimorpha in Sample 119-744B-5H-2, 60 $\mathrm{cm}$, approximates the base of this zone and the top of the underlying NSOD Zone 10 . This event also correlates with C5N-1 (Fig. 4 ) and has an estimated age of $9.9 \mathrm{Ma}$ at Site 744.

The lower portion of Core 119-744B-7H through Core 119. $744 \mathrm{~B}-8 \mathrm{H}$ consists of a stratigraphic interval containing NSOD Zone 9 through NSOD Zone 7 and the upper portion of NSOD Zone 6. NSOD Zone 9 extends from immediately below the first occurrence of $D$. dimorpha in Sample 119-744B-7H-2, $60 \mathrm{~cm}$, to the first occurrence of Denticulopsis praedimorpha in Sample 119-744B-7H-3, $70 \mathrm{~cm}$. Both of these species have an abrupt first occurrence and are calibrated to the paleomagnetic results (Keating, this volume). The first occurrence of $D$. dimorpha correlates with Subchron C5AN-2 and has an estimated age of 11.9-12.0 Ma. The first occurrence of $D$. praedimorpha correlates with C5AN-2 to C5AN-3 and has an estimated age of 12.0-12.5 Ma.

NSOD Zone 8 extends downcore from immediately below the first occurrence of D. praedimorpha in Sample 119-744B-7H-3, $70 \mathrm{~cm}$, to the first occurrence of Nitzschia denticuloides in Sample $119-744 \mathrm{~B}-7 \mathrm{H}-3,70 \mathrm{~cm}$. However, it should be noted that $N$. denticuloides has a sporadic occurrence in Hole 744B and the placement of this boundary is tentative. Although Gersonde and
Burckle (1990) and Weaver and Gombos (1981) indicated that the last occurrence of Nitzschia grossepunctata corresponds with the first occurrence of $\mathrm{N}$. denticuloides, these two events are diachronous at Site 744. The last occurrence of $N$. grossepunctata occurs in Sample 119-744B-7H-3, $70 \mathrm{~cm}$, stratigraphically above the first $N$. denticuloides.

The interval from the first occurrence of $N$. denticuloides to the first occurrence of Denticulopsis hustedtii represents NSOD Zone 7. The first common occurrence of this latter species is placed in Sample 119-744B-7H-5, $70 \mathrm{~cm}$, and has an estimated age of 14.0 Ma (Gersonde and Burckle, 1990). This interval is characterized by abundant specimens of Actinocyclus ingens and D. hustedtii and common specimens of Azpeitia tabularis, Denticulopsis hyalina, and Nitzschia grossepunctata.

The first occurrence of Actinocyclus ingens in Sample 119. $744 \mathrm{~B}-8 \mathrm{H}-4,60 \mathrm{~cm}$, marks the base of NSOD Zone 6 , which represents the interval from Samples 119-744B-7H-6, $70 \mathrm{~cm}$, through $119-744 \mathrm{~B}-8 \mathrm{H}-4,60 \mathrm{~cm}$. NSOD Zone 6 is generally characterized by few to common specimens of $A$. ingens and Denticulopsis maccollumii, few specimens of $N$. grossepunctata and Synedra jouseana, and rare to few specimens of Rhizosolenia praebarboi.

NSOD Zone 5 extends from Samples 119-744B-8H-5, $60 \mathrm{~cm}$, through 119-744B-8H-CC. The first occurrence of Denticulopsis maccollumii marks the base of this zone. At Site 744 the abrupt and abundant first occurrence of this species is readily recognized. The estimated age of this event is $17.2 \mathrm{Ma}$.

The base of NSOD Zone 4, which is defined at the first occurrence of Crucidenticula nicobarica in Sample 119-744B $9 \mathrm{H}-4,58 \mathrm{~cm}$, has an estimated age of $17.8 \mathrm{Ma}$ based on correlation of this event to the paleomagnetostratigraphy at this site.

NSOD Zone 3 is assigned to the remainder of the sequence recovered, from Core 119-744B-9H to the uppermost portion of Core 119-744A-11H. The first occurrence of Thalassiosira fraga, marking the base of this zone, occurs in Sample 119-744A-11H-1, $63-65 \mathrm{~cm}$, and is calibrated to magnetic polarity event $\mathrm{C} 6 \mathrm{~N}-1$, resulting in an estimated age of 20.3 to $20.4 \mathrm{Ma}$. The last occurrence of Thalassiosira spumellaroides is placed in the lower portion of this zone in Sample 119-744A-10H-CC.

NSOD Zone 2 is defined as the interval from the first occurrence of $T$. fraga (20.7-20.9 Ma) to the first occurrence of $T$. spumellaroides (21.0-21.2 Ma). The base of this zone is placed in Sample 119-744A-11 H-4, 63-65 cm. Both the last occurrences of Rocella gelida and Rocella schraderi occur in the lower portion of this zone. Similar to the observations of Gersonde and Burckle (1990), specimens of Synedra jouseana were observed to be few to common within this interval.

NSOD Zone 1 is assigned to the interval represented by Samples $119-744 \mathrm{~A}-11 \mathrm{H}-5 \mathrm{H}, 63-64 \mathrm{~cm}$, to $119-744 \mathrm{~A}-11 \mathrm{H}-\mathrm{CC}$. Raphidodiscus marylandicus occurs within this interval, as do Synedra jouseana and Rocella gelida.

The occurrences of both $R$. gelida and $R$. vigilans allow identification the $R$. gelida and $R$. vigilans Zones. The first occurrence of $R$. gelida in Sample 119-744A-13H-1, 60-62 cm, marks the base of the $R$. gelida Zone. The first occurrence of $R$. gelida correlates with Chron $\mathrm{C} 8$ and has an estimated age of 27.4-27.6 Ma. The first occurrence of $R$. schraderi in Sample 119-744B$12 \mathrm{H}-5$ correlates with Chron C7N; $25.5-25.7 \mathrm{Ma}$. The first occurrence of Nitzschia maleinterpretaria correlates with Chron C6AAR-2 (22.4-22.5 Ma). The last occurrence of $R$. vigilans also occurs in this zone.

The $R$. vigilans Zone extends from Samples 119-744A-13H-2, $60-62 \mathrm{~cm}$, to $119-744 \mathrm{~A}-14 \mathrm{H}-4,60-62 \mathrm{~cm}$. The first occurrence of $R$. vigilans correlates with Chron $\mathrm{C} 12$ and has an estimated age of 32.7-32.9 Ma. Bogorovia veniamini has a first occurrence in 
Table 7. Occurrence of selected diatom species observed from Hole 744A.

\begin{tabular}{|c|c|c|c|c|c|c|c|c|c|c|c|c|c|c|c|c|c|c|c|c|c|c|c|c|c|}
\hline $\begin{array}{l}\text { Core, section, } \\
\text { interval }(\mathrm{cm})\end{array}$ & 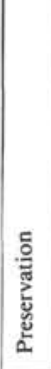 & 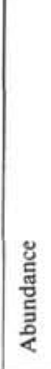 & 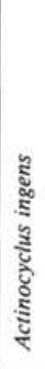 & 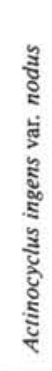 & 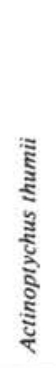 & 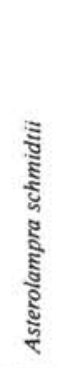 & 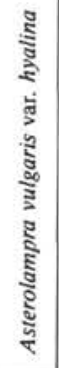 & 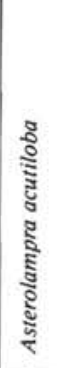 & 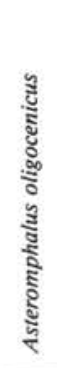 & 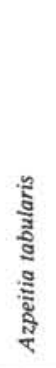 & 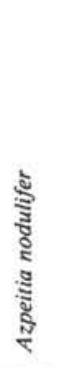 & 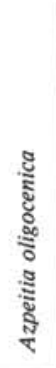 & 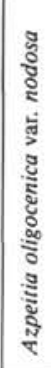 & 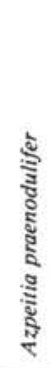 & 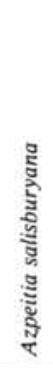 & 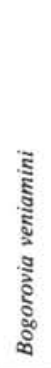 & 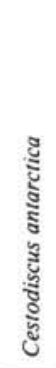 & 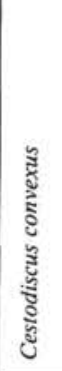 & 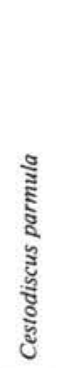 & 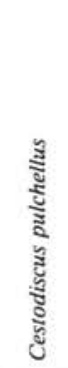 & 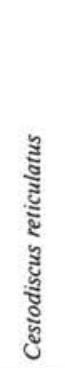 & 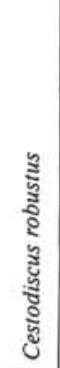 & 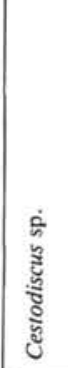 & 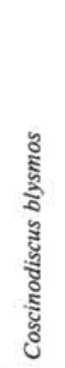 & 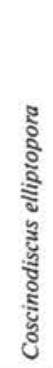 \\
\hline \multicolumn{26}{|l|}{$119-744 \mathrm{~A}-$} \\
\hline $\begin{array}{l}1 \mathrm{H}-\mathrm{CC} \\
2 \mathrm{H}-2,150 \\
2 \mathrm{H}-\mathrm{CC} \\
3 \mathrm{H}-\mathrm{CC} \\
4 \mathrm{H}-1,40\end{array}$ & $\begin{array}{l}\text { A } \\
\text { A } \\
\text { A } \\
\text { A } \\
\text { C }\end{array}$ & $\begin{array}{l}\text { G } \\
G \\
G \\
\text { M } \\
\text { G }\end{array}$ & $\begin{array}{l}\mathrm{A} \\
\mathrm{C} \\
\mathrm{F} \\
\mathrm{F} \\
\mathrm{R}\end{array}$ & $\begin{array}{l}= \\
\bar{z}\end{array}$ & $\begin{array}{l}\bar{y} \\
\bar{y}\end{array}$ & $\begin{array}{l}- \\
\overline{-} \\
-\end{array}$ & $\begin{array}{l}- \\
- \\
- \\
-\end{array}$ & $\begin{array}{l}\overline{-} \\
\overline{-} \\
-\end{array}$ & $\begin{array}{l}- \\
- \\
- \\
-\end{array}$ & $\begin{array}{l}\frac{F}{F} \\
\frac{-}{-}\end{array}$ & $\begin{array}{l}- \\
\overline{-} \\
- \\
-\end{array}$ & $\begin{array}{l}\bar{z} \\
\bar{z} \\
=\end{array}$ & $\begin{array}{l}- \\
\overline{-} \\
-\end{array}$ & $\begin{array}{l}\overline{-} \\
\bar{z} \\
\overline{-}\end{array}$ & $\begin{array}{l}\bar{z} \\
\overline{-} \\
-\end{array}$ & $\begin{array}{l}\bar{z} \\
\bar{z} \\
\overline{-}\end{array}$ & $\begin{array}{l}\bar{z} \\
\bar{z} \\
\overline{-}\end{array}$ & $\begin{array}{l}= \\
= \\
=\end{array}$ & $\begin{array}{l}\bar{z} \\
\overline{-} \\
\overline{-}\end{array}$ & $\begin{array}{l}\overline{-} \\
\overline{-} \\
\end{array}$ & $\begin{array}{l}z \\
z \\
z\end{array}$ & $\begin{array}{l}\bar{z} \\
\bar{z}\end{array}$ & $\begin{array}{l}\overline{ } \\
\bar{z} \\
\overline{-}\end{array}$ & $\begin{array}{l}\bar{z} \\
\bar{z}\end{array}$ & $\begin{array}{l}R \\
R \\
F \\
- \\
-\end{array}$ \\
\hline $\begin{array}{l}4 \mathrm{H}-\mathrm{CC} \\
5 \mathrm{H}-\mathrm{CC} \\
6 \mathrm{H}-\mathrm{CC} \\
7 \mathrm{H}-1,60 \\
7 \mathrm{H}-2,60\end{array}$ & $\begin{array}{l}\mathrm{A} \\
\mathrm{A} \\
\mathrm{C}\end{array}$ & $\begin{array}{l}\mathrm{M} \\
\mathrm{G} \\
\mathrm{G} \\
\mathrm{M} \\
\mathrm{M}\end{array}$ & $\begin{array}{l}-\bar{c} \\
\mathrm{C} \\
\mathrm{C} \\
\mathrm{C} \\
\mathrm{C}\end{array}$ & $\begin{array}{l}\overline{-} \\
\bar{z} \\
\mathrm{R} \\
\mathrm{F}\end{array}$ & $\begin{array}{l}\bar{z} \\
\bar{y} \\
\bar{y}\end{array}$ & $\begin{array}{l}z \\
\bar{z} \\
-\end{array}$ & $\begin{array}{l}- \\
- \\
- \\
- \\
-\end{array}$ & $\begin{array}{l}- \\
\overline{-} \\
\overline{-}\end{array}$ & $\begin{array}{l}- \\
\bar{z} \\
\overline{-}\end{array}$ & $\begin{array}{l}\mathrm{F} \\
\mathrm{F} \\
\mathrm{C} \\
\mathrm{F}\end{array}$ & $\begin{array}{l}\overline{-} \\
\overline{\mathrm{R}} \\
\overline{-}\end{array}$ & $\begin{array}{l}\bar{z} \\
\bar{z} \\
\overline{-}\end{array}$ & $\begin{array}{l}- \\
- \\
- \\
-\end{array}$ & $\begin{array}{l}\bar{z} \\
\bar{z} \\
\overline{-}\end{array}$ & $\begin{array}{l}\bar{z} \\
\bar{z} \\
\overline{-}\end{array}$ & $\begin{array}{l}z \\
z \\
z\end{array}$ & $\begin{array}{l}- \\
- \\
\overline{-} \\
-\end{array}$ & $\begin{array}{l}\bar{z} \\
\bar{z} \\
\overline{-}\end{array}$ & $\begin{array}{l}- \\
= \\
= \\
-\end{array}$ & $\begin{array}{l}\overline{-} \\
\overline{-} \\
-\end{array}$ & $\begin{array}{l}\bar{z} \\
\bar{z} \\
\bar{z}\end{array}$ & $\begin{array}{l}\bar{z} \\
\bar{z} \\
-\end{array}$ & $\begin{array}{l}\bar{z} \\
\bar{z} \\
\bar{z}\end{array}$ & $\begin{array}{l}\bar{z} \\
\bar{z}\end{array}$ & $\begin{array}{l}\bar{z} \\
\bar{z} \\
\overline{-}\end{array}$ \\
\hline $\begin{array}{l}7 \mathrm{H}-3,60 \\
7 \mathrm{H}-4,60 \\
7 \mathrm{H}-5,60 \\
7 \mathrm{H}-6,46 \\
7 \mathrm{H}-\mathrm{CC}\end{array}$ & $\begin{array}{l}\text { A } \\
\text { A } \\
\text { C }\end{array}$ & $\begin{array}{l}\mathrm{F} \\
\mathrm{F} \\
\mathrm{M} \\
\mathrm{M} \\
\mathrm{P}\end{array}$ & - & $\begin{array}{l}\overline{-} \\
\bar{F} \\
R \\
R\end{array}$ & $\begin{array}{l}\overline{ } \\
\bar{y} \\
\end{array}$ & $\begin{array}{l}\bar{z} \\
\overline{-} \\
-\end{array}$ & $\begin{array}{l}- \\
- \\
- \\
- \\
-\end{array}$ & $\begin{array}{l}F \\
F \\
- \\
- \\
-\end{array}$ & $\begin{array}{l}\overline{-} \\
\overline{-} \\
-\end{array}$ & $\begin{array}{l}\bar{z} \\
\bar{z} \\
\overline{-}\end{array}$ & $\begin{array}{l}- \\
\overline{-} \\
\overline{-}\end{array}$ & $\begin{array}{l}- \\
- \\
- \\
-\end{array}$ & $\begin{array}{l}- \\
- \\
- \\
-\end{array}$ & $\begin{array}{l}\bar{z} \\
\bar{z} \\
\overline{ }\end{array}$ & $\begin{array}{l}\overline{-} \\
\overline{-} \\
\bar{R}\end{array}$ & $\begin{array}{l}\bar{z} \\
\bar{z} \\
-\end{array}$ & $\begin{array}{l}- \\
- \\
- \\
-\end{array}$ & $\begin{array}{l}\bar{z} \\
\bar{z} \\
\bar{z}\end{array}$ & $\begin{array}{l}\bar{z} \\
\bar{z} \\
\overline{-}\end{array}$ & $\begin{array}{l}\overline{-} \\
\overline{-} \\
\overline{-}\end{array}$ & $\begin{array}{l}\bar{z} \\
\bar{z} \\
\bar{z}\end{array}$ & $\begin{array}{l}- \\
- \\
- \\
- \\
-\end{array}$ & $\begin{array}{l}\overline{-} \\
\bar{z} \\
\overline{-}\end{array}$ & $\begin{array}{l}\bar{z} \\
\bar{z} \\
\bar{z}\end{array}$ & $\begin{array}{l}\bar{z} \\
\bar{z} \\
\overline{-}\end{array}$ \\
\hline $\begin{array}{l}8 \mathrm{H}-\mathrm{CC} \\
9 \mathrm{H}-\mathrm{CC} \\
10 \mathrm{H}-1,46 \\
10 \mathrm{H}-2,46 \\
10 \mathrm{H}-3,46\end{array}$ & C & \begin{tabular}{|l|}
$\mathrm{M}$ \\
$\mathrm{M}$ \\
$\mathrm{G}$ \\
$\mathrm{G}$ \\
$\mathrm{M}$
\end{tabular} & - & $\begin{array}{l}z \\
z \\
z\end{array}$ & $\begin{array}{l}\bar{y} \\
\bar{y} \\
\end{array}$ & $\begin{array}{l}- \\
= \\
-\end{array}$ & $\begin{array}{l}- \\
- \\
- \\
-\end{array}$ & $\begin{array}{l}\overline{-} \\
\overline{-} \\
\overline{-}\end{array}$ & $\begin{array}{l}\bar{R} \\
\bar{F} \\
F\end{array}$ & $\begin{array}{l}\bar{F} \\
\overline{-} \\
\overline{-}\end{array}$ & $\begin{array}{l}- \\
\overline{-} \\
\overline{-}\end{array}$ & $\begin{array}{l}\overline{-} \\
\overline{-} \\
\bar{R}\end{array}$ & $\begin{array}{l}- \\
= \\
= \\
-\end{array}$ & $\begin{array}{l}\bar{R} \\
\overline{-} \\
-\end{array}$ & $\begin{array}{l}- \\
\bar{z} \\
\overline{-}\end{array}$ & $\begin{array}{l}\bar{z} \\
\bar{z} \\
\bar{y}\end{array}$ & $\begin{array}{l}- \\
\overline{-} \\
\overline{-}\end{array}$ & $\begin{array}{l}\bar{z} \\
\bar{z} \\
\bar{z}\end{array}$ & $\begin{array}{l}\bar{z} \\
\bar{z} \\
\bar{z}\end{array}$ & $\begin{array}{l}\bar{R} \\
\overline{-} \\
-\end{array}$ & $\begin{array}{l}\bar{z} \\
\bar{z} \\
\overline{-}\end{array}$ & $\begin{array}{l}\bar{z} \\
\bar{z} \\
\bar{z}\end{array}$ & $\begin{array}{l}\bar{z} \\
\bar{z} \\
\overline{-}\end{array}$ & $\begin{array}{l}\mathrm{R} \\
\overline{-} \\
\overline{-}\end{array}$ & $\begin{array}{l}\bar{z} \\
\bar{z} \\
-\end{array}$ \\
\hline $\begin{array}{l}10 \mathrm{H}-4,46 \\
10 \mathrm{H}-6,46 \\
10 \mathrm{H}-\mathrm{CC} \\
11 \mathrm{H}-1,63-65 \\
11 \mathrm{H}-2,63-65\end{array}$ & A & \begin{tabular}{|l}
$\mathrm{M}$ \\
$\mathrm{M}$ \\
$\mathrm{P}$ \\
$\mathrm{M}$ \\
$\mathrm{M}$ \\
\end{tabular} & $\begin{array}{l}- \\
- \\
\end{array}$ & $\begin{array}{l}z \\
\bar{z} \\
z\end{array}$ & $\begin{array}{l}\bar{y} \\
= \\
=\end{array}$ & $\begin{array}{l}- \\
z \\
- \\
-\end{array}$ & $\begin{array}{l}- \\
- \\
- \\
-\end{array}$ & $\begin{array}{l}\bar{z} \\
\bar{z} \\
\overline{-}\end{array}$ & $\begin{array}{l}\bar{R} \\
\mathrm{R}\end{array}$ & $\begin{array}{l}\bar{I} \\
\bar{z} \\
\overline{-}\end{array}$ & $\begin{array}{l}\overline{-} \\
\overline{-} \\
-\end{array}$ & $\begin{array}{l}\bar{R} \\
- \\
-\end{array}$ & $\begin{array}{l}- \\
- \\
- \\
- \\
-\end{array}$ & $\begin{array}{l}\bar{z} \\
\bar{z}\end{array}$ & $\begin{array}{l}\bar{z} \\
\bar{z} \\
\overline{-}\end{array}$ & $\begin{array}{l}\bar{z} \\
z \\
z\end{array}$ & $\begin{array}{l}- \\
- \\
- \\
-\end{array}$ & $\begin{array}{l}\bar{z} \\
\bar{z} \\
\bar{z}\end{array}$ & $\begin{array}{l}- \\
\overline{-} \\
-\end{array}$ & $\begin{array}{l}\bar{R} \\
\overline{-} \\
-\end{array}$ & $\begin{array}{l}\bar{z} \\
\bar{z} \\
-\end{array}$ & $\begin{array}{l}\bar{z} \\
\bar{z} \\
\bar{z}\end{array}$ & $\begin{array}{l}\bar{z} \\
\bar{z} \\
\bar{z}\end{array}$ & $\begin{array}{l}- \\
\overline{-} \\
- \\
-\end{array}$ & $\begin{array}{l}\bar{z} \\
\bar{z} \\
\overline{-}\end{array}$ \\
\hline $\begin{array}{l}11 \mathrm{H}-5,63-65 \\
11 \mathrm{H}-6,63-65 \\
11 \mathrm{H}-\mathrm{CC}\end{array}$ & A & \begin{tabular}{|l}
$\mathrm{G}$ \\
$\mathrm{M}$ \\
$\mathrm{M}$ \\
$\mathrm{M}$ \\
$\mathrm{M}$
\end{tabular} & $\begin{array}{l}- \\
\overline{-} \\
- \\
-\end{array}$ & $\begin{array}{l}\bar{z} \\
\bar{z} \\
\bar{z}\end{array}$ & $\begin{array}{l}\bar{y} \\
\bar{z} \\
\overline{-}\end{array}$ & $\begin{array}{l}\overline{-} \\
\bar{I} \\
\overline{-}\end{array}$ & $\begin{array}{l}- \\
- \\
- \\
-\end{array}$ & $\begin{array}{l}\bar{z} \\
\bar{z} \\
\overline{-}\end{array}$ & $\stackrel{\mathrm{R}}{-}$ & $\begin{array}{l}\bar{z} \\
\bar{z}\end{array}$ & $\begin{array}{l}\bar{z} \\
\bar{z} \\
\overline{-}\end{array}$ & $\begin{array}{l}\bar{R} \\
F \\
R \\
F\end{array}$ & $\begin{array}{l}\bar{z} \\
\bar{z} \\
\bar{z}\end{array}$ & $\begin{array}{l}\frac{\mathrm{R}}{\bar{Z}} \\
\overline{\mathrm{F}}\end{array}$ & $\begin{array}{l}\bar{z} \\
\bar{z} \\
\overline{-}\end{array}$ & $\begin{array}{l}\bar{z} \\
\bar{z} \\
\mathrm{R} \\
\mathrm{F}\end{array}$ & $\begin{array}{l}- \\
- \\
- \\
-\end{array}$ & $\begin{array}{l}\overline{-} \\
\bar{R} \\
\overline{-}\end{array}$ & $\begin{array}{l}\overline{-} \\
\overline{-} \\
-\end{array}$ & $\begin{array}{l}\bar{R} \\
R \\
R \\
R\end{array}$ & $\begin{array}{l}\bar{z} \\
\bar{z} \\
\bar{z}\end{array}$ & $\begin{array}{l}\bar{z} \\
\bar{z} \\
\bar{z}\end{array}$ & $\begin{array}{l}\bar{z} \\
\bar{z} \\
\overline{-}\end{array}$ & $\begin{array}{l}\overline{-} \\
\bar{R} \\
- \\
-\end{array}$ & $\begin{array}{l}\bar{z} \\
\bar{z} \\
\overline{-}\end{array}$ \\
\hline $\begin{array}{l}12 \mathrm{H}-5,60-62 \\
12,60-62\end{array}$ & c & \begin{tabular}{|l}
$\mathrm{M}$ \\
$\mathrm{M}$ \\
$\mathrm{M}$ \\
$\mathrm{M}$ \\
$\mathrm{M}$
\end{tabular} & $\begin{array}{l}- \\
- \\
- \\
-\end{array}$ & $\begin{array}{l}\bar{z} \\
\bar{z} \\
\bar{z}\end{array}$ & $\begin{array}{l}\overline{-} \\
\overline{-} \\
\bar{R}\end{array}$ & $\begin{array}{l}\bar{z} \\
\bar{I} \\
\overline{-}\end{array}$ & $\begin{array}{l}- \\
- \\
- \\
-\end{array}$ & $\begin{array}{l}\bar{z} \\
\bar{z} \\
\overline{-}\end{array}$ & $\overline{-}$ & $\begin{array}{l}\bar{z} \\
\bar{z} \\
\overline{-}\end{array}$ & $\begin{array}{l}\bar{z} \\
\bar{z} \\
-\end{array}$ & $\begin{array}{l}\mathrm{F} \\
\bar{R} \\
\mathrm{~F} \\
\mathrm{~F}\end{array}$ & $\begin{array}{l}\bar{z} \\
\bar{z} \\
\bar{z}\end{array}$ & $\bar{z}$ & $\begin{array}{l}\bar{z} \\
\bar{z} \\
\overline{-}\end{array}$ & $\begin{array}{l}F \\
- \\
- \\
-\end{array}$ & $\begin{array}{l}- \\
- \\
- \\
-\end{array}$ & $\bar{z}$ & $\begin{array}{l}\bar{z} \\
\bar{z} \\
\overline{-}\end{array}$ & $\begin{array}{l}\mathrm{R} \\
\overline{-} \\
\overline{\mathrm{R}}\end{array}$ & $\begin{array}{l}\bar{z} \\
\bar{z} \\
\bar{z}\end{array}$ & $\begin{array}{l}\bar{z} \\
\bar{z} \\
\bar{z}\end{array}$ & $\begin{array}{l}\bar{z} \\
\bar{z}\end{array}$ & $\begin{array}{l}\bar{z} \\
\bar{z} \\
\bar{z}\end{array}$ & $\begin{array}{l}\bar{z} \\
\bar{z}\end{array}$ \\
\hline $\begin{array}{l}12 \mathrm{H}-6,60-62 \\
12 \mathrm{H}-7,61-63 \\
12 \mathrm{H}-\mathrm{CC} \\
13 \mathrm{H}-1,60-62 \\
13 \mathrm{H}-2,60-62\end{array}$ & C & $\begin{array}{l}\mathrm{M} \\
\mathrm{M} \\
\mathrm{M}\end{array}$ & $\begin{array}{l}- \\
z \\
- \\
-\end{array}$ & $\begin{array}{l}z \\
\bar{z} \\
\bar{z}\end{array}$ & $\begin{array}{l}\bar{z} \\
\bar{z} \\
\end{array}$ & $\begin{array}{l}\bar{z} \\
\bar{z} \\
-\end{array}$ & $\begin{array}{l}- \\
- \\
- \\
- \\
-\end{array}$ & $\begin{array}{l}\bar{z} \\
\bar{z} \\
\overline{-}\end{array}$ & $=$ & $\begin{array}{l}\bar{z} \\
\bar{z} \\
\bar{z}\end{array}$ & $\begin{array}{l}- \\
\bar{z} \\
\overline{-}\end{array}$ & $\begin{array}{l}\overline{-} \\
\bar{R} \\
\bar{R}\end{array}$ & $\begin{array}{l}\overline{\mathrm{R}} \\
\overline{-} \\
-\end{array}$ & $\bar{z}$ & $\begin{array}{l}\bar{z} \\
\bar{z} \\
\overline{-}\end{array}$ & $\begin{array}{l}\overline{-} \\
\bar{R} \\
\bar{R}\end{array}$ & $\begin{array}{l}- \\
- \\
- \\
-\end{array}$ & $\bar{z}$ & $\begin{array}{l}\overline{\bar{z}} \\
\overline{\bar{F}}\end{array}$ & $\begin{array}{l}\bar{R} \\
R \\
R \\
-\end{array}$ & $\begin{array}{l}\bar{z} \\
\bar{z} \\
\bar{z}\end{array}$ & $\begin{array}{l}\bar{z} \\
\bar{z}\end{array}$ & $\begin{array}{l}\bar{z} \\
\bar{z}\end{array}$ & $\begin{array}{l}\bar{z} \\
\bar{z} \\
\bar{z}\end{array}$ & $\begin{array}{l}\bar{z} \\
\bar{z} \\
\overline{-}\end{array}$ \\
\hline $\begin{array}{l}13 \mathrm{H}-4,60-62 \\
13 \mathrm{H}-5,60-62 \\
13 \mathrm{H}-\mathrm{CC} \\
14 \mathrm{H}-1,64-66 \\
14 \mathrm{H}-2,60-62\end{array}$ & $F$ & $\begin{array}{l}\mathrm{M} \\
\mathrm{G} \\
\mathrm{M} \\
\mathrm{M} \\
\mathrm{M}\end{array}$ & - & $\begin{array}{l}\bar{z} \\
\bar{z} \\
\bar{z}\end{array}$ & $\begin{array}{l}\overline{-} \\
\bar{R} \\
\overline{-}\end{array}$ & $\begin{array}{l}- \\
\overline{-} \\
\bar{R}\end{array}$ & $\begin{array}{l}- \\
- \\
- \\
-\end{array}$ & $\bar{z}$ & $\overline{-}$ & $\begin{array}{l}\bar{z} \\
\bar{z} \\
\overline{-}\end{array}$ & $\begin{array}{l}\bar{z} \\
\bar{z} \\
\overline{-}\end{array}$ & $\begin{array}{l}\overline{-} \\
\bar{R} \\
- \\
-\end{array}$ & $\begin{array}{l}\bar{z} \\
\bar{z} \\
\bar{z}\end{array}$ & $\bar{z}$ & $\begin{array}{l}\bar{z} \\
\bar{z} \\
\bar{y}\end{array}$ & $\begin{array}{l}\bar{z} \\
\bar{z} \\
\bar{y}\end{array}$ & $\begin{array}{l}= \\
= \\
- \\
-\end{array}$ & $\begin{array}{l}\mathrm{R} \\
- \\
= \\
-\end{array}$ & $\begin{array}{l}\frac{\mathrm{R}}{\bar{R}} \\
\overline{\mathrm{R}} \\
-\end{array}$ & $\begin{array}{l}\mathrm{R} \\
\mathrm{R} \\
\mathrm{R} \\
\mathrm{R}\end{array}$ & $\begin{array}{l}\bar{z} \\
\bar{z}\end{array}$ & $\begin{array}{l}\bar{z} \\
\bar{z} \\
\bar{z}\end{array}$ & $\begin{array}{l}\bar{z} \\
\bar{z}\end{array}$ & $\begin{array}{l}\bar{z} \\
\bar{z} \\
\bar{z}\end{array}$ & $\begin{array}{l}\bar{z} \\
\bar{z} \\
\overline{-}\end{array}$ \\
\hline $\begin{array}{l}14 \mathrm{H}-4,60-62 \\
14 \mathrm{H}-5,60-62 \\
14 \mathrm{H}-\mathrm{CC} \\
15 \mathrm{H}-1,60-62 \\
15 \mathrm{H}-2,60-62\end{array}$ & A & $\begin{array}{l}\mathrm{M} \\
\mathrm{M} \\
\mathrm{M} \\
\mathrm{M} \\
\mathrm{M}\end{array}$ & - & $\begin{array}{l}\bar{z} \\
\bar{z}\end{array}$ & $\begin{array}{l}\bar{z} \\
\overline{-}\end{array}$ & $\begin{array}{l}R \\
R \\
R \\
R\end{array}$ & $\begin{array}{l}- \\
- \\
\overline{-}\end{array}$ & $\begin{array}{l}\mathrm{R} \\
\overline{-} \\
\mathrm{R} \\
\mathrm{R}\end{array}$ & $\begin{array}{l}\bar{z} \\
\overline{-} \\
\end{array}$ & $\begin{array}{l}\bar{z} \\
\overline{-}\end{array}$ & $\begin{array}{l}\overline{-} \\
\bar{z} \\
\overline{-}\end{array}$ & $\begin{array}{l}\bar{R} \\
\bar{R} \\
-\end{array}$ & $\begin{array}{l}\bar{z} \\
\bar{z} \\
\bar{z}\end{array}$ & $\begin{array}{l}\bar{z} \\
\overline{-}\end{array}$ & $\begin{array}{l}\bar{z} \\
\bar{z} \\
\end{array}$ & $\begin{array}{l}\bar{z} \\
\bar{z} \\
\overline{-}\end{array}$ & $\begin{array}{l}\bar{R} \\
- \\
- \\
-\end{array}$ & $\begin{array}{l}-\bar{R} \\
R \\
R \\
R\end{array}$ & $\begin{array}{l}\bar{z} \\
\bar{z}\end{array}$ & $\begin{array}{l}\mathrm{F} \\
\mathrm{R} \\
\mathrm{R} \\
\mathrm{R}\end{array}$ & $\begin{array}{l}\mathrm{R} \\
\mathrm{R} \\
\mathrm{R} \\
\mathrm{R} \\
\mathrm{R}\end{array}$ & $\begin{array}{l}\bar{R} \\
\overline{-} \\
-\end{array}$ & $\begin{array}{l}\bar{R} \\
\bar{R}\end{array}$ & $\begin{array}{l}\bar{z} \\
\bar{z} \\
\bar{z}\end{array}$ & $\begin{array}{l}\bar{z} \\
\bar{z} \\
\overline{-}\end{array}$ \\
\hline $\begin{array}{l}15 \mathrm{H}-4,60-62 \\
15 \mathrm{H}-5,60-62 \\
15 \mathrm{H}-7,60-62 \\
15 \mathrm{H}-\mathrm{CC} \\
16 \mathrm{H}-1,60-62\end{array}$ & $\begin{array}{l}\text { A } \\
\text { A } \\
\text { A } \\
\text { C } \\
\text { A }\end{array}$ & $\begin{array}{l}\text { G } \\
\text { M } \\
\text { G } \\
\text { M } \\
\text { M }\end{array}$ & - & $\begin{array}{l}\bar{z} \\
\bar{z} \\
\overline{-}\end{array}$ & $\begin{array}{l}\bar{R} \\
\mathrm{R} \\
- \\
-\end{array}$ & $\begin{array}{l}\mathrm{R} \\
\mathrm{R} \\
\mathrm{R} \\
\mathrm{R} \\
\mathrm{R}\end{array}$ & $\begin{array}{l}\mathrm{R} \\
- \\
- \\
-\end{array}$ & $\begin{array}{l}\mathbf{R} \\
- \\
= \\
-\end{array}$ & $\begin{array}{l}\bar{z} \\
\bar{z} \\
-\end{array}$ & $\begin{array}{l}\bar{z} \\
\overline{-}\end{array}$ & $\begin{array}{l}\bar{z} \\
\bar{z} \\
\overline{-}\end{array}$ & $\begin{array}{l}\mathrm{C} \\
\mathrm{R} \\
\mathrm{R} \\
\mathrm{R}\end{array}$ & $\begin{array}{l}\mathrm{F} \\
\mathrm{F} \\
\mathrm{R} \\
\mathrm{R}\end{array}$ & $\begin{array}{l}\bar{z} \\
\bar{z} \\
\bar{z}\end{array}$ & $\begin{array}{l}\bar{z} \\
\bar{z} \\
\overline{-}\end{array}$ & $\begin{array}{l}\bar{z} \\
\bar{z} \\
\overline{-}\end{array}$ & $\begin{array}{l}\overline{\mathrm{R}} \\
\overline{-} \\
-\end{array}$ & $\begin{array}{l}\mathrm{C} \\
\mathrm{R} \\
\overline{\mathrm{F}} \\
-\end{array}$ & $\begin{array}{l}\frac{R}{F} \\
- \\
\end{array}$ & $\begin{array}{l}\mathrm{F} \\
\mathrm{F} \\
\mathrm{F} \\
\mathrm{F} \\
\mathrm{R}\end{array}$ & $\begin{array}{l}\mathrm{F} \\
\mathrm{R} \\
\mathrm{F} \\
\\
-\end{array}$ & $\begin{array}{l}\bar{R} \\
A \\
F \\
-\end{array}$ & $\begin{array}{l}\mathrm{R} \\
\overline{\mathrm{F}} \\
-\end{array}$ & $\begin{array}{l}\bar{z} \\
\bar{z} \\
=\end{array}$ & $\begin{array}{l}\bar{z} \\
\bar{z} \\
\overline{-}\end{array}$ \\
\hline $\begin{array}{l}16 \mathrm{H}-2,60-62 \\
16 \mathrm{H}-4,60-62 \\
16 \mathrm{H}-5,60-62 \\
16 \mathrm{H}-7,60-62 \\
16 \mathrm{H}-\mathrm{CC}\end{array}$ & $\begin{array}{l}\mathrm{A} \\
\mathrm{A} \\
\mathrm{A} \\
\mathrm{A} \\
\mathrm{C}\end{array}$ & \begin{tabular}{|l}
$\mathrm{C}$ \\
$\mathrm{M}$ \\
$\mathrm{M}$ \\
$\mathrm{M}$ \\
$\mathrm{P}$
\end{tabular} & - & $\begin{array}{l}\bar{z} \\
\bar{z} \\
\overline{-}\end{array}$ & $\begin{array}{l}= \\
= \\
=\end{array}$ & $\begin{array}{l}\mathbf{R} \\
\mathbf{R} \\
- \\
- \\
-\end{array}$ & $\begin{array}{l}\bar{R} \\
\overline{-} \\
\bar{R}\end{array}$ & $\begin{array}{l}\mathbf{R} \\
- \\
- \\
-\end{array}$ & $\begin{array}{l}\bar{z} \\
\overline{-}\end{array}$ & $\begin{array}{l}\bar{z} \\
\bar{z}\end{array}$ & $\begin{array}{l}\bar{z} \\
\bar{z} \\
-\end{array}$ & $\begin{array}{l}\mathrm{R} \\
\mathrm{R} \\
\mathrm{R} \\
\mathrm{R} \\
\mathrm{R}\end{array}$ & $\begin{array}{l}\bar{z} \\
\bar{z} \\
\bar{z}\end{array}$ & $\begin{array}{l}\bar{z} \\
\bar{z} \\
\bar{y}\end{array}$ & $\begin{array}{l}\bar{z} \\
\bar{y} \\
\end{array}$ & $\begin{array}{l}\bar{R} \\
\overline{-} \\
-\end{array}$ & $\begin{array}{l}\mathrm{R} \\
- \\
- \\
-\end{array}$ & $\begin{array}{l}\mathrm{R} \\
= \\
=\end{array}$ & $\begin{array}{l}\mathrm{R} \\
\mathrm{R} \\
- \\
- \\
-\end{array}$ & $\begin{array}{l}\bar{F} \\
R \\
R \\
R\end{array}$ & $\begin{array}{l}\bar{z} \\
\bar{z}\end{array}$ & $\begin{array}{l}\bar{z} \\
- \\
- \\
-\end{array}$ & $\begin{array}{l}\bar{z} \\
\bar{z} \\
\bar{z}\end{array}$ & $\begin{array}{l}\bar{z} \\
\bar{z} \\
\bar{z}\end{array}$ & $\begin{array}{l}\bar{z} \\
\bar{z} \\
\overline{-}\end{array}$ \\
\hline
\end{tabular}

Note: Abundance: $\mathrm{A}=$ abundant, $\mathrm{C}=$ common, $\mathrm{F}=$ few, $\mathrm{R}=$ rare; preservation: $\mathrm{G}=$ good, $\mathrm{M}=$ moderate, $\mathrm{P}=$ poor; lowercase letters indicate reworking. 
Table 7 (Continued).

\begin{tabular}{|c|c|c|c|c|c|c|c|c|c|c|c|c|c|c|c|c|c|c|c|c|c|c|c|c|c|c|c|}
\hline 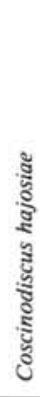 & 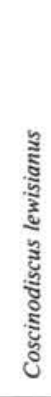 & 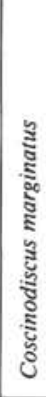 & 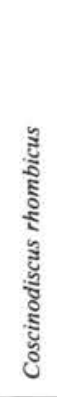 & 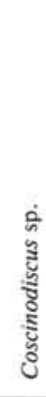 & 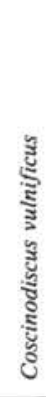 & 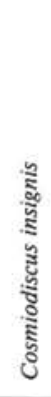 & 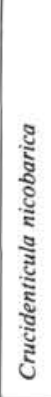 & 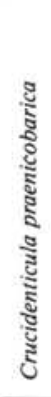 & 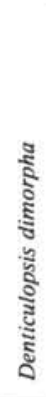 & 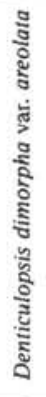 & 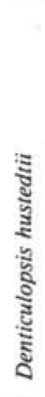 & 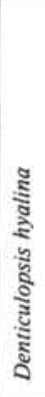 & 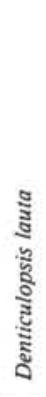 & 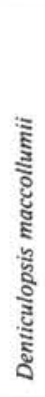 & 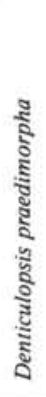 & 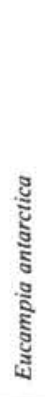 & 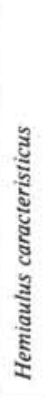 & 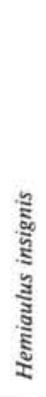 & 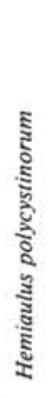 & 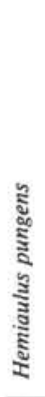 & 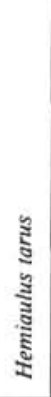 & 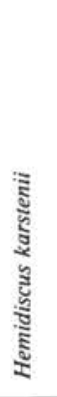 & 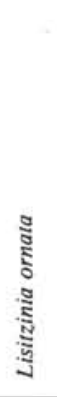 & 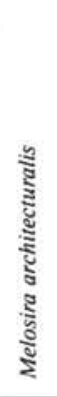 & 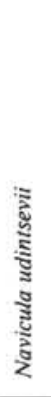 & 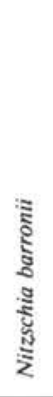 & 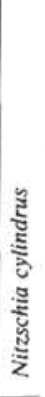 \\
\hline $\begin{array}{l}\overline{-} \\
\bar{z} \\
\overline{-}\end{array}$ & $\begin{array}{l}\bar{z} \\
\bar{z} \\
\overline{-}\end{array}$ & $\begin{array}{l}- \\
\overline{-} \\
\overline{\mathrm{F}} \\
\mathrm{F}\end{array}$ & $\begin{array}{l}- \\
\overline{-} \\
\overline{-}\end{array}$ & $\begin{array}{l}\overline{-} \\
\bar{F} \\
- \\
-\end{array}$ & $\begin{array}{l}\frac{-}{\mathrm{R}} \\
\frac{-}{-}\end{array}$ & $\begin{array}{l}\overline{-} \\
\overline{\mathrm{R}} \\
- \\
-\end{array}$ & $\begin{array}{l}- \\
z \\
z \\
-\end{array}$ & $\begin{array}{l}- \\
\bar{z} \\
\overline{-}\end{array}$ & $\begin{array}{l}- \\
\bar{R} \\
\mathrm{~F} \\
\mathrm{~F}\end{array}$ & $\begin{array}{l}\bar{z} \\
\bar{z} \\
-\end{array}$ & $\frac{\bar{F}}{\bar{F}}$ & $\begin{array}{l}\overline{-} \\
\overline{-} \\
-\end{array}$ & $\begin{array}{l}\overline{-} \\
\bar{z} \\
\overline{-}\end{array}$ & $\begin{array}{l}\bar{z} \\
\bar{z}\end{array}$ & $\overline{-}$ & $\frac{\mathrm{F}}{\overline{\mathrm{R}}}$ & $\begin{array}{l}\overline{-} \\
\overline{-} \\
-\end{array}$ & $\begin{array}{l}\bar{z} \\
\bar{z} \\
\bar{z}\end{array}$ & $\begin{array}{l}\bar{z} \\
\bar{z} \\
\overline{-}\end{array}$ & - & $\begin{array}{l}\bar{z} \\
\bar{z} \\
\overline{-}\end{array}$ & $\begin{array}{l}\overline{-} \\
\overline{\mathrm{R}} \\
\underline{-}\end{array}$ & $\begin{array}{l}\bar{z} \\
\bar{z} \\
\bar{z}\end{array}$ & $\begin{array}{l}\bar{z} \\
\bar{z} \\
\overline{-}\end{array}$ & $\begin{array}{l}\bar{z} \\
\bar{z} \\
\overline{-}\end{array}$ & $\begin{array}{l}\overline{-} \\
\overline{-} \\
\overline{-}\end{array}$ & $\frac{\mathrm{F}}{\mathrm{F}}$ \\
\hline $\begin{array}{l}\bar{z} \\
\overline{-} \\
-\end{array}$ & $\begin{array}{l}\bar{z} \\
\bar{z} \\
\overline{-}\end{array}$ & $\frac{\bar{z}}{\mathrm{R}}$ & $\begin{array}{l}\bar{z} \\
\bar{z} \\
\bar{z}\end{array}$ & $\begin{array}{l}\bar{z} \\
\bar{z} \\
\overline{-}\end{array}$ & $\overline{-}$ & $\begin{array}{l}\bar{z} \\
\bar{z} \\
\bar{z}\end{array}$ & $\begin{array}{l}\bar{R} \\
F\end{array}$ & $\begin{array}{l}\bar{z} \\
\bar{z} \\
\overline{-}\end{array}$ & $\frac{\mathrm{A}}{-}$ & $\begin{array}{l}\mathrm{R} \\
= \\
- \\
-\end{array}$ & $\mathrm{C}$ & & $\frac{-}{-}$ & $\begin{array}{l}\bar{z} \\
\overline{-}\end{array}$ & & $\begin{array}{l}\bar{z} \\
\bar{z} \\
\overline{-}\end{array}$ & - & $\underline{-}$ & $\begin{array}{l}\bar{z} \\
\bar{z} \\
\bar{z}\end{array}$ & - & $\bar{z}$ & - & - & $\begin{array}{l}\bar{y} \\
\bar{z} \\
\overline{-}\end{array}$ & $\bar{z}$ & $\frac{\mathrm{R}}{\mathrm{F}}$ & $\begin{array}{l}\bar{z} \\
\bar{z} \\
\overline{-}\end{array}$ \\
\hline $\begin{array}{l}\bar{z} \\
\overline{-}\end{array}$ & $\begin{array}{l}\overline{-} \\
\bar{R} \\
\overline{-}\end{array}$ & $\overline{\mathrm{F}}$ & $\begin{array}{l}\bar{z} \\
\bar{z} \\
\bar{z}\end{array}$ & $\begin{array}{l}\overline{-} \\
\overline{-} \\
\overline{-}\end{array}$ & $\overline{-}$ & $\begin{array}{l}\bar{z} \\
\bar{z} \\
\overline{-}\end{array}$ & - & $\bar{z}$ & $\bar{z}$ & $\begin{array}{l}\bar{z} \\
\bar{z} \\
\overline{-}\end{array}$ & $\mathrm{F}$ & $\mathrm{F}$ & $\overline{\bar{F}}$ & $\begin{array}{l}\frac{\mathrm{A}}{-} \\
\overline{\mathrm{F}}\end{array}$ & & 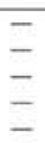 & & & $\begin{array}{l}\bar{z} \\
\bar{z}\end{array}$ & - & $\bar{z}$ & - & $\overline{-}$ & $\begin{array}{l}\bar{z} \\
\bar{z} \\
\overline{-}\end{array}$ & $\bar{z}$ & $\begin{array}{l}\overline{-} \\
\overline{-} \\
\bar{R} \\
\mathrm{R}\end{array}$ & $\begin{array}{l}\bar{z} \\
\bar{z} \\
\overline{-}\end{array}$ \\
\hline- & $\begin{array}{l}\mathbf{R} \\
\overline{-} \\
\overline{-}\end{array}$ & $\begin{array}{l}\text { F } \\
F \\
C \\
C \\
F\end{array}$ & $\begin{array}{l}\bar{z} \\
\bar{z} \\
\overline{-}\end{array}$ & $\begin{array}{l}\overline{-} \\
\overline{-} \\
-\end{array}$ & $\bar{z}$ & $\begin{array}{l}\bar{z} \\
\bar{z} \\
\overline{-}\end{array}$ & - & - & - & $=$ & - & & - & - & & $\bar{z}$ & & & $\bar{z}$ & & - & & $\overline{-}$ & $\overline{-}$ & - & $\frac{-}{-}$ & $\begin{array}{l}\bar{z} \\
\bar{z} \\
\overline{-}\end{array}$ \\
\hline$\frac{-}{-}$ & $\begin{array}{l}\bar{z} \\
\bar{z} \\
\overline{-}\end{array}$ & $F$ & $\bar{I}$ & $\begin{array}{l}\overline{-} \\
- \\
- \\
-\end{array}$ & & $\begin{array}{l}\bar{z} \\
\bar{z} \\
\overline{-}\end{array}$ & & & - & $\pi$ & - & & & $\pi$ & & - & & & - & & & & & $\begin{array}{l}\overline{-} \\
\overline{-} \\
-\end{array}$ & - & - & $\begin{array}{l}\bar{z} \\
\overline{-} \\
\overline{-}\end{array}$ \\
\hline- & - & $\mathrm{F}$ & $\bar{z}$ & $\begin{array}{l}- \\
\overline{-} \\
\overline{\mathrm{R}}\end{array}$ & & $\begin{array}{l}\overline{-} \\
\bar{z} \\
\overline{-}\end{array}$ & & & - & 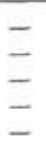 & - & & & - & & - & & & $P$ & & & & & $\begin{array}{l}\bar{z} \\
\bar{z}\end{array}$ & - & - & $\begin{array}{l}\bar{z} \\
\overline{-}\end{array}$ \\
\hline- & - & $\overline{\mathrm{R}}$ & - & $\begin{array}{l}\mathrm{R} \\
\mathrm{A} \\
\mathrm{F} \\
\mathrm{F}\end{array}$ & & $\begin{array}{l}\bar{z} \\
\bar{z}\end{array}$ & & & - & & $\overline{-}$ & & & - & & $\bar{z}$ & & & $\overline{\mathrm{R}}$ & & & & $\mathrm{F}$ & $\overline{-}$ & - & - & $\begin{array}{l}\bar{z} \\
\bar{z} \\
\overline{-}\end{array}$ \\
\hline- & $\bar{z}$ & $\mathrm{R}$ & $\bar{I}$ & $\begin{array}{l}\overline{\mathrm{F}} \\
\overline{-} \\
\overline{-}\end{array}$ & & $\begin{array}{l}\bar{z} \\
\bar{z} \\
\overline{-}\end{array}$ & & $\overline{\bar{z}}$ & - & & - & & & - & & $\overline{\bar{I}}$ & & & - & & & & - & $\bar{z}$ & - & - & $\begin{array}{l}\bar{I} \\
\bar{I}\end{array}$ \\
\hline- & $\overline{-}$ & $R$ & $\overline{\mathrm{R}}$ & $\begin{array}{l}\overline{-} \\
- \\
- \\
-\end{array}$ & & $\begin{array}{l}\overline{-} \\
\bar{z} \\
\overline{-}\end{array}$ & - & - & - & $\pi$ & - & & - & $\pi$ & & $\overline{-}$ & - & $\mathrm{F}$ & & & & - & - & $\overline{\mathrm{R}}$ & - & - & $\begin{array}{l}\bar{Z} \\
\bar{I} \\
\overline{-}\end{array}$ \\
\hline $\bar{R}$ & $\bar{z}$ & $\begin{array}{l}F \\
R \\
R \\
F \\
F\end{array}$ & $\overline{\bar{R}}$ & $\begin{array}{l}- \\
- \\
\overline{-} \\
-\end{array}$ & & - & & - & - & - & - & & - & - & & $\bar{z}$ & - & & $\bar{z}$ & & & - & - & $\overline{-}$ & - & - & $\begin{array}{l}\bar{I} \\
\overline{-} \\
\overline{-}\end{array}$ \\
\hline$\frac{\mathrm{R}}{-}$ & $\begin{array}{l}\bar{z} \\
-\end{array}$ & - & $\bar{I}$ & $\begin{array}{l}\overline{-} \\
\overline{-} \\
-\end{array}$ & $\begin{array}{l}\overline{-} \\
\overline{-}\end{array}$ & $\bar{z}$ & - & - & - & - & - & - & - & $\bar{z}$ & & $\overline{-}$ & - & & 'C & & -1 & - & - & $\frac{-}{-}$ & $\frac{\mathrm{R}}{-}$ & - & $=$ \\
\hline$\overline{\mathrm{R}}$ & $\begin{array}{l}\mathbf{R} \\
- \\
- \\
-\end{array}$ & - & $\begin{array}{l}\bar{R} \\
\text { F }\end{array}$ & $\begin{array}{l}- \\
- \\
- \\
-\end{array}$ & - & $\begin{array}{l}\bar{z} \\
\bar{z} \\
\overline{-}\end{array}$ & $\overline{-}$ & $\begin{array}{l}\bar{z} \\
\bar{z} \\
\overline{-}\end{array}$ & $\begin{array}{l}\bar{z} \\
\overline{-} \\
\overline{-}\end{array}$ & - & - & - & - & $\bar{z}$ & - & - & - & $\mathbf{F}$ & $\mathrm{F}$ & - & -1 & $\overline{-}$ & - & $\begin{array}{l}\bar{z} \\
\bar{z} \\
\bar{z}\end{array}$ & - & $\begin{array}{l}\bar{z} \\
\bar{z} \\
-\end{array}$ & $\begin{array}{l}- \\
-\end{array}$ \\
\hline
\end{tabular}


Table 7 (Continued).

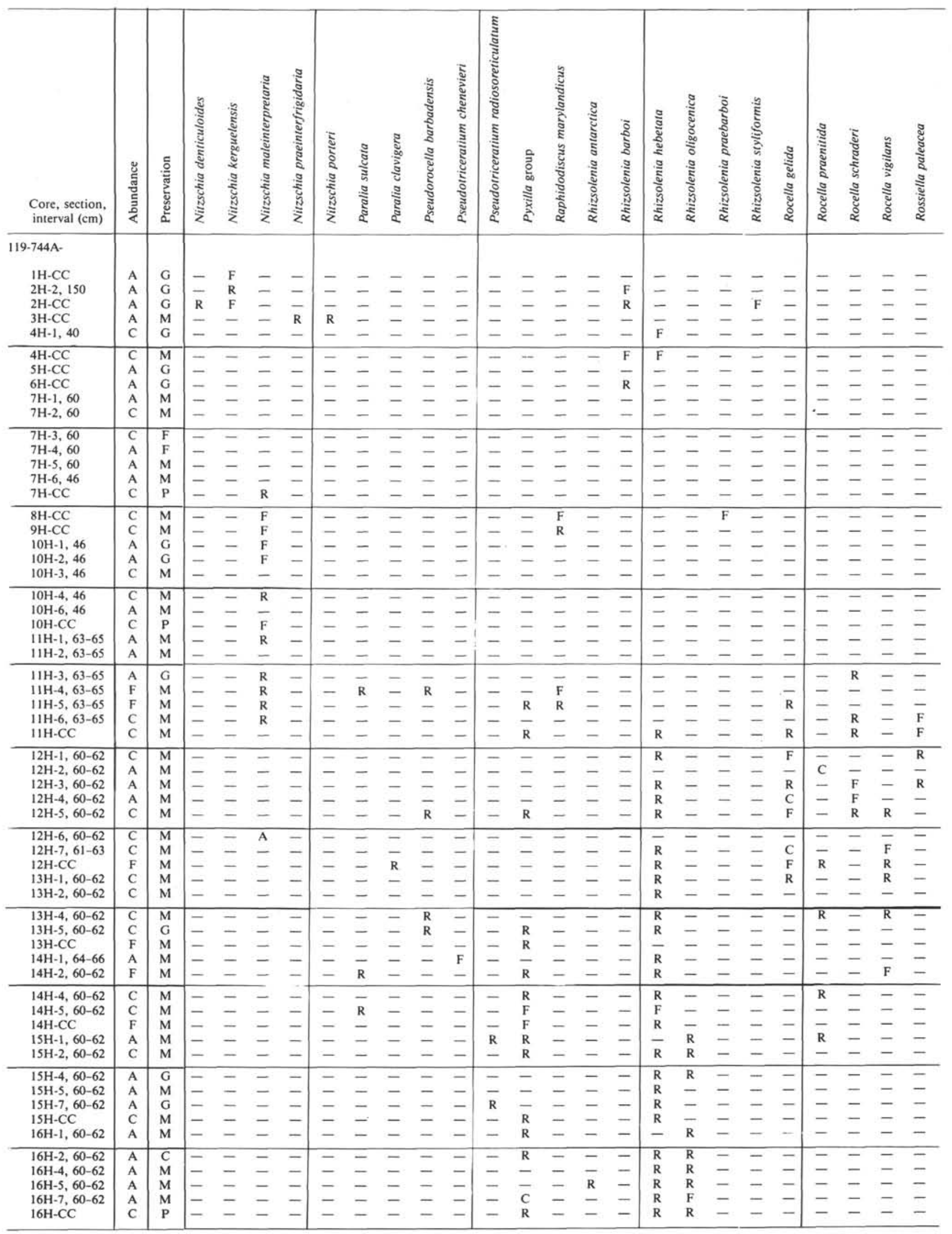


Table 7 (Continued).

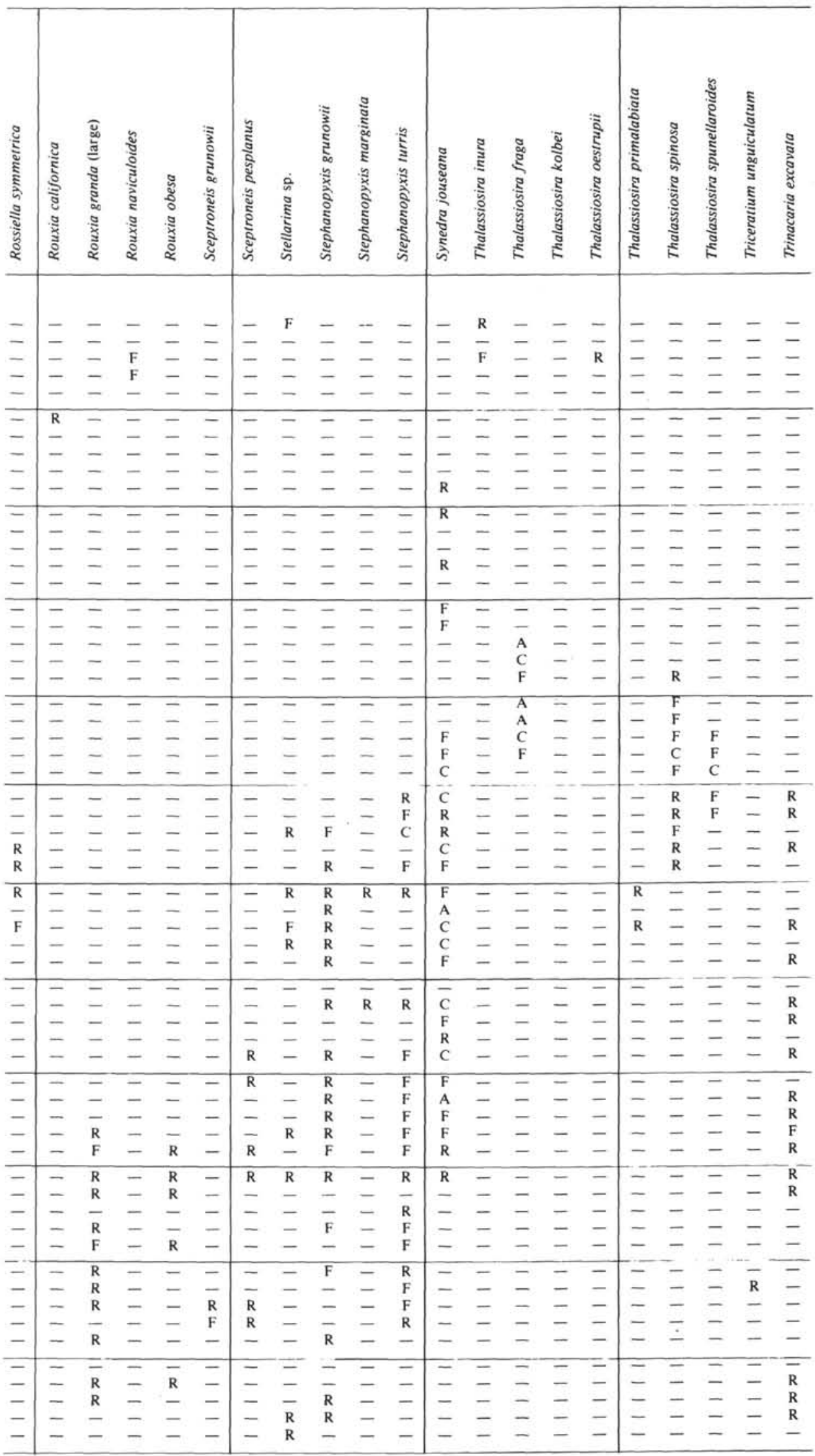


Table 8. Occurrence of selected diatom species observed from Hole 744B.

\begin{tabular}{|c|c|c|c|c|c|c|c|c|c|c|c|c|c|c|c|c|c|c|c|c|c|c|}
\hline $\begin{array}{l}\text { Core, section, } \\
\text { interval }(\mathrm{cm})\end{array}$ & 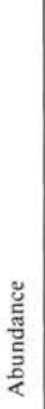 & 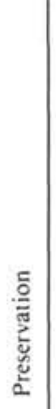 & 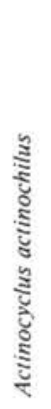 & 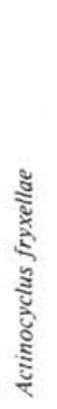 & 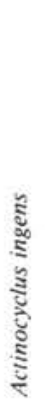 & 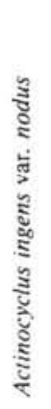 & 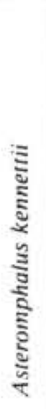 & 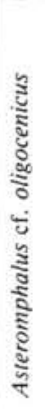 & 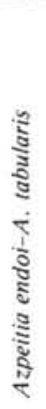 & 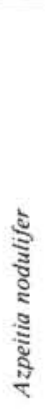 & 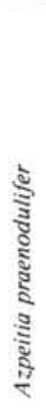 & 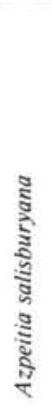 & 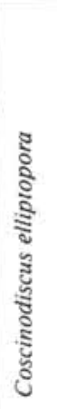 & 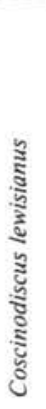 & 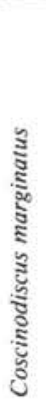 & 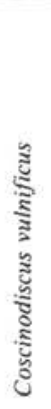 & 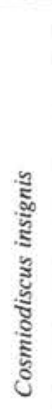 & 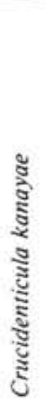 & 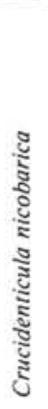 & 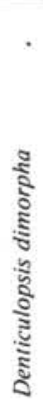 & 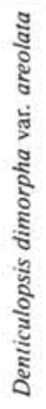 & 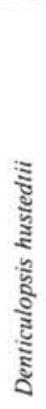 \\
\hline \multicolumn{23}{|l|}{ 119-744B- } \\
\hline $1 \mathrm{H}-1,8$ & A & G & $\mathrm{F}$ & - & - & - & - & - & $\mathrm{F}$ & - & - & - & - & - & - & - & - & - & - & - & - & - \\
\hline $1 \mathrm{H}-\mathrm{CC}$ & A & G & - & - & $\mathrm{C}$ & - & - & - & - & - & - & - & $\mathrm{F}$ & - & - & - & - & - & - & - & - & - \\
\hline $2 \mathrm{H}-3,50$ & A & G & - & - & - & - & - & - & - & - & - & - & $\mathrm{F}$ & $\mathrm{F}$ & - & $\mathrm{F}$ & $\mathrm{F}$ & - & - & - & - & $\mathrm{F}$ \\
\hline $2 \mathrm{H}-\mathrm{CC}$ & A & G & - & - & $\mathrm{R}$ & - & - & - & - & $\mathrm{F}$ & - & - & - & - & - & - & $\mathbf{R}$ & - & - & $\mathrm{F}$ & - & $\mathrm{F}$ \\
\hline $3 \mathrm{H}-2,33$ & A & G & - & - & - & - & - & - & - & - & - & - & - & - & - & - & $\mathrm{F}$ & - & - & - & - & F \\
\hline $3 \mathrm{H}-\mathrm{CC}$ & A & $G$ & - & - & - & - & - & - & - & - & - & - & - & - & - & - & - & - & - & - & - & $F$ \\
\hline $4 \mathrm{H}-1,70$ & A & G & - & - & - & - & - & - & - & - & - & - & - & - & $\mathrm{C}$ & - & - & - & - & $\mathrm{F}$ & - & $\mathrm{F}$ \\
\hline $4 \mathrm{H}-2,24$ & A & G & - & - & $\mathrm{F}$ & - & - & - & - & - & - & - & - & - & - & - & $\mathrm{R}$ & - & - & $\mathrm{F}$ & - & $\mathrm{F}$ \\
\hline $4 \mathrm{H}-2,37$ & A & G & - & - & $\mathrm{F}$ & - & - & - & - & - & - & - & - & - & $\mathrm{F}$ & - & $\mathrm{F}$ & - & - & $\mathrm{F}$ & - & $\mathrm{F}$ \\
\hline $4 \mathrm{H}-2,90$ & $\mathrm{C}$ & G & - & $\mathrm{R}$ & $\mathrm{F}$ & - & - & - & - & - & - & - & - & - & $\mathrm{F}$ & - & $\mathrm{R}$ & - & - & $\mathrm{R}$ & $\mathrm{F}$ & $\mathrm{F}$ \\
\hline $4 \mathrm{H}-3,27$ & $\mathrm{~F}$ & $\mathrm{M}$ & - & $\mathrm{R}$ & - & - & $\mathrm{R}$ & - & - & - & - & - & - & - & $\mathrm{F}$ & - & - & - & - & $\mathrm{R}$ & $\mathrm{F}$ & $\mathrm{F}$ \\
\hline $4 \mathrm{H}-3,59$ & A & G & - & $\ddot{R}$ & $\mathrm{R}$ & - & $\mathrm{F}$ & - & - & - & - & - & - & - & $\mathrm{R}$ & - & - & - & - & $\ddot{R}$ & A & $\mathrm{F}$ \\
\hline $4 \mathrm{H}-4,60$ & A & G & - & $\vec{F}$ & - & - & $\mathrm{F}$ & - & - & - & - & - & - & - & $\ddot{c}$ & - & - & - & - & $\mathrm{F}$ & - & A \\
\hline $4 \mathrm{H}-5,60$ & A & $M$ & - & $\mathrm{F}$ & - & - & $\mathrm{R}$ & - & - & - & - & - & - & - & $\mathrm{F}$ & - & - & - & - & - & - & A \\
\hline $4 \mathrm{H}-6,60$ & A & G & - & $R$ & - & - & $\mathrm{F}$ & - & - & - & - & - & - & - & $\mathrm{F}$ & - & - & - & - & $\mathrm{R}$ & - & A \\
\hline $4 \mathrm{H}-\mathrm{CC}$ & A & G & - & $F$ & - & - & - & - & $\mathrm{F}$ & - & - & - & - & - & $\mathrm{F}$ & - & - & - & - & $\mathrm{R}$ & - & A \\
\hline $5 \mathrm{H}-1,60$ & A & M & - & $\mathrm{F}$ & - & - & - & - & C & - & - & - & - & - & C & - & - & - & - & $\mathrm{F}$ & - & A \\
\hline $5 \mathrm{H}-2,60$ & A & M & - & $\mathrm{R}$ & - & - & - & - & $\mathrm{F}$ & - & - & - & - & - & A & - & - & - & - & $\mathrm{F}$ & - & A \\
\hline $5 \mathrm{H}-3,60$ & A & G & - & - & $\mathrm{R}$ & - & - & - & C & - & - & - & - & - & C & - & - & - & - & R & - & A \\
\hline $5 \mathrm{H}-4,60$ & A & M & - & $\mathrm{R}$ & A & $\mathrm{R}$ & - & - & A & - & - & - & - & - & $R$ & - & - & - & - & $\mathrm{F}$ & - & A \\
\hline $5 \mathrm{H}-5,60$ & $A$ & $\mathrm{M}$ & - & - & A & - & - & - & C & - & - & - & - & - & $C$ & - & - & - & - & $A$ & - & A \\
\hline $5 \mathrm{H}-6,60$ & A & G & - & $\mathrm{F}$ & A & - & - & - & C & - & 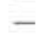 & - & - & - & $\mathrm{F}$ & - & - & 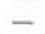 & - & A & - & A \\
\hline SH-CC & C & $M$ & - & $\mathrm{R}$ & C & - & - & - & C & - & - & - & - & - & c & - & - & - & - & A & - & C \\
\hline $6 \mathrm{H}-1,60$ & A & G & - & - & - & - & - & - & - & - & - & - & - & - & $\mathbf{R}$ & - & - & - & - & A & - & $\mathrm{F}$ \\
\hline $6 \mathrm{H}-2,60$ & A & G & - & - & $\mathrm{F}$ & - & - & - & $\mathrm{C}$ & - & - & - & - & - & C & - & - & - & - & A & - & $\mathrm{F}$ \\
\hline $6 \mathrm{H}-3,50$ & A & G & - & - & $\mathrm{R}$ & - & - & - & $\mathrm{F}$ & - & - & - & - & - & $C$ & - & - & - & - & A & - & $R$ \\
\hline $6 \mathrm{H}-4,60$ & A & G & - & & $\mathrm{R}$ & - & - & - & $\mathrm{F}$ & - & - & - & - & - & C & - & - & & - & A & - & $\mathbf{R}$ \\
\hline $6 \mathrm{H}-5,60$ & A & G & - & - & $\mathrm{F}$ & - & - & - & $\mathrm{F}$ & - & - & - & - & - & $\mathrm{F}$ & - & - & - & - & A & - & F \\
\hline $6 \mathrm{H}-6,60$ & A & G & - & - & $\mathrm{F}$ & - & - & - & $\mathrm{F}$ & - & - & - & - & - & C & - & - & - & - & A & - & $\mathbf{R}$ \\
\hline $6 \mathrm{H} \cdot \mathrm{CC}$ & A & G & - & - & $\mathrm{F}$ & - & - & - & $\mathrm{F}$ & - & - & - & - & - & $\mathrm{F}$ & - & - & - & - & A & - & $\mathrm{F}$ \\
\hline $7 \mathrm{H}-1,60$ & A & G & - & - & $\mathrm{F}$ & - & - & - & $\mathrm{F}$ & - & - & - & - & - & $C$ & - & - & - & - & A & - & $\mathrm{F}$ \\
\hline $7 \mathrm{H}-2,60$ & A & G & - & - & $\mathrm{F}$ & - & - & - & $\mathrm{R}$ & - & - & - & - & - & $\mathrm{F}$ & - & - & - & - & A & - & $\mathrm{F}$ \\
\hline $7 \mathrm{H}-3,70$ & A & G & - & - & $\mathrm{R}$ & - & - & - & $\mathrm{R}$ & - & - & - & - & - & - & - & - & - & $\mathrm{F}$ & - & - & C \\
\hline $7 \mathrm{H}-4,70$ & A & M & - & - & $\ddot{A}$ & $\mathbf{R}$ & - & - & - & - & - & - & - & $\mathrm{R}$ & $\mathrm{R}$ & - & - & - & - & - & - & - \\
\hline $7 \mathrm{H}-5,70$ & A & G & - & - & A & A & - & - & $\mathrm{R}$ & - & - & - & - & - & - & - & - & - & $\mathrm{R}$ & - & - & $\mathrm{C}$ \\
\hline $7 \mathrm{H}-6,70$ & C & M & - & - & A & $\mathrm{F}$ & - & - & $R$ & - & - & - & - & - & $\mathrm{R}$ & - & - & - & - & - & - & - \\
\hline $7 \mathrm{H} \cdot \mathrm{CC}$ & A & G & - & & A & c & - & - & - & 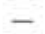 & 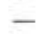 & - & & - & $\mathrm{F}$ & - & - & & - & - & - & - \\
\hline $8 \mathrm{H}-1,60$ & A & G & - & - & A & $\mathrm{F}$ & - & - & $\bar{C}$ & - & - & - & - & - & $\mathrm{F}$ & $=$ & - & - & - & - & - & - \\
\hline $8 \mathrm{H}-2,60$ & A & M & - & - & C & - & - & - & $\mathrm{F}$ & - & - & - & - & $\mathrm{R}$ & $\mathrm{R}$ & - & - & - & $\mathrm{R}$ & - & - & - \\
\hline $8 \mathrm{H}-3,60$ & A & $\mathrm{M}$ & - & - & C & $\mathrm{R}$ & - & - & $\mathrm{R}$ & - & - & - & - & $\mathrm{R}$ & $\mathrm{F}$ & - & - & - & - & - & - & - \\
\hline $8 \mathrm{H}-4,60$ & A & & - & & $F$ & & & & - & - & - & - & & - & C & - & - & - & - & - & - & - \\
\hline $8 \mathrm{H}-5,60$ & A & G & - & - & - & - & - & - & $\mathrm{F}$ & - & - & - & - & $\mathrm{R}$ & $\mathrm{F}$ & - & - & - & - & - & - & - \\
\hline $8 \mathrm{H}-6,60$ & A & G & - & - & - & - & - & - & $\mathrm{R}$ & - & - & - & - & - & C & - & - & $\mathrm{R}$ & - & - & - & - \\
\hline $8 \mathrm{H} \cdot \mathrm{CC}$ & A & G & - & - & - & - & - & - & $\mathrm{F}$ & - & - & $\mathrm{F}$ & - & $\mathrm{R}$ & $\mathrm{F}$ & - & - & $\mathrm{R}$ & $\mathrm{F}$ & - & - & - \\
\hline $9 \mathrm{H}-1,58$ & A & G & - & - & - & - & - & - & $\mathrm{F}$ & - & - & - & - & $\mathrm{R}$ & $\mathrm{F}$ & - & - & $\mathrm{F}$ & A & - & - & - \\
\hline $9 \mathrm{H}-2,58$ & A & G & - & - & - & - & - & - & $R$ & - & - & - & - & - & A & - & - & $\mathrm{R}$ & A & - & - & - \\
\hline $9 \mathrm{H}-3,58$ & $\mathrm{~F}$ & M & - & & - & - & - & - & $=$ & - & $\mathrm{F}$ & - & & - & $\mathrm{F}$ & - & - & - & - & - & - & - \\
\hline $9 \mathrm{H}-4,58$ & C & M & - & - & - & - & - & - & $\mathrm{R}$ & - & $\mathrm{R}$ & - & - & - & $\mathrm{F}$ & - & - & $\mathrm{R}$ & $\mathrm{F}$ & - & - & - \\
\hline $9 \mathrm{H}-5,58$ & $\mathrm{~F}$ & $\mathrm{~F}$ & - & - & - & - & - & - & - & - & - & - & - & - & $\mathrm{R}$ & - & - & - & - & - & - & - \\
\hline $9 \mathrm{H}-6,58$ & $\mathrm{~F}$ & $M$ & - & - & - & - & - & - & - & - & - & - & - & - & $\mathrm{F}$ & - & - & - & - & - & - & - \\
\hline $9 \mathrm{H}-\mathrm{CC}$ & C & M & - & - & - & - & $\mathrm{R}$ & - & - & - & $\mathbf{R}$ & - & - & - & $\mathrm{C}$ & - & - & - & - & - & - & - \\
\hline
\end{tabular}

Note: Abundance: $\mathrm{A}=$ abundant, $\mathrm{C}=$ common, $\mathrm{F}=$ few, $\mathrm{R}=$ rare; preservation: $\mathrm{G}=$ good, $\mathrm{M}=$ moderate, $\mathrm{P}=$ poor; lowercase letters indicate reworking. 


\begin{tabular}{|c|c|c|c|c|c|c|c|c|c|c|c|c|c|c|c|c|c|c|c|c|c|c|c|c|}
\hline 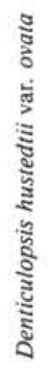 & 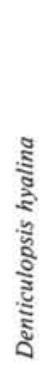 & 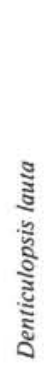 & 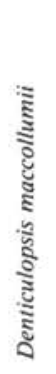 & 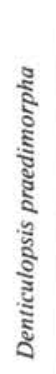 & 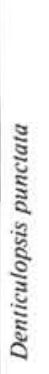 & 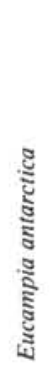 & 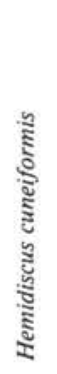 & 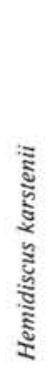 & 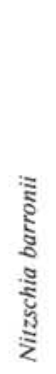 & 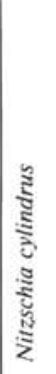 & 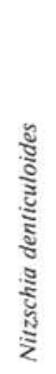 & 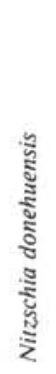 & 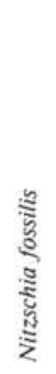 & 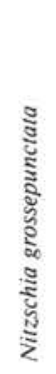 & 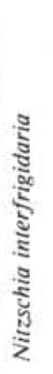 & 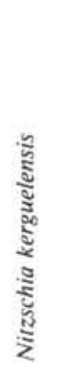 & 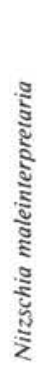 & 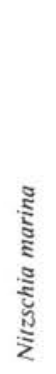 & 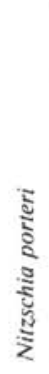 & 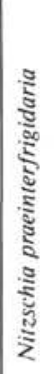 & 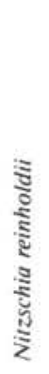 & 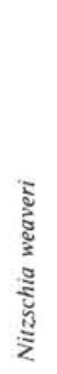 & 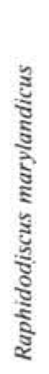 & \\
\hline- & - & - & - & - & - & C & - & - & $F$ & - & - & - & - & - & - & & & - & - & - & & - & & \\
\hline & $\overline{-}$ & $\overline{-}$ & $\overline{-}$ & $\bar{z}$ & - & $\bar{F}$ & $\overline{-}$ & $\overline{\mathrm{F}}$ & $\bar{F}$ & $=$ & $=$ & $=$ & - & - & $\bar{F}$ & C & - & - & - & - & - & - & - & \\
\hline & - & - & - & - & - & - & $=$ & - & $\mathrm{F}$ & - & - & $=$ & $\overline{\mathrm{R}}$ & $=$ & - & - & - & $\overline{\mathrm{R}}$ & - & $\bar{F}$ & $=$ & - & - & \\
\hline- & - & - & - & - & - & - & - & - & R & - & - & - & $\mathrm{F}$ & - & $\mathrm{R}$ & - & - & - & - & R & - & $\mathrm{R}$ & - & - \\
\hline- & $=$ & $=$ & $=$ & $=$ & $\bar{z}$ & $\overline{\bar{F}}$ & $=$ & $=$ & $\mathrm{r}$ & $\bar{c}$ & - & $\overline{\bar{R}}$ & - & - & - & $=$ & $\overline{-}$ & $\overline{\mathrm{R}}$ & $=$ & $\begin{array}{ll}\mathrm{r} \\
\end{array}$ & $\bar{p}$ & - & - & 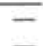 \\
\hline & - & - & - & - & - & - & $\overline{-}$ & $\overline{-}$ & $\overline{-}$ & $\frac{c}{-}$ & - & - & $\overline{-}$ & $=$ & $\overline{-}$ & $\bar{z}$ & $\bar{z}$ & $\begin{array}{l}\mathrm{R} \\
\mathrm{R}\end{array}$ & $\bar{z}$ & $\overline{-}$ & $\stackrel{R}{-}$ & $\bar{z}$ & $\bar{z}$ & \\
\hline $\mathrm{F}$ & $\bar{z}$ & $\bar{z}$ & $\bar{z}$ & $\bar{z}$ & - & $\bar{z}$ & $\bar{z}$ & $\overline{\mathrm{R}}$ & $\bar{z}$ & $\stackrel{\mathrm{R}}{-}$ & $\bar{z}$ & $\underline{\mathrm{R}}$ & $\overline{-}$ & $\bar{z}$ & $\bar{z}$ & $\bar{z}$ & $\bar{z}$ & $\bar{z}$ & $\begin{array}{l}F \\
R\end{array}$ & $\overline{-}$ & $\bar{z}$ & $\bar{z}$ & $\bar{z}$ & - \\
\hline $\mathrm{F}$ & - & - & - & - & - & - & - & - & - & - & - & - & - & - & - & - & - & - & - & - & - & - & - & - \\
\hline $\begin{array}{l}\mathrm{A} \\
\mathrm{F}\end{array}$ & - & - & - & - & & - & - & $\overline{0}$ & - & & & $\overline{0}$ & - & - & 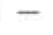 & - & - & - & 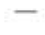 & & - & - & 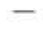 & \\
\hline F & $\bar{z}$ & $\overline{-}$ & $=$ & $\bar{z}$ & & $\bar{R}$ & $=$ & R & $=$ & $z$ & - & $\underline{R}$ & $z$ & $\bar{z}$ & - & - & & $=$ & & - & & - & $\overline{-}$ & \\
\hline A & - & - & - & - & - & - & $R$ & - & - & - & - & - & - & - & - & - & - & - & - & - & - & - & - & - \\
\hline$\underset{R}{R}$ & - & - & - & - & - & - & $\mathrm{R}$ & - & - & - & - & $\mathrm{R}$ & - & - & - & - & - & - & - & - & - & - & - & - \\
\hline $\mathbf{R}$ & - & - & - & - & - & $\mathrm{R}$ & $\mathrm{R}$ & - & - & & - & - & - & - & & - & & - & - & - & 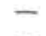 & - & 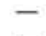 & \\
\hline 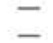 & - & - & - & - & - & 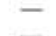 & $p$ & & & & & - & & & & - & & & & & & & & \\
\hline- & - & - & - & $\bar{z}$ & - & $\bar{z}$ & - & - & $\bar{z}$ & 1- & - & $\overline{\mathrm{R}}$ & $\bar{z}$ & $\overline{-}$ & - & $\bar{z}$ & - & $\bar{z}$ & - & $=$ & - & $\bar{z}$ & - & - \\
\hline- & - & - & - & - & - & - & - & - & - & - & - & - & - & - & - & - & - & - & - & - & - & - & - & - \\
\hline & - & & - & - & & 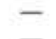 & & - & - & & - & - & - & - & - & - & & - & - & $1-$ & - & - & - & \\
\hline & - & $\overline{\mathrm{C}}$ & - & $\bar{z}$ & & - & - & 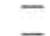 & - & - & - & $\bar{R}$ & $\bar{z}$ & $\bar{z}$ & 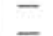 & $\bar{z}$ & & $\bar{z}$ & - & $=$ & & $\bar{z}$ & {[} & \\
\hline - & - & $\mathrm{F}$ & - & - & - & - & - & - & - & - & - & - & - & - & - & - & - & - & - & - & - & - & - & - \\
\hline- & - & $\bar{z}$ & - & - & . & - & - & - & - & - & - & - & - & - & - & - & - & - & - & - & - & - & - & - \\
\hline & 二 & $\mathrm{R}$ & $=$ & - & & 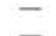 & - & - & - & & . & - & - & - & & - & & - & & - & & & & \\
\hline & 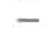 & & & 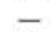 & & - & & & & & & & & & & & & & & & & $=$ & {[} & \\
\hline - & - & - & - & $\mathrm{F}$ & - & - & - & - & - & - & - & - & - & - & - & - & - & - & - & - & - & - & - & - \\
\hline- & - & $\mathrm{F}$ & - & A & - & - & - & - & - & - & - & $\mathrm{A}$ & - & - & - & - & - & - & - & - & - & - & - & - \\
\hline & - & $\mathrm{F}$ & $R$ & A & & 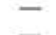 & 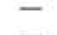 & - & - & & & & & & & - & & & & & & & & \\
\hline & $F$ & 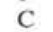 & 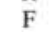 & & & 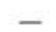 & & 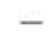 & & & & & & & & & & - & & $=$ & & $=$ & I & \\
\hline - & - & - & - & - & - & - & - & - & - & - & - & - & - & $R$ & - & - & - & - & - & - & - & - & - & $\mathrm{R}$ \\
\hline- & $\mathrm{F}$ & $\mathrm{F}$ & $\mathrm{F}$ & - & - & - & - & - & - & - & - & - & - & $R$ & - & - & - & - & - & - & - & - & - & - \\
\hline & $\bar{c}$ & - & $\underline{F}$ & - & & - & 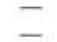 & $\bar{z}$ & $=$ & I & $k$ & F & & & & $\overline{-}$ & & $\overline{-}$ & I & $\overline{-}$ & & $\overline{-}$ & & $\mathrm{R}$ \\
\hline & $\mathrm{R}$ & - & $C$ & 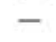 & & 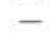 & & - & 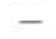 & 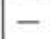 & 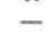 & - & & 1 & 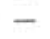 & 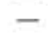 & & $\pi$ & & 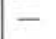 & & - & - & \\
\hline & & & & - & & - & & - & & - & & & & & & & & & & & & & - & $\mathrm{F}$ \\
\hline & - & - & A & - & - & - & - & - & - & - & - & - & - & $\mathbf{F}$ & - & - & - & - & - & - & - & - & - & $F$ \\
\hline & 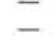 & $\mathrm{R}$ & A & 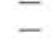 & & - & & 7 & & & & & & & & & & & & - & & & & \\
\hline & & $P$ & 0 & 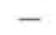 & $\mathrm{F}$ & 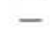 & & 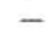 & & & & & & & & & & & & & & & & \\
\hline- & - & - & - & - & - & - & - & - & - & - & - & - & - & - & - & - & $\mathrm{F}$ & - & - & - & - & - & $\mathrm{F}$ & - \\
\hline- & - & - & - & - & - & - & - & - & - & - & - & - & - & - & - & - & & & - & & - & - & - & - \\
\hline & $=$ & - & - & $=$ & & $\bar{z}$ & $=$ & $\bar{z}$ & $\bar{z}$ & - & $\overline{-}$ & 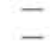 & $=$ & $\bar{z}$ & $\bar{z}$ & $\bar{z}$ & $\begin{array}{l}\mathrm{F} \\
\mathrm{F}\end{array}$ & $\bar{z}$ & & $=$ & & - & & - \\
\hline & - & - & 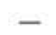 & - & - & - & - & - & - & - & - & - & - & - & - & - & $\mathrm{R}$ & & & - & & - & & \\
\hline & 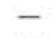 & - & - & - & 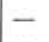 & 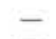 & 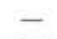 & - & 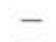 & 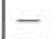 & 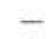 & 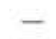 & - & - & 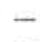 & 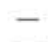 & $\pi$ & - & & - & & 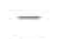 & & D \\
\hline & - & - & - & & & - & & - & & $1-$ & & - & & & & & & & & & & & & \\
\hline
\end{tabular}


Table 8 (Continued).

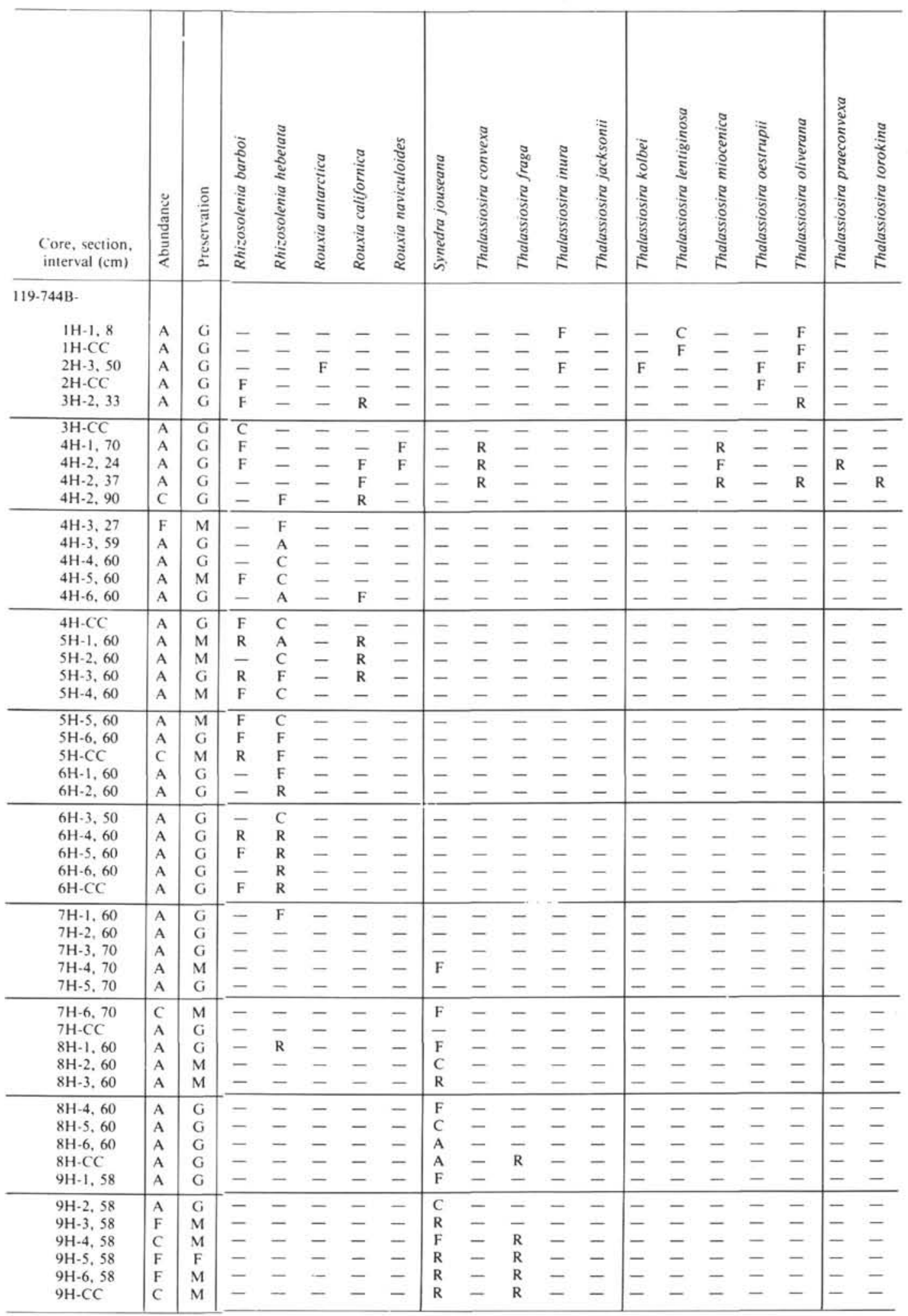




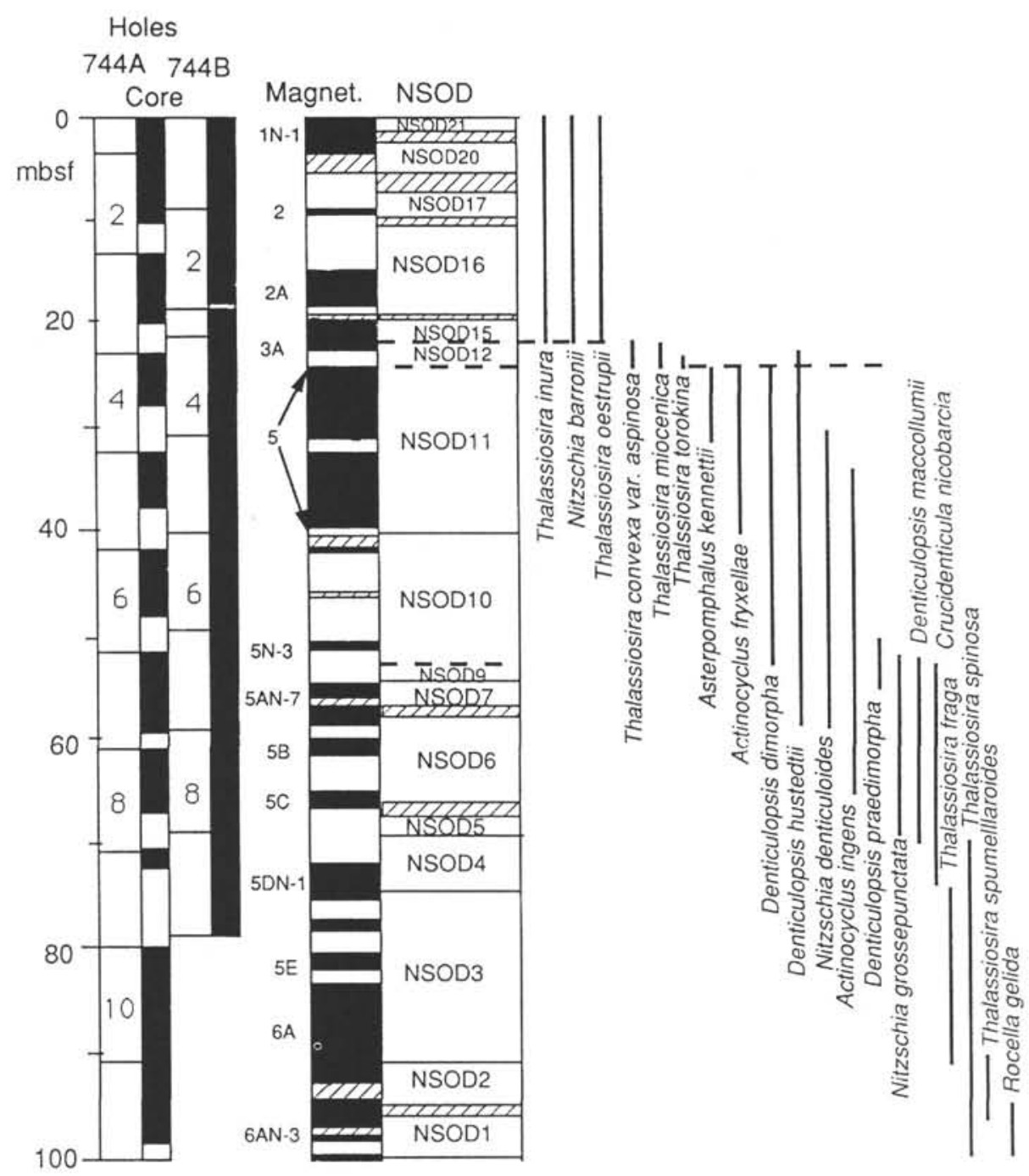

Figure 4. Stratigraphic recovery (Barron, Larsen, et al., 1989), paleomagnetics (H. Sakai, pers. comm., 1989), assigned zones, and the range of selected species for the upper portion of Hole 744A and Hole 744B. The width of a range bar indicates relative abundance (see tables for specifics).

this zone (Sample 119-744A-13H-2, $60 \mathrm{~cm}$ ) and the last occurrences of Rouxia granda, Asterolampra schmidtii, and Hemiaulus insignis also occur in this interval.

The Rhizosolenia oligocenica Zone extends from Sample 119$744 \mathrm{~A}-14 \mathrm{H}-2,60-62 \mathrm{~cm}$, to the base of Core $119-744 \mathrm{~A}-16 \mathrm{H}$. Gombos and Ciesielski (1983) previously defined a Rhizosolenia gravida Zone as the interval from the last $R$. gravida (synonym with $R$. oligocenica) down to the first occurrence of Asteromphalus oligocenicus. This zone is redefined here because of the inconsistent occurrence of A. oligocenicus. Although the last occurrence of $R$. oligocenica is a useful secondary indicator and is used here to mark the $a / b$ subzone boundary, the usefulness of this event for broad-scale correlation still needs to be tested (see Baldauf and Monjanel, 1989, for discussion). Instead, the top of the $R$. oligocenica Zone, as used here, is extended and defined by the first occurrence of $R$. vigilans. The first occurrence of $S$. jouseana lies in the middle portion of subzone b; unfortunately, the paleomagnetic signature from this interval disallows calibration of this event to the magnetostratigraphy. Other species prevalent through the $R$. oligocenica Zone include Cestodiscus reticulatus, Rouxia obesa, Rouxia granda, and Coscinodiscus hajosiae. Hemiaulus caracteristicus occurs in the lowermost sample of Core 119-744A-16H. The decline in diatom abundance and preservation below Core 119-744A-16H limits the usefulness of diatoms for stratigraphic purposes.

\section{Site 745}

Site 745 is a deep-water $(4082.5 \mathrm{~m})$ site positioned on the southern end of the Kerguelen Plateau (Table 1 and Fig. 1). The two holes cored at this site recovered a nearly continuous sequence of uppermost Miocene to Quaternary sediments consisting predominately of diatom ooze and diatom clay. Diatoms are generally common and moderately to well preserved in all samples examined from this sequence.

Table 9 lists the occurrence of selected and stratigraphically important diatoms in Hole 745B. Diatoms ranging from the latest Quaternary NSOD Zone 21 through the latest Miocene NSOD Zone 11 were recovered from Hole 745B. The downhole magnetic polarity logs for Hole 745B (H. Sakai, pers. comm., 1989) are shown in Figure 6 along with the ranges of stratigraphically important diatom species. Comparison of these diatom ranges with published ranges (see subsequent discussion) and compari- 


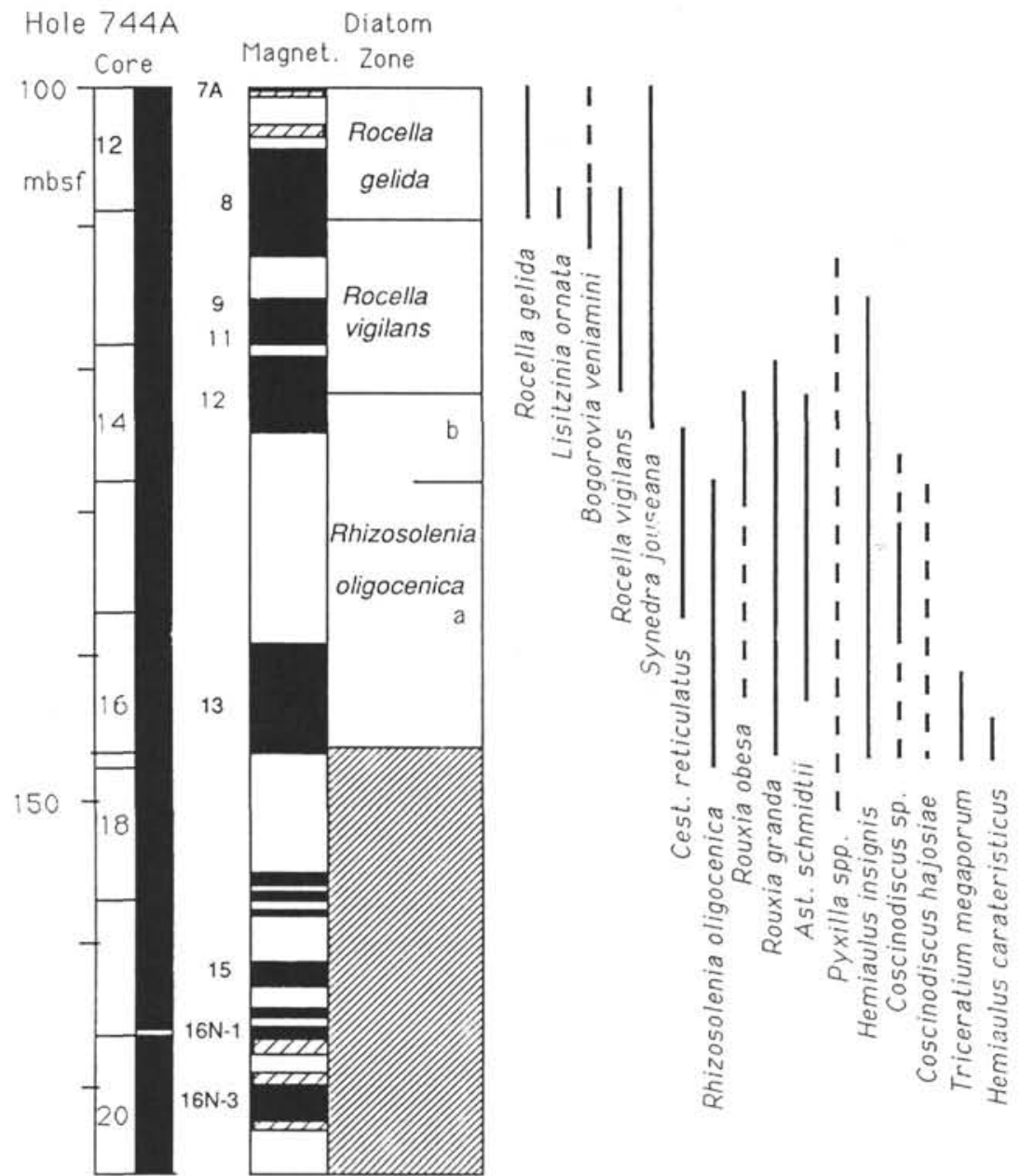

Figure 5. Stratigraphic recovery (Barron, Larsen, et al., 1989), paleomagnetics (H. Sakai, pers. comm., 1989), assigned zones, and the range of selected species from the lower portion of Hole $744 \mathrm{~A}$. The width of a range bar indicates relative abundance (see tables for specifics), and dashes indicate sporadic occurrences of an individual species for a given stratigraphic interval. Ruled area at base of diatom zone column indicates an unzoned interval.

son of the downhole magnetic polarity log with the Berggren et al. (1985) magnetic polarity time scale suggest an identification of the magnetic polarity events in Hole 745B (Fig. 6). A sequence ranging from the Brunhes magnetic polarity Chron to the lower normal event of Chron C3A (5.68-5.89 Ma) was cored at Site 745. This expanded section $(215 \mathrm{~m})$ represents an excellent Quaternary to Miocene deep-water reference section for the Southern Ocean.

The first three cores from Hole 745B are assigned to NSOD Zone 21 based on the last occurrence of Actinocyclus ingens in Sample 119-744B-4H-CC. The diatom assemblage in this interval is dominated by specimens of Nitzschia kerguelensis and Thalassiothrix longissima.

Rhizosolenia barboi has a consistent occurrence in the upper part of its stratigraphic range at Site 745 . The last occurrence of this species is placed in Sample 119-745B-10H-CC, which allows the assignment of the interval from Core 119-745B-5H to the lower part of Core 119-745B-10H to NSOD Zone 20. The last occurrence of Coscinodiscus elliptopora falls within this interval, in Sample 119-745B-6H-CC.

NSOD Zone 19 extends from Sample 119-745A-10H-CC to immediately above Sample 119-745B-11H-3, 60-62 cm. The last occurrence of Thalassiosira kolbei, which marks the base of this zone, is placed in this latter sample. This event is calibrated to magnetic polarity event $\mathrm{C} 2 \mathrm{~N}-1$ (Fig. 6) and has an estimated age of 1.75-1.9 Ma at Site 745. The last occurrence of Coscinodiscus vulnificus, which delineates the base of the underlying NSOD Zone 18, is placed between Samples 119-745B-12H-2, 60-62 cm, and 119-745B-12H-4, $60-62 \mathrm{~cm}$. The last occurrence of $C$. vulnificus correlates with Subchron C2R-1 (2.2-2.3 Ma). These zones are characterized by Actinocyclus ingens, Eucampia antarctica, Nitzschia barronii, and Thalassionema nitzschioides.

NSOD Zone 17 has a base defined by the last occurrence of Cosmiodiscus insignis in Sample 119-745B-12H-2, 60-62 cm. This sample correlates with the lowermost portion of Chron $\mathrm{C} 2$, suggesting an estimated age of 2.4-2.45 Ma for this event.

NSOD Zone 16 extends from Sample 119-745B-12H-2, 60-62 $\mathrm{cm}$, to immediately above the first occurrence of Nitzschia interfrigidaria in Sample 119-745B-15H-5, 60-62 cm. The last occurrence of Nitzschia weaveri in Sample 119-745B-14H-4, 64-66 $\mathrm{cm}$, occurs within this zone.

NSOD Zone 15 extends from Samples 119-745B-15H-6, 60$62 \mathrm{~cm}$, to $119-745 \mathrm{~B}-17 \mathrm{H}-4,60-62 \mathrm{~cm}$. This zone is characterized 
by an abrupt increase in specimens of Nitzschia barronii. The first occurrence of $N$. barronii correlates with magnetic polarity event C3R-1 and has an estimated age of $4.0 \mathrm{Ma}$.

NSOD Zone 14 extends from immediately below the first occurrence of Nitzschia barronii in Sample 119-745B-17H-4, 60-62 cm, to the first occurrence of Thalassiosira inura in Sample $119-745 \mathrm{~B}-20 \mathrm{H}-1,60-62 \mathrm{~cm}$. The base of this zone correlates with the fourth reversed event of the Gilbert magnetic polarity Chron (C3R-4) and has an estimated age of 4.7-4.8 Ma.

The first occurrence of Thalassiosira oestrupii in Sample 119-745B-21H-2, 60-62 cm, allows placement of the base of NSOD Zone 13 at this depth. Calibration of this event to the paleomagnetic stratigraphy suggests an age of 5.1-5.3 Ma, which is an age similar to that determined by Baldauf (1985) for the eastern equatorial Pacific.

NSOD Zone 12 extends from immediately below the occurrence of Thalassiosira inura in Sample 119-745B-20H-1, 60-62 $\mathrm{cm}$, to the base of Hole 745B. The base of Hole 745B has an extrapolated age of $\sim 5.8 \mathrm{Ma}$.

\section{Site 746}

Site 746 is a companion site to Site 745 (Table 1 and Fig. 1). One hole was drilled to a depth of $280.8 \mathrm{mbsf}$ with recovery of a stratigraphic sequence of upper Miocene and lower Pliocene diatomaceous ooze and clays. Diatoms are present in the samples examined and are generally abundant and moderately to well preserved. The occurrence of common and stratigraphically important diatoms in the continuously cored interval from Cores $119-746 \mathrm{~A}-4 \mathrm{H}$ through $119-746 \mathrm{~A}-16 \mathrm{H}$ (164.8-280.8 mbsf) is shown in Table 10. Downhole magnetic polarity logs (Keating, this volume) and the ranges of stratigraphically significant diatom species are shown in Figure 7. Comparison of published age estimates of diatom datums with the downhole sequence of normal and reversed polarity events and the magnetic polarity time scale of Berggren et al. (1985) suggests that the recovered section ranges from the lower normal event of Chron $\mathrm{C} 3 \mathrm{~A}$ to the upper normal event of Chron C5 (Fig. 7).

The upper part of Core 119-746A-4H (164.8 to about 169 mbsf) appears to be correlative to the lower part of Core 119$745 \mathrm{~B}-24 \mathrm{H}$ (about 208 to $215.1 \mathrm{mbsf}$ ), as both are correlated with the lower normal event of Chron C3A (Figs. 6 and 7) and Thalassiosira praeconvexa is recorded from both intervals (Tables 9 and $10)$.

Diatoms recovered from this stratigraphic sequence (Cores 119-746A-4H through 110-746A-16H) can be placed into NSOD Zone 12 and underlying NSOD Zone 11 . This expanded section offers an excellent upper Miocene reference section for the interval ranging in age from about 5.8 to $9.8 \mathrm{Ma}$, based on the presence of Thalassiosira praeconvexa (last occurrence $5.8 \mathrm{Ma}$ ) in Sample 119-746A-4H-1, 60-62 cm, and the absence of common Denticulopsis dimorpha $(9.9 \mathrm{Ma})$ in Sample 119-746A-16H-CC, at the base of the hole. No hiatuses are apparent.

Of particular interest at Site 746 is the occurrence of species such as Nitzschia miocenica, Thalassiosira praeconvexa, and Thalassiosira miocenica that are typical of the lower latitudes. Their presence at Site 746 (as well as other Leg 119 sites) during the late Miocene suggests that the Antarctic Polar Front was south of this site during this time. Calibration of these events to the magnetic stratigraphy (H. Sakai, pers. comm., 1989) allows age estimates of $5.8 \mathrm{Ma}$ (first occurrence of $T$. miocenica), $5.8 \mathrm{Ma}$ (first occurrence of Thalassiosira convexa var. aspinosa), and 6.1 $\mathrm{Ma}$ (first occurrence of Nitzschia miocenica) and suggests that the younger two events in the Kerguelen Plateau region are synchronous with those in the low latitudes.

Within NSOD Zone 12 the warm to warm-temperate diatoms Thalassiosira miocenica and $T$. convexa var. aspinosa have their first occurrences in Sample 119-746A-4H-1, 60-62 cm, whereas T. praeconvexa and Nitzschia miocenica first occur in Samples 119-746A-4H-4, 60-62 cm, and 119-746A-4H-6, 60-62 cm, respectively. Together, these diatoms suggest that the normal polarity event identified in the upper part of Core 119-746A-4H (Fig. 7) represents the lower normal event of Chron $\mathrm{C} 3 \mathrm{~A}$, whereas their presence at $59^{\circ} \mathrm{S}$ suggests a period of relatively warm paleotemperatures between about 6.1 and $5.9 \mathrm{Ma}$.

Other significant diatom datum levels within the upper portion of NSOD Zone 12 in Hole 746A include the first occurrence of Thalassiosira jacksonii (estimated age 6.1 Ma) in Sample 119$746 \mathrm{~A}-4 \mathrm{H}-5,60-62 \mathrm{~cm}$, the last common occurrence of Denticulopsis hustedtii $(6.4 \mathrm{Ma})$ in Sample 119-746A-5H-4, 60-62 cm, and the last occurrence of Actinocyclus ingens var. ovalis (6.5 Ma) in Sample 119-746A-6H-1, 65-67 cm (Fig. 7). Within the lower portion of NSOD Zone 12 the last occurrence of Thalassiosira gersondei $\mathrm{n}$. sp. (about 7.2 Ma) is recognized in Sample 119$746 \mathrm{~A}-7 \mathrm{H}-5,60-62 \mathrm{~cm}$, the first occurrence of A. ingens var. ovalis $(7.3 \mathrm{Ma})$ is placed in Sample $119-746 \mathrm{~A}-8 \mathrm{H}-2,60-62 \mathrm{~cm}$, and the first occurrence of Nitzschia cylindrica ( $7.4 \mathrm{Ma})$ lies in Sample 119-746A-8H-3, 60-62 cm (Fig. 7). The latter diatom datum identifies the three normal events recorded in Hole 746A by H. Sakai (pers. comm., 1989) between 189 and 207 mbsf as magnetic anomaly 4.

The first occurrence of Thalassiosira torokina in Sample 119. 746 A9HCC marks the base of NSOD Zone 12. This event lies in the reversed interval between magnetic anomalies 4 and $4 \mathrm{~A}$, which is similar to its placement in Hole $689 \mathrm{~B}$ by Gersonde and Burckle (1990). Immediately below this horizon the first occurrences of Cosmiodiscus intersectus and Thalassiosira mahoodii are identified in Sample 119-746A-10H-2, 60-62 cm (7.9 Ma; Fig. 7).

Within NSOD Zone 11, the last consistent occurrence of Denticulopsis dimorpha is tentatively identified in Sample 119-746A$11 \mathrm{H}-1,60-62 \mathrm{~cm}$, (about $8.0 \mathrm{Ma}$; Fig. 7); however, D. dimorpha is commonly reworked upsection (note occurrences in Cores $119-746 \mathrm{~A}-4 \mathrm{H}$ and $119-746 \mathrm{~A}-5 \mathrm{H}$; Table 10 ), and the reader is cautioned against using this datum level for correlation in the Southern Ocean.

Isolated occurrences of Denticulopsis dimorpha var. areolata are recorded in Samples 119-746A-11H-6, 60-62 cm, and 119$746 \mathrm{~A}-11 \mathrm{H}-\mathrm{CC}$ (Table 10), at a level (approximately 8.4-8.7 Ma) within the middle portion of NSOD Zone 11 similar to that found in Hole 744B (Table 8). At about the same level (Samples 119$746 \mathrm{~A}-11 \mathrm{H}-5,60-62 \mathrm{~cm}$, and $119-746 \mathrm{~A}-11 \mathrm{H}-6,60-62 \mathrm{~cm})$, rare occurrences of Thalassiosira burckliana were tabulated, which supports the correlation of the normal polarity events found by $\mathrm{H}$. Sakai (pers. comm., 1989) between 228 and 235 mbsf (Core $119-746 \mathrm{~A}-11 \mathrm{H})$ with anomaly $4 \mathrm{~A}$ (Barron, in press).

Asteromphalus kennettii can be identified from Samples 119$746 \mathrm{~A}-13 \mathrm{H}-1,60-62 \mathrm{~cm}$, through $119-746 \mathrm{~A}-14 \mathrm{H}-1,60-62 \mathrm{~cm}$. Specimens are typically dissolved, attesting to the fragile nature of this species and arguing against its use as a zonal marker species in the Southern Ocean. If the lack of A. kennettii below Sample 119-746A-14H-1, 60-62 cm, is not due to dissolution, Gersonde and Burckle's (1990) stratigraphy suggests that an age of $9.6 \mathrm{Ma}$ can be assigned to the interval between the recovered portions of Cores 119-746A-14H and 119-746A-15H (253.7$261.5 \mathrm{mbsf}$ ).

The lowermost datum level recorded in Hole 746A is the first occurrence of Thalassiosira gersondei in Sample 119-746A-15H$1,56-58 \mathrm{~cm}$. Because the last common occurrence of Denticulopsis dimorpha (9.9 Ma; Gersonde and Burckle, 1990) and first occurrence of Actinocyclus fryxellae (10.5 Ma; Fig. 4) were not reached in Hole $746 \mathrm{~A}$, the first occurrence of $T$. gersondei is estimated at 9.7-9.8 Ma. 
Table 9. Occurrence of selected diatom species observed from Hole 745B.

\begin{tabular}{|c|c|c|c|c|c|c|c|c|c|c|c|c|c|c|c|c|c|c|c|c|c|c|}
\hline $\begin{array}{l}\text { Core, section, } \\
\text { interval }(\mathrm{cm})\end{array}$ & 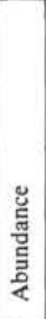 & 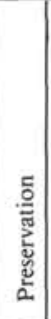 & 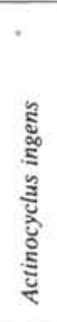 & 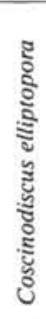 & 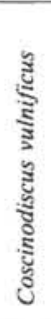 & 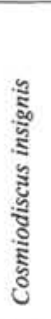 & 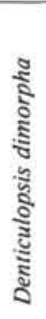 & 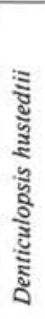 & 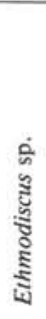 & 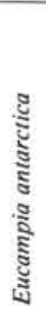 & 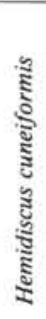 & 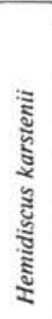 & 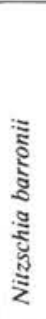 & 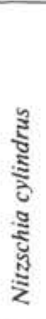 & 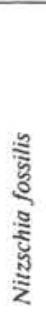 & 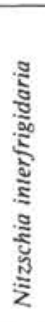 & 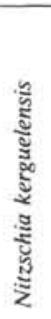 & 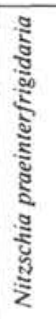 & 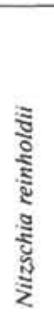 & 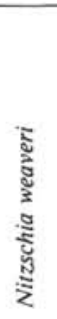 & 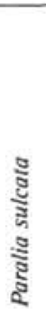 & 高 \\
\hline \multicolumn{23}{|l|}{$119-745 \mathrm{~B}-$} \\
\hline $1 \mathrm{H}-\mathrm{CC}$ & $\begin{array}{l}\mathrm{C} \\
\mathrm{A} \\
\mathrm{A} \\
\mathrm{A} \\
\mathrm{A}\end{array}$ & $\begin{array}{l}\mathrm{M} \\
\mathrm{M} \\
\mathrm{G} \\
\mathrm{M} \\
\mathrm{M}\end{array}$ & $\frac{\bar{r}}{\bar{r}}$ & $\begin{array}{l}\bar{z} \\
\bar{z}\end{array}$ & $\begin{array}{l}\bar{z} \\
\bar{z} \\
\bar{z}\end{array}$ & $\begin{array}{l}\overline{-} \\
\overline{-} \\
\overline{-}\end{array}$ & $\begin{array}{l}- \\
\overline{-} \\
\bar{R}\end{array}$ & $\begin{array}{l}\mathrm{R} \\
- \\
- \\
\bar{R}\end{array}$ & $\begin{array}{l}\bar{z} \\
\bar{z} \\
\overline{-}\end{array}$ & $\begin{array}{l}\mathrm{R} \\
\mathrm{R} \\
\mathrm{R} \\
\mathrm{R} \\
\mathrm{R}\end{array}$ & $\begin{array}{l}\bar{z} \\
\overline{-} \\
\overline{-}\end{array}$ & $\begin{array}{l}\overline{-} \\
\bar{R} \\
-\end{array}$ & $\begin{array}{l}\mathrm{R} \\
\mathrm{R} \\
\mathrm{F} \\
\mathrm{R} \\
\mathrm{R}\end{array}$ & $\begin{array}{l}\bar{z} \\
\overline{-} \\
\overline{-}\end{array}$ & $\begin{array}{l}\bar{z} \\
\bar{z} \\
\overline{-}\end{array}$ & $\begin{array}{l}\bar{z} \\
\bar{z}\end{array}$ & $\begin{array}{l}\text { F } \\
\text { C } \\
\text { A } \\
\text { F } \\
\text { F }\end{array}$ & $\begin{array}{l}\bar{z} \\
\bar{z} \\
-\end{array}$ & $\begin{array}{l}\bar{z} \\
\overline{-}\end{array}$ & $\begin{array}{l}\bar{z} \\
\overline{-} \\
\overline{-}\end{array}$ & $\begin{array}{l}\mathrm{R} \\
\mathrm{R} \\
\mathrm{R} \\
- \\
-\end{array}$ & $\begin{array}{l}\bar{z} \\
\bar{z}\end{array}$ \\
\hline $\begin{array}{l}9 \mathrm{H}-\mathrm{CC} \\
10 \mathrm{H}-\mathrm{CC}\end{array}$ & $\begin{array}{l}\mathrm{A} \\
\mathrm{A} \\
\mathrm{C}\end{array}$ & $\begin{array}{l}\mathrm{M} \\
\mathrm{M} \\
\mathrm{M} \\
\mathrm{M} \\
\mathrm{M}\end{array}$ & $\begin{array}{l}\mathrm{R} \\
\mathrm{F} \\
\mathrm{C} \\
\mathrm{A} \\
\mathrm{C}\end{array}$ & $\begin{array}{l}R \\
R \\
R \\
R \\
-\end{array}$ & $\begin{array}{l}\bar{z} \\
\bar{z} \\
\bar{z}\end{array}$ & $\begin{array}{l}\bar{z} \\
\bar{z} \\
\bar{r}\end{array}$ & $\begin{array}{l}\bar{z} \\
\overline{-}\end{array}$ & $\begin{array}{l}\bar{z} \\
\overline{-} \\
\overline{\mathrm{R}}\end{array}$ & $\begin{array}{l}\overline{-} \\
\bar{R} \\
\overline{-}\end{array}$ & $\begin{array}{l}\mathrm{R} \\
\mathrm{R} \\
\mathrm{R} \\
- \\
-\end{array}$ & $\begin{array}{l}\overline{-} \\
\overline{-} \\
\bar{R}\end{array}$ & $\begin{array}{l}\bar{z} \\
\bar{z} \\
\bar{z}\end{array}$ & $\mathrm{C}$ & $\begin{array}{l}\bar{z} \\
\bar{z} \\
\bar{z}\end{array}$ & $\begin{array}{l}\bar{z} \\
\bar{z}\end{array}$ & $\begin{array}{l}\bar{z} \\
\bar{z}\end{array}$ & $\begin{array}{l}\mathrm{R} \\
\mathrm{R} \\
\mathrm{R} \\
\mathrm{R} \\
\mathrm{R}\end{array}$ & $\begin{array}{l}\bar{z} \\
\bar{z} \\
\overline{-}\end{array}$ & $\begin{array}{l}\overline{-} \\
\overline{-} \\
\overline{-}\end{array}$ & $\begin{array}{l}\overline{-} \\
\overline{-} \\
\overline{-}\end{array}$ & $\begin{array}{l}\bar{R} \\
R \\
R \\
R\end{array}$ & $\begin{array}{l}\bar{z} \\
\bar{z} \\
\bar{z}\end{array}$ \\
\hline & $\begin{array}{l}\mathrm{C} \\
\mathrm{C} \\
\mathrm{R} \\
\mathrm{F} \\
\mathrm{F}\end{array}$ & $\begin{array}{l}\mathrm{M} \\
\mathrm{M} \\
\mathrm{P} \\
\mathrm{P} \\
\mathrm{M}\end{array}$ & $\begin{array}{l}\mathrm{C} \\
\mathrm{F} \\
\mathrm{R} \\
\mathrm{R} \\
-\end{array}$ & $\begin{array}{l}\bar{z} \\
\bar{z} \\
\bar{z}\end{array}$ & $\begin{array}{l}\overline{-} \\
\overline{-} \\
\overline{\mathrm{R}}\end{array}$ & $\begin{array}{l}\overline{-} \\
\bar{R} \\
\overline{-}\end{array}$ & $\begin{array}{l}\bar{z} \\
\bar{z}\end{array}$ & $\begin{array}{l}\bar{z} \\
\bar{z} \\
\overline{-}\end{array}$ & $\begin{array}{l}\bar{z} \\
\bar{z} \\
\overline{-}\end{array}$ & $\begin{array}{l}\bar{R} \\
\bar{F} \\
F\end{array}$ & $\begin{array}{l}\overline{-} \\
\overline{-} \\
\overline{-}\end{array}$ & $\begin{array}{l}\bar{z} \\
\bar{z} \\
\bar{z}\end{array}$ & C & $\begin{array}{l}\bar{z} \\
\bar{z} \\
\overline{-}\end{array}$ & $\begin{array}{l}\bar{z} \\
\bar{z} \\
\bar{z}\end{array}$ & $\begin{array}{l}\bar{R} \\
R \\
R \\
F\end{array}$ & $\begin{array}{l}\mathrm{R} \\
\mathrm{R} \\
- \\
- \\
-\end{array}$ & $\begin{array}{l}\bar{z} \\
\bar{z} \\
\overline{-}\end{array}$ & $\begin{array}{l}\bar{I} \\
\bar{I} \\
\overline{-}\end{array}$ & $\begin{array}{l}\bar{R} \\
R\end{array}$ & $\begin{array}{l}\mathrm{R} \\
\mathrm{R} \\
\mathrm{R} \\
\mathrm{F} \\
\mathrm{R}\end{array}$ & $\begin{array}{l}\bar{z} \\
\bar{z} \\
\overline{-}\end{array}$ \\
\hline $\begin{array}{l}13 \mathrm{H}-7,60-62 \\
14 \mathrm{H}-1,60-62\end{array}$ & $\begin{array}{l}\mathrm{C} \\
\mathrm{A} \\
\mathrm{F}\end{array}$ & $\begin{array}{l}\mathrm{M} \\
\mathrm{P} \\
\mathrm{M} \\
\mathrm{M} \\
\mathrm{M}\end{array}$ & $\begin{array}{l}\bar{I} \\
\overline{ }\end{array}$ & $\begin{array}{l}\bar{z} \\
\bar{z} \\
\overline{-}\end{array}$ & $\begin{array}{l}\mathrm{R} \\
\mathrm{F} \\
\mathrm{R} \\
- \\
-\end{array}$ & $\bar{R}$ & $\begin{array}{l}\bar{z} \\
\bar{z}\end{array}$ & $\begin{array}{l}- \\
-\end{array}$ & $\begin{array}{l}\bar{z} \\
\bar{z} \\
\overline{-}\end{array}$ & $\begin{array}{l}\mathrm{R} \\
\mathrm{R} \\
\mathrm{R} \\
\mathrm{R} \\
\mathrm{R}\end{array}$ & $\bar{z}$ & $\begin{array}{l}\bar{z} \\
\bar{z} \\
\bar{z}\end{array}$ & $\mathrm{~K}$ & $\begin{array}{l}\mathrm{R} \\
\overline{-} \\
-\end{array}$ & $\begin{array}{l}\bar{z} \\
\bar{z}\end{array}$ & $\begin{array}{l}- \\
\bar{R} \\
R \\
R\end{array}$ & $\begin{array}{l}\bar{z} \\
\bar{z} \\
\bar{z}\end{array}$ & $\begin{array}{l}\overline{-} \\
\overline{-} \\
\bar{F} \\
\mathrm{R}\end{array}$ & $\begin{array}{l}\overline{-} \\
\bar{R} \\
\overline{-}\end{array}$ & $\begin{array}{l}\mathrm{R} \\
\vec{F} \\
F \\
R\end{array}$ & $\begin{array}{l}R \\
R \\
R \\
- \\
-\end{array}$ & $\begin{array}{l}\bar{z} \\
\overline{-} \\
\overline{-}\end{array}$ \\
\hline $\begin{array}{l}14 \mathrm{H}-2,64-66 \\
14 \mathrm{H}-4,64-66 \\
14 \mathrm{H}-5,64-66\end{array}$ & $\begin{array}{l}\mathrm{F} \\
\mathrm{A} \\
\mathrm{F} \\
\mathrm{A} \\
\mathrm{C}\end{array}$ & \begin{tabular}{l|}
$\mathrm{P}$ \\
$\mathrm{M}$ \\
$\mathrm{P}$ \\
$\mathrm{M}$ \\
$\mathrm{M}$
\end{tabular} & $\begin{array}{l}\bar{I} \\
\bar{I} \\
-\end{array}$ & $\begin{array}{l}\bar{z} \\
\bar{z} \\
\overline{-}\end{array}$ & $\begin{array}{l}\bar{z} \\
\bar{z} \\
\bar{z}\end{array}$ & - & $\begin{array}{l}\bar{z} \\
\bar{z} \\
\overline{-}\end{array}$ & $\begin{array}{l}\frac{\mathrm{R}}{\mathrm{R}} \\
\frac{\mathrm{R}}{\mathrm{R}}\end{array}$ & $\begin{array}{l}\bar{z} \\
\bar{z} \\
\overline{-}\end{array}$ & $\begin{array}{l}\mathrm{R} \\
\mathrm{R} \\
\mathrm{R} \\
\mathrm{R} \\
\mathrm{R}\end{array}$ & $\bar{z}$ & $\begin{array}{l}\bar{z} \\
\bar{z} \\
\bar{z}\end{array}$ & & $\begin{array}{l}\bar{z} \\
\bar{z} \\
\overline{-}\end{array}$ & $\begin{array}{l}\bar{z} \\
\bar{z} \\
\bar{z}\end{array}$ & $\begin{array}{l}\mathrm{R} \\
\mathrm{R} \\
-\end{array}$ & $\begin{array}{l}\bar{z} \\
\bar{z} \\
\overline{-}\end{array}$ & $\begin{array}{l}\frac{\mathrm{R}}{\mathrm{R}} \\
\frac{-}{-}\end{array}$ & $\begin{array}{l}\bar{z} \\
\bar{z} \\
\overline{-}\end{array}$ & $\begin{array}{l}\mathrm{R} \\
\mathrm{R} \\
-\end{array}$ & $\begin{array}{l}R \\
R \\
R\end{array}$ & $\begin{array}{l}\overline{\mathrm{R}} \\
\overline{\mathrm{R}} \\
-\end{array}$ \\
\hline & $\begin{array}{l}\mathrm{A} \\
\mathrm{A}\end{array}$ & $\begin{array}{l}\mathrm{P} \\
\mathrm{P} \\
\mathrm{M} \\
\mathrm{M} \\
\mathrm{M}\end{array}$ & $\begin{array}{l}\bar{I} \\
\bar{I} \\
\overline{-}\end{array}$ & $\bar{z}$ & $\begin{array}{l}\bar{z} \\
\bar{z} \\
\bar{z}\end{array}$ & $\overline{-}$ & $\begin{array}{l}\bar{z} \\
\bar{z} \\
\overline{-}\end{array}$ & $\mathrm{R}$ & $\begin{array}{l}\overline{-} \\
\bar{R} \\
- \\
-\end{array}$ & $\begin{array}{l}\mathrm{R} \\
\mathrm{R} \\
\\
\overline{\mathrm{R}}\end{array}$ & $\bar{z}$ & $\begin{array}{l}\bar{R} \\
\mathrm{R} \\
- \\
-\end{array}$ & $\mathrm{F}$ & $\overline{-}$ & $\begin{array}{l}\bar{z} \\
\bar{z} \\
\bar{z}\end{array}$ & $\begin{array}{l}\mathrm{R} \\
\mathrm{R} \\
-\end{array}$ & $\begin{array}{l}\bar{z} \\
\bar{z} \\
\overline{-}\end{array}$ & $\begin{array}{l}\bar{R} \\
F \\
R \\
F\end{array}$ & $\begin{array}{l}\overline{\mathrm{R}} \\
\overline{\mathrm{R}} \\
\overline{\mathrm{R}}\end{array}$ & $\begin{array}{l}\overline{-} \\
\overline{-} \\
-\end{array}$ & $\frac{\frac{\mathrm{R}}{\mathrm{R}}}{\frac{\mathrm{R}}{\mathrm{R}}}$ & $\begin{array}{l}\overline{-} \\
\overline{-} \\
-\end{array}$ \\
\hline & $\begin{array}{l}\mathrm{A} \\
\mathrm{A}\end{array}$ & M & $\begin{array}{l}\bar{I} \\
\bar{I} \\
\overline{-}\end{array}$ & $\begin{array}{l}\bar{z} \\
\bar{z} \\
\bar{z}\end{array}$ & $\begin{array}{l}\bar{z} \\
\bar{z} \\
\bar{z}\end{array}$ & $\bar{z}$ & $\begin{array}{l}\bar{z} \\
\overline{-} \\
\overline{-}\end{array}$ & $\mathbf{R}$ & $\begin{array}{l}\overline{-} \\
\bar{R} \\
\bar{R}\end{array}$ & $\frac{\mathrm{R}}{\mathrm{R}}$ & $\bar{z}$ & $\begin{array}{l}\bar{z} \\
\bar{z} \\
\bar{R}\end{array}$ & - & $\frac{R}{p}$ & $\begin{array}{l}\overline{\mathrm{R}} \\
\mathrm{R} \\
\mathrm{R} \\
-\end{array}$ & $\begin{array}{l}\bar{z} \\
\bar{z} \\
\bar{z}\end{array}$ & $\begin{array}{l}\bar{z} \\
\overline{-} \\
\overline{-}\end{array}$ & $\begin{array}{l}\mathrm{F} \\
\mathrm{F} \\
\mathrm{R} \\
\mathrm{R} \\
\mathrm{R}\end{array}$ & $\frac{R}{R}$ & $\begin{array}{l}\bar{z} \\
\bar{z} \\
\overline{-}\end{array}$ & $\begin{array}{l}\overline{-} \\
\frac{\bar{R}}{\bar{F}}\end{array}$ & $\begin{array}{l}\overline{-} \\
\overline{-} \\
-\end{array}$ \\
\hline $19 \mathrm{H}-6,60-62$ & $\begin{array}{l}\mathrm{F} \\
\mathrm{C} \\
\mathrm{A}\end{array}$ & $\begin{array}{l}\mathrm{M} \\
\mathrm{P} \\
\mathrm{M}\end{array}$ & $\begin{array}{l}\overline{-} \\
\bar{I} \\
\overline{-}\end{array}$ & $\begin{array}{l}\bar{z} \\
\bar{z} \\
\overline{-}\end{array}$ & $\begin{array}{l}\bar{z} \\
\bar{z} \\
\bar{z}\end{array}$ & $\begin{array}{l}\bar{z} \\
\bar{z} \\
\bar{z}\end{array}$ & $\begin{array}{l}\overline{-} \\
\overline{-} \\
-\end{array}$ & $\begin{array}{l}\mathrm{F} \\
\mathrm{R}\end{array}$ & $\begin{array}{l}\overline{\mathrm{R}} \\
\overline{-} \\
-\end{array}$ & $\begin{array}{l}\mathrm{R} \\
\overline{\mathrm{R}} \\
\mathrm{R} \\
\mathrm{R}\end{array}$ & $\begin{array}{l}\bar{z} \\
\overline{-} \\
\overline{-}\end{array}$ & $\begin{array}{l}\bar{z} \\
\bar{z} \\
\bar{R}\end{array}$ & $\begin{array}{l}\bar{z} \\
\bar{z} \\
\overline{-}\end{array}$ & $\begin{array}{l}\overline{-} \\
\bar{z} \\
\bar{R}\end{array}$ & $\begin{array}{l}\mathrm{R} \\
\overline{\mathrm{R}} \\
\mathrm{R} \\
-\end{array}$ & $\begin{array}{l}\bar{z} \\
\bar{z} \\
\overline{-}\end{array}$ & $\begin{array}{l}\bar{z} \\
\bar{z} \\
\overline{-}\end{array}$ & $\begin{array}{l}\mathrm{R} \\
\mathrm{R} \\
-\end{array}$ & $\mathrm{R}$ & $\begin{array}{l}\overline{-} \\
\overline{-} \\
\bar{z}\end{array}$ & $\begin{array}{l}\mathrm{R} \\
\mathrm{R} \\
\mathrm{R} \\
\mathrm{R} \\
\mathrm{R}\end{array}$ & $\begin{array}{l}\frac{\mathrm{R}}{-} \\
\frac{-}{-}\end{array}$ \\
\hline $22 \mathrm{H}-3,60-62$ & $\begin{array}{l}\mathrm{F} \\
\mathrm{F}\end{array}$ & $\begin{array}{l}\mathrm{M} \\
\mathrm{M} \\
\mathrm{P} \\
\mathrm{P} \\
\mathrm{P}\end{array}$ & $\begin{array}{l}\bar{I} \\
\bar{I} \\
\bar{y}\end{array}$ & $\begin{array}{l}- \\
- \\
-\end{array}$ & $\begin{array}{l}\bar{z} \\
\bar{z} \\
\overline{-}\end{array}$ & $\begin{array}{l}\bar{z} \\
\overline{-}\end{array}$ & $\begin{array}{l}\overline{-} \\
\overline{-} \\
\overline{-}\end{array}$ & $\mathrm{R}$ & $\begin{array}{l}\overline{-} \\
\overline{-} \\
\bar{R}\end{array}$ & $\begin{array}{l}\mathrm{R} \\
\overline{\mathrm{R}} \\
\mathrm{R} \\
-\end{array}$ & $\begin{array}{l}\bar{z} \\
\bar{z} \\
\bar{z}\end{array}$ & $\begin{array}{l}\frac{F}{R} \\
- \\
-\end{array}$ & $\begin{array}{l}\bar{I} \\
\bar{I}\end{array}$ & $\begin{array}{l}\overline{-} \\
\bar{Z} \\
\bar{R} \\
\mathrm{R}\end{array}$ & $\begin{array}{l}\mathrm{R} \\
\overline{-} \\
\bar{R} \\
\mathrm{R}\end{array}$ & $\begin{array}{l}\overline{-} \\
\overline{-} \\
-\end{array}$ & $\begin{array}{l}\bar{z} \\
\overline{-} \\
\overline{-}\end{array}$ & $\begin{array}{l}\bar{z} \\
\overline{-} \\
\overline{-}\end{array}$ & $\begin{array}{l}\mathrm{R} \\
\frac{\mathrm{R}}{\mathrm{R}} \\
\end{array}$ & $\begin{array}{l}\overline{-} \\
\overline{-} \\
\overline{-}\end{array}$ & $\begin{array}{l}\mathrm{F} \\
\bar{R} \\
\mathrm{R} \\
\mathrm{R}\end{array}$ & $\begin{array}{l}\overline{-} \\
\overline{-} \\
-\end{array}$ \\
\hline $\begin{array}{l}24 \mathrm{H}-2,60-62 \\
24 \mathrm{H}-6,60-62\end{array}$ & $\begin{array}{l}\mathrm{C} \\
\mathrm{C}\end{array}$ & $\begin{array}{l}\mathrm{M} \\
\mathrm{M} \\
\mathrm{M} \\
\mathrm{M} \\
\mathrm{M}\end{array}$ & $\begin{array}{l}\overline{-} \\
\bar{I} \\
\overline{-}\end{array}$ & $\begin{array}{l}\overline{-} \\
\overline{-} \\
-\end{array}$ & $\begin{array}{l}\bar{z} \\
\bar{z} \\
\bar{z}\end{array}$ & $\begin{array}{l}\bar{z} \\
\bar{z} \\
\overline{-}\end{array}$ & $\begin{array}{l}\overline{-} \\
\overline{-} \\
\bar{R}\end{array}$ & $\begin{array}{l}\mathrm{R} \\
\mathrm{R}\end{array}$ & $\begin{array}{l}\frac{-}{\mathrm{R}} \\
\frac{\bar{R}}{\mathrm{R}}\end{array}$ & $\begin{array}{l}\bar{R} \\
\bar{R} \\
R\end{array}$ & $\begin{array}{l}\mathrm{R} \\
\mathrm{R}\end{array}$ & $\begin{array}{l}\mathrm{R} \\
\mathrm{R} \\
\mathrm{F} \\
\mathrm{R} \\
-\end{array}$ & 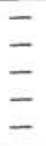 & $\frac{\frac{\mathrm{R}}{\mathrm{R}}}{-}$ & $\begin{array}{l}\overline{-} \\
\frac{\mathrm{R}}{\mathrm{R}}\end{array}$ & $\begin{array}{l}- \\
\overline{-} \\
-\end{array}$ & $\begin{array}{l}\bar{z} \\
\overline{-} \\
-\end{array}$ & $\begin{array}{l}\overline{-} \\
\overline{-} \\
-\end{array}$ & $\begin{array}{l}\bar{R} \\
R \\
R \\
R\end{array}$ & $\begin{array}{l}\overline{-} \\
\overline{-} \\
\overline{-}\end{array}$ & $\begin{array}{l}\mathrm{R} \\
\mathrm{R} \\
\mathrm{R} \\
\mathrm{R} \\
\mathrm{R}\end{array}$ & $\begin{array}{l}- \\
- \\
- \\
-\end{array}$ \\
\hline
\end{tabular}

Note: Abundance: $\mathrm{A}=$ abundant, $\mathrm{C}=$ common, $\mathrm{F}=$ few, $\mathrm{R}=$ rare; preservation: $\mathrm{G}=\operatorname{good}, \mathrm{M}=$ moderate, $\mathrm{P}=$ poor; lowercase letters indicate reworking. 
Table 9 (Continued).

\begin{tabular}{|c|c|c|c|c|c|c|c|c|c|c|c|c|c|c|c|c|c|c|c|c|c|}
\hline 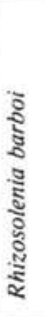 & 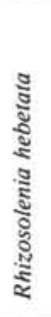 & 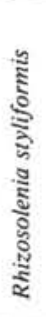 & 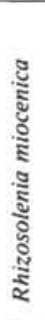 & 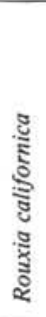 & 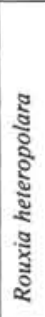 & 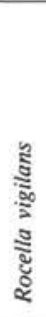 & 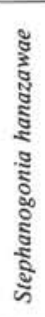 & 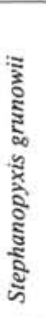 & 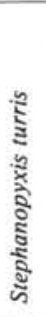 & 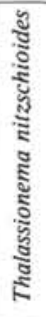 & 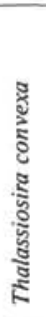 & 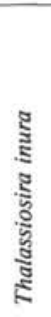 & 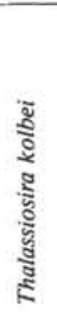 & 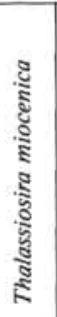 & 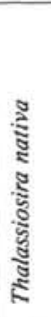 & 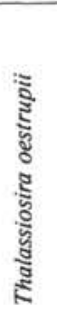 & 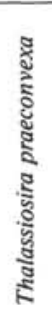 & 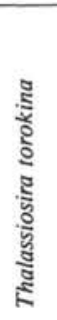 & 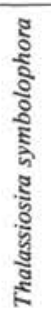 & 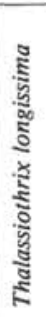 & 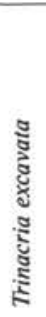 \\
\hline- & $\mathrm{R}$ & $R$ & - & - & - & - & - & - & - & $\mathbf{R}$ & - & $R$ & - & - & - & $\mathbf{R}$ & - & - & $R$ & $F$ & - \\
\hline- & $\mathrm{R}$ & $\mathrm{R}$ & - & - & - & - & - & - & $\mathbf{R}$ & $\mathrm{R}$ & - & $\mathrm{R}$ & - & - & - & $\mathrm{R}$ & $=$ & - & $\mathrm{R}$ & $\mathrm{F}$ & - \\
\hline- & $R$ & - & - & - & - & - & - & - & - & R & - & R & - & - & - & $\mathrm{R}$ & - & - & R & C & - \\
\hline- & - & - & - & - & - & - & - & - & - & $\mathrm{R}$ & - & - & - & - & - & $\ddot{R}$ & - & - & - & $F$ & - \\
\hline- & - & $R$ & - & - & - & - & - & - & - & $\mathbf{R}$ & - & - & - & - & - & R & - & - & - & $\mathrm{F}$ & - \\
\hline- & - & - & - & - & - & - & - & - & - & $\mathbf{R}$ & - & $\mathrm{R}$ & - & - & - & - & - & - & $\mathbf{R}$ & $\mathrm{F}$ & - \\
\hline- & - & $\mathrm{R}$ & - & - & - & - & - & - & - & $\mathrm{F}$ & - & $\ddot{R}$ & - & - & - & $\mathbf{R}$ & - & - & - & R & - \\
\hline- & R & $\mathrm{R}$ & - & - & - & - & - & - & - & $\mathrm{F}$ & - & $\hat{R}$ & - & - & - & R & - & - & - & C & - \\
\hline- & - & - & - & - & - & - & - & - & $\mathbf{R}$ & $\mathrm{F}$ & - & 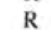 & - & - & - & $\mathbf{R}$ & - & - & - & $F$ & - \\
\hline $\mathbf{R}$ & - & $\mathbf{R}$ & - & - & - & - & - & - & - & $\mathrm{F}$ & - & - & - & - & - & $\mathbf{F}$ & - & - & - & $\mathbf{R}$ & - \\
\hline R & - & $\mathrm{R}$ & - & - & - & - & - & - & - & C & - & $\mathrm{R}$ & $\mathrm{R}$ & - & - & $\mathrm{R}$ & - & - & - & $\mathbf{R}$ & - \\
\hline $\mathrm{R}$ & - & $\mathrm{R}$ & - & - & - & - & - & - & - & $\mathrm{F}$ & - & $\mathrm{R}$ & $\mathrm{F}$ & - & - & R & - & - & - & $\mathbf{R}$ & - \\
\hline R & $\mathbf{R}$ & $\mathrm{R}$ & - & - & - & - & - & - & - & $\mathrm{R}$ & - & - & $\mathrm{R}$ & - & - & - & - & $\mathbf{R}$ & - & $\mathbf{R}$ & - \\
\hline R & - & $\mathrm{R}$ & - & - & R & - & - & - & $\mathrm{R}$ & R & - & $\mathrm{R}$ & $\hat{R}$ & - & - & $\mathbf{R}$ & - & $\mathrm{R}$ & - & R & - \\
\hline $\mathbf{R}$ & $\mathbf{R}$ & $\mathbf{R}$ & - & - & - & - & - & - & $\mathrm{R}$ & R & - & R & $\mathbf{R}$ & - & - & $\mathbf{R}$ & - & - & $\mathbf{R}$ & $\mathbf{R}$ & - \\
\hline $\mathrm{R}$ & $\mathrm{R}$ & $R$ & - & R & - & - & - & - & $R$ & $F$ & - & - & $R$ & - & - & - & - & - & - & R & - \\
\hline- & - & - & - & $\mathbf{R}$ & - & - & - & - & $\mathbf{R}$ & C & - & $\mathbf{R}$ & $\mathrm{R}$ & - & - & - & - & - & - & R & - \\
\hline- & $\mathbf{R}$ & - & - & - & - & - & - & - & R & C & - & $\ddot{R}$ & $\hat{R}$ & - & - & $\mathbf{R}$ & - & - & $\mathbf{R}$ & $\mathrm{R}$ & - \\
\hline- & - & - & - & $R$ & - & - & - & $\mathrm{F}$ & C & $\mathrm{F}$ & - & $\vec{F}$ & $\mathrm{R}$ & - & - & $\mathbf{R}$ & - & - & - & F & - \\
\hline $\mathrm{R}$ & $\mathrm{R}$ & $\mathrm{R}$ & - & - & - & - & - & F & $\mathrm{F}$ & F & - & $\mathrm{F}$ & $\mathrm{R}$ & - & - & $\ddot{R}$ & - & - & $\mathrm{R}$ & $\mathrm{F}$ & - \\
\hline$R$ & - & $\mathrm{R}$ & - & - & $R$ & - & - & - & $\mathrm{R}$ & $F$ & - & $R$ & - & - & - & $\mathbf{R}$ & - & $\mathrm{R}$ & $R$ & $F$ & - \\
\hline- & $=$ & $\hat{R}$ & - & - & - & - & - & - & $\hat{R}$ & $\mathrm{~F}$ & - & $\hat{R}$ & - & - & - & $\widehat{R}$ & - & $\mathrm{R}$ & $\mathrm{R}$ & $\therefore$ & - \\
\hline $\mathrm{R}$ & - & $\mathrm{R}$ & - & - & $R$ & - & - & - & $\mathrm{R}$ & F & - & $\mathrm{F}$ & - & - & - & $\mathbf{R}$ & - & - & - & R & - \\
\hline- & - & $\mathrm{R}$ & - & - & - & - & - & - & $\mathrm{R}$ & $\mathrm{F}$ & - & $\mathrm{R}$ & - & - & - & $\mathbf{R}$ & - & $\mathbf{R}$ & $\mathbf{R}$ & $F$ & - \\
\hline $\mathrm{R}$ & - & - & - & - & - & - & - & - & $\mathrm{F}$ & F & - & F & - & - & - & $\mathbf{R}$ & - & - & $\mathbf{R}$ & $\mathrm{F}$ & - \\
\hline- & - & - & - & - & - & - & - & - & - & $R$ & - & $R$ & - & - & - & $R$ & - & - & $=$ & $R$ & - \\
\hline- & - & $\mathrm{R}$ & - & $\mathbf{R}$ & - & - & - & - & $\mathrm{R}$ & $\ddot{R}$ & & R & & - & & $\mathrm{R}$ & - & - & - & & - \\
\hline$R$ & - & - & - & $R$ & - & - & - & - & $\vec{F}$ & R & - & $\mathrm{F}$ & - & - & - & $\mathbf{R}$ & - & R & R & R & - \\
\hline $\mathrm{R}$ & $\mathrm{R}$ & $\mathrm{R}$ & - & - & R & - & - & - & R & $\mathrm{R}$ & - & $\mathrm{R}$ & - & - & 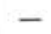 & $\mathrm{R}$ & - & - & $\mathbf{R}$ & $F$ & - \\
\hline $\mathbf{R}$ & - & - & - & F & - & - & - & - & $R$ & $\mathrm{R}$ & - & R & - & - & - & $\mathrm{R}$ & - & - & $\mathbf{R}$ & $\mathrm{F}$ & - \\
\hline$R$ & - & $\mathrm{R}$ & - & - & $F$ & - & - & - & $R$ & $F$ & - & $\bar{F}$ & - & - & - & $\mathrm{R}$ & - & - & - & $F$ & - \\
\hline$R$ & $\mathrm{R}$ & - & - & $R$ & - & - & - & - & $\mathrm{F}$ & $\mathrm{R}$ & & $\mathrm{F}$ & - & - & 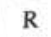 & $\mathbf{R}$ & - & - & $\mathbf{R}$ & A & - \\
\hline$\vec{F}$ & - & $\mathrm{R}$ & R & - & - & - & $\mathbf{R}$ & - & $\mathrm{F}$ & R & - & $\mathrm{R}$ & - & - & $\mathrm{R}$ & $\mathrm{R}$ & - & $\mathrm{R}$ & $\mathbf{R}$ & C & - \\
\hline$R$ & - & $\mathrm{R}$ & - & $R$ & - & - & - & - & $F$ & $\mathrm{~F}$ & - & $\hat{R}$ & - & - & R & $R$ & - & $R$ & - & A & - \\
\hline $\mathrm{R}$ & - & $\mathrm{R}$ & - & $\mathrm{R}$ & - & - & - & - & $\mathrm{R}$ & C & - & $\mathrm{F}$ & - & - & - & $\mathrm{R}$ & - & R & - & F & - \\
\hline- & - & $\mathrm{R}$ & - & $\mathbf{R}$ & - & - & - & - & $\mathrm{F}$ & $\mathbf{R}$ & - & $\mathrm{F}$ & - & - & - & $\mathrm{R}$ & - & - & - & $\mathrm{A}$ & - \\
\hline- & - & $\ddot{R}$ & - & $\ddot{R}$ & - & - & - & - & $\mathrm{R}$ & $\hat{F}$ & - & $\mathrm{R}$ & & $=$ & & R & 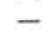 & $\mathrm{R}$ & - & & - \\
\hline $\mathbf{R}$ & - & $\mathbf{R}$ & - & - & - & - & - & - & $\mathrm{R}$ & $\mathrm{R}$ & 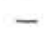 & $\mathrm{R}$ & - & - & $\mathrm{R}$ & $\mathrm{R}$ & - & - & $\mathbf{R}$ & C & - \\
\hline $\mathrm{R}$ & - & $\ddot{R}$ & - & $\mathrm{R}$ & R & - & $\mathrm{R}$ & - & - & $\mathrm{F}$ & - & $\hat{R}$ & - & - & - & R & - & $\mathbf{R}$ & R & $F$ & - \\
\hline $\mathrm{R}$ & $\mathrm{R}$ & $\mathrm{R}$ & - & $\mathrm{R}$ & R & - & - & - & $\mathrm{R}$ & $\mathrm{F}$ & - & $\hat{R}$ & - & - & $\mathbf{R}$ & $\mathrm{R}$ & - & $R$ & - & A & - \\
\hline $\mathbf{R}$ & $\mathrm{R}$ & $\mathrm{R}$ & - & - & R & - & $\mathbf{R}$ & - & $\mathrm{R}$ & $\mathrm{F}$ & - & $\mathbf{R}$ & - & - & - & - & - & $\mathrm{R}$ & $\mathbf{R}$ & $\mathrm{C}$ & - \\
\hline $\mathrm{R}$ & - & - & - & - & $R$ & . & - & 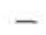 & $\cdots$ & F & & - & 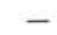 & - & - & - & - & R & - & C & - \\
\hline $\mathrm{R}$ & $\mathbf{R}$ & $\mathbf{R}$ & - & - & $\mathrm{R}$ & - & - & - & - & C & - & - & - & - & R & $\mathbf{R}$ & - & $\mathbf{R}$ & - & $F$ & - \\
\hline $\mathbf{R}$ & $\mathrm{R}$ & $\mathrm{R}$ & - & $\mathbf{R}$ & $\mathrm{R}$ & $R$ & - & - & - & $\mathrm{F}$ & - & - & - & - & - & - & - & $\mathrm{R}$ & - & $\mathrm{F}$ & - \\
\hline $\mathrm{R}$ & - & $\mathrm{R}$ & R & $\mathrm{R}$ & R & R & - & - & $\mathrm{R}$ & $\mathrm{F}$ & - & - & - & - & - & - & - & R & - & $\mathrm{F}$ & $\mathrm{R}$ \\
\hline$F$ & $\mathbf{R}$ & $\mathrm{R}$ & - & $\mathbf{R}$ & $\mathrm{R}$ & - & - & - & - & $\mathrm{F}$ & $\mathrm{R}$ & - & - & - & - & - & - & $F$ & - & $F$ & $\mathrm{R}$ \\
\hline $\mathbf{R}$ & - & $\mathrm{R}$ & - & - & $R$ & - & - & - & - & $\mathrm{F}$ & - & - & - & - & - & - & - & $\mathbf{R}$ & - & 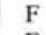 & $\mathbf{R}$ \\
\hline $\mathrm{R}$ & $\mathbf{R}$ & $\mathbf{R}$ & - & - & $\mathrm{F}$ & - & - & - & - & $\mathrm{F}$ & - & - & - & $\mathbf{R}$ & $\mathrm{R}$ & - & - & $R$ & R & $F$ & - \\
\hline$\ddot{R}$ & $\ddot{R}$ & - & - & - & $\mathrm{R}$ & - & - & - & - & $\mathrm{F}$ & - & - & - & - & $\ddot{R}$ & - & $\mathbf{R}$ & $\mathrm{R}$ & $\mathrm{R}$ & $\mathrm{F}$ & - \\
\hline R & - & $\mathbf{R}$ & - & - & R & - & - & - & - & $\mathrm{R}$ & - & - & - & - & - & - & - & F & R & R & R \\
\hline
\end{tabular}

\section{Prydz Bay}

\section{Site 739}

Of the five Leg 119 sites occupied in Prydz Bay (Table 1 and Fig. 1), only the samples examined from Site 739 contained a diatom flora useful for stratigraphic control. Site 739 , positioned on the outer continental shelf, penetrated a thick sequence ( 486.8 $\mathrm{m}$ ) of glacial sediments. Diatoms were recovered from random samples and provide the primary means of stratigraphic control for the sequence recovered. Figure 8 shows the stratigraphic log, core recovery, and the samples examined from this interval, as well as the assigned ages of the samples containing diatoms. Of 
Hole745B

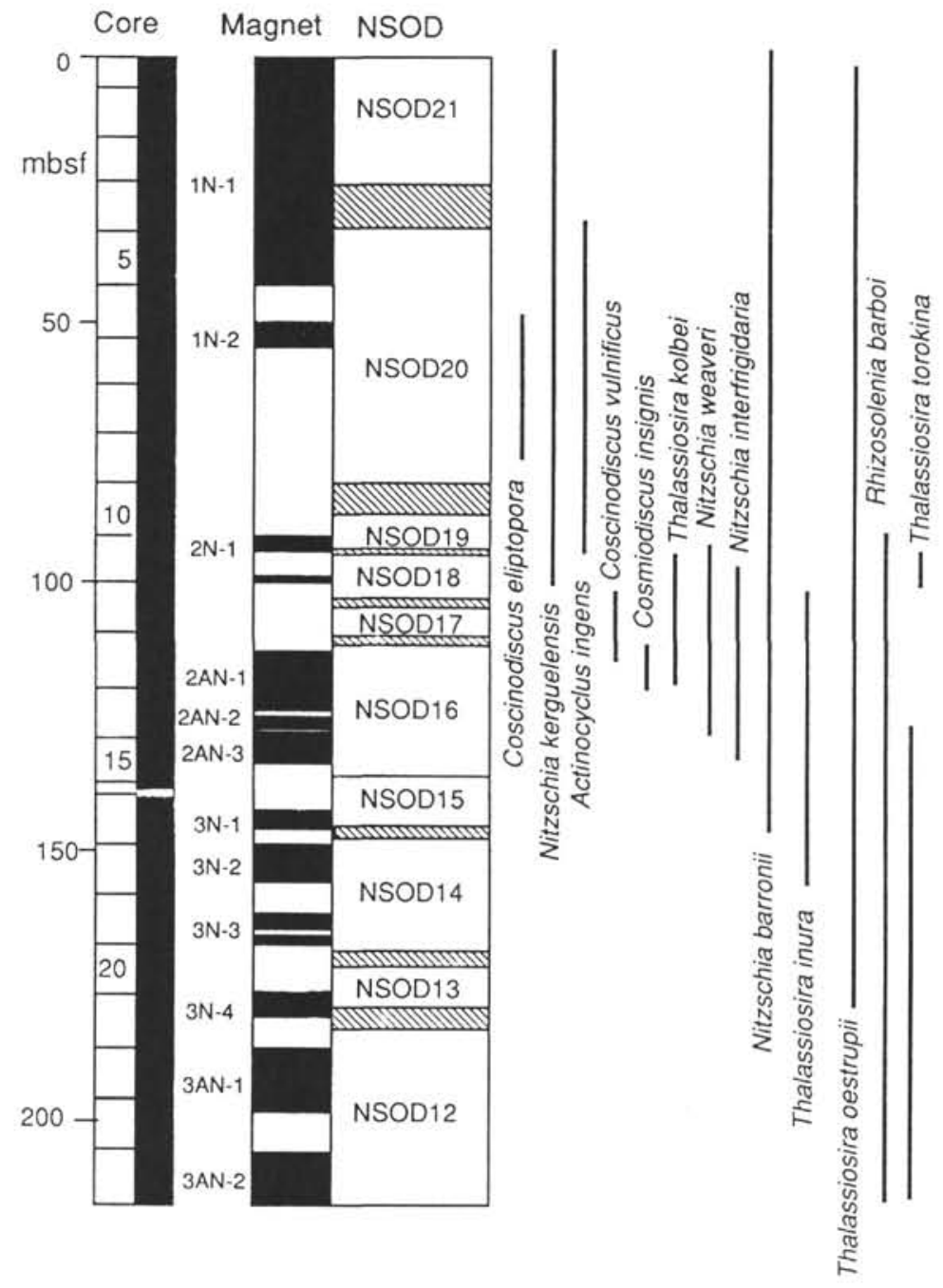

Figure 6. Stratigraphic recovery (Barron, Larsen, et al., 1989), paleomagnetics (H. Sakai, pers. comm., 1989), assigned zones, and the range of selected species from Hole 745B. The width of a range bar indicates relative abundance (see tables for specifics).

the 60 samples examined 35 contained diatoms. The abundance and preservation of diatoms in these samples varies, but in general, diatoms are rare and poorly preserved (Table 11). Four distinct chronostratigraphic intervals (Quaternary, ?Pliocene, late Miocene, and early Oligocene) are recognized based on diatom biostratigraphy.

A Quaternary age is assigned to the upper two cores based on a moderately to well-preserved diatom assemblage consisting of Nitzschia kerguelensis, Thalassiosira inura, Actinocyclus actinochilis, Nitzschia barronii, Thalassiosira lentiginosa, and Nitzschia curta. Although assignment is tentative because of possible reworking, the absence of both Actinocyclus ingens and Rhizosolenia barboi combined with the presence of $N$. curta and $T$. lentiginosa suggests placement of this interval into NSOD Zone 21 . The occurrence of $N$. kerguelensis in this interval indicates that this sequence has an age younger than the first occurrence of N. kerguelensis at $2.0 \mathrm{Ma}$ (in the Kerguelen Plateau region).

Samples containing diatoms from Cores 119-739C-8R, 119739C-13R, 119-739C-14R, 119-739C-18R, and 119-739C-19R are assigned a Pliocene age based on the diatoms observed from these intervals. A ?Pliocene age is suggested for Sample 119739C-8R-CC based on the rare occurrence of Nitzschia praeinterfrigidaria (last occurrence in the Kerguelen Plateau region is at 3.6 Ma). However, the rare specimens may be reworked, which is suggested by the lack of other diatoms, including other Pliocene species.

The interval of Cores $119-739 \mathrm{C}-13 \mathrm{H}$ and $119-739 \mathrm{C}-14 \mathrm{H}$ is assigned an early Pliocene age based on the occurrence of Nitzschia praecurta, Nitzschia cylindrus, Rouxia peragalli, Thalassiosira lentiginosa, and Nitzschia praeinterfrigidaria. The occurrence of few specimens of $N$. praeinterfrigidaria suggests an age for this sequence younger than the first occurrence of $N$. praeinterfrigidaria at $4.5 \mathrm{Ma}$ (Table 2). Although reworking of glacially compacted sediments cannot be completely ruled out, the absence in this interval of the diatom assemblage observed in Cores 119-739C-1H and 119-739C-2H (i.e., N. kerguelensis, Nitzschia ritscheri, and Thalassiosira gravida) suggests that these samples are stratigraphically below the first occurrence of these 
species and have an early Pliocene age. The occurrence of $N$. cf. praeinterfrigidaria, $N$. barronii, and $T$. inura also suggests a Pliocene age for the samples examined from Cores $119-739 \mathrm{C}-18 \mathrm{H}$ and $119-739 \mathrm{C}-19 \mathrm{H}$.

Samples examined from Cores 119-739C-20R through 119$739 \mathrm{C}-22 \mathrm{R}$ are placed in the late Miocene NSOD Zone 12 based on the occurrence of few Denticulopsis hustedtii, Nitzschia cylindrica, Thalassiosira burckliana, and Rouxia californica. No samples were available from Core 119-739C-24R. The presence of Nitzschia cylindrica and Thalassiosira burckliana suggests an age of 7.4 to $6.2 \mathrm{Ma}$ according to the ranges of these taxa at Site 746 (Fig. 7).

Samples examined from Cores 119-739C-25R through 119$739 \mathrm{C}-38 \mathrm{R}$ are assigned an earliest Oligocene age. Examined samples from stratigraphically below Core 119-739C-38R are barren of diatoms. The species observed from Cores 119-739C$25 \mathrm{R}$ through 119-739C-38R (Table 11) are indicative of the Oligocene. Species characteristic of this interval include Hemiaulus caracteristicus, Stephanopyxis grunowii, Melosira architecturalis, Goniothecium odontella, Pyxilla reticulata, Asteromphalus oligocenicus, Kiesielviella carina, and Pseudostictodiscus picus. The occurrence of these species without Rocella gelida, $R$. vigilans, or Synedra jouseana (characteristic of the late Oligocene) suggests an early Oligocene age for this interval.

Shore-based ${ }^{87} \mathrm{Sr} /{ }^{86} \mathrm{Sr}$ analysis of selected bivalve and scaphopod fragments from Cores 119-739C-25R, 119-739C-29R, 119-739C-31R, 119-739C-33R, 119-739C-34R, and 119-739C36R suggests that samples from Cores 119-739C-31R through 119-739C-36R record nearly continuous deposition from 23.4 to $29.5 \mathrm{Ma}$ (Thierstein et al., this volume). The analytical results from Cores $119-739 \mathrm{C}-25 \mathrm{R}$ through $119-739 \mathrm{C}-29 \mathrm{R}$ suggest that samples were obtained from a disturbed sequence. The calculated ages of these cores range from 26 through $28.9 \mathrm{Ma}$, but are not positioned in stratigraphic order (Thierstein et al., this volume).

The preceding analytical results, if correct, argue that the Oligocene diatom assemblage was reworked into unfossiliferous sediment of younger age. However, the strontium values are considered tentative and questionable on the basis of (1) inconsistent stratigraphic trend between the lower and upper stratigraphic samples, (2) lack of SEM studies to evaluate the mineral status of the aragonite, and 3) extremely low sedimentation rates ( $7.5 \mathrm{~m} / \mathrm{m} . \mathrm{y}$.) for glacial-derived sediments when using $\mathrm{Sr}$ values for age control (see Barron et al., this volume, for further discussion).

Although the possibility that the assemblage is reworked can not be completely ruled out, the following points support an early Oligocene age assignment.

1. Table 11 shows the occurrence of selected diatoms from Hole 739 C. As previously discussed, the diatom assemblages observed from this interval indicate a biostratigraphic sequence positioned in stratigraphic order, from Quaternary (Cores 119739C-1R and 119-739C-2R) through ?Pliocene (Cores 119739C-8R, 119-739C-13R, 119-739C-14R, 119-739C-18R, and 119-739C-19R), late Miocene (Cores 119-739C-20R and 119739C-22R), and Oligocene (Cores 119-739C-25R through 119$739 \mathrm{C}-38 \mathrm{R})$. The assemblages observed from these chronostratigraphic intervals are comparable to those observed from the Kerguelen Plateau region (see the preceding). It should be pointed out that the reworking of specific diatom frustules, such as Denticulopsis hustedtii in Samples 119-739C-13R-CC, 119739C-14R-CC, and 119-739C-17R-CC, did occur. In addition, downhole contamination is also evident (occurrence of N. kerguelensis in Samples 119-739C-25R-1, $68 \mathrm{~cm}$, and 119-739C$29 \mathrm{R}-4,58 \mathrm{~cm}$ ).

2. The diatom assemblage observed from Cores $119-739 \mathrm{C}$ $25 \mathrm{R}$ through $119-739 \mathrm{C}-38 \mathrm{R}$ is similar in composition to that described by Harwood (in press) from the CIROS-1 drill core, western Ross Sea, Antarctica. Diatoms recovered from the CIROS-1 site are indicative of the lower Oligocene Rhizosolenia gravida Zone of Fenner (1985; equal to the lower portion of the $R$. oligocenica Zone as used in this manuscript) and the upper Oligocene-lower Miocene Rocella vigilans Zone of Fenner (1985). Harwood et al. (in press) further reported that based on diatom stratigraphy the interval representing the lower upper Oligocene (equivalent to the $R$. antarctica Zone of Fenner, 1985) through the lower upper Oligocene (lower $R$. vigilans Zone) is missing from the CIROS-1 drill site. The base of this disconformity is constrained by the last occurrences of the Pyxilla group, $H$. caracteristicus, Stephanopyxis superba, Stephanopyxis splendidus (referred to in this manuscript as Thalassiosira hydra), and Sceptroneis lingulatus. Directly above this disconformity is positioned the first occurrence of Synedra jouseana, and further upsection is placed the first occurrence of Lisitzinia ornata.

At Site 739 a similar succession in the diatom assemblage is observed for the interval assigned to the lower Oligocene. Of the species noted at CIROS-1, H. caracteristicus (Sections 119739C-26R-CC, 119-739C-30R-1, 119-739C-32R-CC, and 119739C-33R-4 and Cores 119-739C-35R through 119-739C-37R), S. superba (Sections 119-739C-26R-CC, 119-739C-30R-1, and 119-739C-37R-1), T. hydra (Section 119-739C-26R-CC), and the Pyxilla group (in the majority of samples) are observed, which suggests a comparable age. This lower Oligocene sequence is unconformably overlain by diamictite of late Miocene age. The disconformity is constrained to Core 119-739C-24R and removes upper Oligocene through lower upper Miocene sediments.

\section{DISCUSSION}

Drilling results from Leg 119 provide an opportunity to calibrate biostratigraphic events to the magnetostratigraphy gleaned from Sites 744 through 746 . Table 12 summarizes the chronostratigraphic placement of specific diatom events from these sites based on the sample constraints for individual biostratigraphic events and the calibration of these events to the magnetostratigraphy (H. Sakai, pers. comm., 1989). Combining the results from each site into a composite sequence indicates that a nearly continuous sequence of biostratigraphic and magnetostratigraphic events was recovered from the Kerguelen Plateau region representing the Quaternary through early Oligocene. These events provide a chronostratigraphic tie-point for the Indian Ocean sector of the Southern Ocean. Comparison of the estimated ages of each event to previously determined ages elsewhere in the Southern Ocean (i.e., Gersonde and Burckle, 1990) allows development of a regional Southern Ocean stratigraphy, as previously discussed in this manuscript.

Further evidence as to the stratigraphic usefulness of the zonation proposed here is presented in Table 13, which shows the stratigraphic constraints of diatom events for samples reexamined from DSDP Sites 266 and 278 . The composite stratigraphic sequences recovered from these two sites provide a nearly continuous record for the Miocene. However, the latest Miocene is missing from both sites and is represented by a hiatus with an approximate duration of 5.1 m.y. (4.8-9.9 Ma) at Site 266.

\section{CONCLUSIONS}

Diatoms examined from the 11 sites occupied during Leg 119 provide a regional stratigraphic framework for the Indian Ocean sector of the Southern Ocean and when correlated to other stratigraphic sequences previously recovered from elsewhere in the Indian Ocean provide a stratigraphic framework for the Southern Ocean overall. The resulting diatom biostratigraphy integrates previously established zonations with new diatom zones proposed here for previously problematic stratigraphic intervals. 
Table 10. Occurrence of selected diatom species observed from Hole 746A.

\begin{tabular}{|c|c|c|c|c|c|c|c|c|c|c|c|c|c|c|c|c|c|c|c|c|c|c|}
\hline $\begin{array}{l}\text { Core, section, } \\
\text { interval }(\mathrm{cm})\end{array}$ & 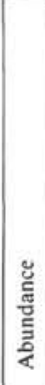 & 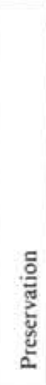 & 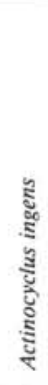 & 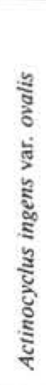 & 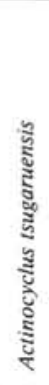 & 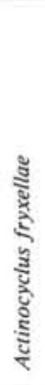 & 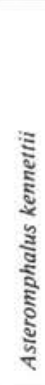 & 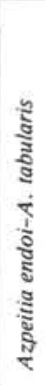 & 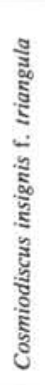 & 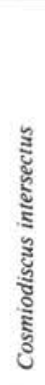 & 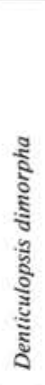 & 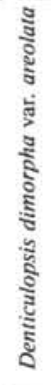 & 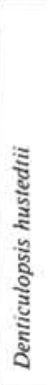 & 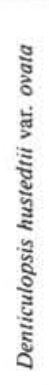 & 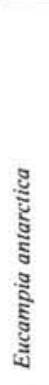 & 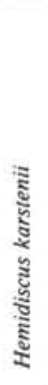 & 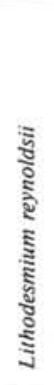 & 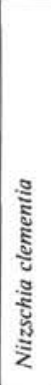 & 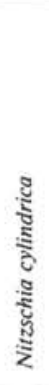 & 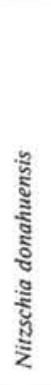 & 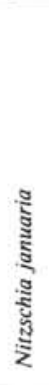 & 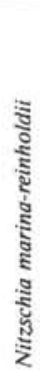 \\
\hline \multicolumn{23}{|l|}{$19-746 \mathrm{~A}$} \\
\hline $4 \mathrm{H}-1,60$ & A & M & - & - & - & - & - & - & - & $\mathbf{R}$ & - & - & F & - & & - & - & & & - & & R \\
\hline & A & $\mathrm{M}$ & - & - & - & - & - & $\mathbf{R}$ & - & - & - & - & $\mathrm{F}$ & - & - & - & - & - & - & - & - & - \\
\hline $4 \mathrm{H}-3,60$ & A & G & - & - & - & - & - & - & - & $\mathrm{F}$ & - & - & $\mathrm{F}$ & - & $R$ & - & - & - & - & - & - & R \\
\hline $4 \mathrm{H}-4,60$ & A & G & - & - & - & - & - & - & - & $\mathrm{F}$ & - & - & $\mathrm{F}$ & - & - & - & - & - & - & - & - & - \\
\hline $4 \mathrm{H}-5,60$ & A & $\mathrm{M}$ & - & $\mathrm{R}$ & - & - & - & - & $\mathbf{R}$ & $\mathrm{F}$ & $\mathrm{R}$ & - & $\mathrm{F}$ & - & - & - & - & - & $\mathrm{R}$ & - & - & - \\
\hline $4 \mathrm{H}-6,60$ & A & M & - & - & - & - & - & - & - & $\mathrm{F}$ & $\mathrm{R}$ & - & $\mathrm{F}$ & - & $\mathrm{R}$ & - & - & - & $\mathbf{R}$ & - & - & $\mathrm{R}$ \\
\hline & f & $\mathrm{M}$ & - & - & - & $\mathrm{R}$ & - & - & $\bar{c}$ & $\mathrm{~F}$ & & - & $\mathrm{F}$ & - & & - & - & 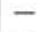 & - & - & - & - \\
\hline $5 \mathrm{H}-1,60$ & A & $\mathrm{M}$ & - & $\overline{\mathrm{p}}$ & - & $\overline{0}$ & - & R & $\mathrm{F}$ & $\mathrm{F}$ & p & - & $\mathrm{F}$ & - & $\mathbf{R}$ & - & - & - & $\mathrm{F}$ & R & - & $\mathrm{F}$ \\
\hline $5 \mathrm{H}-2,60$ & A & G & - & $\mathbf{R}$ & - & $\mathrm{R}$ & - & $\mathrm{R}$ & $\mathrm{F}$ & $\mathrm{F}$ & $\mathrm{R}$ & - & $\mathrm{F}$ & - & - & - & - & - & $\mathrm{F}$ & $\mathrm{R}$ & - & - \\
\hline $5 \mathrm{H}-3,60$ & A & $\mathrm{G}$ & - & - & - & $\mathrm{R}$ & - & $\mathrm{F}$ & $\mathrm{R}$ & $\mathrm{F}$ & k & - & $\mathrm{F}$ & - & $\mathrm{R}$ & & - & - & $\mathrm{R}$ & - & - & $\mathrm{F}$ \\
\hline $5 \mathrm{H}-4,60$ & C & M & - & - & - & $\mathrm{R}$ & - & - & - & $\mathrm{R}$ & $\mathbf{R}$ & - & C & - & - & - & - & - & - & - & - & - \\
\hline & & $\Lambda$ & & 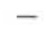 & - & & & p & - & $\bar{r}$ & & - & $\mathrm{F}$ & - & & & - & - & $\mathrm{F}$ & - & - & $\mathbf{R}$ \\
\hline SH-6, 60 & , & $N$ & 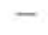 & - & - & $\mathrm{R}$ & - & $\mathrm{k}$ & - & $\mathrm{F}$ & $\mathrm{k}$ & - & $\mathrm{F}$ & - & & & - & - & $\mathrm{F}$ & - & - & - \\
\hline $5 \mathrm{H}-\mathrm{CC}$ & A & $\mathrm{M}$ & - & $\overline{0}$ & $\mathrm{R}$ & $\mathbf{R}$ & - & - & - & $\mathrm{F}$ & - & - & C & - & $R$ & - & - & - & - & $\mathrm{R}$ & - & $\mathrm{R}$ \\
\hline $6 \mathrm{H}-1,65$ & A & M & - & $\mathrm{R}$ & $\mathrm{R}$ & $\mathrm{F}$ & - & - & - & $\mathrm{R}$ & - & - & C & - & $\mathrm{R}$ & - & - & - & - & R & - & - \\
\hline $6 \mathrm{H}-2,65$ & A & G & - & $R$ & $F$ & $F$ & - & - & - & $\mathrm{F}$ & $\mathrm{R}$ & - & A & - & $\mathrm{R}$ & - & - & $\overline{8}$ & $R$ & $\mathbf{R}$ & - & - \\
\hline 6 & & c & - & 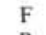 & $F$ & $\mathbf{R}$ & & & - & $\mathrm{F}$ & & - & & - & & & - & $\mathbf{R}$ & - & & & - \\
\hline 6 & & G & - & $\mathrm{R}$ & R & R & 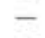 & - & - & P & - & - & & - & - & & - & - & - & $\mathbf{R}$ & - & - \\
\hline $6 \mathrm{H}-5,65$ & A & M & - & F & $R$ & R & - & - & - & $F$ & - & - & 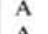 & - & - & 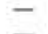 & - & - & $\mathrm{R}$ & $\bar{p}$ & - & $\mathrm{R}$ \\
\hline $6 \mathrm{H}-6,68$ & A & G & - & $\mathrm{K}$ & $\mathrm{R}$ & $\mathrm{R}$ & - & - & - & $\mathrm{R}$ & - & - & $\mathrm{A}$ & - & - & - & - & - & - & $\mathrm{R}$ & - & - \\
\hline 6 & A & M & - & $\mathrm{F}$ & - & $\mathrm{R}$ & - & - & - & $\mathrm{F}$ & - & - & $\mathrm{F}$ & - & $\mathrm{R}$ & - & - & - & - & - & - & - \\
\hline & & 0 & 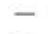 & $\mathrm{F}$ & - & $\mathrm{R}$ & & 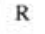 & - & $\mathrm{F}$ & - & - & $\because$ & - & & & - & 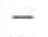 & $\mathrm{R}$ & & - & $\mathrm{R}$ \\
\hline 1-2, 60 & & $\mathrm{~N}$ & - & $\mathrm{R}$ & $R$ & $\mathrm{~F}$ & - & - & - & $\mathrm{R}$ & $\mathbf{R}$ & - & A & - & 1 & & - & - & $\mathbf{R}$ & - & - & $\mathrm{R}$ \\
\hline & & $\mathrm{G}$ & - & $\bar{r}$ & $R$ & R & - & - & - & $\mathrm{R}$ & - & - & A & - & - & & - & 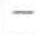 & - & R & - & $\overline{\mathrm{D}}$ \\
\hline 7H-4, 60 & A & $\mathrm{M}$ & - & $\mathrm{F}$ & $\mathrm{R}$ & $\mathbf{R}$ & - & - & - & $\mathrm{F}$ & - & - & A & - & - & - & - & - & $\mathrm{R}$ & $\mathbf{R}$ & - & $\mathbf{R}$ \\
\hline 7H-5, 60 & a & $\mathrm{M}$ & - & $\mathrm{F}$ & - & $\mathrm{R}$ & - & - & - & $\mathrm{F}$ & - & - & $A$ & - & R & - & - & - & - & $\mathrm{R}$ & - & $\mathrm{R}$ \\
\hline . & & c & 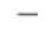 & $\mathrm{F}$ & R & $\mathrm{R}$ & & - & - & $\mathrm{R}$ & - & - & $A_{1}$ & - & 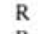 & & - & - & $\mathbf{R}$ & . & 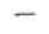 & - \\
\hline $\mathrm{CC}$ & & $\mathrm{N}$ & - & $=$ & $\mathbf{R}$ & $\mathbf{R}$ & - & - & - & $\mathrm{R}$ & - & - & C & - & $\mathrm{R}$ & - & - & - & - & 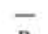 & - & - \\
\hline$-1,60$ & & G & - & 5 & $\mathrm{I}$ & - & - & $R$ & - & $P$ & - & - & A & - & $\mathrm{F}$ & $P$ & - & - & $\mathrm{R}$ & 0 & - & $\mathbf{R}$ \\
\hline $8 \mathrm{H}-2,60$ & A & $\mathrm{M}$ & - & $\mathrm{R}$ & $\mathbf{R}$ & - & - & - & - & $\mathrm{F}$ & - & - & A & - & $\mathrm{F}$ & $R$ & - & - & - & $\mathrm{R}$ & - & - \\
\hline $8 \mathrm{H}-3,60$ & A & N & - & - & $R$ & $\mathbf{R}$ & - & $\mathrm{F}$ & - & $F$ & - & - & A & - & F & $R$ & - & - & $\mathbf{R}$ & - & - & - \\
\hline & & N & - & & F & $\mathrm{F}$ & & & 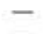 & D & - & - & & 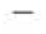 & & & 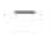 & 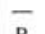 & & & - & - \\
\hline & & N & - & & $\mathbf{R}$ & - & - & 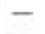 & - & $\mathrm{R}$ & - & - & A & - & $R$ & & - & $\mathrm{R}$ & - & & - & - \\
\hline & & $\mathrm{N}$ & - & & $\mathrm{F}$ & - & - & 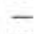 & - & $\mathbf{R}$ & - & - & A & - & $\mathrm{R}$ & & - & - & - & & - & - \\
\hline $9 \mathrm{H}-5,45$ & c & $\mathrm{M}$ & $R$ & - & $\mathrm{F}$ & $\mathrm{R}$ & - & - & - & $\mathrm{R}$ & $\mathrm{K}$ & - & A & - & - & - & - & - & - & - & - & - \\
\hline $9 \mathrm{H}-6,60$ & A & $N$ & $\mathrm{R}$ & - & $\mathrm{R}$ & $F$ & - & R & - & $\mathrm{R}$ & - & - & A & - & $\mathrm{F}$ & - & - & - & - & - & - & - \\
\hline & & $\mathrm{N}$ & - & & $\mathrm{F}$ & & - & & & & & 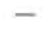 & & & & & - & & & & & - \\
\hline 60 & & $\mathrm{~N}$ & - & & $\mathrm{F}$ & $\mathrm{F}$ & - & 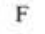 & - & & - & 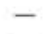 & A & - & $\mathrm{R}$ & - & $\mathrm{R}$ & - & - & & & - \\
\hline & & N & - & & $\mathrm{F}$ & $\mathbf{R}$ & 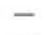 & - & - & 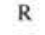 & - & - & A & - & - & $\mathbf{R}$ & $\mathrm{R}$ & - & - & & - & - \\
\hline $10 \mathrm{H}-3,60$ & A & $\mathrm{M}$ & - & - & $\mathrm{R}$ & - & - & $\mathbf{R}$ & - & - & - & - & A & - & - & - & - & - & - & - & - & - \\
\hline $10 \mathrm{H}-4,60$ & A $>$ & $\mathrm{N}$ & $\mathrm{F}$ & - & $\mathrm{F}$ & $\mathrm{R}$ & - & $\mathbf{R}$ & - & - & - & - & A & - & $R$ & - & - & - & - & - & - & - \\
\hline & & N & & & s & - & & & & & & & & & $F$ & & & & & & & - \\
\hline & & $\mathrm{N}$ & - & . & F & - & 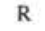 & & & & 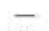 & & & $\pi$ & $\mathrm{F}$ & & 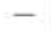 & & - & & - & - \\
\hline & & N & $\mathrm{R}$ & - & $\mathbf{R}$ & $\overline{0}$ & - & $\mathrm{R}$ & - & - & $\mathrm{R}$ & 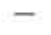 & 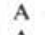 & $\mathrm{R}$ & $\overline{0}$ & 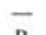 & - & - & - & - & - & - \\
\hline $11 \mathrm{H}-2,60$ & C & $\mathrm{M}$ & - & - & - & $\mathrm{R}$ & - & $\mathrm{F}$ & - & - & $\mathrm{F}$ & - & A & $\mathrm{R}$ & $\mathbf{R}$ & $\mathbf{R}$ & - & - & - & - & $\mathbf{R}$ & - \\
\hline 6 & & i & $\overline{0}$ & 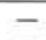 & $R$ & - & - & P & - & 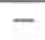 & $R$ & - & A & -1 & - & R & - & $\pi$ & - & - & & - \\
\hline & & & $\mathrm{R}$ & & $\pi$ & $\mathbf{R}$ & & & & & & - & & & $\mathrm{R}$ & & & & 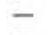 & & & - \\
\hline & & $\mathrm{N}$ & 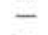 & & $\mathrm{F}$ & $\mathbf{R}$ & & s & & - & $\mathrm{R}$ & & & & R & & 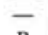 & & & & & - \\
\hline & & M & - & - & $\mathrm{F}$ & - & - & 1 & 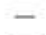 & - & 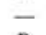 & & A & - & - & & $\mathrm{R}$ & 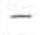 & - & & F & - \\
\hline $11 \mathrm{H}-\mathrm{CC}$ & A & $\mathrm{M}$ & $\mathbf{R}$ & - & $\mathrm{R}$ & $\mathrm{R}$ & - & K & - & - & $\mathbf{R}$ & $\mathrm{R}$ & A & $\mathbf{R}$ & $\mathrm{F}$ & - & - & - & - & $\mathrm{R}$ & $\mathbf{R}$ & - \\
\hline & & $\mathrm{N}$ & $\mathbf{R}$ & - & 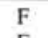 & 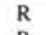 & $R_{2}$ & - & - & - & - & - & & - & & & - & & & $\mathrm{R}$ & & - \\
\hline & & 1 & $\mathrm{R}$ & & - & s & & & & & & - & & & $\mathrm{F}$ & & & & & 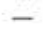 & & - \\
\hline & & N & 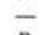 & & - & s & & & & 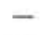 & - & - & A & & $\mathbf{R}$ & & & & & - & & - \\
\hline & & $\mathrm{M}$ & $\mathrm{R}$ & - & $\mathrm{R}$ & $\mathrm{R}$ & & 1 & & - & $\mathrm{R}$ & - & A & " & - & & & & - & - & & . \\
\hline $13 \mathrm{H}-5,60$ & A & $\mathrm{M}$ & $\mathrm{F}$ & - & - & $\mathrm{F}$ & $\mathrm{K}$ & $\mathrm{r}$ & - & - & - & - & A & - & $\mathrm{R}$ & $\mathrm{R}$ & - & - & - & $\mathbf{R}$ & $\mathrm{R}$ & - \\
\hline & & & & & $\mathrm{F}$ & $\mathbf{R}$ & & $\mathrm{R}$ & - & - & - & - & & & $\mathbf{R}$ & & & & & $R$ & & - \\
\hline & & $\mathrm{N}$ & $\mathrm{F}$ & - & 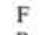 & $\mathrm{F}$ & $\mathbf{R}$ & & & - & $\pi$ & 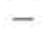 & & F & $\mathrm{s}$ & & & & & & & - \\
\hline & & N & F & & R & $\mathrm{F}$ & & & - & - & 5 & - & & 1 & $\mathrm{~F}$ & & & & 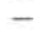 & 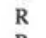 & F & - \\
\hline & & N & - & & - & R & - & 1 & - & - & a & 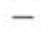 & A & $\mathrm{R}$ & - & & - & & - & $\mathbf{R}$ & , & 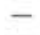 \\
\hline $15 \mathrm{H}-\mathrm{CC}$ & . & $\mathrm{M}$ & - & - & $\mathrm{R}$ & $\mathrm{R}$ & - & $\mathrm{K}$ & - & - & $\mathrm{F}$ & - & $\mathrm{A}$ & $\mathbf{R}$ & $\mathrm{R}$ & - & - & - & - & - & $\mathrm{R}$ & - \\
\hline & A & N & $R$ & - & $n$ & $\mathrm{~F}$ & - & t & - & - & - & - & A & $\mathrm{R}$ & - & & - & & - & - & $\mathrm{F}$ & - \\
\hline $16 \mathrm{H}-\mathrm{CC}$ & & $\mathrm{M}$ & $\mathbf{R}$ & - & $\mathrm{R}$ & - & - & . & - & - & $\mathrm{F}$ & 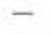 & A & n & $\mathbf{R}$ & - & - & - & - & - & R & - \\
\hline
\end{tabular}

Note: Abundance: $\mathrm{A}=$ abundant, $\mathrm{C}=$ common, $\mathrm{F}=$ few, $\mathrm{R}=$ rare; preservation: $\mathrm{G}=$ good, $\mathrm{M}=$ moderate, $\mathrm{P}=$ poor; lowercase letters indicate reworking. 
Table 10 (Continued).

\begin{tabular}{|c|c|c|c|c|c|c|c|c|c|c|c|c|c|c|c|c|c|c|}
\hline $\begin{array}{l}\text { हूँ } \\
\text { हूँ } \\
\text { है } \\
\text { हूँ } \\
\text { है }\end{array}$ & 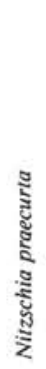 & 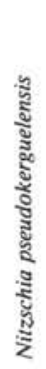 & 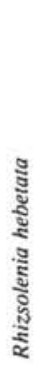 & 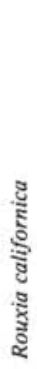 & 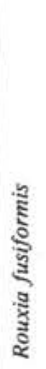 & 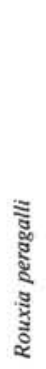 & 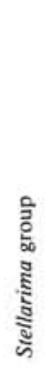 & 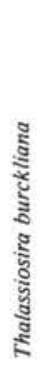 & 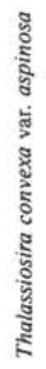 & 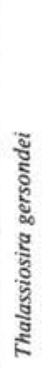 & 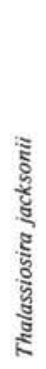 & 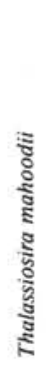 & 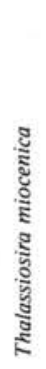 & 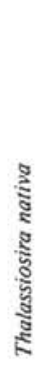 & 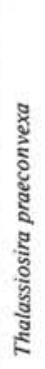 & $\begin{array}{l}- \\
\text { के } \\
\text { हूँ } \\
\text { है } \\
\text { है } \\
\text { है }\end{array}$ & 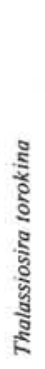 & 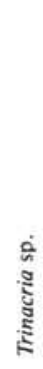 \\
\hline- & $\mathrm{F}$ & - & - & $\mathrm{R}$ & - & $\mathrm{F}$ & - & - & $F$ & - & - & - & $\mathrm{F}$ & $F$ & $\mathbf{R}$ & - & - & - \\
\hline $\mathrm{F}$ & $\mathrm{F}$ & $\mathbf{R}$ & - & $\mathrm{R}$ & - & $\mathrm{F}$ & - & - & - & $\mathrm{R}$ & $\mathbf{R}$ & - & - & $\mathrm{F}$ & $\mathrm{R}$ & - & - & - \\
\hline $\mathrm{R}$ & R & $\ddot{R}$ & - & $R$ & - & $\mathrm{F}$ & - & - & - & R & - & - & - & $\mathrm{F}$ & - & - & $R$ & - \\
\hline $\mathbf{R}$ & $\mathrm{C}$ & - & - & $\mathrm{R}$ & - & $\mathbf{R}$ & - & - & - & $\mathrm{F}$ & $R$ & - & - & $\mathrm{F}$ & $R$ & - & $\mathbf{R}$ & - \\
\hline$F$ & $\mathbf{R}$ & $\mathrm{R}$ & - & $\mathrm{R}$ & - & - & $R$ & - & - & $\mathrm{F}$ & $\mathbf{R}$ & - & - & R & - & - & $F$ & - \\
\hline$R$ & $\mathrm{R}$ & - & - & $\mathrm{R}$ & - & - & $R$ & - & - & $F$ & - & - & - & $R$ & - & - & $F$ & - \\
\hline- & - & - & $\mathbf{R}$ & $\mathrm{F}$ & - & - & R & - & - & $\mathrm{F}$ & - & - & - & $\ddot{R}$ & - & - & $\mathrm{F}$ & - \\
\hline- & - & - & $\widehat{F}$ & $\mathrm{R}$ & - & - & $\hat{F}$ & - & - & $\mathrm{F}$ & - & - & - & $\hat{R}$ & - & $\mathbf{R}$ & $\mathrm{F}$ & - \\
\hline- & - & - & - & $\mathrm{R}$ & - & - & - & - & - & $\mathrm{F}$ & - & - & - & $\hat{R}$ & - & $\ddot{-}$ & $\mathrm{F}$ & - \\
\hline- & - & - & - & $\mathrm{R}$ & - & - & $R$ & R & - & $\mathrm{F}$ & - & - & - & $\mathrm{F}$ & - & $\mathbf{R}$ & $\mathrm{F}$ & - \\
\hline- & $F$ & - & $\mathbf{R}$ & $\mathrm{R}$ & - & - & - & - & - & $R$ & - & - & - & - & - & $\mathbf{R}$ & $F$ & $\mathrm{R}$ \\
\hline- & R & - & $\ddot{R}$ & $\hat{F}$ & - & - & - & - & - & $\hat{F}$ & - & - & - & - & - & $\ddot{R}$ & R & $\mathrm{R}$ \\
\hline- & $\mathbf{R}$ & - & $F$ & C & - & - & - & $\mathbf{R}$ & - & $\mathrm{F}$ & - & - & - & $\mathrm{F}$ & - & - & $\ddot{F}$ & $\mathrm{R}$ \\
\hline- & $\vec{F}$ & - & $\mathrm{F}$ & $\mathrm{F}$ & - & - & - & - & - & $\mathrm{R}$ & - & - & - & $\mathrm{F}$ & 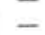 & $=$ & $\mathrm{F}$ & $\hat{\mathrm{F}}$ \\
\hline- & F & - & $\mathrm{F}$ & $\mathrm{F}$ & - & - & - & - & - & F & - & - & - & $\mathrm{F}$ & - & - & R & R \\
\hline- & $\mathrm{R}$ & - & $\mathbf{R}$ & - & - & - & $\mathrm{F}$ & $\mathrm{R}$ & - & $F$ & - & - & - & $\mathrm{F}$ & $=$ & - & $F$ & $R$ \\
\hline- & $\mathrm{F}$ & - & $F$ & $R$ & - & - & $\mathrm{R}$ & - & - & R & - & - & - & $\mathrm{F}$ & - & - & $\mathrm{F}$ & $\vec{F}$ \\
\hline- & $\mathrm{F}$ & - & - & $\vec{R}$ & - & - & $\hat{R}$ & - & - & $\mathrm{R}$ & - & - & - & $\mathrm{R}$ & - & - & R & $\mathrm{R}$ \\
\hline- & $\mathrm{R}$ & - & - & $\widehat{R}$ & - & - & $\ddot{R}$ & $\mathrm{R}$ & - & $\ddot{R}$ & - & - & - & $\vec{F}$ & - & - & $\mathbf{R}$ & $\mathrm{R}$ \\
\hline- & $\hat{F}$ & - & $\mathrm{F}$ & $\mathrm{R}$ & - & - & $\hat{R}$ & - & - & $\ddot{R}$ & - & - & - & $\mathrm{R}$ & - & - & $\ddot{R}$ & $\hat{R}$ \\
\hline- & $F$ & - & R & $\mathrm{R}$ & - & - & - & - & - & $\mathrm{R}$ & - & $\mathrm{R}$ & - & $F$ & - & - & $R$ & $\mathbf{R}$ \\
\hline- & $\mathrm{F}$ & - & $\mathrm{F}$ & $\hat{R}$ & - & - & $\bar{R}$ & $\bar{R}$ & - & R & - & $\ddot{\mathrm{F}}$ & - & - & - & - & $\hat{R}$ & - \\
\hline- & $\mathbf{R}$ & - & 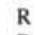 & $\mathrm{R}$ & - & - & $R$ & - & - & - & - & $\mathrm{R}$ & - & - & - & - & $\mathbf{R}$ & - \\
\hline- & $\mathrm{F}$ & - & $\mathbf{R}$ & $\mathbf{F}$ & - & - & - & - & - & 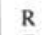 & - & $\mathbf{R}$ & - & $R$ & - & - & - & $\mathrm{R}$ \\
\hline- & $\mathrm{F}$ & - & $F$ & $R$ & - & - & $R$ & - & - & $\hat{F}$ & - & R & - & $\mathrm{R}$ & - & - & - & $\mathrm{R}$ \\
\hline- & $R$ & - & $\mathrm{F}$ & $F$ & - & $\overline{-}$ & - & - & - & $\mathbf{R}$ & - & $R$ & - & $\mathbf{R}$ & - & - & $F$ & - \\
\hline- & $\mathrm{R}$ & - & $\mathrm{F}$ & $R$ & 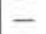 & - & $R$ & - & - & $\mathrm{R}$ & - & $\mathrm{F}$ & & $\mathrm{R}$ & - & - & $\mathrm{F}$ & $\mathrm{F}$ \\
\hline- & R & - & $R$ & - & - & - & $R$ & - & - & 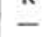 & - & $\mathrm{F}$ & - & $\hat{R}$ & - & - & R & $R$ \\
\hline- & $\vec{F}$ & - & $\ddot{R}$ & - & - & $\mathbf{R}$ & $\hat{F}$ & - & - & - & - & $c$ & - & - & - & - & $\hat{R}$ & $\mathrm{~F}$ \\
\hline- & R & - & $\mathrm{R}$ & $\mathrm{R}$ & - & $\mathrm{R}$ & $\mathrm{R}$ & $R$ & - & - & - & $\mathrm{F}$ & - & - & - & - & $\hat{R}$ & $\mathrm{R}$ \\
\hline- & $\mathrm{F}$ & - & $\mathbf{R}$ & $\mathrm{R}$ & - & - & $R$ & $\mathrm{~F}$ & - & - & - & $R$ & - & - & - & $R$ & $R$ & $\mathbf{R}$ \\
\hline- & $\mathbf{R}$ & - & $\widehat{R}$ & - & - & 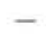 & $\hat{R}$ & - & - & $F$ & - & $\hat{R}$ & - & - & - & - & $\mathbf{F}$ & $R$ \\
\hline- & $\vec{F}$ & - & $\ddot{\mathrm{R}}$ & - & - & - & - & - & - & R & - & $\widehat{R}$ & - & - & - & - & $\mathrm{R}$ & $\mathrm{R}$ \\
\hline- & $\mathrm{F}$ & - & $\mathrm{R}$ & - & - & - & $\mathrm{R}$ & - & - & R & - & $\mathrm{F}$ & - & - & - & $\mathrm{R}$ & $\hat{R}$ & - \\
\hline- & $\mathbf{R}$ & - & $\mathrm{F}$ & - & - & - & $\mathrm{F}$ & - & - & $\ddot{R}$ & - & $\mathrm{R}$ & - & - & - & - & $\mathrm{R}$ & - \\
\hline- & $\mathrm{R}$ & - & $\mathrm{F}$ & - & - & - & $\mathrm{R}$ & - & - & $R$ & - & $F$ & - & $R$ & - & $\mathrm{R}$ & $\mathrm{R}$ & $R$ \\
\hline- & - & - & $F$ & - & $1-$ & - & R & - & - & R & - & $\mathrm{R}$ & - & $\mathbf{R}$ & - & - & R & $\mathrm{F}$ \\
\hline- & $\mathbf{R}$ & - & $\mathrm{F}$ & - & $L_{-}$ & - & - &.- & - & - & - & $\vec{F}$ & - & $\ddot{R}$ & - & $\mathrm{F}$ & - & $\mathrm{R}$ \\
\hline- & $\mathrm{R}$ & - & $\mathrm{F}$ & - & $\mathrm{R}$ & - & $\mathrm{R}$ & $\therefore$ & - & $\mathrm{F}$ & - & $\mathrm{F}$ & - & $\mathbb{R}$ & - & $\mathrm{F}$ & - & $\mathrm{F}$ \\
\hline- & $\mathrm{F}$ & - & - & - & - & - & $R$ & - & - & $\mathrm{F}$ & - & $\mathrm{F}$ & - & $\vec{R}$ & - & $R$ & - & $\mathrm{F}$ \\
\hline- & - & - & $\mathbf{R}$ & - & - & - & $F$ & - & - & $F$ & - & $F$ & - & - & - & $\mathbf{R}$ & - & $\mathbf{R}$ \\
\hline- & $\mathbf{R}$ & - & $\vec{F}$ & - & - & - & $\mathrm{R}$ & - & - & $F$ & - & $\mathrm{F}$ & - & $\mathrm{F}$ & - & $\ddot{R}$ & - & $\mathbf{R}$ \\
\hline- & $\mathbf{R}$ & - & $\mathrm{F}$ & - & $R$ & 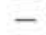 & $\mathrm{F}$ & - & - & - & - & - & - & $\mathrm{R}$ & - & $\mathrm{F}$ & - & F \\
\hline & - & - & $\mathrm{F}$ & - & $\ddot{-}$ & - & $\mathrm{F}$ & - & - & $\mathrm{R}$ & - & - & - & $\mathrm{R}$ & - & $\mathrm{F}$ & - & $\mathrm{R}$ \\
\hline- & $\mathbf{R}$ & - & $\mathrm{F}$ & - & - & - & $\mathrm{F}$ & - & - & $\ddot{R}$ & - & - & - & - & - & $\mathrm{F}$ & - & $\mathrm{R}$ \\
\hline- & $\mathrm{R}$ & - & - & - & - & - & $R$ & - & - & $R$ & - & - & - & $\mathbf{R}$ & - & $F$ & - & - \\
\hline . & - & - & $\mathrm{F}$ & - & $D$ & - & $\hat{R}$ & - & - & $\vec{F}$ & - & 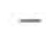 & - & $\ddot{-}$ & - & $\mathbf{R}$ & - & $\mathrm{R}$ \\
\hline- & $R$ & - & $\mathrm{F}$ & - & - & - & $\hat{R}$ & $R$ & - & $\mathrm{F}$ & - & - & - & $\mathrm{R}$ & - & $\ddot{R}$ & - & $\mathrm{F}$ \\
\hline- & $\mathbf{R}$ & - & $R$ & - & - & - & $\mathbf{R}$ & K & - & $\mathrm{F}$ & - & - & - & $\mathrm{R}$ & - & $\mathbf{R}$ & - & $\mathrm{F}$ \\
\hline- & $R$ & - & $F$ & - & - & - & $F$ & - & - & $R$ & - & - & - & $R$ & - & - & - & $F$ \\
\hline- & - & - & $\mathrm{F}$ & - & - & - & $R$ & - & - & $R$ & - & - & - & - & - & - & - & $\mathbf{R}$ \\
\hline & - & & $\mathrm{F}$ & - & & & $\ddot{R}$ & - & & $\ddot{R}$ & - & - & - & - & - & $\mathbf{R}$ & - & $\mathrm{R}$ \\
\hline- & - & - & $\mathrm{F}$ & - & - & - & $\hat{F}$ & - & - & R & - & - & - & - & - & 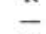 & - & $\mathrm{R}$ \\
\hline- & $\mathbf{R}$ & - & $\mathrm{F}$ & - & - & - & $\mathbf{R}$ & - & - & R & - & - & - & $R$ & - & $\mathbf{R}$ & - & $\mathrm{R}$ \\
\hline- & - & - & $\mathrm{F}$ & - & - & - & $\mathrm{F}$ & - & - & $\hat{R}$ & - & - & - & - & - & $\mathbf{R}$ & - & $\mathrm{R}$ \\
\hline- & $R$ & - & $F$ & - & - & - & $R$ & $R$ & - & $R$ & - & - & - & $\mathbf{R}$ & - & - & - & $\mathbf{R}$ \\
\hline & $\mathbf{R}$ & & $\mathrm{R}$ & - & & & - & & - & $\mathrm{F}$ & & - & & - & 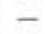 & $\mathrm{F}$ & - & $\mathbf{R}$ \\
\hline-7 & $\vec{F}$ & - & $\mathrm{R}$ & - & - & - & - & - & - & $\mathrm{F}$ & - & - & - & - & - & $\mathrm{F}$ & - & $\hat{R}$ \\
\hline- & $\mathbf{R}$ & - & $\overrightarrow{\mathrm{F}}$ & - & - & - & $\mathbf{R}$ & - & - & $R$ & - & - & - & $\mathbf{R}$ & - & $\mathrm{R}$ & - & $\mathrm{R}$ \\
\hline- & - & - & $\mathrm{F}$ & - & - & - & $\mathrm{F}$ & - & - & - & - & - & - & - & - & $\mathbf{R}$ & - & $\mathbf{R}$ \\
\hline- & $\mathrm{R}$ & - & $F$ & - & - & - & - & - & - & - & - & - & - & - & - & $\mathbf{R}$ & - & $\mathrm{R}$ \\
\hline- & - & - & $\mathrm{F}$ & - & - & - & $\mathbf{R}$ & - & - & - & - & - & - & - & - & $\ddot{R}$ & - & $\mathrm{R}$ \\
\hline
\end{tabular}




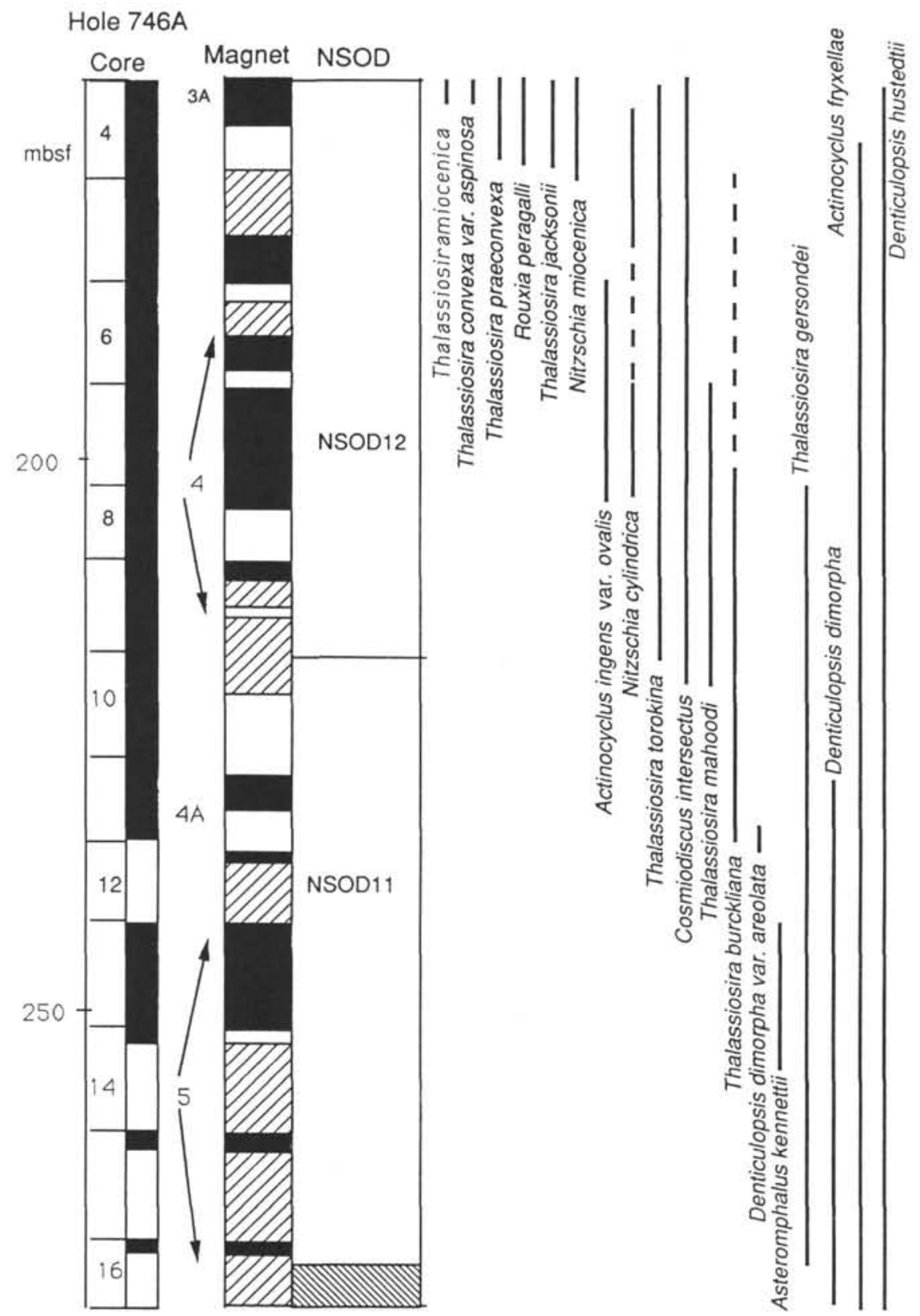

Figure 7. Stratigraphic recovery (Barron, Larsen, et al., 1989), paleomagnetics (H. Sakai, pers. comm., 1989), assigned zones, and the range of selected species from Hole 746A. The width of a range bar indicates relative abundance (see tables for specifics), and dashes indicate sporadic occurrences of an individual species for a given stratigraphic interval. Ruled area at base of diatom zone column indicates an unzoned interval.

Paleomagnetic and biostratigraphic results from Sites 744 through 746 provide an integrated Quaternary through Oligocene chronostratigraphy for testing the synchroneity of individual biostratigraphic events. The diatom assemblage observed from these sequences can be divided into two distinct categories. The Quaternary and Pliocene assemblage is characterized primarily by species endemic to the Southern Ocean, indicating the influence of the Antarctic Polar Front in this region and limited communication with surface waters from the lower latitudes. The Miocene and Oligocene assemblage generally is composed of cosmopoli- $\tan$ species, although species endemic to the Southern Ocean do occur in the middle Miocene interval.

\section{ACKNOWLEDGMENTS}

Special thanks are given to Doris Cooley, Polly Trant, and Judy Duke for valuable assistance in preparing the manuscript. We thank Rainer Gersonde and Lloyd Burckle for providing prepublication versions of their Leg 113 contribution. Sample preparation and photography were completed with the aid of So-Hung Kang, Al Mahood, and Lisa Meng. Funding for this 
Sample

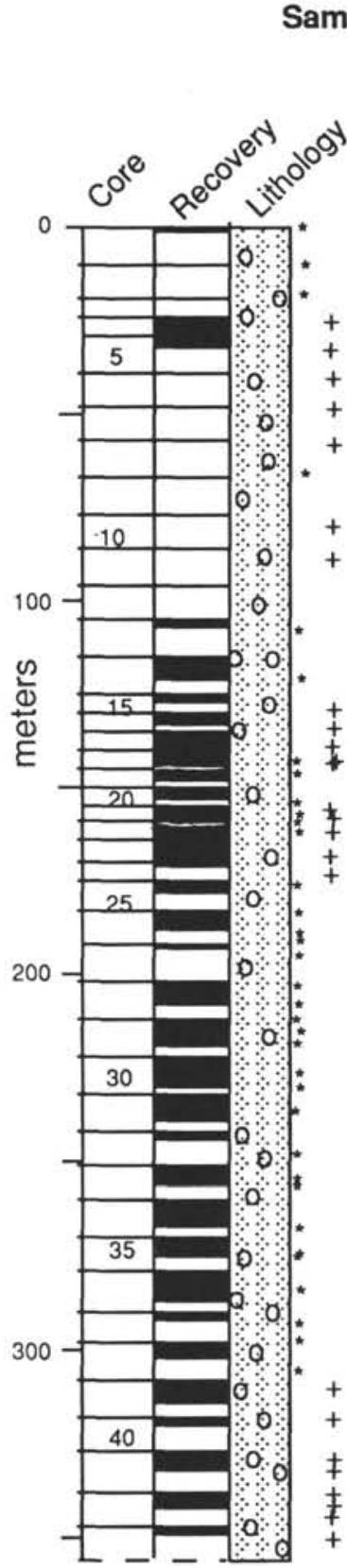

Age

Indicator species
Strontium

Dates

Ma

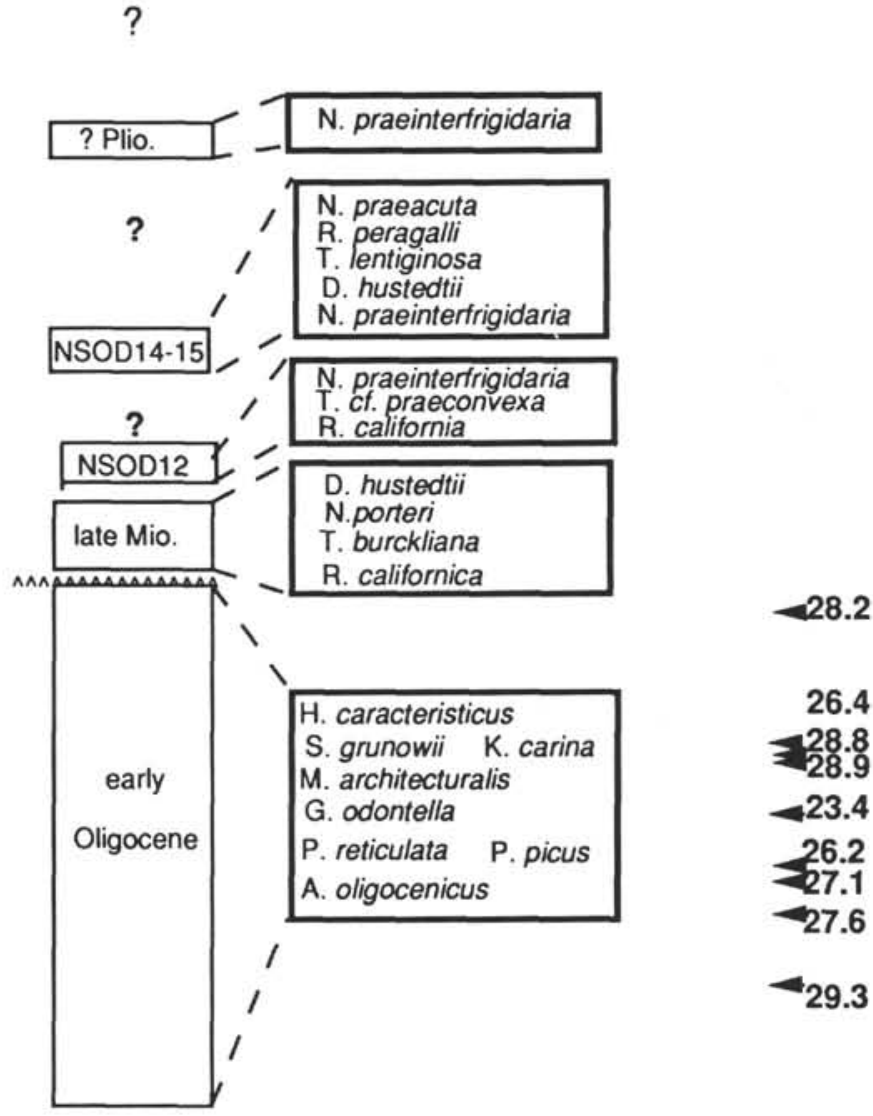

Figure 8. Stratigraphic $\log$ and lithology of sediments recovered from the upper $350 \mathrm{~m}$ of Hole 739A. * indicates samples in which no diatoms were observed; + indicates samples containing diatoms. Age and indicator species are also shown. $\wedge \wedge \wedge$ indicates a stratigraphic break in the record.

project was provided by USSAC. Samples were provided by the National Science Foundation. Robert Oscarson of the U.S. Geological Survey assisted in scanning electron microscope studies. This manuscript benefited from the reviews of David Harwood, Elizabeth Fourtanier, and an anonymous reviewer.

\section{REFERENCES}

Akiba, F., 1985. Middle Miocene to Quaternary diatom biostratigraphy in the Nankai trough and Japan trench, and modified lower Miocene through Quaternary diatom zones for middle-to-high latitudes of the North Pacific. In Kagami, H., Karig, D. E., Coulbourn, W. T., et al., Init. Repts. DSDP, 87: Washington (U.S. Govt. Printing Office), 393-481.
Akiba, F., and Yanagisawa, Y., 1985. Taxonomy, morphology and phylogeny of the Neogene diatom zonal marker species in the middle-tohigh latitudes of the North Pacific. In Kagami, H., Karig, D. E., Coulbourn, W. T., et al., Init. Repts. DSDP, 87: Washington (U.S. Govt. Printing Office), 483-554.

Baldauf, J. G., 1985. A high resolution late Miocene-Pliocene diatom biostratigraphy for the eastern equatorial Pacific. In Mayer, L., Theyer, F., et al., Init. Repts. DSDP, 85: Washington (U.S. Govt. Printing Office), 457-475.

Baldauf, J. G., and Barron, J. A., 1980. Actinocyclus ingens var. nodus: a new stratigraphically useful diatom of the circum-North Pacific. Micropaleontology, 26:103-110.

1982. Diatom biostratigraphy and paleoecology of the type section of the Luisian stage, central California, Micropaleontology, 28:59-84. 
Baldauf, J. G., and Monjanel, A.-L., 1989. An Oligocene diatom biostratigraphy for the Labrador Sea: DSDP Site 112 and ODP Hole 647A. In Srivastava, S. P., Arthur, M., Clement, B., et al., Proc. ODP, Sci. Results, 105: College Station, TX (Ocean Drilling Program), 323347.

Barker, P. F., Kennett, J. P., et al., 1988. Proc. ODP, Init. Repts., 113: College Station, TX (Ocean Drilling Program).

Barron, J. A., 1980. Lower Miocene to Quaternary diatom biostratigraphy of Leg 57, off Northeastern Japan, Deep Sea Drilling Project. In von Huene, R., Nasu, N., et al., Init. Repts. DSDP, 56, 57 (Pt. 2): Washington (U.S. Govt. Printing Office), 641-685.

1981. Late Cenozoic diatom biostratigraphy and paleoceanography of the middle-latitude eastern North Pacific, Deep Sea Drilling Project Leg 63. In Yeats, R. S., Haq, B. U., et al., Init. Repts. DSDP, 63: Washington (U.S. Govt. Printing Office), 507-535.

1983. Latest Oligocene through early middle Miocene diatom biostratigraphy of the eastern tropical Pacific. Mar. Micropaleontol., 7:487-515.

1985a. Late Eocene to Holocene diatom biostratigraphy of the equatorial Pacific Ocean, Deep Sea Drilling Project Leg 85. In Mayer, L., Theyer, F., et al., Init. Repts. DSDP, 85: Washington (U.S. Govt. Printing Office), 413-456.

1985b. Miocene to Holocene planktonic diatoms. In Bolli, H. M., Saunders, J. B., and Perch-Nielsen, K. (Eds.), Plankton Stratigraphy: Cambridge (Cambridge Univ. Press), 763-809.

in press. Neogene diatom datum levels in the equatorial and North Pacific. In Saito, T., and Ishizaki, K. (Eds.), The Centenary of Japanese Micropaleontology: Tokyo (Univ. Tokyo Press).

Barron, J. A., and Baldauf, J. G., 1986. Diatom stratigraphy of the lower Pliocene part of the Sisquoc Formation, Harris Grade section, California. Micropaleontology, 32:357-371.

Barron, J., Larsen, B., et al., 1989. Proc. ODP, Init. Repts. 119: College Station, TX (Ocean Drilling Program).

Berggren, W. A., Kent, D. V., Flynn, J. J., and Van Couvering, J. A., 1985. Cenozoic geochronology. Geol. Soc. Am. Bull., 96:1407-1418.

Bukry, D., and Foster, J. H., 1974. Silicoflagellate zonation of Upper Cretaceous to lower Miocene deep sea sediments. J. Res. U.S. Geol. Surv., 2:303-310.

Burckle, L. H., 1972. Late Cenozoic diatom zones from the eastern equatorial Pacific. Nova Hedwigia Beih., 39:217-246.

1978. Early Miocene to Pliocene diatom datum level for the equatorial Pacific. Geol. Res. Dev. Cent., Rep. Indones., Spec. Publ., $1: 25-44$.

Burckle, L. H., Clarke, D. B., and Shackleton, N. J., 1978. Isochronous last-abundant-appearance datum (LAAD) of the diatom Hemidiscus karstenii in the sub-Antarctic. Geology, 6:243-246.

Castracane, A. F., 1886. Report on the Scientific Results of the Voyage of H.M.S. Challenger During the Years 1873-1876. Botany (vol. 2): London (Her Majesty's Stationery Office).

Ciesielski, P. F., 1983. The Neogene and Quaternary diatom biostratigraphy of subantarctic sediments, Deep Sea Drilling Project Leg 71. In Ludwig, W. J., Krasheninnikov, V. A., et al., Init. Repts. DSDP, 71 (Pt. 2): Washington (U.S. Govt. Printing Office), 635-666.

Ciesielski, P. F., 1985. Middle Miocene to Quaternary diatom biostratigraphy of Deep Sea Drilling Project Site 594, Chatham Rise, southwest Pacific. In Kennett. J. P., von der Borch, C. C., et al., Init. Repts. DSDP, 90: Washington (U.S. Govt. Printing Office), 863-885.

Ciesielski, P. F., Kristoffersen, Y., et al., 1988. Proc. ODP, Init. Repts., 114: College Station, TX (Ocean Drilling Program).

Donahue, J. G., 1970. Pleistocene diatoms as climatic indicators in North Pacific sediments. Mem. Geol. Soc. Am., 126:121-138.

Dumont, M. P., Baldauf, J. G., and Barron, J. A., 1986. Thalassiosira praeoestrupii-a new diatom species for recognizing the Miocene/Pliocene Epoch boundary in coastal California. Micropaleontology, 32:372-377.

Dzinoridze, R. N., Jousé, A. P., Koroleva-Golikova, G. S., Kozlova, G. E., Nagaeva, M. G., Petrushevskaya, M. G., and Strelnikova, N. I., 1978. Diatom and radiolarian Cenozoic stratigraphy, Norwegian Basin; DSDP Leg 38. In Talwani, M., Udintsev, G., et al., Init. Repts. DSDP, Supplement to vols. $38,39,40$, and 41 : Washington (U.S. Govt. Printing Office), $289-428$.
Fenner, J., 1978. Cenozoic diatom biostratigraphy of the equatorial and southern Atlantic Ocean. In Perch-Nielsen, K., Supko, P. R., et al., Init. Repts. DSDP, 39 (Suppl.): Washington (U.S. Govt. Printing Office), 491-624.

1984. Eocene-Oligocene planktic diatom stratigraphy in the low latitudes and the high southern latitudes. Micropaleontology, 342 .

1985. Late Cretaceous to Oligocene planktonic diatoms. In Bolli, H. M., Saunders, J. B., and Perch-Nielsen, K. (Eds.), Plankton Stratigraphy: Cambridge (Cambridge Univ. Press), 713-762.

Fenner, J., Schrader, H. J., and Wienigk, H., 1976. Diatom phytoplankton studies in the southern Pacific Ocean, composition and correlation to the Antarctic Convergence and its paleoecological significance. In Hollister, C. D., Craddock, C., et al., Init.Repts. DSDP, 35: Washington (U.S. Govt. Printing Office), 757-813.

Fryxell, G. A., Prasad, A.K.S.K., and Fryxell, P. A., in press. Eucampia antarctica (Castracane) Mangin (Bacillariophyta): complex nomenclature and taxonomic history. Taxon.

Fryxell, G. A., Sims, P. A., and Watkins, T. P., 1986: Azpeitia (Bacillariophyceae): related genera and promorphology. Syst. Bot. Monographs, 13:1-74.

Gersonde, R., 1990. Taxonomy and morphostructure of Neogene diatoms from the Southern Ocean, ODP Leg 113. In Barker, P. F., Kennett, J. P., et al., Proc. ODP, Sci. Repts., 113: College Station, TX (Ocean Drilling Progarm), 791-802.

, in press. Taxonomy and morphostructure of Pliocene diatoms from the Maud Rise (Antarctic Ocean). Polarforschung.

Gersonde, R., and Burckle, L. H., 1990. Neogene diatom biostratigraphy of ODP Leg 113, Weddell Sea (Antarctic Ocean). In Barker, P. F., Kennett, J. P., et al., Proc. ODP, Sci. Results, 113: College Station, TX (Ocean Drilling Program), 761-789.

Gombos, A. M., Jr., 1977. Paleogene and Neogene diatoms from the Falkland Plateau and Malvinas Outer Basin: Leg 36, Deep Sea Drilling Project. In Barker, P., Dalziel, I.W.D., et al., Init. Repts. DSDP, 36: Washington (U.S. Govt. Printing Office), 575-687.

Gombos, A. M., Jr., and Ciesielski, P. F., 1983. Late Eocene to early Miocene diatoms from the southwest Atlantic. In Ludwig, W. J., Krasheninnikov, V. A., et al., Init. Repts. DSDP, 71 (Pt. 2): Washington (U.S. Govt. Printing Office), 583-684.

Grunow, A., 1884. Die Diatomeen von Franz-Josefs-Land. Denkschr. Kaiserlichen Akad. Wiss., Wien, Mathem.-Naturw. Classe, 49:5-117.

Hajòs, M., 1976. Upper Eocene and lower Oligocene diatomaceae, archaeomonadaceae, and silicoflagellates in southwestern Pacific sediments. In Hollister, C. D., Craddock, C., et al., Init. Repts. DSDP, 35: Washington (U.S. Govt. Printing Office), 817-884.

Hanna, G., 1932. The diatoms of Sharktooth Hill, Kern County, California, Proc. Calif. Acad. Sci., Fourth Ser., 20:161-263.

Harwood, D. M. 1986a. Diatom biostratigraphy and paleoecology with a Cenozoic history of antarctic ice sheets [Ph.D. dissert.]. Ohio State Univ., Columbus.

1986b. Diatoms. In Barrett, P. J. (Ed.), Antarctic Cenozoic History from the MSSTS-1 Drillhole, McMurdo Sound. DSIR Bull. N.Z., 237:69-107.

1989. Siliceous microfossils. In Barrett, P. J. (Ed.), Antarctic Cenozoic History from the CIROS-1 Drillhole, McMurdo Sound. DSIR Bull. N.Z., 245:67-97.

Harwood, D. M., Barrett, P. J., Edwards, A. R., Rieck, H. J., and Webb, P.-N., 1989. Biostratigraphy and chronology. In Barrett, P. J. (Ed.), Antarctic Cenozoic History from the CIROS-1 Drillhole, McMurdo Sound. DSIR Bull. N.Z., 245:231-239.

Jousé, A. P., 1961. Miocene and Pliocene marine diatoms from the Far East. Botan. Materialy Otdela Sporovych Rastenij Botan. Inst. Akad. NAUK, SSSR, 16:59-70.

1974. Diatoms in the Oligocene-Miocene biostratigraphic zones of the tropical areas of the Pacific Ocean. Nova Hedwigia Beih., 45:333-364.

Kanaya, T., 1959. Miocene diatom assemblages from the Onnagawa Formation and their distribution in correlative formations in northeast Japan. Sci. Rep. Tohoku Univ. Ser. 2, 30:1-130.

Keller, G., and Barron, J. A., 1987. Paleodepth distribution of Neogene deep-sea hiatuses. Paleoceanography, 2:697-713. 
Kellogg, D. E., and Kellogg, T. B., 1986. Diatom biostratigraphy of sediment cores beneath the Ross Ice Shelf. Micropaleontology, 32:74-79.

Koizumi, I., and Tanimura, Y., 1985. Neogene diatom biostratigraphy of the middle latitude western North Pacific, Deep Sea Drilling Project Leg 86. In Heath, G. R., Burckle, L. H., et al., Init. Repts. DSDP, 86: Washington (U.S. Govt. Printing Office), 269-300.

McCollum, D. W., 1975. Diatom stratigraphy of the southern ocean. In Hayes, D. E., Frakes, L. A., et al., Init. Repts., DSDP, 28: Washington (U.S. Govt. Printing Office), 515-571.

Schrader, H.-J., 1973. Cenozoic diatoms from the northeast Pacific, Leg 18. In Kulm, L. D., von Huene, R., et al., Init. Repts. DSDP, 18: Washington (U.S. Govt. Printing Office), 673-797.

1974. Cenozoic marine planktonic diatom stratigraphy of the tropical Indian Ocean. In Fisher, R. L., Bunce, E. T., et al., Init. Repts. DSDP, 24: Washington (U.S. Govt. Printing Office), 887-967.

1976. Cenozoic planktonic diatom biostratigraphy of the Southern Pacific Ocean. In Hollister, C. D., Craddock, C., et al., Init. Repts. DSDP, 35: Washington (U.S. Govt. Printing Office), 605-672.

Schrader, H.-J., and Fenner, J., 1976. Norwegian Sea Cenozoic diatom biostratigraphy and taxonomy. In Talwani, M., Udintsev, G., et al., Init. Repts. DSDP, 38: Washington (U.S. Govt. Printing Office), 921-1099.

Simonsen, R., 1979. The diatom system: ideas on phylogeny. Bacillaria, 2:9-71.

Simonsen, R., and Kanaya, T., 1961. Notes on the marine species of the diatom genus Denticula Kutz. Int. Rev. Gesamten Hydrobiol., 46: 498-513.

Sims, P. A., Fryxell, G. A., and Baldauf, J. G., 1989. Critical examination of the diatom genus Azpeitia: species useful as stratigraphic markers for the Oligocene/Miocene Epochs. Micropaleontology, 35:293-307.

Tauxe, L., Tucker, P., Petersen, N. P., and LaBrecque, J. L., 1984. Magnetostratigraphy of Leg 73 sediments. In Hsü, K. J., LaBrecque, J. L., et al., Init. Repts. DSDP, 73: Washington (U.S. Govt. Printing Office), 609-621.

Weaver, F. M., and Gombos, A. M., 1981. Southern high-latitude diatom biostratigraphy. In Warme, T. E., Douglas, R. G., and Winterer, E. L. (Eds.), The Deep Sea Drilling Project: A Decade of Progress: Spec. Publ. Soc. Econ. Paleontol. Mineral., 32:445-470.

Yanagisawa, Y., and Akiba, A., in press. Taxonomy and phylogeny of the three marine diatom genera Crucidenticula, Denticulopsis, and Neodenticula. Chishitsu Chosasho Geppo.

\section{Date of initial receipt: 9 October 1989}

Date of acceptance: 18 June 1990

Ms 119B-135

\section{APPENDIX}

Taxonomic Citation of Diatom Taxa and Important Synonyms

The reader is referred to Akiba (1985), Akiba and Yanagisawa (1985), Barron (1981, 1983, 1985a, 1985b), Barron and Baldauf (1986), Fryxell et al. (1986), Koizumi and Tanimura (1985), Schrader (1973), and Sims et al. (1989) for systematics and illustrations.

\section{New Taxa \\ Actinocyclus fryxellae Barron $\mathrm{n} . \mathrm{sp}$.}

$$
\text { (Pl. 1, Figs. 1, 2, 4) }
$$

Cestodiscus sp. 2 sensu Schrader, 1976, pl. 12, fig 7.

Actinocyclus octonarius Ehrenberg sensu Harwood, 1986a, pl. 19, fig. 16 (not $12,13)$.

Description. Valves are circular, 13-58 $\mu \mathrm{m}$ in diameter, with most 25-35 $\mu \mathrm{m}$. Areolae are radially arranged in fasciculated rows: areola vary from seven to 10 in $10 \mu \mathrm{m}$ in the center to 10 to 14 in $10 \mu \mathrm{m}$ near the margin depending on valve size. Areolae are loosely concentrated in the valve center, where typically a single central areolae is surrounded by a hyaline field. The prominent marginal hyaline fields ( $2-4 \mu \mathrm{m}$ in length), which terminate single rows of areolae, give the impression of rays (PI. 1, Figs. 1,2); each marginal hyaline field appears to coincide with a marginal tube or internal marginal process ( $\mathrm{Pl}$. 1, Fig. 2). The pseudonodulus is not distinct and is located on the margin, adjacent to one of the marginal hyaline fields (Pl. 1, Fig. 2). The striated margin is rarely preserved (Pl. 1, Fig. 2).
2).

Holotype. Sample 119-746A-7H-6, 60-62 cm, USNM 444983 (PI. 1, Fig.

Stratigraphic range. Late Miocene (10.0-6.1 Ma).

Derivation of name. Named in honor of Greta Fryxell, Dept. of Oceanography, Texas A\&M University, College Station, Texas.

Thalassiosira gersondei Barron n. sp.

(Pl. 2, Figs. 1, 2; Pl. 3, Figs. 1, 3, 5, 6; Pl. 5, Fig. 4)

Description. The robust circular valve is $35-76 \mu \mathrm{m}$ in diameter. The areolae are radially arranged in straight rows forming a fasciculate pattern of six to eight areolae in $10 \mu \mathrm{m}$ with no decrease in areolae size from valve center to margin. Primary rows of areolae extend from the margin to the center and are separated by six to eight secondary rows that terminate at varying distances from the margin and form the fasciculate pattern. The prominent margin, 4-6 $\mu \mathrm{m}$ wide, consists of an inner ring of marginal processes (four to five in 10 $\mu \mathrm{m}$ ), a finely aerolaed mantle (eight to 10 areolae in $10 \mu \mathrm{m}$ ), and an outer striated ring (PI. 3, Figs. 1, 6). The labiate process was not observed. Five to six central strutted processes occur arranged in a trifultate pattern (Pl. 3, Figs. $3,5)$. $1)$.

Holotype. Sample 119-746A-7H-6, 60-62 cm, USNM 444984 (PI. 2, Fig.

Isotype. Sample 119-746A-7H-6, 60-62 cm, USNM 444985 (PI. 2, Fig. 2).

Stratigraphic range. Late Miocene (9.7-7.4 Ma).

Derivation of name. Named in honor of Rainer Gersonde, Alfred Wegener Institute for Polar and Marine Research, Bremerhaven, Germany.

Thalassiosira mahoodii Barron n. sp.

(Pl. 2, Figs. 3-5; Pl. 3, Figs. 2, 4; Pl. 4, Figs. 1-6)

Description. The circular valve is $42-74 \mu \mathrm{m}$ in diameter and typically displays yellow brown to light brown interference colors under lower magnifications $(\times 500)$. The valves are flat to slightly convex, commonly with a slightly depressed center. Areolae are radially arranged in an irregular fasciculate pattern, 15-17 in $10 \mu \mathrm{m}$, with no apparent change in size between valve center and margin. Numerous short marginal processes (six to eight in $10 \mu \mathrm{m}$ ) are located on the distal portion of the gently sloping valve mantle (Pl. 4, Fig. 2; Pl. 3, Figs. 2, 4). The single labiate process is near the valve margin ( $\mathrm{Pl} .4$, Fig. 6); the marginal processes are interrupted for approximately $10 \mu \mathrm{m}$ near the labiate process (PI. 3, Figs. 2,4). The external opening of the labiate process is ventral to the marginal processes (Pl. 3, Fig. 4). No processes are visible externally at the valve center (PI. 4, Fig. 3), but the two openings observed internally at the valve's center (Pl. 4, Fig. 4) may be the dissolved remanents of processes.

Holotype. Sample 119-746A-7H-6, 60-63 cm, USNM 444986 (PI. 2, Fig. 4).

Isotype. USNM 444987 and 444988 (Pl. 2, Figs. 3, 5).

Stratigraphic range. Late Miocene (7.8-6.8 Ma).

Derivation of name. Named in honor of Al Mahood, California Academy of Sciences, San Francisco, California.

\section{Flora List}

Actinocyclus actinochilus (Ehrenberg) Simonsen. Synonym: Charcotia actinochilus (Ehrenberg) Hustedt; Gombos, 1976, pl. 1, fig. 8.

Actinocyclus fryxellae Barron n. sp., Baldauf and Barron, herein (see preceding).

Actinocyclus ingens Rattray, Baldauf and Barron, 1982, p. 68., pl. 1, figs. 6-10. (PI. 5, Fig. 2)

Actinocyclus ingens var, nodus Baldauf in Baldauf and Barron, 1980, p. 104, pl. 1, figs. 5-9.

Actinocyclus ingens var. ovalis Gersonde; Gersonde and Burckle, 1990, pl. 5, figs. 4-5. (Pl. 5, Fig. 1)

Actinocyclus tsugaruensis Kanaya, 1959, pl. 8, figs. 5-8.

Actinoptychus senarius Ehrenberg; Baldauf and Monjanel, 1989, pl. 1, fig. 7. Actinoptychus thumii Hanna, 1932, p. 171, pl. 4, figs. 3, 4.

Asterolampra acutiloba Forti; Gombos and Ciesielski, 1983, p. 600, pl. 1, fig. 8.

Asterolampra schmidtii Hajòs, 1976, p. 827, pl. 21, fig. 6; Gombos and Ciesielski, 1983, p. 600, pl. 2, figs. 1-3, pl. 4, figs. 9, 10.

Asterolampra vulgaris var. hyalina Gombos, in Gombos and Ciesielski, 1983, p. 606 , pl. 1, figs. 3,9 . 
Table 11. Occurrence of selected diatoms species observed from Hole 739C.

\begin{tabular}{|c|c|c|c|c|c|c|c|c|c|c|c|c|c|c|c|c|c|c|c|c|c|c|c|c|c|c|}
\hline $\begin{array}{l}\text { Core, section, } \\
\text { interval (cm) }\end{array}$ & 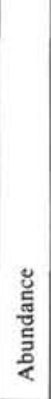 & 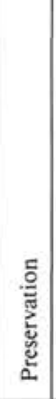 & 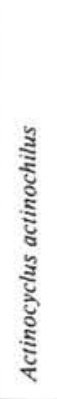 & 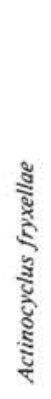 & 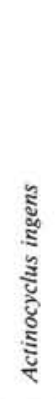 & 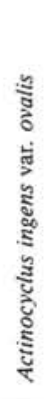 & 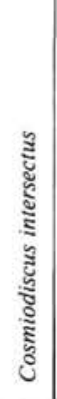 & 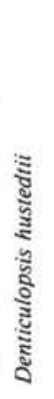 & 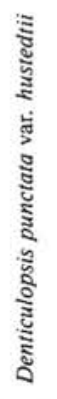 & 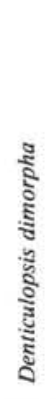 & 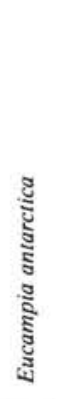 & 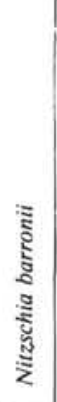 & 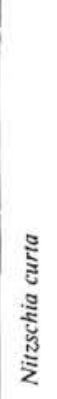 & 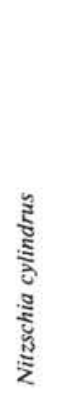 & 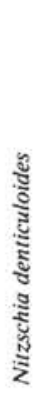 & 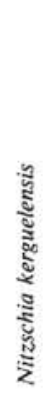 & 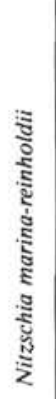 & 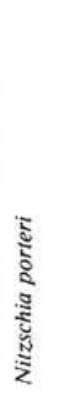 & 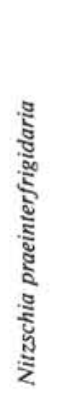 & 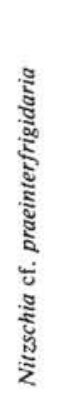 & 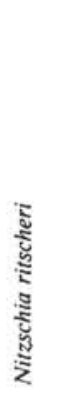 & 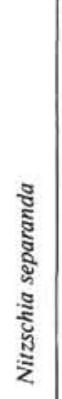 & 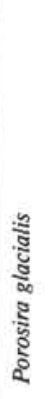 & 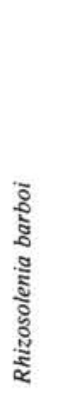 & 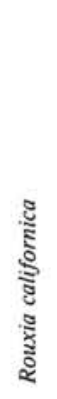 & 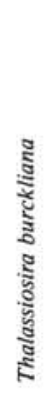 \\
\hline \multicolumn{27}{|l|}{$119.739 \mathrm{C}$. } \\
\hline $1 \mathrm{H}-1,84$ & c & G & - & - & - & - & - & - & - & - & $\mathrm{F}$ & - & $\bar{x}$ & $\mathrm{C}$ & - & $\mathrm{F}$ & - & - & - & - & $\bar{x}$ & - & $\mathrm{R}$ & - & - & - \\
\hline $1 \mathrm{H} \cdot \mathrm{CC}$ & c & G & 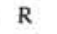 & - & - & - & - & - & - & - & $F$ & $\mathbf{R}$ & $F$ & $\mathrm{~F}$ & - & $\mathrm{F}$ & - & - & - & - & $\mathrm{F}$ & - & $\mathbf{R}$ & - & - & - \\
\hline $2 \mathrm{H} \cdot \mathrm{CC}$ & $\mathbf{R}$ & $P$ & - & - & - & - & - & - & - & - & - & - & - & - & - & $\mathrm{R}$ & - & - & - & - & - & - & - & - & - & - \\
\hline $8 \mathrm{H}-\mathrm{CC}$ & $\mathrm{R}$ & P & - & - & - & - & - & - & - & - & - & - & - & - & - & - & - & - & $r$ & - & - & - & - & - & - & - \\
\hline $10 \mathrm{H}-\mathrm{CC}$ & $\mathrm{R}$ & $\mathrm{P}$ & - & - & - & - & - & - & - & - & - & - & - & - & - & - & - & - & - & - & - & - & - & - & - & - \\
\hline $12 \mathrm{H}-\mathrm{CC}$ & $\mathrm{F}$ & $\mathrm{M}$ & $R$ & - & - & - & - & - & - & - & - & $\mathrm{R}$ & $R$ & $\mathrm{~F}$ & - & $\mathrm{R}$ & - & - & - & $\bar{p}$ & $R$ & - & - & - & - & - \\
\hline $13 \mathrm{H}-\mathrm{CC}$ & R & $\mathrm{P}$ & - & - & - & - & - & $r$ & - & - & - & - & - & $\mathbf{R}$ & - & - & - & - & - & $\mathbf{R}$ & - & - & - & - & - & - \\
\hline $14 \mathrm{H}-\mathrm{CC}$ & $\mathrm{R}$ & $\mathrm{P}$ & - & - & - & - & - & $r$ & - & - & - & - & - & - & - & - & - & - & $\mathbf{R}$ & - & - & - & - & - & - & - \\
\hline $17 \mathrm{H}-\mathrm{CC}$ & R & $P$ & - & - & - & - & - & $r$ & - & - & - & - & - & - & - & - & - & - & - & - & - & - & - & - & - & - \\
\hline $18 \mathrm{H}-\mathrm{CC}$ & $\mathrm{C}$ & $\mathrm{M}$ & - & - & $\mathrm{R}$ & - & - & $\mathrm{C}$ & $\mathrm{R}$ & $\mathbf{R}$ & - & - & - & - & $\mathbf{R}$ & - & - & $\mathbf{R}$ & - & - & - & - & - & - & - & $\mathrm{R}$ \\
\hline $19 \mathrm{H}-2$ & C & G & - & $R$ & $R$ & $R$ & $\mathrm{~F}$ & C & - & - & $\mathrm{R}$ & - & - & - & - & - & - & - & - & $R$ & - & - & - & - & $R$ & $\mathrm{R}$ \\
\hline $19 \mathrm{H}-\mathrm{CC}$ & C & $\mathrm{M}$ & - & $\mathrm{R}$ & - & - & $\mathrm{F}$ & F & & 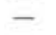 & - & - & - & - & $k$ & - & $\pi$ & $\mathrm{R}$ & - & $\mathrm{R}$ & - & - & - & - & R & $\mathrm{R}$ \\
\hline $20 \mathrm{H}-2,80$ & $\mathrm{~F}$ & M & - & - & - & - & - & $\mathrm{R}$ & - & - & - & - & - & - & - & - & $\mathrm{R}$ & - & - & - & - & - & - & - & $\mathbf{R}$ & - \\
\hline $20 \mathrm{H}-\mathrm{CC}$ & $\mathrm{R}$ & $P$ & - & - & - & - & - & $\mathrm{R}$ & - & - & - & - & - & - & - & - & - & - & - & - & - & - & - & - & - & - \\
\hline $22 \mathrm{H}-\mathrm{CC}$ & $\mathrm{F}$ & $\mathrm{M}$ & - & - & $\mathrm{R}$ & - & - & $\mathrm{F}$ & - & - & - & - & - & $\mathbf{R}$ & $\mathbf{R}$ & - & $\mathbf{R}$ & $\mathbf{R}$ & - & - & - & - & - & - & - & $\mathrm{R}$ \\
\hline $23 \mathrm{H} \cdot \mathrm{C}$ & $\mathrm{R}$ & $P$ & - & - & - & - & - & $R$ & - & - & - & - & - & - & - & - & - & - & - & - & - & - & - & - & - & - \\
\hline$-1,68$ & $\mathrm{R}$ & P & - & - & - & - & - & - & - & - & - & - & - & - & - & $\mathrm{R}$ & - & - & - & - & - & - & - & - & - & - \\
\hline $4 . C C$ & E & $P$ & - & - & - & - & - & - & - & - & - & - & - & - & - & - & - & - & - & - & - & - & - & - & - & - \\
\hline $26 \mathrm{H}-1,70$ & 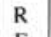 & $\mathrm{P}$ & - & - & - & - & - & - & - & - & - & - & - & - & - & - & - & - & - & - & - & - & - & - & - & - \\
\hline $26 \mathrm{H}-3,76$ & $\mathrm{~F}$ & $\mathrm{P}$ & - & - & - & - & - & - & - & - & - & - & - & - & - & - & - & - & - & - & - & - & - & - & - & - \\
\hline $26 \mathrm{H}-\mathrm{CC}$ & C & M & - & - & - & - & - & - & - & - & - & - & - & - & - & - & - & - & - & - & - & - & - & - & - & - \\
\hline 2 & $F$ & $\mathrm{P}$ & & - & & & & & - & & - & - & & - & & - & & - & - & - & - & & - & - & - & - \\
\hline $29 \mathrm{H}-4,58$ & 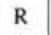 & $\mathrm{P}$ & - & - & - & - & - & - & - & - & - & - & - & - & 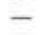 & $\mathrm{R}$ & -1 & - & - & - & - & & - & - & - & - \\
\hline $30 \mathrm{H}-1$ & A & G & - & - & - & - & - & - & - & - & - & - & - & - & - & - & - & - & - & - & - & - & - & - & - & - \\
\hline $30 \mathrm{H}-2,80$ & $\mathrm{~F}$ & $\mathrm{M}$ & - & - & - & - & - & - & - & - & - & - & - & - & - & - & - & - & - & - & - & - & - & - & - & - \\
\hline $30 \mathrm{H}-4,80$ & $R$ & $\mathrm{P}$ & - & - & - & - & - & - & - & - & - & - & - & - & - & - & - & - & - & - & - & - & - & - & - & - \\
\hline & R & P & & - & & 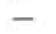 & - & & - & 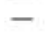 & - & - & 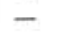 & 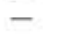 & & 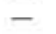 & & - & - & & - & & - & - & $\pi$ & - \\
\hline $32 \mathrm{H}-1,96$ & $\mathrm{~F}$ & $\mathrm{P}$ & - & - & - & - & - & - & - & - & - & - & - & - & - & - & - & - & - & - & - & - & - & - & - & - \\
\hline & C & M & - & - & - & - & - & - & - & - & - & - & - & - & - & - & - & - & - & - & - & - & - & - & - & - \\
\hline $33 \mathrm{H}-4,80$ & $\mathrm{~F}$ & $\mathrm{M}$ & - & - & - & - & - & - & - & - & - & - & - & - & - & - & - & - & - & - & - & - & - & - & - & - \\
\hline $33 \mathrm{H}-2$ & C & M & - & - & - & - & - & & - & - & - & - & - & - & - & - & - & - & - & - & - & - & - & - & - & - \\
\hline A-CC & C & M & - & - & - & - & - & - & - & - & - & - & - & - & - & - & - & - & - & - & - & - & - & - & - & - \\
\hline $35 \mathrm{H}-2,80$ & $\mathbf{R}$ & $\mathrm{P}$ & - & - & - & - & - & - & - & - & - & - & - & - & - & - & - & - & - & - & - & - & - & - & - & - \\
\hline $35 \mathrm{H}-4,32$ & F & $\mathrm{P}$ & - & - & - & - & - & - & - & - & - & - & - & - & - & - & - & - & - & - & - & - & - & - & - & - \\
\hline $35 \mathrm{H}-\mathrm{CC}$ & $\mathrm{R}$ & $P$ & - & - & - & - & - & - & - & - & - & - & - & - & - & - & - & - & - & - & - & - & - & - & - & - \\
\hline $36 \mathrm{H}-\mathrm{CC}$ & C & P & & - & - & - & - & & - & & - & & & & & & & - & & & & & - & & - & - \\
\hline $37 \mathrm{H}-1,60$ & $\mathrm{R}$ & P & - & - & - & - & - & - & - & - & - & - & - & - & - & - & - & - & - & - & - & - & - & - & - & - \\
\hline $37 \mathrm{H}-\mathrm{CC}$ & F & $\mathrm{P}$ & - & - & - & - & - & - & - & - & - & - & - & - & - & - & - & - & - & - & - & - & - & - & - & - \\
\hline $38 \mathrm{H}-1,26$ & $\mathrm{~F}$ & M & - & - & - & - & - & - & - & - & - & - & - & - & - & - & - & - & - & - & - & - & - & - & - & - \\
\hline $38 \mathrm{H}-\mathrm{CC}$ & $\mathrm{F}$ & $\mathrm{P}$ & - & - & - & - & - & - & - & - & - & - & - & - & - & - & - & - & - & - & - & - & - & - & - & - \\
\hline
\end{tabular}

Species occurrence is grouped: Actinocyclus actinochilus through Thalassiosira torokina are characteristic of the Neogene/Quaternary; Asteromphalus oligocenicus through Thalassiosira hydra are characteristic of the Paleogene. Abundance: $\mathrm{A}=$ abundant, $\mathrm{C}=$ common, $\mathrm{F}=$ few, $\mathrm{R}=$ rare; preservation: $\mathrm{G}=$ good, $\mathrm{M}=\operatorname{moderate}, \mathrm{P}=$ poor; lowercase letters indicate reworking.

Asteromphalus kennettii Gersonde; Gersonde and Burckle, 1990, pl. 5, fig. 6. Synonym: Asteromphalus sp. cf. A. symmetricus Schrader and Fenner, 1976; Harwood et al., in press, pl. 4, fig. 3.

Asteromphalus oligocenicus Schrader et Fenner, 1976, pl. 21, figs. 8, 13, 14; pl. 28 , fig. 1.
Azpeitia apiculata Sims et al., 1989. Synonym: Coscinodiscus tuberculatus Greville sens Burckle, 1978; Barron, 1985b, fig. 1.

Azpeitia endoi-A. tabularis. Includes Azpeitia tabularis Fryxell et $\mathrm{Sims}$ in Fryxell et al., 1986, p. 16, figs. XIV, XV, XXX-1, and A. endoi (Kanaya) Sims et Fryxell in Fryxell et al., 1986, p. 16. 


\begin{tabular}{|c|c|c|c|c|c|c|c|c|c|c|c|c|c|c|c|c|c|c|c|c|c|c|c|c|c|c|c|}
\hline 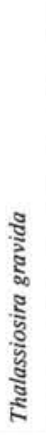 & 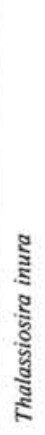 & 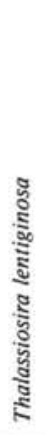 & 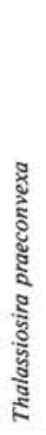 & 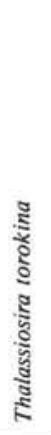 & 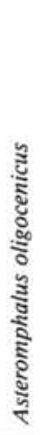 & 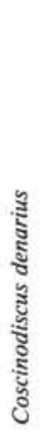 & 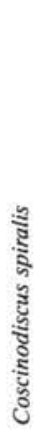 & 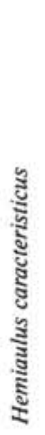 & 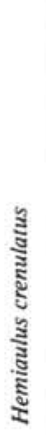 & 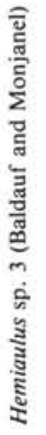 & 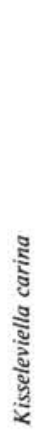 & 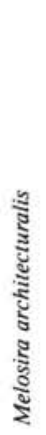 & 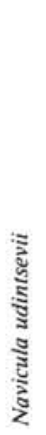 & 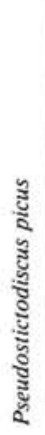 & 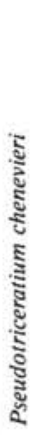 & 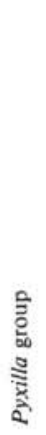 & 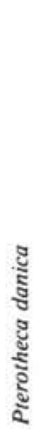 & 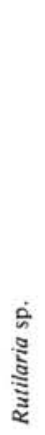 & 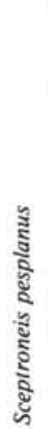 & 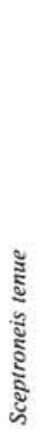 & 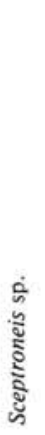 & 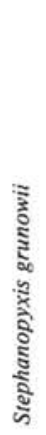 & 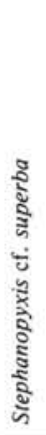 & 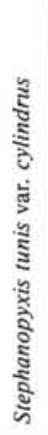 & 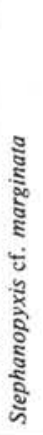 & 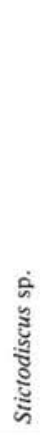 & 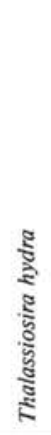 \\
\hline $\mathbf{F}$ & $\mathrm{R}$ & - & - & - & - & - & - & - & - & - & - & - & - & - & - & - & - & - & - & - & - & - & - & - & - & - & - \\
\hline $\mathrm{F}$ & $\mathrm{R}$ & $\mathbf{R}$ & - & - & - & - & - & - & - & - & - & - & - & - & - & - & - & - & - & - & - & - & - & - & - & - & - \\
\hline $\mathrm{R}$ & - & - & - & - & - & - & - & - & - & - & - & - & - & - & - & - & - & - & - & - & - & - & - & - & - & - & - \\
\hline- & - & - & - & - & - & - & - & - & - & - & - & - & - & - & - & - & - & - & - & - & - & - & - & - & - & - & - \\
\hline $\mathbf{R}$ & - & - & - & - & - & - & - & - & - & - & - & - & - & - & - & - & - & - & - & - & - & - & - & - & - & - & - \\
\hline $\mathrm{R}$ & - & $\mathbf{R}$ & - & - & - & - & - & - & - & - & - & - & - & - & - & - & - & - & - & - & - & - & - & - & - & - & - \\
\hline- & - & $\mathrm{R}$ & - & $\mathbf{R}$ & - & - & - & - & - & - & - & $\pi$ & - & $\overline{-}$ & & - & 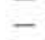 & - & - & & - & & - & - & - & - & - \\
\hline- & - & - & - & - & - & - & - & - & - & - & - & - & - & - & & - & - & - & - & - & - & - & - & - & - & - & - \\
\hline- & - & - & - & - & - & - & - & - & - & - & - & - & - & - & - & - & - & - & - & - & - & - & - & - & - & - & - \\
\hline- & - & - & - & - & - & - & - & - & - & - & - & - & - & - & - & - & - & - & - & - & - & - & - & - & - & - & - \\
\hline- & $\mathrm{R}$ & - & $\mathrm{R}$ & 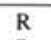 & - & - & - & - & - & - & - & - & - & - & - & - & - & - & - & - & - & - & - & - & - & - & - \\
\hline- & - & - & - & $n$ & 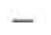 & - & - & - & - & 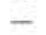 & 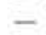 & 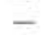 & - & - & & - & - & & - & & - & & - & - & - & - & - \\
\hline- & - & - & - & $\mathrm{F}$ & - & - & - & - & - & - & - & - & - & - & - & - & - & - & - & & - & - & - & - & - & - & - \\
\hline- & - & - & - & - & - & - & - & - & - & - & - & - & - & - & - & - & - & - & - & - & - & - & - & - & - & - & - \\
\hline- & - & - & - & $\mathrm{R}$ & - & - & - & - & - & - & - & - & - & - & - & - & - & - & - & - & - & - & - & - & - & - & - \\
\hline- & - & - & - & - & - & - & - & - & - & - & - & - & - & - & - & - & - & - & - & - & - & - & - & - & - & - & - \\
\hline- & - & - & - & - & 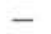 & - & 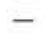 & - & - & & - & . & & - & & $\mathrm{R}$ & & & - & & - & $R$ & - & - & - & - & - \\
\hline- & - & - & - & - & - & - & - & - & - & & 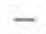 & - & 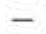 & - & 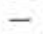 & 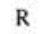 & - & - & - & & - & $R$ & - & - & - & - & - \\
\hline- & - & - & - & - & - & - & - & - & - & & - & - & - & - & - & $\ddot{R}$ & - & - & - & - & - & $\ddot{R}$ & - & - & - & - & - \\
\hline- & - & - & - & - & - & - & - & - & - & - & - & - & - & - & - & $\mathrm{R}$ & - & - & - & - & - & $\mathrm{R}$ & - & - & - & - & - \\
\hline- & - & - & - & - & - & $R$ & - & $\mathrm{F}$ & - & $R$ & - & - & - & - & $R$ & $\mathrm{~F}$ & - & - & - & - & - & $\mathrm{R}$ & $\mathbf{R}$ & $\mathrm{R}$ & - & - & $R$ \\
\hline- & - & 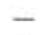 & 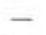 & & & - & & - & 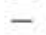 & 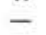 & $\mathrm{R}$ & & - & - & & - & & & - & & & & - & - & - & - & - \\
\hline- & - & - & - & - & - & - & - & - & - & - & - & - & - & - & - & $\mathrm{R}$ & - & - & - & - & - & $\mathrm{R}$ & - & - & - & - & - \\
\hline- & - & - & - & - & $\mathrm{R}$ & - & $\mathbf{R}$ & $F$ & $\mathrm{R}$ & 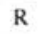 & $\mathrm{F}$ & $\mathrm{R}$ & - & $\mathbf{R}$ & & $\mathrm{F}$ & $R$ & $\mathrm{R}$ & - & & - & $C$ & $\mathbf{R}$ & $\mathbf{R}$ & - & - & - \\
\hline- & - & - & - & - & - & - & - & - & - & - & - & - & - & - & - & $\mathrm{R}$ & - & - & - & - & - & R & - & - & - & - & - \\
\hline- & - & - & - & - & - & - & - & - & - & - & - & - & - & - & $=$ & - & - & - & - & - & - & $\mathrm{R}$ & - & - & - & - & - \\
\hline 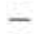 & & 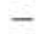 & 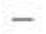 & & & 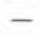 & - & - & - & & - & 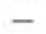 & - & - & & $\mathrm{R}$ & & & - & & - & $\mathrm{R}$ & - & - & - & - & - \\
\hline- & - & - & - & - & - & - & - & - & - & & - & - & $=$ & $\overline{-}$ & $R$ & $\hat{\mathbf{R}}$ & 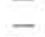 & - & - & - & $\bar{R}$ & $\hat{\mathrm{R}}$ & - & - & - & - & - \\
\hline- & - & - & & - & 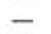 & - & & $\mathrm{R}$ & & & $\mathrm{R}$ & & - & - & & $\vec{F}$ & & & - & & - & $\mathrm{F}$ & - & $\mathbf{R}$ & $\mathrm{R}$ & 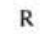 & - \\
\hline- & - & - & - & - & $\mathrm{R}$ & - & - & - & - & - & - & - & - & - & - & $\mathrm{F}$ & - & - & - & - & - & $\mathrm{R}$ & - & - & - & R & - \\
\hline- & & - & - & - & $R$ & - & $\mathrm{R}$ & $\mathrm{R}$ & $R$ & - & $\mathrm{F}$ & & & $\mathrm{R}$ & $F$ & $F$ & $\mathbf{R}$ & - & $\mathrm{R}$ & & - & $\mathrm{F}$ & - & $\mathrm{F}$ & - & $R$ & - \\
\hline- & - & - & - & - & - & - & - & - & - & & 0 & - & & $N$ & & $\mathrm{~F}$ & 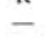 & & - & & - & $\mathrm{F}$ & - & $\mathrm{F}$ & $\mathrm{R}$ & $\underline{-}$ & - \\
\hline- & - & - & - & - & 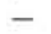 & - & & 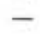 & & & 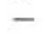 & & - & & - & - & & & - & & - & $\mathrm{R}$ & - & - & - & - & - \\
\hline- & - & - & - & - & - & - & - & - & - & 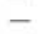 & - & - & - & - & $R$ & $\mathrm{R}$ & - & - & - & 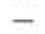 & - & $\mathrm{R}$ & - & - & - & - & - \\
\hline- & - & - & - & - & - & - & - & $\mathrm{R}$ & - & - & - & - & - & - & - & $\ddot{R}$ & - & - & - & - & - & $\ddot{R}$ & - & - & - & - & - \\
\hline- & - & - & - & - & - & - & - & $R$ & - & - & - & - & - & - & - & $\mathrm{R}$ & - & - & - & - & $R$ & $\mathbf{R}$ & - & $\mathrm{R}$ & - & - & - \\
\hline- & - & - & - & - & - & - & - & - & 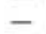 & & - & & & & & - & 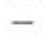 & & 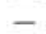 & & - & $\ddot{R}$ & - & - & - & - & - \\
\hline- & - & - & - & - & - & - & - & $\mathrm{R}$ & - & - & - & - & - & - & - & $R$ & - & - & - & 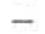 & - & $\mathrm{F}$ & $\mathrm{R}$ & - & - & $R$ & - \\
\hline- & - & - & - & - & - & - & - & $\mathrm{R}$ & $\mathrm{R}$ & $R$ & - & - & - & - & - & $\mathbf{R}$ & - & - & - & - & $\mathrm{R}$ & $\mathrm{F}$ & - & - & - & - & - \\
\hline- & - & - & - & - & - & - & - & - & - & - & - & - & - & - & - & - & - & - & - & - & - & - & - & - & - & - & - \\
\hline
\end{tabular}

Azpeitia nodulifer (A. Schmidt) Fryxell $e t$ Sims in Fryxell et al., 1986, p. 19, figs. XVII, XVIII-1, -2, -3, -5, XXX-3, -4. Synonym: Coscinodiscus nodulifer A. Schmidt; see Fryxell et al., 1986, for discussion.

Azpeitia oligocenica (Jousé) Sims in Fryxell et al., 1986, p. 16. Synonym: Coscinodiscus oligocenicus Jousé, 1974, p. 348, pl. 1, figs. 6-8; Coscinodiscus oligocenicus var. nodosus Jousé, 1974.

Azpeitia praenodulifer (Barron) Sims et Fryxell in Fryxell et al., 1986, p. 16. Synonym: Coscinodiscus praenodulifer Barron, 1983, p. 511, pl. III, figs. 9, 10; pl. IV, fig. 8.
Azpeitia salisburyana (Lohman) Sims in Sims et al., 1989. Synonym: Coscinodiscus salisburyanus Lohman, Barron, 1981, pl. 2, fig. 5.

Bogorovia veniamini Jousé, 1974, p. 351, pl. 4, figs. 1-3.

Cestodiscus antarctica Fenner, 1984, pl. 1, fig. 1.

Cestodiscus convexus Castracane, 1886, pl. 2, fig. 7; pl. 7, fig. 7 .

Cestodiscus parmula Castracane, 1886, pl. 7, fig. 5 .

Cestodiscus pulchellus Greville; Barron, 1985a, p. 780, fig. 10.15.

Cestodiscus reticulatus Fenner, 1984, pl. 1, fig. 10. Synonym: Coscinodiscus superbus Hardmann sensu Fenner, 1978, pl. 14, figs. 2-3. 
Table 12. Magnetostratigraphic calibration and the estimated age of diatom events observed from Sites 744 through 746 .

\begin{tabular}{|c|c|c|c|}
\hline Datum & $\begin{array}{c}\text { Hole 744B } \\
\text { (age, Ma) }\end{array}$ & $\begin{array}{l}\text { Hole } 745 \mathrm{~B} \\
\text { (age, Ma) }\end{array}$ & $\begin{array}{l}\text { Hole } 746 \\
\text { (age, Ma) }\end{array}$ \\
\hline LO $A$. ingens & & $\mathrm{C} 1 \mathrm{~N}-1(0.4-0.55)$ & \\
\hline LO C. elliptopora & & C1R-1 to C1N-2 $(0.75-1.0)$ & \\
\hline LOR. barboi & & $\mathrm{C} 1 \mathrm{R}-2$ to $\mathrm{C} 2 \mathrm{~N}-1$ (1.55-1.75) & \\
\hline LO C. kolbei & & $\mathrm{C} 2 \mathrm{~N}-1(1.75-1.9)$ & \\
\hline FO C. elliptopora & & C1R-2 to $\mathrm{C} 2 \mathrm{~N}-1(1.55-1.75)$ & \\
\hline LO C. vulnificus & & C2R-1 (2.2-2.3) & \\
\hline LO C. insignis & & C2R-1 (2.4-2.45) & \\
\hline LO N. weaveri & & $\mathrm{C} 2 \mathrm{~N}-1(1.75-1.9)$ & \\
\hline FO N. kerguelensis & & C2R-1 (1.9-2.0) & \\
\hline LO $N$. interfrigidaria & & C2R-1 (1.9-2.0) & \\
\hline FO C. vulnificus & & $\mathrm{C} 2 \mathrm{AN}-1(2.7-2.8)$ & \\
\hline FO N. weaveri & & C2AR-2 (3.1-3.2) & \\
\hline FO $R$. heteropolara & & C2AR-3 $(3.75-3.8)$ & \\
\hline FO $N$. barronii & & C3R-1 (4.0) & \\
\hline FO $N$. praeinterfrigidaria & & C3R-2 TO C3R-3 (4.34-4.55) & \\
\hline FO $T$. inura & & $\mathrm{C} 3 \mathrm{~N}-4(4.7-4.8)$ & \\
\hline FO $T$. oestrupii & & C $3 R-4(5.1-5.3)$ & \\
\hline LO $T$. miocenica & & & \\
\hline FO $T$. convexa var. aspinosa & & & $\mathrm{C} 3 \mathrm{AN}-2(5.8)$ \\
\hline FO R. peragalli & & & C3AR-2 (6.0) \\
\hline FO T. praeconvexa & & & C3AR-2 (6.0) \\
\hline FO T. jacksonii & & & C3AR-2 (6.0) \\
\hline LO A. fryxellae & & & C3AR-2 (6.1) \\
\hline FO N. miocenica & & & C3AR-2 (6.1) \\
\hline LO $T$. burckliana & & & C 3 AR-2 (6.2) \\
\hline LCO D. hustedtii & & & C3AR-2 (6.3) \\
\hline LO A. ingens var. ovalis & & & C3AR-3 (6.5) \\
\hline LO $T$. mahoodii & & & C4R-1 (6.8) \\
\hline FO N. marina-reinholdii & & & $\mathrm{C} 4 \mathrm{~N}-2-3(7.3)$ \\
\hline FO $A$. ingens var. ovalis & & & $\mathrm{C} 4 \mathrm{~N}-2-3(7.4)$ \\
\hline FO N. cylindrica & & & $\mathrm{C} 4 \mathrm{~N}-3(7.4)$ \\
\hline LO T. gersonde & & & $\mathrm{C} 4 \mathrm{~N}-3(7.4)$ \\
\hline FO $T$. torokina & & & C4R-3 (7.7) \\
\hline FO $T$. mahoodii & & & C4R-3 (7.8) \\
\hline FO $C$. intersectus & & & C4R-3 (7.8) \\
\hline LO $D$. hustedtii var. ovata & & & C $4 \mathrm{R}-3$ (7.9) \\
\hline FO T. burckliana & & & C4AN-2 (8.5) \\
\hline LO D. dimorpha var. areolata & & & C4AN-2 (8.4) \\
\hline LO A. kennettii & & & C4AR-2-3 (8.6-8.9) \\
\hline FO D. dimorpha var. areolata & C5N-1 (9.6) & & \\
\hline FO $A$. kennettii & C $5 \mathrm{~N}-1$ ( 9.6$)$ & & $\mathrm{C} 5 \mathrm{~N}-1(9.6)$ \\
\hline FO $D$, hustedtii var. ovalis & $\mathrm{C} 5 \mathrm{~N}-1$ (9.7) & & \\
\hline
\end{tabular}

Cestodiscus robustus Jousé, 1974; Baldauf and Monjanel, 1989, pl. 1, fig. 2. Coscinodiscus blysmos Barron, 1983, pl. 2, figs. 6, 7; pl. 6, fig. 7.

Coscinodiscus derarius A. Schmidt; Hajòs, 1976, pl. 7, figs, 4, 5.

Coscinodiscus elliptopora Donahue, 1970; Gombos, 1976, p. 592, pl. 3, figs. $1-3,6$; pl. 9, fig. 3 .

Coscinodiscus hajosiae Fenner, 1984, pl. 2, fig. 1. Synonym: Coscinodiscus spiralis Hajòs, 1976, p. 826, pl. 7, figs. 1-3.

Coscinodiscus marginatus Ehrenberg; Baldauf and Barron, 1982; Schrader, 1973, pl. 20, figs. $7,10,12,13$.

Coscinodiscus lewisianus Greville; Schrader, 1973, pl. 8, figs. 1-6, 10, 15.

Coscinodiscus rhombicus Castracane; Schrader and Fenner, 1976, pl. 21, figs. $1-3,5$.

Coscinodiscus vulnificus Gombos, 1976, p. 593, pl. 4, figs. 1-3, pl. 42, figs. $1-2$.

Cosmiodiscus insignis Jousé; McCollum, 1975, pl. 8, fig. 5.

Cosmiodiscus insignis f. triangula Jousé; Ciesielski, 1983, pl. 5, figs. 1-10.

Cosmiodiscus intersectus (Brun) Jousé, 1961, p. 68, pl. 2, figs. 9, 10; Gersonde and Burckle, 1990, pl. 4, fig. 13. (Pl. 6, Figs. 3, 6)

Crucidenticula kanayae Akiba et Yanagisawa, 1985, p. 486, pl. 1, figs. 3-8; pl. 3, figs. 1-6, 9, 11. Synonym: Denticula kanayae Akiba ex Barron, 1980, p. 672 , pl. 1 , figs. $26-28$.

Crucidenticula nicobarica (Grunow) Akiba et Yanagisawa, 1985, p. 486, pl. 1, fig. 9; pl. 2, figs. 1-7; pl. 5, figs. 1-9. Synonym: Denticula nicobarica Grunow; Schrader, 1973, pl. 1, figs. 31-35; Denticulopsis nicobarica (Grunow) Simonsen, 1979, p. 65. (Pl. 7, Fig. 8)

Crucidenticula paranicobarica Akiba et Yanagisawa, 1985, p. 26, fig. 7.
Denticulopsis dimorpha (Schrader) Simonsen emend. Akiba et Yanagisawa, 1985 , p. 488 , pl. 15 , figs. 1-25; pl. 16, figs. 1-11. Synonym: Denticula dimorpha Schrader, 1973, p. 704, pl. 1, figs. 37-46. (Pl. 7, Fig. 4)

Denticulopsis dimorpha var, areolata Yanagisawa et Akiba, in press. Synonym: Denticulopsis dimorpha sensu Akiba, 1985, pl. 27, figs. 1-2, 5-6, 10

Denticulopsis hustedtii (Simonsen et Kanaya) Simonsen; Akiba and Yanagisawa, 1985, p. 488, pl. 17, figs. 4-5, 7-23; pl. 18, figs. 1-10; pl. 19, figs. 1-5; pl. 7, figs. 16-29; pl. 9, figs. 1-9. Synonym: Denticula hustedti Simonsen et Kanaya 1961, p. 501, pl. 1, figs. 19-25. (Pl. 7, Fig. 1)

Denticulopsis hustedtii var.ovata (Schrader) Simonsen, 1979, p. 64. Synonym: Denticula hustedtii var. ovata Schrader, 1976, pl. 4, figs. 5, 6, 12 (14?,15?).

Denticulopsis hyalina (Schrader) Simonsen; Akiba and Yanagisawa, 1985, pl, 10, figs. 1-16; pl. 11, figs. 1-10; pl. 12, figs. 1-5. Synonym: Denticula hyalina Schrader, 1973, p. 704, pl. 1, figs. 12-22.

Denticulopsis lauta (Bailey) Simonsen; Akiba and Yanagisawa, 1985, p. 489 pl. 7, figs. 16-29; pl. 9, figs. 1-9. Synonym: Denticula lauta Bailey; Simonsen et Kanaya, 1961, p. 500, pl. 1, figs. 1-5. (PI. 7, Fig. 3)

Denticulopsis maccollumii Simonsen, 1979, p. 65. Synonym: Denticula antarctica McCollum; Schrader, 1976, p. 631, pl. 4, figs., 3, 22, 23, 25.

Denticulopsis praedimorpha Barron ex Akiba; Akiba and Yanagisawa, 1985 , p. 489, pl. 13, figs. 1-28; pl. 14, figs. 1-12. (PI. 7, Fig. 9)

Denticulopsis praelauta Akiba et Koizumi; Akiba and Yanagisawa, 1985, pl. 7 , figs. $1-15$; pl. 8 , figs. $1-9$.

Denticulopsis punctata Schrader, 1973, pl. 1, figs. 25-30; pl. 3, figs. 16, 17. Denticulopsis punctata var. hustedtii Schrader, 1973, pl. 1, figs. 23, 24.

Ethmodiscus sp. 
Table 12 (Continued).

\begin{tabular}{|c|c|c|c|}
\hline Datum & $\begin{array}{c}\text { Hole 744B } \\
\text { (age, Ma) }\end{array}$ & $\begin{array}{c}\text { Hole } 745 \mathrm{~B} \\
\text { (age, Ma) }\end{array}$ & $\begin{array}{r}\text { Hole } 746 \\
\text { (age, Ma) }\end{array}$ \\
\hline FO A. fryxellae & C5R-1 (10.5) & & \\
\hline LO D. praedimorpha & $\mathrm{C} 5 \mathrm{~N}-3(11.0-11.1)$ & & \\
\hline LO $N$. denticuloides & C5R-3 (11.4) & & \\
\hline FO D. dimorpha & C5AN-2 (11.9-12.0) & & \\
\hline \multirow[t]{2}{*}{ FO D. praedimorpha } & $\mathrm{C} 5 \mathrm{AN}-2$ to $\mathrm{C} 5 \mathrm{AN}-3(12.0-12.5)$ & & \\
\hline & Holes $744 \mathrm{~A} / 744 \mathrm{~B}$ & & \\
\hline LO C. nicobarica & C5AN-2 (11.9-12.0) & & \\
\hline LO N. grossepunctata & C5AN-2 (11.9-12.0) & & \\
\hline LO A. ingens var. nodus & C5AN-1 to C5AN-3 (12.0-12.5) & & \\
\hline FO N. denticuloides & C5ABN-1 (13.3-13.4) & & \\
\hline FCO D. hustedtii & C5AC-C5AD (14.0) & & \\
\hline FO D. hustedtii & C5AD-C5AD (14.2) & & \\
\hline FO $A$. ingens var. nodus & C5BN (15.0) & & \\
\hline FO $A$. ingens & $\operatorname{C5CN}(16.4)$ & & \\
\hline FO D, lauta & C5CR-3 (16.4) & & \\
\hline LO $N$. maleinterpretaria & $\operatorname{C5CN}(16.7)$ & & \\
\hline FO N. grossepunctata & $\mathrm{C} 5 \mathrm{CN}(16.7)$ & & \\
\hline FO D. maccollumii & C5CR-3 (17.2) & & \\
\hline FO C. kanayae & $\mathrm{C} 5 \mathrm{DN}-1(17.7-17.8)$ & & \\
\hline FO C. nicobarica & C5DN-1 (17.7-17.8) & & \\
\hline LO T. spumellaroides & $\mathrm{C} 6 \mathrm{~N}-1(20.0-20.1)$ & & \\
\hline FO $T$. fraga & C6R-1 to C6AN-1 $(20.3-20.4)$ & & \\
\hline FO T. spumellaroides & C6AAN-1 $(21.0-21.2)$ & & \\
\hline FO T. spinosa & C6CR-3 (24.0-21.5) & & \\
\hline LCO $R$. schraderi & C6AAN-1 (27.0-27.2) & & \\
\hline LO $L$. ornata & $\mathrm{C} 8 \mathrm{~N}(27.0-27.2)$ & & \\
\hline FO R. schraderi & $\mathrm{C} 8 \mathrm{~N}(27.0-27.2)$ & & \\
\hline FO $R$, gelida & $\mathrm{C} 8 \mathrm{~N}(27.4-27.6)$ & & \\
\hline FO $L$, ornata & C8N (27.4-27.6) & & \\
\hline FO B. veniamini & $\mathrm{C} 8 \mathrm{~N}-1(27.6-27.8)$ & & \\
\hline LO $H$. insignis & ? & & \\
\hline LO R. granda & ? & & \\
\hline LO A. schmidtii & $\mathrm{C} 11 \mathrm{R}-\mathrm{C} 12 \mathrm{~N}(30.6-31.2)$ & & \\
\hline FO $R$, vigilans & $\mathrm{C} 11 \mathrm{~N}(31.8-32.3)$ & & \\
\hline LO C. reticulatus & C11 (31.8-32.3) & & \\
\hline FO S. jouseana & $\mathrm{C} 12 \mathrm{~N}(32.4-32.7)$ & & \\
\hline LO $R$. oligocenica & $\mathrm{C} 12 \mathrm{R}(33.3-33.4)$ & & \\
\hline FO C. reticulatus & $\mathrm{C} 13 \mathrm{~N}(35.3)$ & & \\
\hline LO T. macroporum & $\mathrm{C} 13 \mathrm{~N}(35.4-35.6)$ & & \\
\hline FO A. schmidtii & $\mathrm{C} 13 \mathrm{~N}(35.6-35.7)$ & & \\
\hline LO $H$. caracteristicus & $\mathrm{C} 13 \mathrm{~N}(35.9-36.0)$ & & \\
\hline FO $R$. granda & $\mathrm{C} 13 \mathrm{~N}(35.7-35.9)$ & & \\
\hline
\end{tabular}

Note: $\mathrm{LO}=$ last occurrence, $\mathrm{FO}=$ first occurrence, $\mathrm{FCO}=$ first common occurrence, $\mathrm{LCO}=$ last common occurrence.

Eucampia antarctica Mangin; Fryxell et al., in press.

Goniothecium odontella Ehrenberg; Schrader and Fenner, 1976, pl. 6, figs. 1, 2,4 .

Hemiaulus caracteristicus Hajòs, 1976, pl. 15, fig. 10.

Hemiaulus incisus Hajòs, 1976, pl. 23, figs. 4-9.

Hemiaulus polycystinorum Ehrenberg; Baldauf and Monjanel, 1989, pl. 2, figs. $14-17$.

Hemiaulus polymorphus Grunow; Fenner, 1978, pl. 21, fig. 11; pl. 23, figs. 10, 11 ; pl. 22, fig. 13.

Hemiaulus pungens Grunow, 1884, pl. 5, fig. 56.

Hemiaulus tarus Gombos et Ciesielski, 1983, pl. 19, figs. 1-8.

Hemiaulus crenatus Greville, Baldauf and Monjanel, 1989, pl. 4, fig. 4.

Hemiaulus sp. 3 Baldauf and Monjanel, 1989, p. 341, pl. 3, fig. 11.

Hemidiscus cuneiformis Wallich; Barron, 1985a, p. 786, fig. 9.13.

Hemidiscus karstenii Jousé; Ciesielski, 1983, p. 656.

Lisitzinia ornata Jousé, Gombos and Ciesielski, 1983, pl. 18, figs. 1-4.

Lithodesmium reynoldsii Barron; Barron, 1980, pl. 4, fig. 10, Barron, 1985a, p. 786 , fig. 12.7 .

Melosira architecturalis Brun; Fenner, 1985, p. 734, figs. 12.4-12.5.

Navicula udintsevii Schrader et Fenner, 1976, p. 991, pl. 23, fig. 33; pl. 24, fig. 1; Gombos and Ciesielski, 1983, pl. 21, fig. 8.

Nitzschia barronii Gersonde, in press; Gersonde and Burckle, 1990, pl. 1, figs. 11-13. (Pl. 7, Fig. 14)

Nitzschia clementia Gombos; Gersonde and Burckle, 1990, pl. 2, figs. 22-23.
Nitzschia curta (Van Heurck) Hasle; Fenner et al., 1976, pl. 4, figs. 5-9.

Nitzschia cylindrica Burckle; Gersonde and Burckle, 1990, pl. 1, fig. 27. (Pl. 7, Fig. 10)

Nitzschia cylindrus (Grunow) Hasle; Fenner et al., 1976, pl. 4, figs. 10-15. Nitzschia denticuloides Schrader, 1976, pl. 3, figs. 7, 8, 10, 12, 18-24. (PI. 7, Fig. 2)

Nitzschia donahuensis Schrader, 1976, pl. 2, fig. 30. (Pl. 7, Fig. 6)

Nitzschia fossilis (Frenguelli) emend. Kanaya ex Schrader; Schrader, 1973, pl. 4, figs. 9-11, 24, 25 .

Nitzschia grossepunctata Schrader, 1976, pl. 3, figs. 1-4.

Nitzschia interfrigidaria McCollum, 1975, pl. 9, figs. 7-9.

Nitzschia januaria Schrader, 1976, pl. 2, figs. 25-29. (Pl. 5, Fig. 10)

Nitzschia kerguelensis (O'Meara) Hasle; Fenner et al., 1976, pl. 2, figs. 19-30.

Nitzschia maleinterpretaria Schrader, 1976, pl. 2, figs. 9, 11-19, 21, 24.

Nitzschia marina Grunow; Schrader, 1973, pl. 4, figs. 17-19. (Pl. 7, Fig. 11)

Nitzschia miocenica Burckle; Barron, 1985a, pl. 13, fig. 5. (Pl. 7, Fig. 13)

Nitzschia panduriformis Gregory; Fenner, 1978, pl. 32, figs. 27-29.

Nitzschia pliocena (Brun) Mertz; Akiba and Yanagisawa, 1985, pl. 40, figs. $1-7$.

Nitzschia porteri Frenguelli sensu Burckle, 1972; Barron, 1985a, pl. 13, fig. 7 .

Nitzschia praecurta Gersonde; Gersonde and Burckle, 1990, pl. 1, figs. 21-24. (PI. 7, Fig. 15)

Nitzschia praereinholdii Schrader, 1973, pl. 5, figs. 20, 23-26.

Nitzschia praeinterfrigidaria McCollum, 1975, pl. 10, fig. 1. (Pl. 7, Fig. 12) 
Table 13. Stratigraphic constraints of individual diatom events as a result of reexamining the stratigraphic sequences from DSDP Sites 266 and 278.

\begin{tabular}{|c|c|c|c|}
\hline Datum & $\begin{array}{c}\text { Age } \\
\text { (Ma) }\end{array}$ & Site 266 & Site 278 \\
\hline FO $T$. inura & 4.47 & Hiatus & $\begin{array}{c}9-4,99 / 10-1,80 \\
\text { Hiatus }\end{array}$ \\
\hline FO D. dimorpha var. areolata & 9.6 & & $9-6,54 / 10-1,54$ \\
\hline FO A. kennettii & 9.6 & & $10-1,54 / 11-6,53$ \\
\hline FO $D$. hustedtii var. ovata & 9.7 & & $9-6,54 / 10-1,54$ \\
\hline LCO D. dimorpha & 9.9 & $9-4,99 / 10-1,80$ & $11-6,53 / 12-1,53$ \\
\hline FO A. fryxellae & 10.5 & $10-1,80 / 10-3,77$ & $12-3,47 / 12-4,52$ \\
\hline LO D. praedimorpha & 11.0 & $10-4,40 / 10-5,30$ & $13-2,51 / 13-3,54$ \\
\hline LO N. denticuloides & 11.5 & $10-5,30 / 10-6,80$ & $13-6,53 / 14-2,53$ \\
\hline FO D. dimorpha & 11.9 & $11-4,90 / 11-5,30$ & $14-6,53 / 15-1,74$ \\
\hline FO D. praedimorpha & 12.6 & $11-4,90 / 11-5,30$ & $15-2,51 / 15-3,54$ \\
\hline LO C. nicobarica & 12.2 & $11-4,90 / 11-5,30$ & $14-6,53 / 15-1,74$ \\
\hline LO N. grossepunctata & 13.5 & & $16-5,50 / 16-6,50$ \\
\hline LO $A$. ingens var. nodus & 12.3 & & $16-4,53 / 16-6,52$ \\
\hline FO $N$. denticuloides & 13.5 & $12-2,113 / 13-1,95$ & $16-5,50 / 16-6,50$ \\
\hline FCO D. hustedtii & 14.0 & $12-2,113 / 13-1,95$ & $16-4,53 / 16-6,52$ \\
\hline FO D. hustedtii & 14.2 & $12-3,110 / 13-1,60$ & $17-3,50 / 17-4,80$ \\
\hline LO D. maccollumii & 14.4 & $12-3,110 / 13-1,60$ & $16-1,50 / 16-2,50$ \\
\hline FO D. hyalina & - & & $18-3,54 / 18-4,57$ \\
\hline FO $A$. ingens var. nodus & - & & $18-4,57 / 18-5,54$ \\
\hline FO N. grossepunctata & 15.3 & & $19-3,53 / 20-1,53$ \\
\hline LO N. maleinterpretaria & 15.6 & $14-2,82 / 15-2,82$ & $20-4,50 / 20-5,50$ \\
\hline FO D. lauta & 16.0 & $14-2,82 / 15-2,82$ & $20-5,50 / 20-6,50$ \\
\hline FO D. maccollumii & 17.2 & $16-2,77 / 17-2,77$ & $20-6,50 / 21-1,50$ \\
\hline FO C. nicobarica & 17.8 & $18-3,77 / 18-4,77$ & $20-6,50 / 21-1,50$ \\
\hline LO T. spumellaroides & $20.1-20.3$ & $21-6,60 / 22-1,60$ & $22-2,50 / 22-3,50$ \\
\hline FO T. fraga & $20.8-20.9$ & $21-6,60 / 22-1,60$ & \\
\hline FO N. maleinterpretaria & 22.4 & $22-1,60 / 22-2,60$ & $29-2,62 / 29-5,4$ \\
\hline FO $T$. spumellaroides & $21.8-22.1$ & & $27-4,50 / 27-5,50$ \\
\hline FO $R$. schraderi & $27.1-27.2$ & & $31-2,139 / 31-3,139$ \\
\hline FO $R$. gelida & $27.5-27.7$ & & $31-2,139 / 31-3,139$ \\
\hline
\end{tabular}

Note: $\mathrm{FO}=$ first occurrence, $\mathrm{LCO}=$ last common occurrence, $\mathrm{LO}=$ last occurrence,

$\mathrm{FCO}=$ first common occurrence.

Nitzschia pseudokerguelensis Schrader, 1976, pl. 15, figs. 13-15.

Nitzschia reinholdii Kanaya ex Barron et Baldauf; Schrader, 1973, pl. 4, figs.

$12-16$, pl. 5, figs. $1-9$.

Nitzschia ritscheri (Hustedt) Hasle; Fenner et al., 1976, pl. 3, figs. 1-12.

Nitzschia rolandii Schrader emend. Koizumi; Akiba, 1985, pl. 25, figs. 1-6.

Nitzschia separanda (Hustedt) Hasle; Fenner et al., 1976, pl. 1, figs. 1-16, pl. 2, figs. 23-29.

Nitzschia weaveri Ciesielski, 1983, pl. 1, figs. 1-10. (Pl. 7, Fig. 5)

Nitzschia sp. 17 sensu Schrader, 1976, pl. 2, fig. 10; pl. 3, figs. 13-15.

Paralia clavigera (Grunow); Melosira clavigera Grunow; Harwood, 1986b, pl. 2, fig. 11.

Paralia sulcata Ehrenberg; Melosira sulcata (Ehrenberg) Kutzing; Schrader, 1973, pl. 20, fig. 9.

Pleurosigma sp. Specimens of Pleurosigma were not differentiated and are recorded as Pleurosigma sp.

Porosira glacialis (Grunow) Joergensen; Schrader and Fenner, 1976, pl. 16, figs. $1-4,13$, pl. 17 , fig. 1 .

Pseudorocella barbadensis Deflandre; Fenner, 1978, pl. 23, figs. 13-16, pl. 22 , figs. $1-3$. (not a diatom)

Pseudostictodiscus picus Hanna, Fenner, 1978, pl. 1, fig. 10.

Pseudotriceratium chenevieri (Meister) Gleser; Gombos and Ciesielski, 1983, pl. 17, fig. 4.

Pseudotriceratium radiosoreticulatum Grunow; Gombos and Ciesielski, 1983, pl. 17, fig. 1-3.

Pterotheca danica (Grunow) Forti, Gombos and Ciesielski, 1983, pl. 13, figs. $1-3,9$.

Pyxilla group. Specimens of Pyxilla were not differentiated and are recorded as Pyxilla group.

Raphidodiscus marylandicus Christian; Schrader, 1976, pl. 5, fig. 19; pl. 15, fig. 16.

Rhizosolenia antarctica Fenner; Fenner, 1985, pl. 11, fig. 12.

Rhizosolenia barboi (Brun) Tempère et Peragallo; Schrader, 1976, pl. 9, figs. 11-13.

Rhizosolenia hebetata f. hiemalis Gran; Schrader, 1976, pl. 9, figs. 1, 3 (not 2). (PI. 7, Fig. 7)

Rhizosolenia miocenica Schrader, 1973, pl. 25, figs. 1, 11.
Rhizosolenia oligocenica Schrader, 1976, pl. 9, fig. 7. Synonym: Rhizosolenia gravida Gombos and Ciesielski, 1983, pl. 11, figs. 1-7.

Rhizosolenia praebarboi Schrader, 1973, pl. 24, figs. 1-3.

Rhizosolenia styliformis Brightwell; Fenner et al., 1976, pl. 13, figs. 3-5, 9.

Rocella gelida (Mann) Bukry; Gombos and Ciesielski, 1983, pl. 6, figs. 1-6; pl. 26, fig. 1.

Rocella praenitida (Fenner) Fenner; Harwood, in press, pl. 5, fig. 4.

Rocella schraderi Bukry; Gombos and Ciesielski, 1983, pl. 22, fig. 6.

Roceila vigilans (Kolbe) Fenner; Gombos and Ciesielski, 1983, pl. 6, figs. 7-10; pl. 26, fig. 2.

Rossiella paleaea (Grunow) Desikachary et Maheshwari; Barron, 1985a, pl. 9, fig. 6. Synonym: Cussia paleacea (Grunow) Schrader.

Rossiella symmetrica Fenner; Fenner, 1985, pl. 7, figs. 5-7.

Rouxia antarctica Heiden and Kolbe; Schrader, 1976, pl. 5, figs. 1-8.

Rouxia californica Peragallo; Schrader, 1976, pl. 5, fig. 21. (Pl. 5, Fig. 6)

Rouxia diploneides Schrader, 1973, pl. 3, figs. 24, 25.

Rouxia fusiformis Barron; Barron, 1981, pl. 5, fig. 11.

Rouxia granda Schrader; Gombos and Ciesielski, 1983, pl. 21, fig. 11.

Rouxia heteropolara Gombos; Gersonde and Burckle, 1990, pl. 5, fig. 2.

Rouxia naviculoides Schrader, 1973, pl. 3, figs. 27-32.

Rouxia obesa Schrader in Schrader et Fenner, 1976, pl. 24, figs. 5, 6.

Rouxia peragalli Brun et Héribaud; McCollum, 1975, pl. 12, figs. 1, 2. (Pl. 5, Figs. 7,8)

Rutilaria sp.

Sceptroneis grunowii Anissimova; Schrader et Fenner, 1976, pl. 22, figs. 26-28; pl. 23, fig. 8; pl. 25, figs. 7, 9 .

Sceptroneis lingulatus Fenner, 1978, pl. 31, figs. 8-10.

Sceptroneis pesplanus Fenner et Schrader; Fenner, 1985, pl. 9, fig. 9.

Sceptroneis tenue Schrader et Fenner, 1976, pl. 3, figs. 1-4; pl. 24, figs. 11-13.

Stellarima group. Specimens of Stellarima were not differentiated; this includes specimens of $S$. microtrias (Ehrenberg) Hasle and Sims and $S$. stellaris (Roper) Hasle and Sims; Harwood, in press, pl. 1, figs. 3, 4.

Stephanogonia hanzawae Kanaya; Harwood, 1986b, pl. 4, fig. 10.

Stephanopyxis grunowii Grove et Sturt; Harwood, in press, pl. 2, figs. 5, 6.

Stephanopyxis hyalomarginata Hajòs, 1976, pl. 19, figs. 11, 12.

Stephanopyxis marginata Grunow; Baldauf and Monjanel, 1989, pl. 3, fig. 5. 
Stephanopyxis splendidus (Greville) Harwood, in press, pl. 2, figs. 1-4. Synonym: Thalassiosira hydra Gombos in Gombos and Ciesielski, 1983, pl. 7, figs. $1-6$.

Stephanopyxis superba (Greville) Grunow; Baldauf and Monjanel, 1989, pl. 2, fig. 4.

Stephanopyxis turris (Greville et Arnott) Ralfs; Schrader et Fenner, 1976, pl. 30 , figs. 1-10, 14; pl. 37, figs. 17-19.

Stephanopyxis turris var. cylindrus Grunow, Dzinoridze et al., 1978, pl. 1, fig. 8.

Synedra jouseana Sheshukova-Poretzkaya; Schrader, 1973, pl. 23, figs. 21-23, $25,38$.

Thalassionema nitzschioides Grunow; Fenner et al., 1976, pl. 14, figs. 11.

Thalassionema nitzschioides var. parva Heiden; Fenner et al., 1976, pl. 14, fig. 10.

Thalassiosira bukryi Barron; Barron, 1985a, pl. 11, fig. 3.

Thalassiosira burckliana Schrader; Barron, 1985a, pl. 11, fig. I. (Pl. 6, Fig. 8)

Thalassiosira convexa Mukhina; Barron, 1985a, pl. 11, fig. 13.

Thalassiosira convexa var. aspinosa Schrader; Barron, 1985a, pl. 11, figs. 8, 12.

Thalassiosira eccentrica (Ehrenberg) Cleve; Fenner et al., 1976, pl. 10, figs.

$$
1,2,4,5 \text {. }
$$

Thalassiosira fraga Schrader in Schrader et Fenner, 1976, pl. 16, figs. 9-12.

Thalassiosira gersonde $i$ Barron n. sp., Barron and Baldauf, herein (see preceding).

Thalassiosira gravida Cleve, Akiba and Yanagisawa, 1985, pl. 10, figs. 1-4.

Thalassiosira grunowii Akiba et Yanagisawa, 1985, pl. 27, fig. 5; pl. 29, figs. 1-8b; pl. 30, figs. 1-10. Synonym: Coscinodiscus plicatus Grunow.

Thalassiosira hydra Gombos in Gombos and Ciesielski, 1983, pl. 7, figs. 1-6. Harwood (1989) suggested that specimens of these are resting spores of Stephanopyxis splendidus (Greville) Harwood.

Thalassiosira inura Gersonde in Gersonde and Burckle, 1990, pl. 3, figs. 15-17; pl. 5, fig. 14. T. inura is not separated from $T$. gracilis in this manuscript. (Pl. 6, Fig. 9)

Thalassiosira jacksonii Koizumi et Barron; Akiba, 1985, pl. 11, fig. 2. (PI. 6, Fig. 7)

Thalassiosira kolbei (Jousé) Gersonde; Gersonde and Burckle, 1990, pl. 3, fig. 1. Synonym: Coscinodiscus kolbei Jousé.

Thalassiosira lentiginosa (Janisch) Fryxell. Synonym: Coscinodiscus lentiginosus Janisch; Fenner et al., 1976, pl. 7, figs. 4-6.
Thalassiosira lentiginosa var. obovatus (Castracane) Fryxell. Synonym: Coscinodiscus lentiginosus var. obovatus (Castracane) Ciesielski, 1983, pl. 4, figs. 4-8.

Thalassiosira mahoodii Barron n. sp., Baldauf and Barron, herein (see preceding).

Thalassiosira miocenica Schrader; Barron, 1985a, pl. 11, fig. 11. (PI. 6, Fig. 2)

Thalassiosira nativa Sheshukova-Poretzkaya sensu Schrader, 1973, pl. 11, figs. 23, 24. (P1. 6, Fig. 5)

Thalassiosira oestrupii (Ostenfeld) Proschkina-Lavrenko; Schrader, 1973, pl. 11 , figs. 16-22, 26-33, 36, 39-45.

Thalassiosira oliverana (O'Meara) Sournia. Synonym: Schimperiella antarctica Karsten; Fenner et al., 1976, pl. 14, figs. 1-5.

Thalassiosira praeconvexa Burckle; Barron, 1985a, pl. 11, fig. 7.

Thalassiosira praeoestrupii Dumont et al., 1986, pl. 1, figs. 19, 12.

Thalassiosira primalabiata Gombos in Gombos and Ciesielski, 1983, pl. 9, figs. 1-8.

Thalassiosira spinosa Schrader, 1976, pl. 6, figs. 5-7.

Thalassiosira spumellaroides Schrader, 1976, pl. 6, figs. 1-2.

Thalassiosira symbolophora Schrader, 1974, pl. 4, figs. 1-8.

Thalassiosira torokina Brady; Harwood, 1986a, pl. 15, figs. 11, 13, 14; pl. 25, figs. 1-3; Ciesielski, 1983, pl. 7, figs. 3-6. (Pl. 6, Figs. 1, 4)

Thalassiosira yabei (Kanaya) Akiba et Yanagisawa, 1985, pl. 27, figs. 1-2; pl. 28, figs. 1-9. Synonym: Coscinodiscus yabei Schrader.

Thalassiosira sp. 1 sensu Barron, 1980; pl. 5, figs. 6, 7; Barron, 1981, pl. 5, fig. 5; Thalassiosira sp. cf. T. praeconvexa Burckle sensu Akiba, 1985, pl. 8, fig. 5. This small, nondistinct Thalassiosira typical of the early late Miocene of both the middle- to high-latitude North Pacific and the Southern Ocean is not described here because of the lack of SEM illustrations. Synonym: T. praeconvexa Burckle sensu Schrader, 1973, pl. 11, figs. 10/15. (PI. 1, Figs. 3, 5, 6)

Thalassiothrix longissima Cleve et Grunow; Schrader, 1976, pl. 1, figs. 5, 6, 17.

Triceratium condecorum Brightwell; Fenner, 1978, pl. 28, fig. 7; pl. 29, fig. 1. Triceratium macroporum Hajòs; Gombos and Ciesielski, 1983, pl. 17, fig. 6 . Triceratium unguiculatum Greville; Gombos, 1976, pl. 33, figs. 1, 3; pl. 34, figs. $1-6$.

Trinacria excavata Heiberg; Schrader, 1976, pl. 14, fig. 15. 

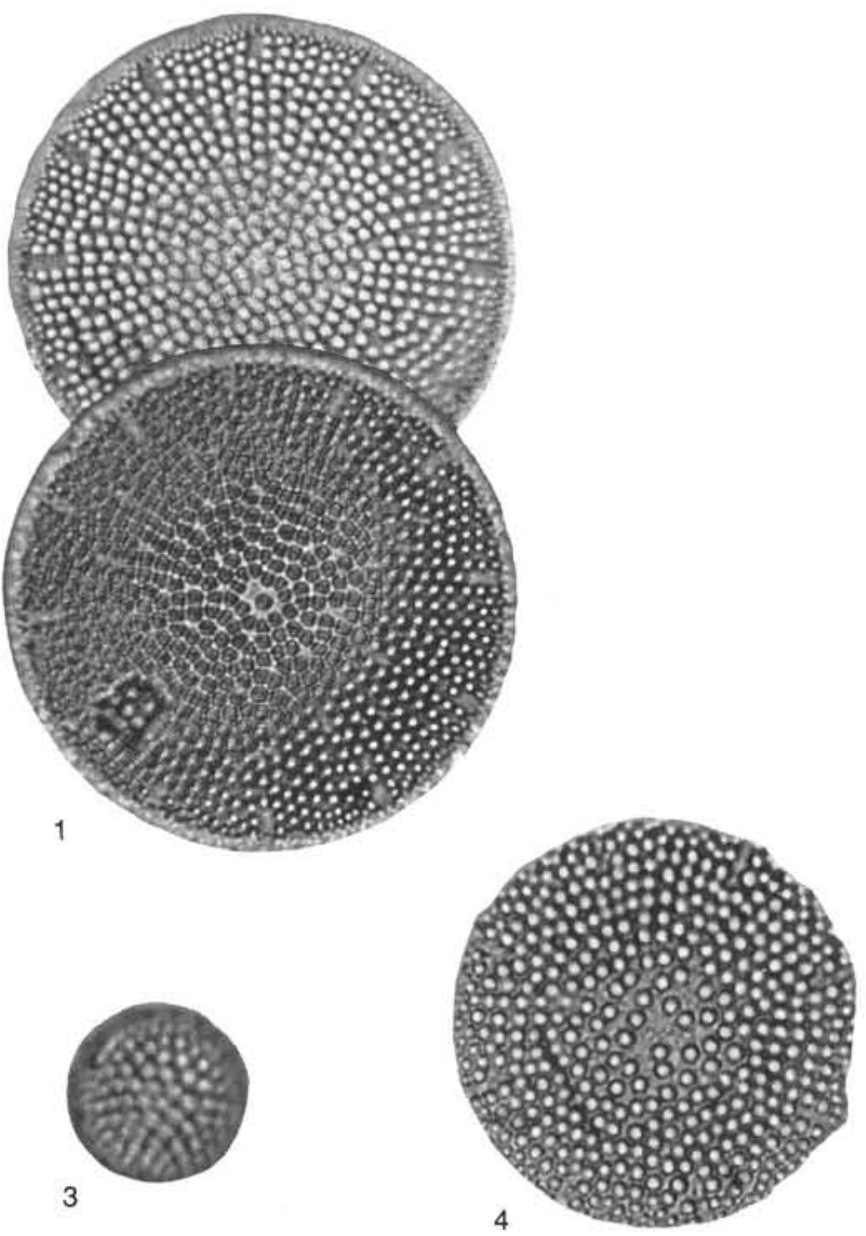

3
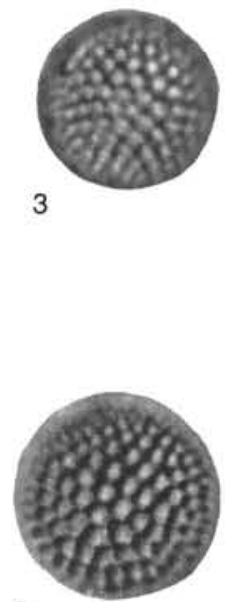

5

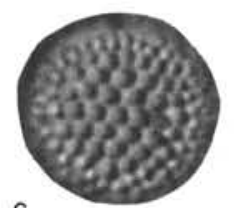

6

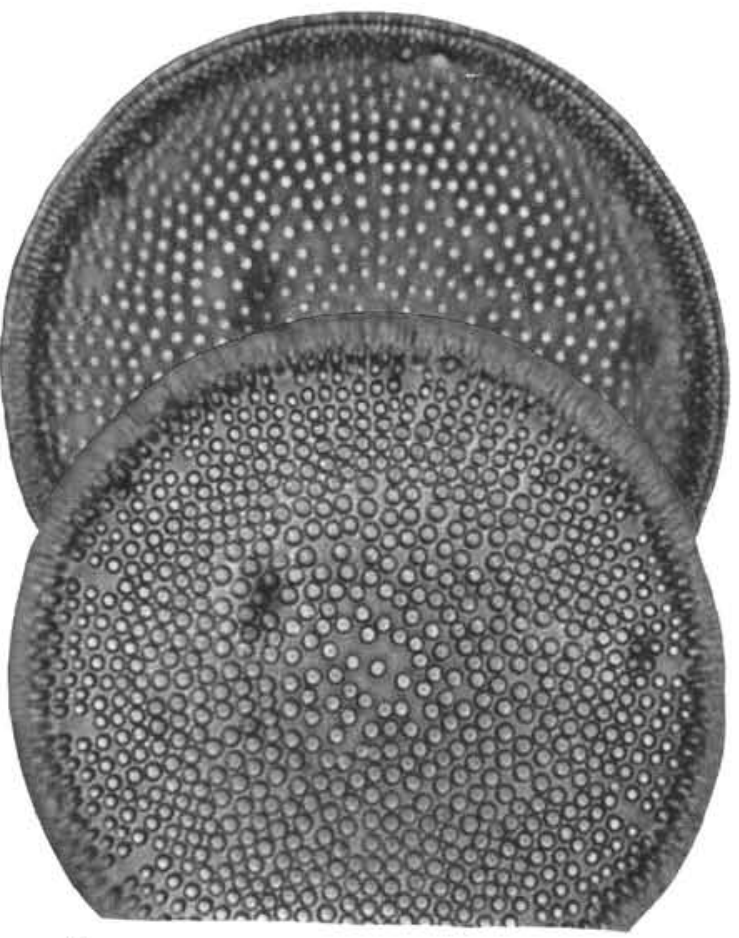

2

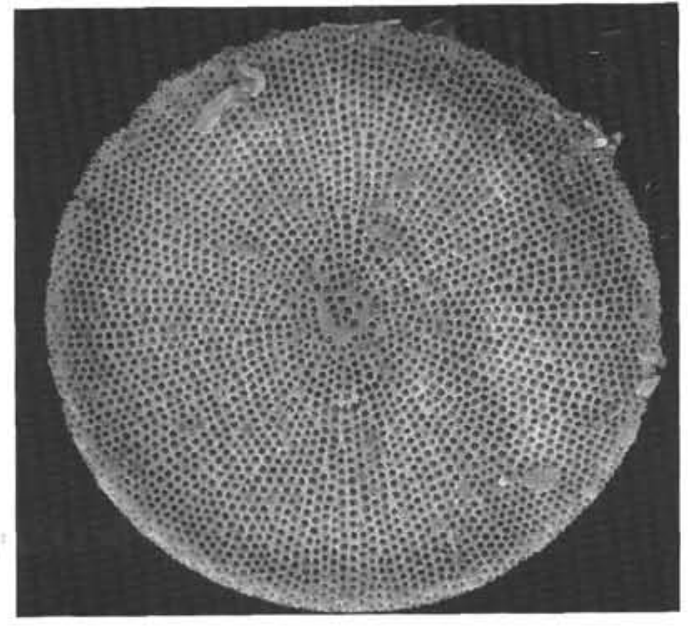

7

$40 \mu \mathrm{m}$

Plate 1. 1, 2, 4. Actinocyclus fryxellae Barron n. sp. (1) Sample 119-744D-5H1,34 cm, low and high focus; (2) holotype, USNM 444983, Sample 119-746A-7H-6, $60-62 \mathrm{~cm}$, diameter $49 \mu \mathrm{m}$, low and high focus, note pseudonodulus immediately left of 12 o'clock; (4) Sample 119-744B-5H-1, 60-62 cm, diameter 27 $\mu \mathrm{m}$. 3, 5, 6. Thalassiosira sp. 1 sensu Barron 1980, Sample 119-744B4H-7, 60-62 cm. Diameters: (3) $9 \mu \mathrm{m}$, (5) $11 \mu \mathrm{m}$, (6) $10 \mu \mathrm{m}$. 7. Actinocyclus sp. cf. Actinocyclus tsugaruensis Kanaya, Sample 119-746A-7H-6, 60-62 cm, SEM photograph, note pseudonodulus at 3 o'clock, scale bar represents $40 \mu \mathrm{m}$. 

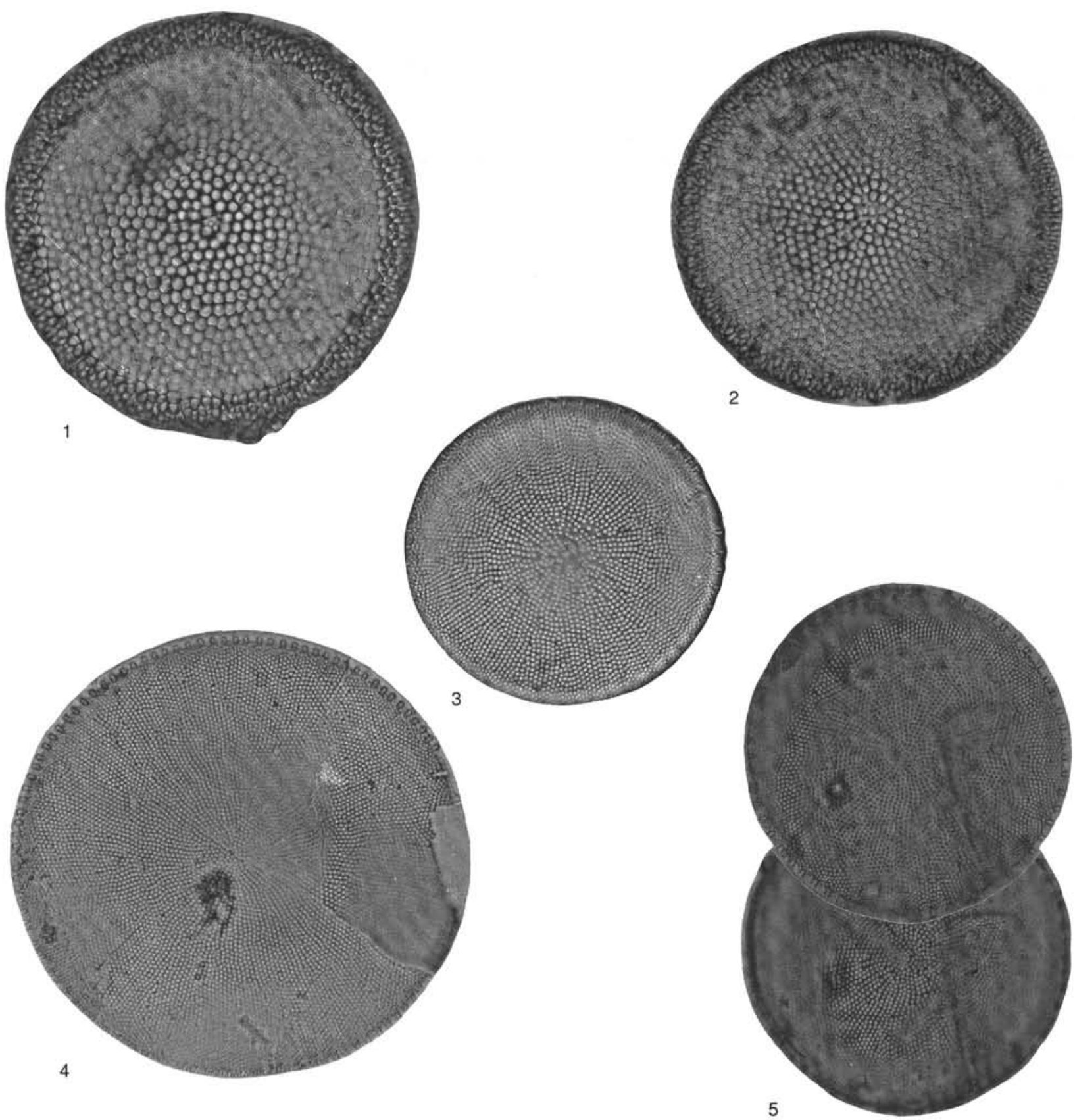

Plate 2. 1, 2. Thalassiosira gersondei Barron n. sp., Sample 119-746A-7H-6, 60-61 cm. (1) Holotype, USNM 444984, diameter 55 $\mu \mathrm{m}$; (2) isotype, USNM 444985, diameter $50 \mu \mathrm{m}$. 3-5. Thalassiosira mahoodii Barron n. sp., Sample 119-746A-7H-6, 60-62 cm. (3) Isotype, USNM 444987, diameter 46 $4 \mathrm{~m}$; (4) holotype, USNM 444986, diameter $75 \mu \mathrm{m}$; (5) isotype, USNM 444988, diameter $49 \mu \mathrm{m}$. 


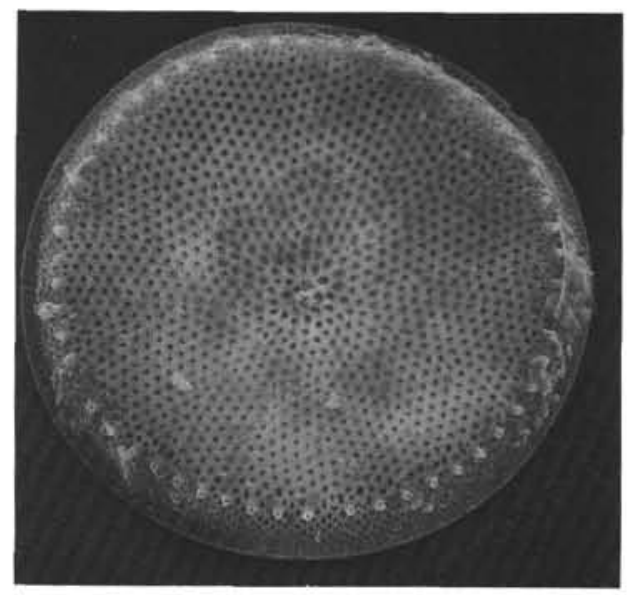

1

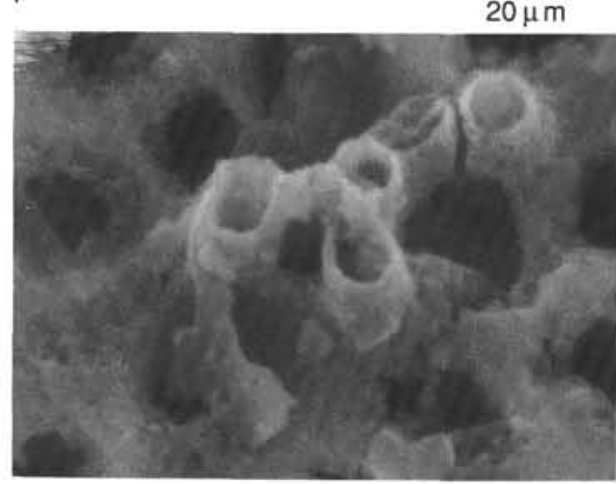

3

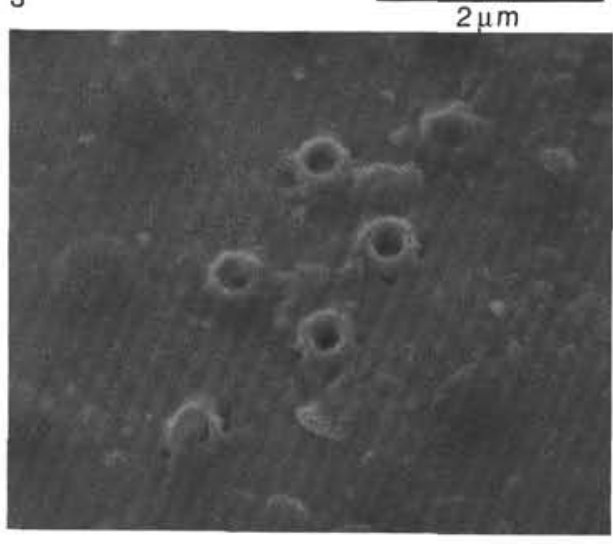

5

$2 \mu \mathrm{m}$

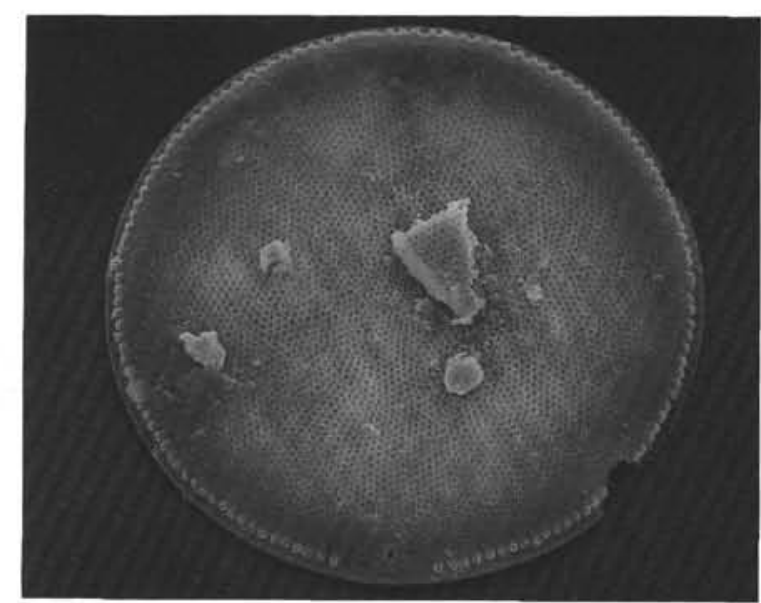

2

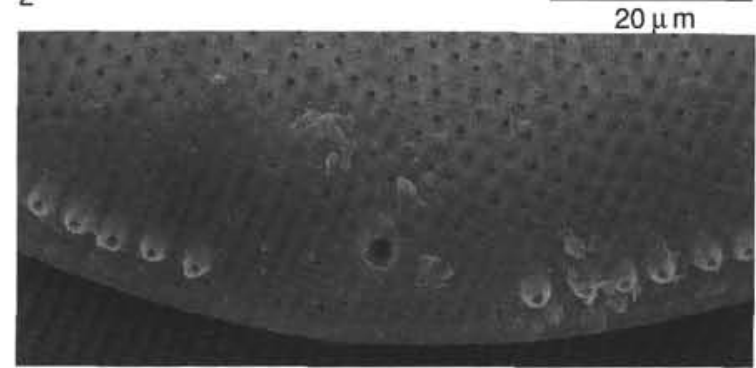

4

$4 \mu \mathrm{m}$

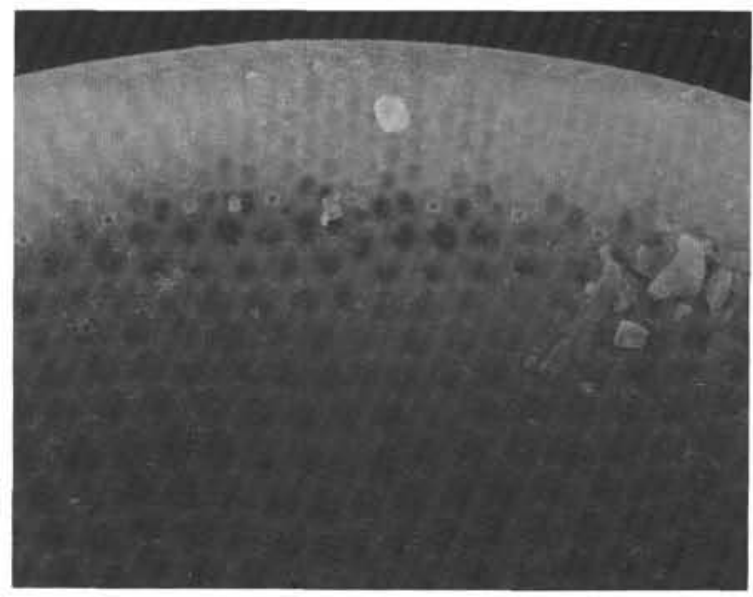

6

$4 \mu \mathrm{m}$

Plate 3. SEM photographs of diatoms from Sample 119-746A-7H-6, 60-62 cm. 1, 3, 5, 6. Thalassiosira gersondei Barron n. $\mathrm{sp} .(1) \mathrm{Valve}$ view, scale bar $=20$ $\mu \mathrm{m} ;(3)$ close-up view of the center of Figure 1 showing the central strutted processes arranged in a trifulate pattern, scale bar $=2 \mu \mathrm{m} ;(5)$ internal view of the openings at base of the central strutted processes, scale bar $=2 \mu \mathrm{m} ;(6)$ internal view of the margin showing internal openings of marginal processes, scale bar $=$ $4 \mu \mathrm{m} .2,4$. Thalassiosira mahoodii Barron n. sp. (2) Valve view, scale bar $=20 \mu \mathrm{m}$; (4) close-up view of the 6 o'clock region of the specimen in Figure 2 showing the interruption of the marginal processes at the external opening of the labiate process, scale $b a r=4 \mu \mathrm{m}$. 


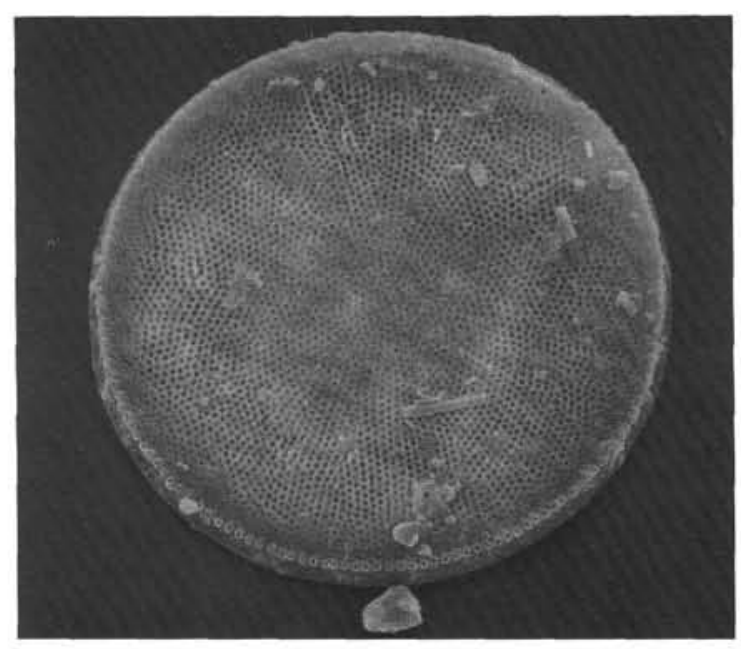

1

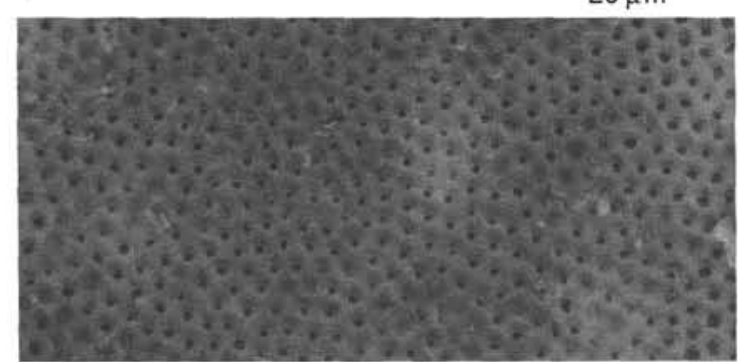

5
3

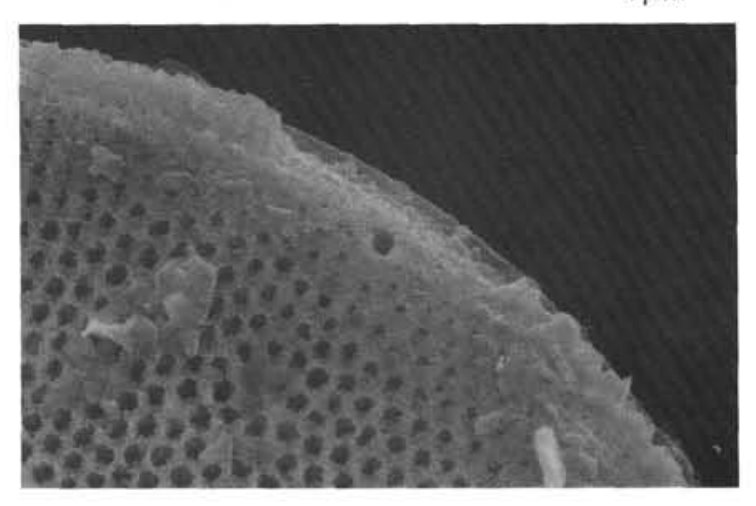

$4 \mu \mathrm{m}$

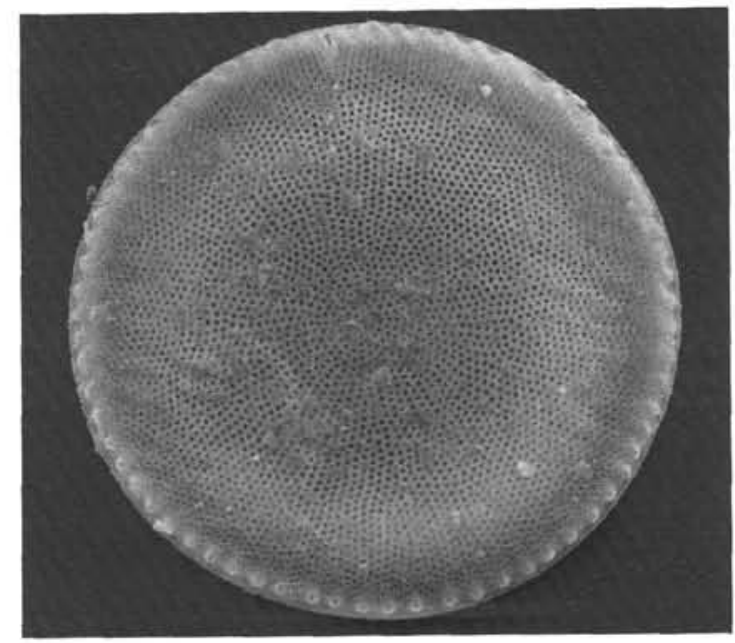

2

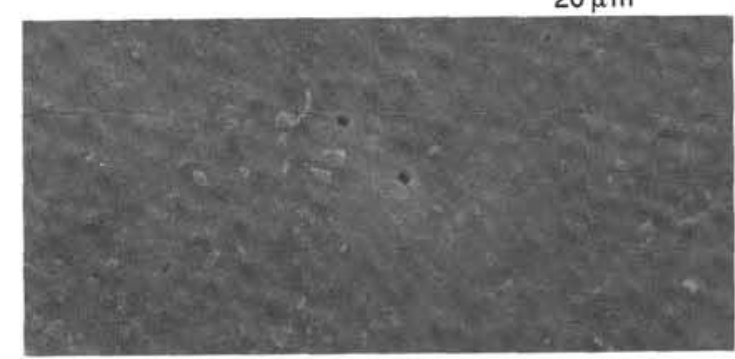

4

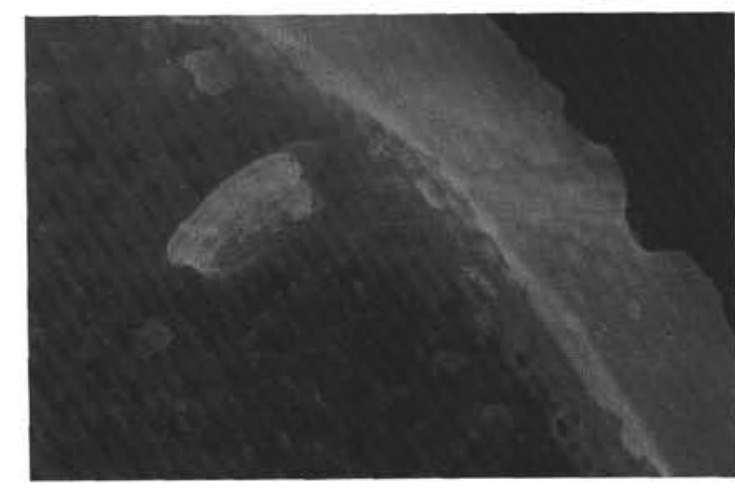

6

$4 \mu \mathrm{m}$

Plate 4. SEM photographs of Thalassiosira mahoodii Barron n. sp, Sample 119-746A-7H-6, 60-62 cm. 1, 2. Valve views, scale bar $=20 \mu \mathrm{m}$. 3. Close-up view of the center of the specimen in Figure 1, scale bar $=4 \mu \mathrm{m}$. 4. Internal view of the valve center showing two pores that may be the remains of central processes, scale $\mathrm{bar}=4 \mu \mathrm{m}$. 5. Close-up view of the external opening of the labiate process, 1 o'clock position of Figure 1. 6. Labiate process in the interior of the valve near the margin. 

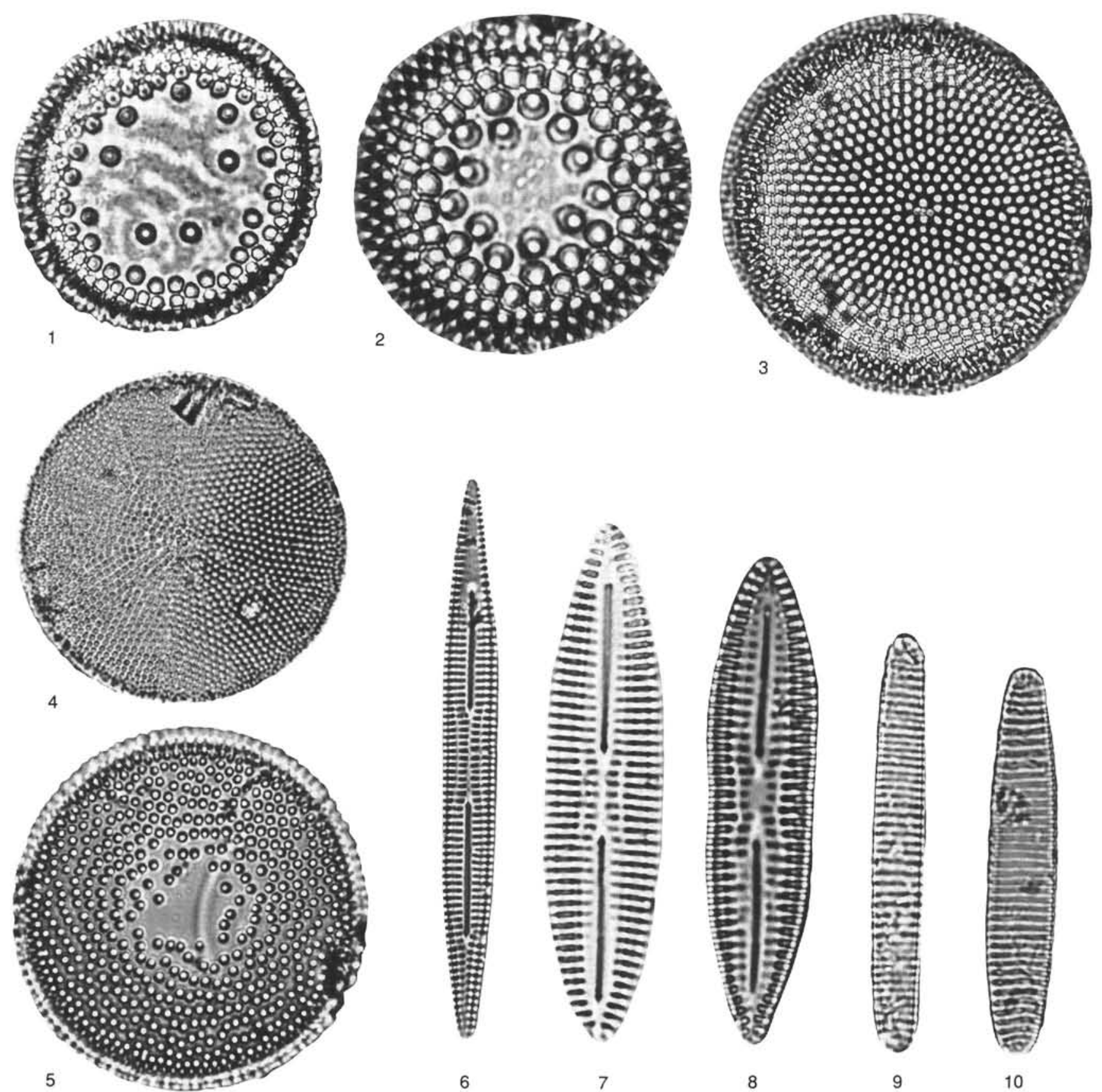

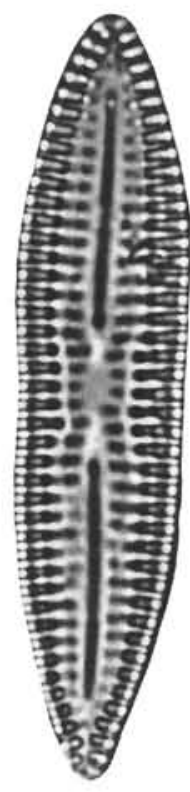

8

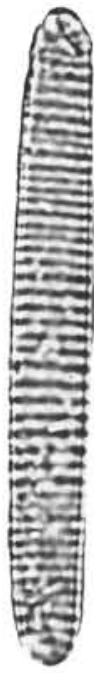

9

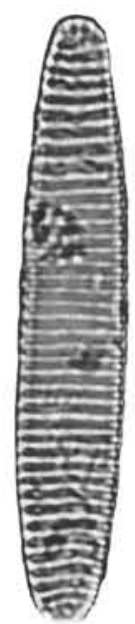

10

Plate 5. 1. Actinocyclus ingens var. ovalis Gersonde, diameter $31 \mathrm{~mm}$, Sample 119-746A-8H-1, 60-62 cm. 2. Actinocyclus ingens Rattray, diameter $25 \mathrm{~mm}$, Sample 119-737B-8R-2, 57-59 cm. 3. Thalassiosira sp. cf. Thalassiosira mahoodii Barron n. sp., diameter $35 \mathrm{~mm}$, Sample 119-746A-8H-1, 60-62 cm. 4. Thalassiosira gersondei Barron n. sp., diameter $42 \mathrm{~mm}$, Sample 119-746A-11H-5, 60-62 cm. 5. Actinocyclus sp. cf. A. ochotensis Jousé, diameter $34 \mathrm{~mm}$, Sample 119-737A-13H-2, 60-62 cm. 6. Rouxia californica Peragallo, length $72 \mathrm{~mm}$, Sample 119-737A-23X-2, 57-59 cm. 7, 8. Rouxia peragalli Brun et Héribaud variety. (7) Length $40 \mathrm{~mm}$, Sample 119-737A-13H-2, 57-59 cm; (8) length $38 \mathrm{~mm}$, Sample 119-737A-16H-2, 57-59 cm. 9. Nitzschia praecylindrus Gersonde, length 32 $\mathrm{mm}$, Sample 119-746A-8H-CC. 10. Nitzschia januaria Schrader, length $32 \mathrm{~mm}$, Sample 119-746A-4H-6, 60-62 cm. 

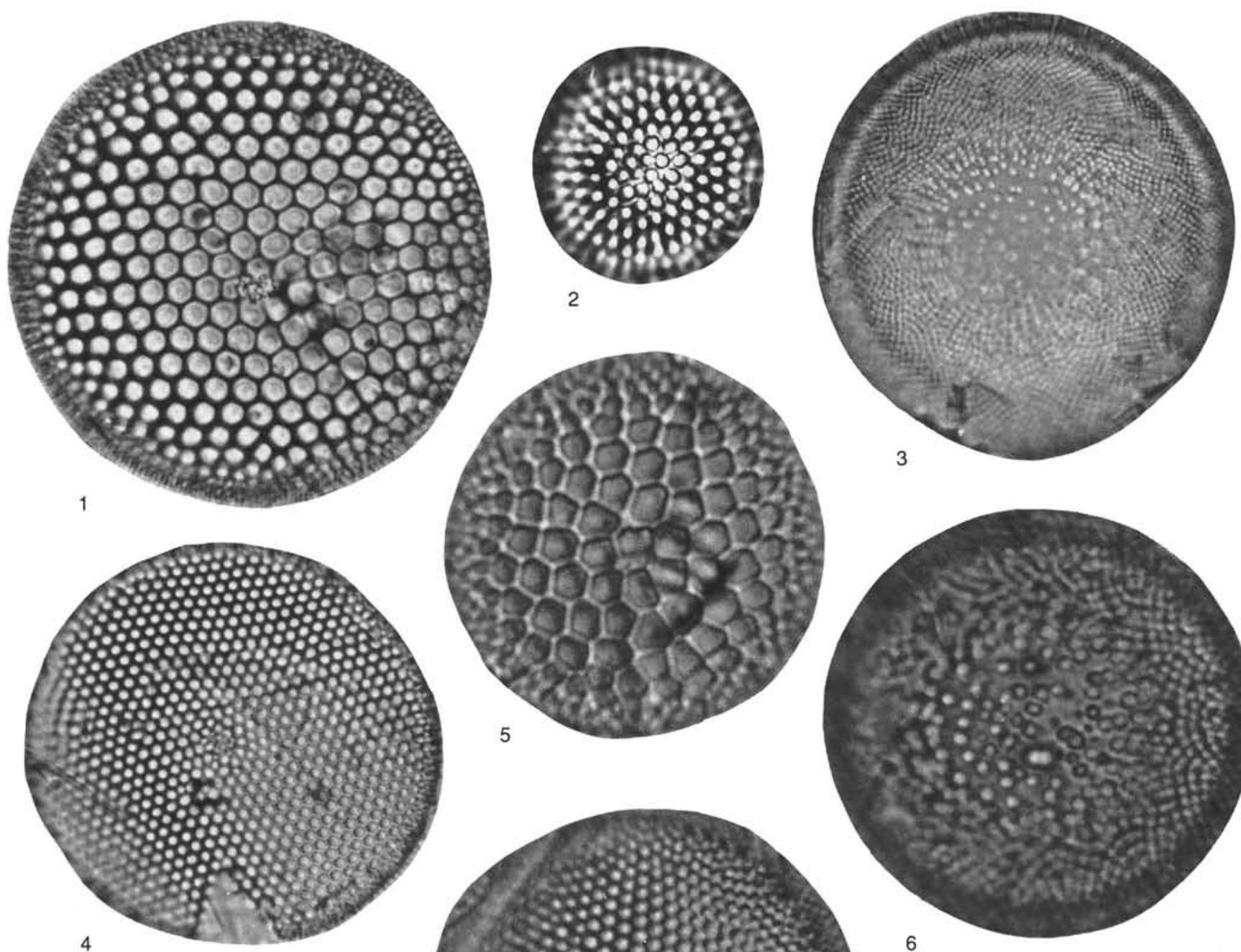

2
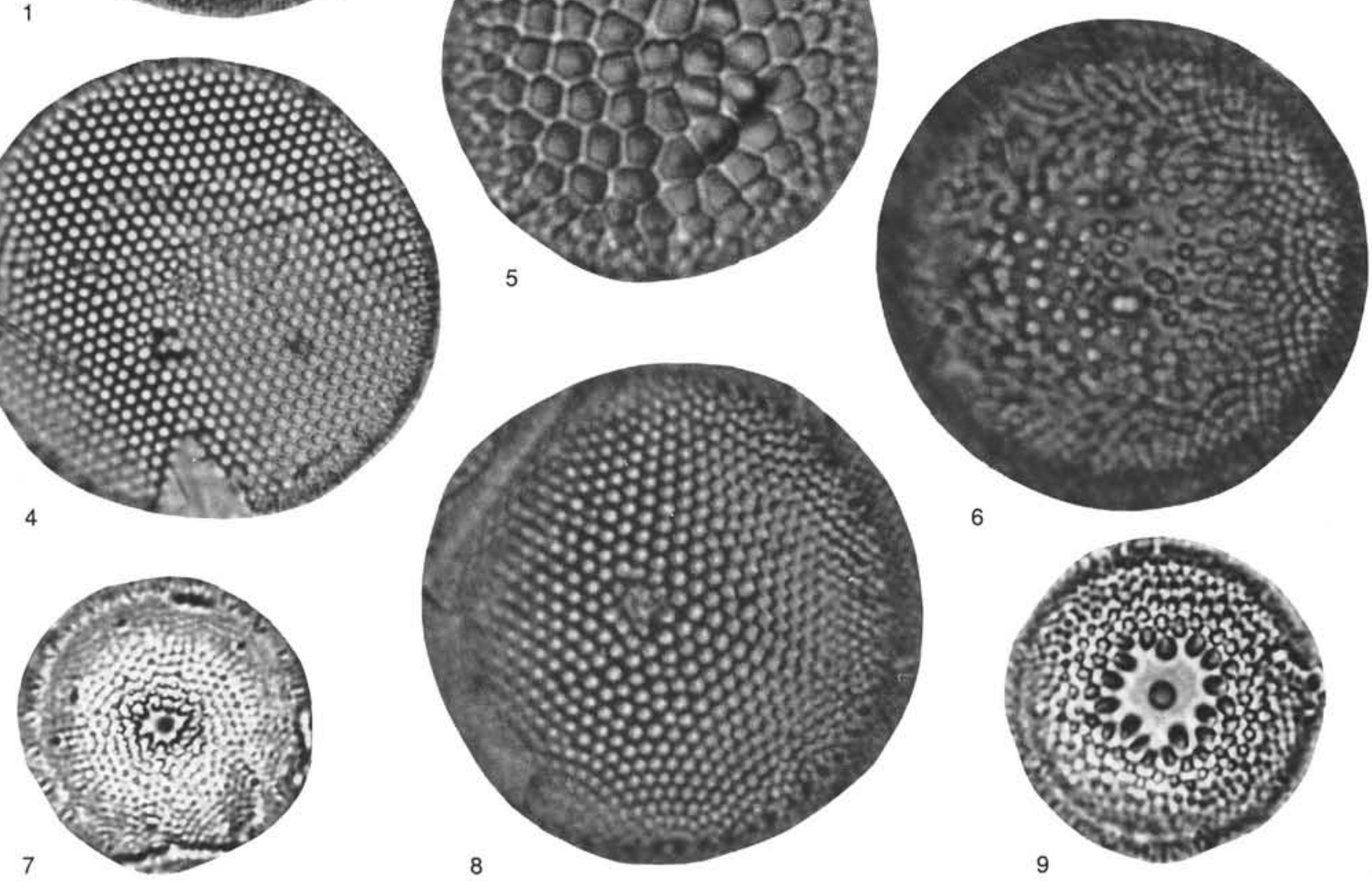

Plate 6. 1. Thalassiosira torokina Brady, diameter $53 \mu \mathrm{m}$, Sample $119-737 \mathrm{~A} 26 \mathrm{X}-1,57-59 \mathrm{~cm}$. 2. Thalassiosira miocenica Schrader, diameter $16 \mu \mathrm{m}$. 3, 6 . Cosmiodiscus intersectus (Brun) Jousé. (3) Sample 119-737A-26X-1, 57-59 cm, diameter $51 \mu \mathrm{m}$; (6) Sample 119-737A-27X-1, 57-59 cm, diameter $25 \mu \mathrm{m} .4$. Thalassiosira torokina Brady, diameter $25 \mu \mathrm{m}$, Sample 119-737A-27X-1, 57-59 cm. 5. Thalassiosira nativa Sheshukova sensu Schrader, 1973 , diameter $17 \mu \mathrm{m}$, Sample 119-737A-26X-1, 57-59 cm. 7. Thalassiosira jacksonii Koizumi et Barron, diameter $16.5 \mu \mathrm{m}$, Sample 119-737A-9H-5, 57-59 cm. 8. Thalassiosira burckliana Schrader, diameter $27 \mu \mathrm{m}$, Sample 119-737A-26X-1, 57-59 cm. 9. Thalassiosira inura Gersonde, diameter 16 $\mu \mathrm{m}$, Sample 119-737A-8H-6, 57-59 $\mathrm{cm}$. 

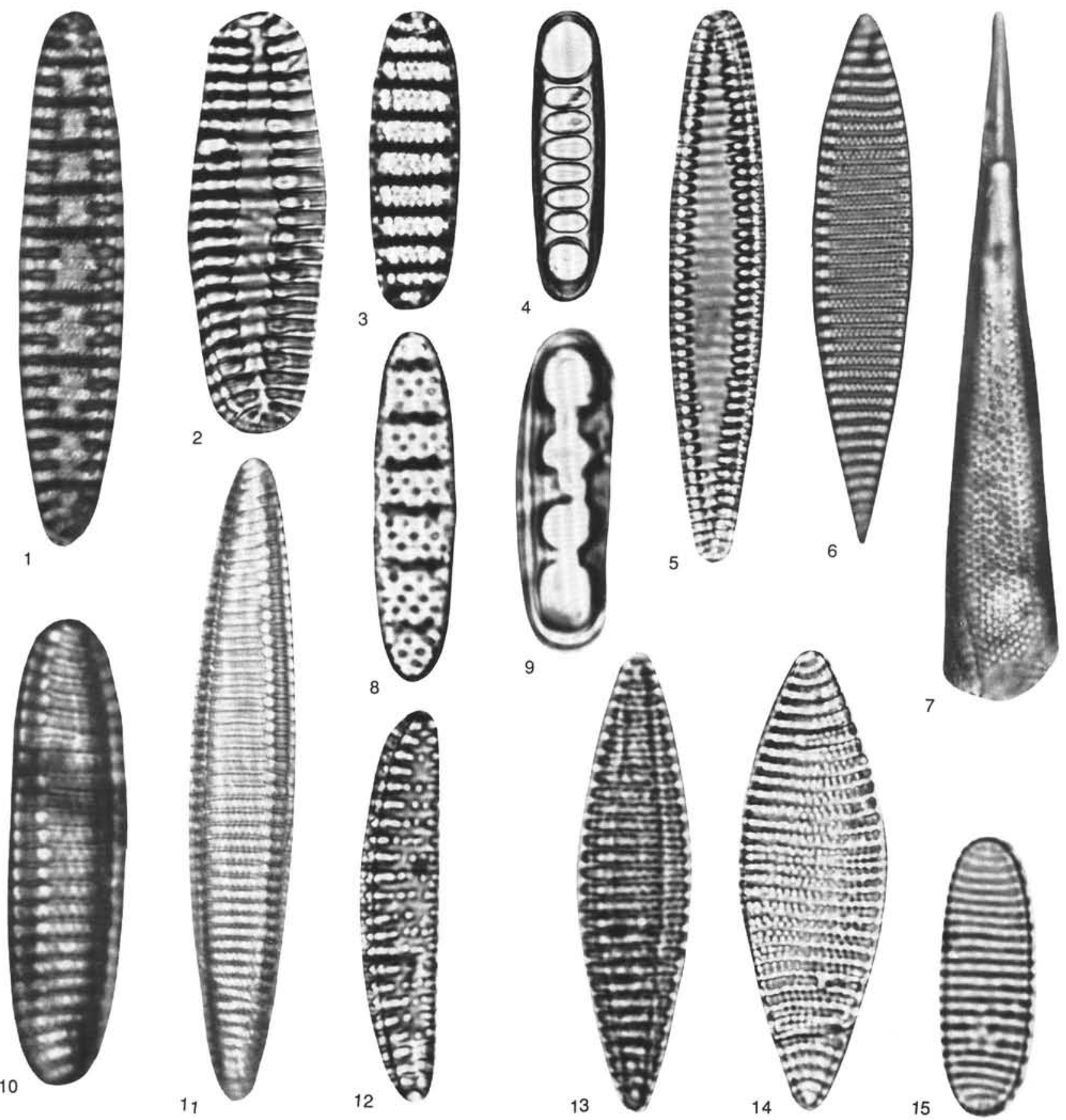

15

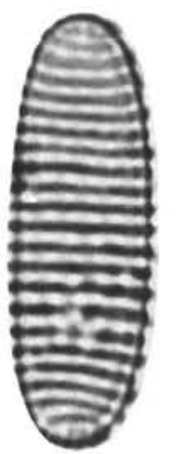

Plate 7. 1. Denticulopsis hustedtii (Simonsen et Kanaya) Simonsen, length $48 \mu \mathrm{m}$, Sample 119-737A-27X-3, 57-59 cm. 2. Nitzschia denticuloides Schrader, length $31 \mu \mathrm{m}$, Sample 119-737D-7R-2,57-59 cm. 3. Denticulopsis lauta (Bailey) Simonsen et Kanaya, length $23 \mu \mathrm{m}$, Sample 119-737B-6R-4, 57-59 cm. 4. Denticulopsis dimorpha (Schrader) Simonsen, length $32 \mu \mathrm{m}$, Sample 119-737B-7R-1, 62-64 cm. 5. Nitzschia weaveri Ciesielski, length 46 $\mu \mathrm{m}$, Sample 119-737A-7H-4, 57-59 $\mathrm{cm}$. 6. Nitzschia donahuensis Schrader, length $50 \mu \mathrm{m}$, Sample 119-737A-27X-3, 57-59 cm. 7. Rhizosolenia hebetata f. hiemalis Gran, length $58 \mu \mathrm{m}$, Sample 119-737A-26X-1, 57-59 cm. 8. Crucidenticula nicobarica (Grunow) Akiba and Yanagisawa, length $22 \mu \mathrm{m}$, Sample 119-737B-9R-2, 56-58 cm. 9. Denticulopsis praedimorpha Akiba, length $20.5 \mu \mathrm{m}$, Sample 119-737B-8R-2, 57-59 cm. 10. Nitzschia cylindrica Burckle, length $28.5 \mu \mathrm{m}$, Sample $119-737 \mathrm{~A}-27 \mathrm{X}-1,57-59 \mathrm{~cm}$. 11. Nitzschia marina Grunow, length $60 \mu \mathrm{m}$, Sample 119-737A-27X-1, 57-59 cm. 12. Nitzschia praeinterfrigidaria McCollum, length $27 \mu \mathrm{m}$, Sample $119-737 \mathrm{~A}-7 \mathrm{H}-5,57-59 \mathrm{~cm}$. 13. Nitzschia miocenica Burckle, length $31 \mu \mathrm{m}$, Sample $119-746 \mathrm{~A}-4 \mathrm{H}-4,60-62 \mathrm{~cm}$. 14. Nitzschia barronii Gersonde, length $26 \mu \mathrm{m}$, Sample 119-737A7H-CC. 15. Nitzschia praecurta Gersonde, length $17 \mu \mathrm{m}$, Sample 119-746A-4H-2, 60-62 cm. 